\title{
The USGS Reference Sample Devonian Ohio Shale SDO-1
}

\section{U.S. GEOLOGICAL SURVEY BULLETIN 2046}

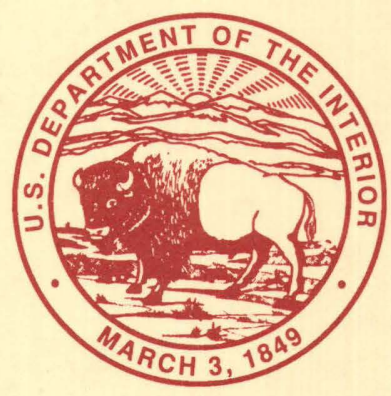




\section{AVAILABILITY OF BOOKS AND MAPS OF THE U.S. GEOLOGICAL SURVEY}

Instructions on ordering publications of the U.S. Geological Survey, along with prices of the last offerings, are given in the current-year issues of the monthly catalog "New Publications of the U.S. Geological Survey." Prices of available U.S. Geological Survey publications released prior to the current year are listed in the most recent annual "Price and Availability List." Publications that may be listed in various U.S. Geological Survey catalogs (see back inside cover) but not listed in the most recent annual "Price and Availability List" may be no longer available.

Prices of reports released to the open files are given in the listing "U.S. Geological Survey Open-File Reports," updated monthly, which is for sale in microfiche from U.S. Geological Survey ESIC-Open-File Report Sales, Box 25286, Denver, CO 80225. Reports released through the NTIS may be obtained by writing to the National Technical Information Service, U.S. Department of Commerce, Springfield, VA 22161; please include NTIS report number with inquiry.

Order U.S. Geological Survey publications by mail or over the counter from the offices given below.

\section{BY MAIL}

\section{Books}

Professional Papers, Bulletins, Water-Supply Papers, Techniques of Water-Resources Investigations, Circulars, publications of general interest (such as leaflets, pamphlets, booklets), single copies of Earthquakes \& Volcanoes, Preliminary Determination of Epicenters, and some miscellaneous reports, including some of the foregoing series that have gone out of print at the Superintendent of Documents, are obtainable by mail from

\section{U.S. Geological Survey, Map Distribution Box 25286, Bldg. 810, Federal Center Denver, CO 80225}

Subscriptions to periodicals (Earthquakes \& Volcanoes and Preliminary Determination of Epicenters) can be obtained ONLY from the

\section{Superintendent of Documents \\ Government Printing Office \\ Washington, D.C. 20402}

(Check or money order must be payable to Superintendent of Documents.)

\section{Maps}

For maps, address mail orders to

$$
\begin{gathered}
\text { U.S. Geological Survey, Map Distribution } \\
\text { Box 25286, Bldg. 810, Federal Center } \\
\text { Denver, CO } 80225
\end{gathered}
$$

Residents of Alaska may order maps from

\author{
U.S. Geological Survey, Earth Science Information Center \\ 101 Twelfth Ave. - Box 12 \\ Fairbanks, AK 99701
}

\section{OVER THE COUNTER}

\section{Books and Maps}

Books and maps of the U.S. Geological Survey are available over the counter at the following U.S. Geological Survey offices, all of which are authorized agents of the Superintendent of Documents:

- ANCHORAGE, Alaska-Rm. 101, 4230 University Dr.

- LAKEWOOD, Colorado-Federal Center, Bldg. 810

- MENLO PARK, California-Bldg. 3, Rm. 3128, 345 Middlefield Rd.

- RESTON, Virginia-USGS National Center, Rm. 1C402, 12201 Sunrise Valley Dr.

- SALT LAKE CITY, Utah-Federal Bldg., Rm. 8105, 125 South State St.

- SPOKANE, Washington-U.S. Post Office Bldg., Rm. 135, West 904 Riverside Ave.

- WASHINGTON, D.C.-Main Interior Bldg., Rm. 2650, 18th and C Sts., NW.

\section{Maps Only}

Maps may be purchased over the counter at the following U.S. Geological Survey offices:

- FAIRBANKS, Alaska-New Federal Bldg., 101 Twelfth Ave.

- ROLLA, Missouri-1400 Independence Rd.

- STENNIS SPACE CENTER, Mississippi-Bldg. 3101 


\section{The USGS Reference Sample Devonian Ohio Shale SDO-1}

Edited by JEAN S. KANE

This publication presents a collection of detailed analytical procedures used by some of the laboratories whose data was used to derive recommended concentrations for SDO-1 as a geochemical reference sample, and outlines the statistical evaluation used in the derivation of those concentrations from the total data base. 


\title{
U.S. DEPARTMENT OF THE INTERIOR BRUCE BABBITT, Secretary
}

\author{
U.S. GEOLOGICAL SURVEY \\ Dallas L. Peck, Director
}

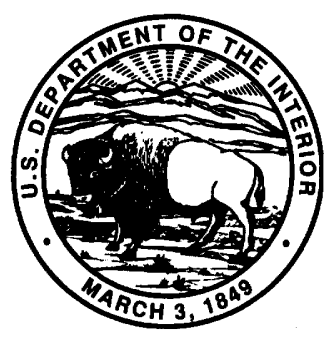

Any use of trade, product, or firm names in this publication is for descriptive purposes only and does not imply endorsement by the U.S. Government

For sale by

U.S. Geological Survey, Map Distribution

Box 25286, Bldg. 810, Federal Center

Denver, CO 80225

\section{Library of Congress Cataloging in Publication Data}

The USGS reference sample Devonian Ohio Shale SDO-1 / edited by Jean S.

Kane.

p. cm. - (U.S. Geological Survey bulletin ; 2046)

Includes bibliographical references.

Supt. of Docs. no.: 1 19.3:2046

1. Ohio Shale. I. Kane, Jean S. II. Series.

QE75.B9 no. 2046

[QE471.15.S5]

$557.3 \mathrm{~s}-\mathrm{dc} 20$

[553.2'83'0973] 


\section{CONTENTS}

[Letters designate the chapters]

A. Statistical Treatment of Contributed Data in the Derivation of Recommended Concentrations for Devonian Ohio Shale SDO-1, by Jean S. Kane

B. Determination of Major and 11 Trace Elements (XRF), Gold (AAS), Carbon and Sulfur (COUL) in USGS Devonian Ohio Shale SDO-1, by Otmar Spies, Bernhard Stribrny, Josef Konopasek, and Hans Urban

C. Inductively Coupled Plasma Determination of Nine Rare Earth Elements in the USGS Devonian Ohio Shale SDO-1, by Iwan Roelandts

D. Instrumental Neutron Activation Analysis of Devonian Ohio Shale SDO-1, by Gregory A. Wandless

E. Relation Between Volatile Components and Loss on Ignition, as Applied to the Analysis of USGS Reference Devonian Ohio Shale SDO-1 and Rare Earth Element Analyses, by Miroslav Huka and Ivan Rubeska

F. A Study to Determine Sources of Interlaboratory Variability in Measured Loss on Ignition (LOI) for Devonian Ohio Shale SDO-1, by Jean S. Kane and Carol J. Skeen

G. Analysis of SDO-1 Pressed Powder Pellets by X-ray Fluorescence-A Note of Caution, by Otto C. Kopp and Francis C. Furman 



\title{
The USGS Reference Sample Devonian Ohio Shale SDO-1
}

\author{
Edited by Jean S. Kane
}

\section{INTRODUCTION}

The United States Geological Survey (USGS) has maintained a geochemical reference sample (GRS) program since 1951, when the Fairbairn report on the cooperative analysis of G-1 and W-1 was issued. Many of the reference samples developed and issued to the worldwide geochemical community since then have been igneous rocks whose elemental concentrations are quite well established, making the GRS's comparable to National Institute of Standards and Technology (NIST) certified or standard reference samples (SRM's). For the most part, these igneous rock reference samples have trace element concentrations at rather low levels, approaching average crustal abundances. These materials are quite useful in baseline geochemical applications, but of less value in exploration programs or in studies of ore genesis where higher trace elemental abundances are characteristic of the samples actually being analyzed. These materials are sometimes poorly suited, by virtue of matrix mismatches, for use in studies of metamorphic and sedimentary rocks, including gas and oil shales and coal. USGS has begun developing a number of new reference samples to meet these added needs. This bulletin is devoted to the development of one of these new materials, Devonian Ohio Shale SDO-1. (Note that there is a homonym of this material, the marine sediment SDO-1 issued by the USSR Academy of Sciences (Arnautov, 1987).)

SDO-1, a gas shale, was first introduced in the seventies as a control sample during the Eastern Gas Shale Project sponsored by the U.S. Department of Energy (DOE) (1978), in which the USGS was a participant. The sample is a brownish-black, relatively radioactive shale (40-100 counts/second, or 2-4 times background over 0-200 ft) that weathers blocky and massive in the lower two-thirds of the unit and is less resistant in the upper one-third of the unit.

Manuscript approved for publication September 15, 1992.
Thin-sections show the shale to be organic-rich with a fine-ground clay matrix and abundant microfossils and pyrite; the pyrite is also observed in hand specimens (Kepferle and others, 1985; Provo and others, 1978). The sample was collected from a fresh road cut along U.S. Highway 64, about $8 \mathrm{mi}$ west of Morehead, Rowan County, $\mathrm{Ky}$, , at lat $38^{\circ} 09^{\prime} 56^{\prime \prime} \mathrm{N}$., long $83^{\circ} 35^{\prime} 38^{\prime \prime} \mathrm{W}$. (Kepferle and others, 1985).

A preliminary report (Kepferle and others, 1985) proposed elemental concentrations based on a limited data base. Recommended concentrations published since then (Kane and others, 1990) (table 1) were derived from a larger data set that included the original Eastern Gas Shale Project data, the International Geological Congress (IGC) Metalliferous Black Shales (\#254) Project, and concurrent contributions from USGS and other laboratories.

This report contains details of the statistical treatment used for derivation of recommended concentrations from the collected data. That treatment, published previously (Kane and others, 1990), is summarized in table 2. This report also contains conclusions as to analytical method(s) of choice for individual elements and presents some of the more accurate methods used in the collaborative analytical programs leading to recommended concentrations for the material. Some of these methods (for example, Spies and others (chap. B, this vol.) and Roelandts (chap. C, this vol.)) are routine procedures applied without any necessary modifications due to the unique sample matrix. Other methods (for example, Wandless (chap. D, this vol.) and Huka and Rubeska (chap. E, this vol.)) require nonroutine application to obtain accurate analyses of SDO-1. The shale is the most sulfur-rich and the most organic-rich of any samples in the USGS GRS suite. The high sulfur and organic carbon contents of the material presented unique analytical problems affecting the accuracy or trueness of analysis. The analytical methods are presented here in order to assist laboratories in improving their procedures for shale analysis. 
Table 1. Summary of constituent concentrations for USGS Devonian Ohio Shale SDO-1

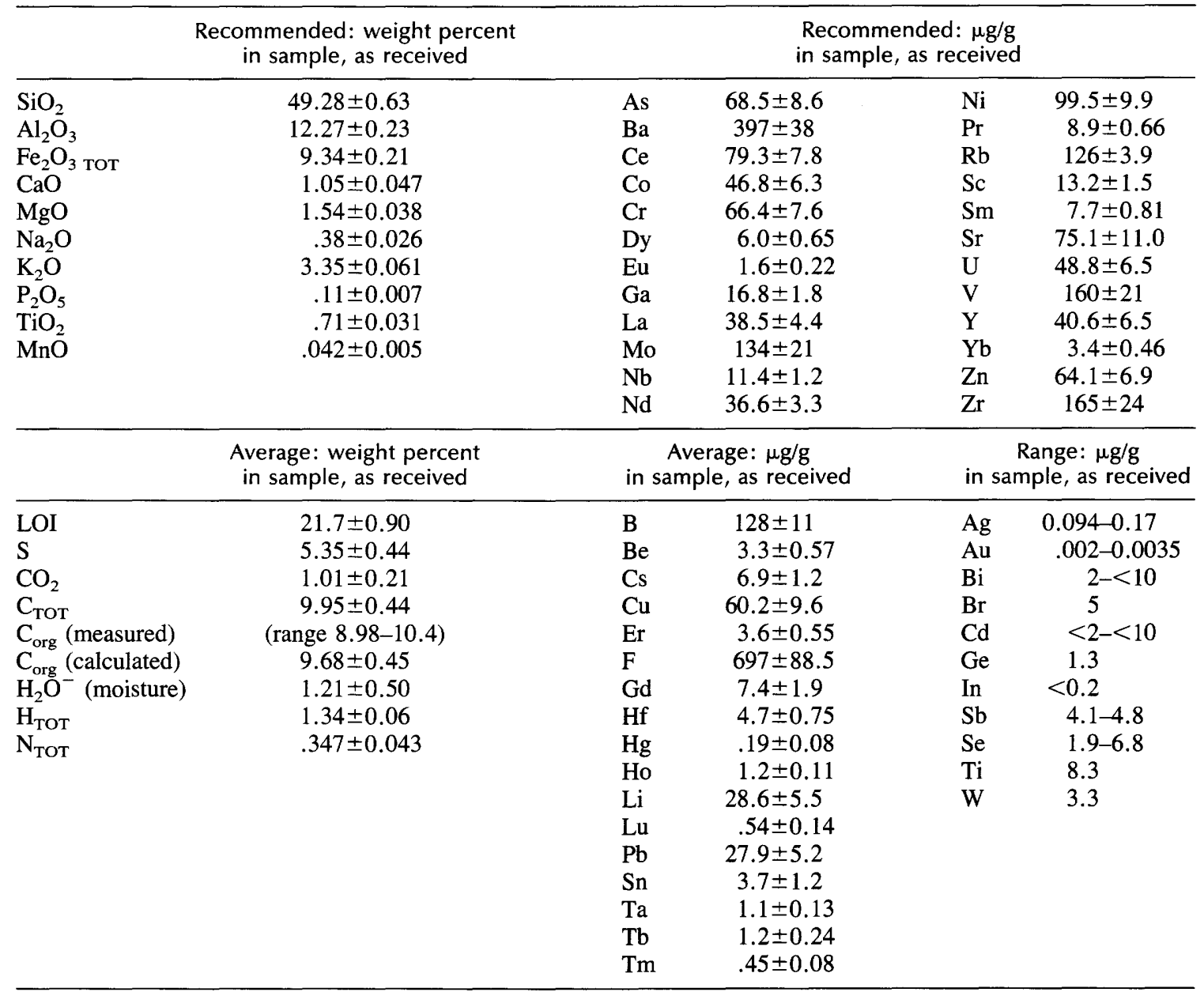

Table 2. Statistical treatment of compiled data (Kane and others, 1990, and references therein; also chap. A, this vol.)

1. Assess normalcy of data distribution of laboratory results (each of which are the average of several replicate analyses) and iteratively reject as gross outliers the individual data that lead to significant deviation from normalcy at the 95 percent confidence level.

tests: a. Arithmetic mean and median are approximately equal;

b. Distribution is neither skewed nor kurtopic; and

c. Chi-square and (or) Kolmogorov-Smirnov tests indicate normalcy.

2. Calculate mean and standard deviation for compiled data after gross outlier rejection; convert standard deviation to relative standard deviation (rsd). If rsd is 2 percent for major elements, between 5 and 10 percent for minors, or 15 percent for trace elements, proceed to step 4.

3. Test extreme individual laboratory average data against the compiled data mean $\bar{X}$ calculated in step 2 by means of a two-sided $t$-test. Reject any data shown to differ significantly at the 95-percent confidence level from $\bar{X}$. Repeat steps 1 and 2 until no further rejection is indicated.

4. Group laboratory average data surviving the rejection procedure by analytical method. Perform a one-way analysis of variance with method as the classification variable; determine significance of intermethod differences at the 95-percent confidence level.

5a. Recommend concentrations of oxide/element (1) if the final iterated $\bar{X}$ is based on at least five laboratory data determined by at least three independent methods showing no significant method differences and (2) if the rsd of $\bar{X}$ meets step 2 criteria.

5b. Cite the final iterated $\bar{X}$ as the average concentration of the oxide/element if it is based on at least five laboratory data but in some other aspect does not meet the criteria in $5 \mathrm{a}$ for a recommended concentration. Cite only a concentration range if there are fewer than five laboratory data after rejection steps $1-3$. 


\section{REFERENCES}

Arnautov, N.V., 1987, Reference samples of chemical composition of natural substances (in Russian): Novosibersk, USSR Academy of Sciences, (see K. Govindaraju, 1989).

Department of Energy, 1978, Second Eastern Gas Shales Symposium, 1978: Morgantown Energy Technology Center Report METC/SP-78/6, $461 \mathrm{p}$.

Kane, J.S., Arbogast, B.F., and Leventhal, J.S., 1990, Characterization of Devonian Ohio Shale SDO-1 as a USGS geochemical reference sample: Geostandards Newsletter, v. 14, p. 169-196.

Kepferle, R.C., de Witt, Wallace, Jr., and Flanagan, F.J., 1985, Ohio Shale (Devonian), SDO-1, from Rowan County, Kentucky: U.S. Geological Survey Open-File Report 85-145.

Provo, L.J., Kepferle, R.C., and Potter, P.E., 1978, Division of black Ohio Shale in eastern Kentucky: American Association of Petrology and Geology Bulletin, 62, p. 1703-1713. 

Chapter A

Statistical Treatment of Contributed Data in the Derivation of Recommended

Concentrations for Devonian Ohio Shale SDO-1

By JEAN S. KANE

U.S. GEOLOGICAL SURVEY BULLETIN 2046

THE USGS REFERENCE SAMPLE DEVONIAN OHIO SHALE SDO-1 



\title{
CONTENTS
}

\author{
Abstract $\mathbf{A 1}$ \\ Introduction $\mathbf{A} 1$ \\ Between-Bottle Variances A1 \\ Tests to Identify Outliers for Rejection $\mathbf{A 2}$ \\ Distribution of the Data $\mathbf{A 2}$ \\ $t$-Test for Comparison of Individual Laboratory Results With the Overall \\ Mean A3 \\ Between-Method Variance $\mathbf{A 3}$ \\ Alternative Identification of Outliers Based on Quality-Control Practices \\ Frequency of Use and Accuracy of Methods Used by Laboratories in SDO-1 \\ Analysis A6 \\ Major Oxides A6 \\ Trace Elements $\quad$ A7 \\ Updates of the Original Compilation Recommended Concentrations A11 \\ Conclusion A12 \\ References A12 \\ Appendix 1. Major/Minor Oxides: Laboratory Methods and Averages $\mathbf{A 1 4}$ \\ Appendix 2. Trace Elements: Laboratory Methods and Averages $\mathbf{A 2 0}$

\section{FIGURES} \\ 1. S-curve showing distribution of $\mathrm{SiO}_{2}$ results for SDO-1 $\mathbf{A 2}$ \\ 2. Graphs showing fit of data (cumulative frequency) to theoretical Chi-square \\ distribution and to normal distribution $\mathbf{A 3}$ \\ 3. Ce data ranges for each method of analysis A9 \\ 4. As data ranges for each method of analysis $\mathbf{A 9}$ \\ 5. Co data ranges for each method of analysis $\mathbf{A 1 0}$ \\ 6. Cr data ranges for each method of analysis $\mathbf{A 1 0}$ \\ 7. Ni data ranges for each method of analysis $\mathbf{A 1 0}$ \\ 8. $\mathrm{Cu}$ data ranges for each method of analysis $\mathbf{A 1 0}$
}

\section{TABLES}

1. Step-by-step iteration of SDO-1 Ba recommended concentration in $\mu \mathrm{g} / \mathrm{g} \quad \mathbf{A 4}$

2. Between-laboratory analysis of variance: $\mathrm{SiO}_{2} \quad \mathbf{A 5}$

3. Between-method analysis of variance: $\mathrm{SiO}_{2} \mathbf{A 5}$

4. Summary of major oxide analyses by method $\mathbf{A 6}$

5. Summary of trace element analyses by method $\mathbf{A 8}$

6. Selected rare earth elements: Comparison of results from INAA, ICP-AES after separation, ICP-MS methods A9

7. Between-method analysis of variance for trace elements whose concentrations are recommended A11

8. Examples for which newly added data do not lead to changes in recommended concentrations A12

9. Examples for which newly added data result in changes in reported concentrations except as noted $\mathbf{A 1 2}$ 



\title{
Statistical Treatment of Contributed Data in the Derivation of Recommended Concentrations for Devonian Ohio Shale SDO-1
}

\author{
By Jean S. Kane
}

\begin{abstract}
This report presents details of the statistical procedures used to derive recommended concentrations for the U.S. Geological Survey reference shale SDO-1 from data contributed by many U.S. Geological Survey and other geochemical laboratories. These include analysis of variance both to evaluate material homogeneity and to assess the significance of differences in constituent analyses between laboratories and (or) methods of analysis. Also included is a review of analytical methods used for individual elements, with evaluation of which one(s) are most accurate and precise and which are most subject to potential systematic error.
\end{abstract}

\section{INTRODUCTION}

The U.S. Geological Survey (USGS) geochemical reference sample (GRS) Devonian Ohio Shale SDO-1 (note that a Russian reference sediment sample is also called SDO-1) was first introduced as a control sample in conjunction with the Eastern Gas Shale program, 1976-1981. Publications of analytical results from that program (Leventhal and others, 1978; Department of Energy, 1978) and tentative recommended concentrations (Kepferle and others, 1985) have been followed by a number of reports (for example, Frost and others, 1985; Coveney and Glascock, 1989; Kane and others, 1990; Leventhal and Knapp, 1988) that indicate the continued geochemical interest in metalliferous black shales. One of these (Kane and others, 1990) presented recommended concentrations and their uncertainties for 40 elements, based on contributed data from more than 50 laboratories worldwide.

Analytical laboratories providing analyses for metalliferous black shale geochemical studies require an appropriately matrix-matched GRS to validate analytical methods and otherwise ensure analytical accuracy. A review of the collaborative data base for Devonian Ohio Shale SDO-1

Manuscript approved for publication September 15, 1992.
(Kane and others, 1990) indicates that although many laboratories are performing analyses with the required accuracy on SDO-1 (and presumably on metalliferous black shales, in general), some are experiencing difficulty with accurate analyses of one or several elements in samples of this type. This report examines the total data base with a view to identifying the method(s) of choice for a given element. The identification will be based on those methods showing the highest within-laboratory precision, the best between-laboratory repeatability, and lowest rejection rates in the process of deriving recommended concentrations, following the iterative procedure outlined in Kane and others (1990).

\section{BETWEEN-BOTTLE VARIANCES}

We begin the discussion with a review of the between-bottle analysis of variance (anova) presented in Kane and others (1990). Mineralogically, SDO-1 has three principal components (clay, pyrite, and organic matter) that differ considerably in density and composition. Sampling errors can be highly significant in the analyses of such mixtures. The ability to detect sampling error between bottles is a function of measurement precision for withinbottle subsamples (Kane, 1992). It is the latter which Ingamells illustrates for Cr in G-1 (Ingamells and Pitard, 1986). In this example, the within-bottle subsampling error results for the trace element $\mathrm{Cr}$ because it occurs at a very high concentration in the mineral chromite but at a much lower concentration in the bulk sample. The anorthite/orthoclase mixture described in Ingamells and Pitard (1986) demonstrates that within-bottle subsampling errors can still be significant even though much less pronounced for mixtures of major mineral phases than for dispersed minerals in bulk sample.

The basic homogeneity study for a reference sample looks primarily at sampling variance between bottles or between units-of-issue for the GRS. For SDO-1, significant bottle variances for several major oxides were found by and many collaborating laboratories (Kane and others, 1990), 


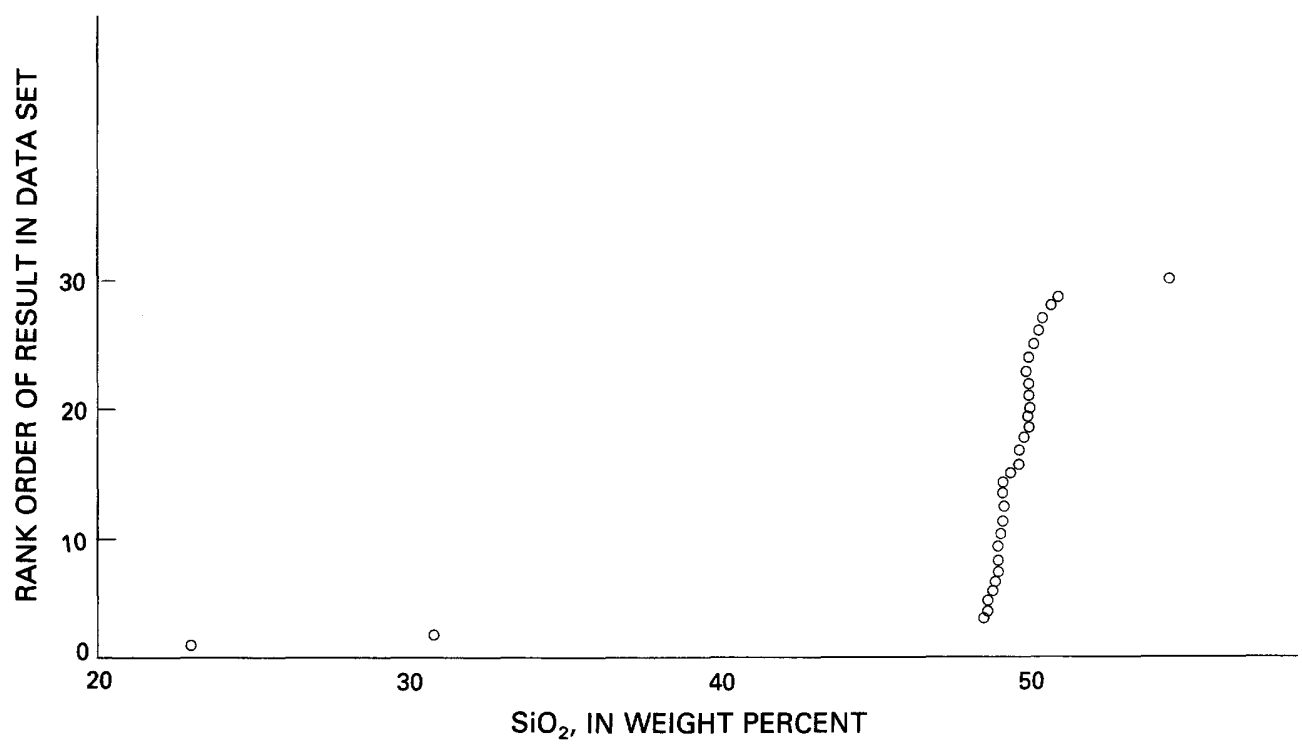

Figure 1. S-curve showing distribution of $\mathrm{SiO}_{2}$ results for SDO-1.

the between-bottle variances identified amounted to 90 percent or more of the total laboratory variance for several of those laboratories. Furthermore, these between-bottle variances appear to account for roughly 50 percent of the between-laboratory variances for the total data set, which amounted to 1-percent relative standard deviations (rsd's) for $\mathrm{SiO}_{2}, \mathrm{Al}_{2} \mathrm{O}_{3}$, and $\mathrm{Fe}_{2} \mathrm{O}_{3 \mathrm{~T}}$ and to about a 10 percent rsd for loss on ignition (LOI).

The trace elements associated with a particular principal mineral component should be expected to exhibit bottle variances parallel to those for the major oxides comprising that mineral fraction. Thus, between-bottle subsampling error would be expected to occur for $\mathrm{Cu}$ and other chalcophile trace elements associated with the pyrite phase in SDO-1 as well as for iron. However, the occurrence of within-bottle sampling variance would degrade the ability to detect between-bottle sampling variance in the same way as would the use of a relatively imprecise analytical method (Kane, 1992). None of the chalcophile trace elements were found to be heterogeneously distributed between bottles. However, many had larger standard deviations of measurement in the compiled data set than expected for the concentrations at which they occurred, presumably because of a large within-bottle subsampling component of variance. Similarly, the rare earth elements (REE's), expected to be distributed in the same manner as the clays, do not show the between-bottle variances noted for $\mathrm{Al}$ and Si. Although uranium and molybdenum, whose distributions should parallel the organic matter, are not measurably heterogeneous between bottles, LOI is heterogeneous. Therefore, sampling is thought to be a major factor in the uncertainty of compiled data averages for these elements.

\section{TESTS TO IDENTIFY OUTLIERS FOR REJECTION}

Despite the reality of a significant sampling component in the between-laboratory/method analysis of variance for some elements, what follows will neglect that subsampling error. We will instead attempt to discern the other causes of interlaboratory and intermethod disagreements, and focus on those.

\section{Distribution of the Data}

The iterative process by which contributed data are accepted or rejected begins with an evaluation of the normalcy of the distribution of submitted data. Assuming that no bias affects any contributed data, but that all error is truly random, the contributed data should have a normal distribution (Deming, 1943; Youden, 1960). Several measures of normalcy of the distribution can be made, each having limitations related to the size of the data set. These measures are the agreement between the arithmetic mean and the median, the coefficients of skewness $\left(V b_{1}\right)$ and of kurtosis $\left(\mathrm{b}_{2}\right)$, chi-square $\left(\mathrm{X}^{2}\right)$ and the Kolmogorov-Smirnov maximum deviation (D) (Dixon and Massey, 1983; Lister, 1982; Deming, 1943; Siegel, 1956). The data for $\mathrm{SiO}_{2}$ before any rejection is both negatively skewed and leptokurtic. The mean and median differ in the second significant figure, and the standard deviation of the mean indicates that error begins in the third significant figure. Both $\mathrm{X}^{2}$ and $\mathrm{D}$ indicate less than 1-percent probability of the distribution being normal. Visual inspection of a simple S-curve distribution of the data (fig. 1) readily reveals the outliers to be the two lowest and one highest compiled values. With their removal, all measures of goodness of fit indicate that the distribution is normal. The mean and median now differ 

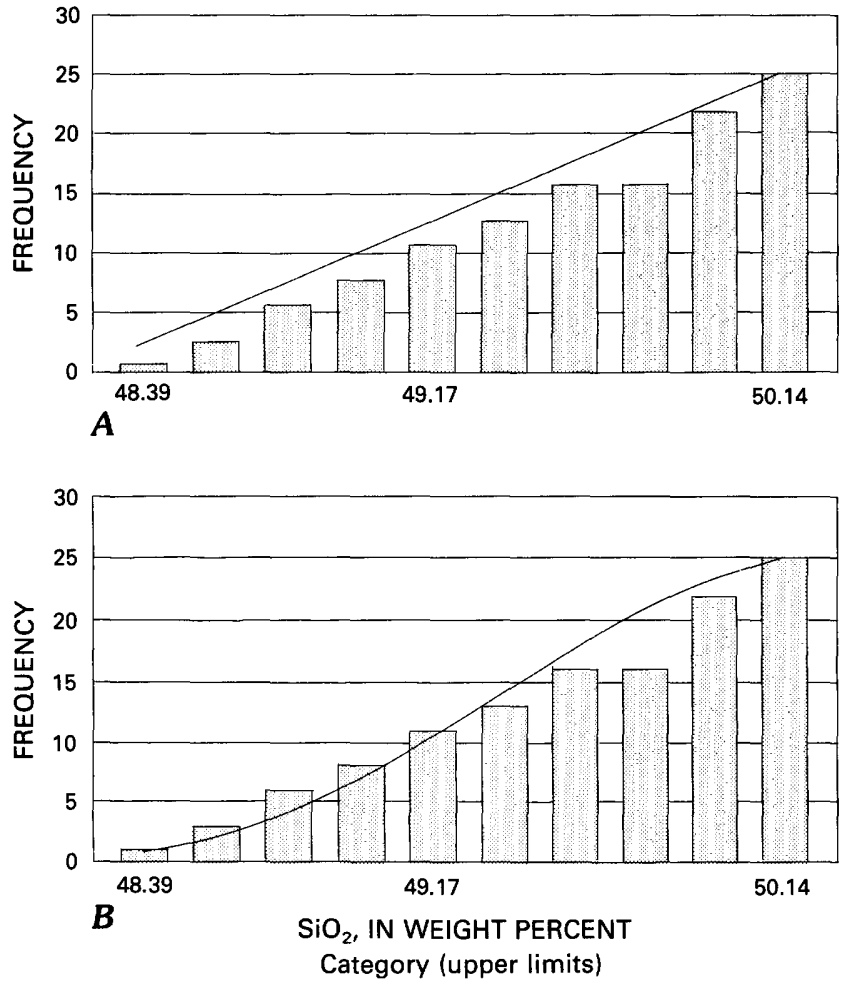

Figure 2. Fit of data (cumulative frequency) to $(A)$ theoretical Chi-square distribution and $(B)$ to normal distribution.

only in the fourth significant figure. The fit of the data to theoretical $\mathrm{X}^{2}$ and normal distributions are shown in figures $2 A, 2 B$.

\section{$t$-Test for Comparison of Individual Laboratory Results With the Overall Mean}

Once the data distribution is normal, the high and jlow individual laboratory averages $\left(\bar{X}_{i}\right)$ are compared to the iterated mean $\left(\bar{X}_{T}\right)$ using a $t$-test (Dixon and Massey, 1983; Lister, 1982). This rejection procedure can be overly severe when $n$ is large, and inadequately rigorous when $n$ is small. For example, at $n=30$ rejection occurs at $\left|\bar{X}_{i}-\bar{X}_{T}\right|=0.5 s$, but at $n=3$ it occurs at $\left|\bar{X}_{i}-\bar{X}_{T}\right|$ $=3 \mathrm{~s}$, where $s$ is the standard deviation of $\bar{X}_{T}$. Particularly in view of the bottle inhomogeneities already noted, excessive rejection must be avoided to arrive at realistic uncertainty estimations for the recommended concentrations.

Returning to the $\mathrm{SiO}_{2}$ data set, once the data distribution was normal after eliminating the two lowest and one highest results, the interlaboratory mean had an rsd of 1.3 percent, within the criteria range for a recommended major-oxide concentration. $t$-test criteria for $n=27$ would reject individual laboratory results deviating from the overall average by only $0.56 \mathrm{~s}$, but this value approaches the bottle-to-bottle variation identified in the homogeneity study. Several successive iterations based on the $t$-test criteria have little to no effect on either the final mean or the final uncertainty of that value $(49.28 \pm 0.63$ percent at $n=27$, rsd $1.3 ; 49.27 \pm 0.49$ percent at $n=19$, rsd 1.0 ). $t$-test rejection is therefore not carried out. Instead, method analysis of variance is performed, as discussed in the next section, and the value of $49.28 \pm 0.63$ percent is recommended for $\mathrm{SiO}_{2}$ in SDO-1.

The $\mathrm{Ba}$ data set will illustrate the case where all parameters, with the possible exception of $\mathrm{X}^{2}$, showed the initial distribution to be normal. No data were rejected through data distribution tests, but six laboratory results were rejected based on $t$-tests. The procedure and results are shown in table 1 . The initial $\bar{X}_{T}$ for Ba is $440 \pm 117 \mu \mathrm{g} / \mathrm{g}$ for $n=26$. Despite normalcy of the distribution, the uncertainty of the interlaboratory average (rsd 27 percent) is unacceptably large. $t$-test rejection occurred pairwise for the high and low extreme values, which differed by more than $0.56 \mathrm{~s}(66 \mu \mathrm{g} / \mathrm{g})$ from $\bar{X}_{T}$. Iteration gave a new $\bar{X}_{T}$ of $437 \pm 93 \mu \mathrm{g} / \mathrm{g}$ for $n=24$. At this point, reevaluation of the distribution indicated deterioration; all parameters were closer than initially to critical values at which the hypotheses of normal distribution would be rejected. Additionally, the magnitude of the rsd ( 22 percent) required further rejection. After the next two cycles of pairwise $t$-testing that led to one-sided rejection of high values, skewness was serious, $\mathrm{X}^{2}$ also indicated that the distribution was not normal, and the rsd (18 percent) was still too large to permit recommending the interlaboratory average $\mathrm{Ba}$ concentration of $419 \mu \mathrm{g} / \mathrm{g}$. Three further $t$-test iteration cycles, again rejecting only high values, led to $\bar{X}_{T}=394 \pm 37 \mu \mathrm{g} / \mathrm{g}$ for $n=19$. Iteration was then suspended since the distribution was then normal under all tests, and the rsd of 9.4 percent met procedural criteria.

\section{Between-Method Variance}

Intermethod agreement, the final criterion for recommending an elemental concentration, parallels the National Institute of Standards and Technology (NIST) certification procedures (Uriano and Gravatt, 1977; Natrella, 1963; Cali and Reed, 1974; Michaelis, 1975), which require betweenmethod agreement for the certification of concentrations in standard reference materials (SRM's). These certified concentrations represent the closest possible estimation of "true value," an ideal that all analyses attempt to approach but nonetheless remains unknown (Youden, 1960; International Organization for Standardization (ISO), 1978, 1989). We accept the hypothesis that method averages are the same when that is true at the 95 percent confidence level, that is, when there is $\leq 5$ percent probability that the method averages are statistically different.

In the SDO-1 and most other compiled data bases, significant between-laboratory variances are the rule, not the exception, where data permits their evaluation. This variance is not entirely unexpected (Youden, 1960; 
Table 1. Step-by-step iteration of SDO-1 Ba recommended concentration in $\mu \mathrm{g} / \mathrm{g}$

[rds, relative standard deviation; NS, not significant; (S), significant at $95 \%$, but not at $99 \%$, confidence level; S, significant at both $95 \%$ and $99 \%$ confidence level]

\begin{tabular}{|c|c|c|c|c|c|c|c|}
\hline \multirow[b]{2}{*}{$\begin{array}{l}\text { Mean and } \\
\text { standard } \\
\text { deviation (rsd) }\end{array}$} & \multirow[b]{2}{*}{$\begin{array}{l}\text { Number } \\
\text { using: } \\
\text { Data }\end{array}$} & \multicolumn{5}{|c|}{ Test for normal distribution } & \multirow[b]{2}{*}{ Decision/comment } \\
\hline & & Median & Skewness & Kurtosis & $\begin{array}{l}\text { Kolmogorov- } \\
\text { Smirnov (D) } \\
\text { (Probability d }\end{array}$ & $\begin{array}{l}\text { Chi-square } \\
\qquad\left(\mathrm{X}^{2}\right) \\
\text { ist. is normal) }\end{array}$ & \\
\hline $440 \pm 117(27 \%)$ & 26 & 406 & NS & NS & $0.227(0.14)$ & $15.48(0.000)$ & $\begin{array}{l}\text { Four of five tests show distribution } \\
\text { normal; however, rsd exceeds limit, } \\
\text { and } t \text {-test of extreme high and low } \\
\text { values leads to their rejection. }\end{array}$ \\
\hline $428 \pm 102(24 \%)$ & 25 & 404 & NS & NS & $.205(.247)$ & $14.04(.000)$ & \\
\hline $437 \pm 94 \quad(22 \%)$ & 24 & 406 & (S) & NS & $.263(.063)$ & $12.08(.007)$ & $\begin{array}{l}\text { rsd still unacceptable and distribution } \\
\text { deteriorating; continue } t \text {-test rejec- } \\
\text { tion. }\end{array}$ \\
\hline $428 \pm 85(20 \%)$ & 23 & 404 & $S$ & NS & $.255(.098)$ & $8.77(.032)$ & \\
\hline $419 \pm 74(18 \%)$ & 22 & 401 & $S$ & NS & $.228(.202)$ & $6.91(.032)$ & $\begin{array}{l}\text { Rejection is one-sided (high values } \\
\text { only) at this point; skewness is sig- } \\
\text { nificant; with each new rejection, D } \\
\text { and } \mathrm{X}^{2} \text { show increasing probability } \\
\text { that distribution is normal. }\end{array}$ \\
\hline $419 \pm 63(15 \%)$ & 21 & 399 & $S$ & NS & $.193(.413)$ & $5.05(.080)$ & \\
\hline $401 \pm 47(12 \%)$ & 20 & 394 & NS & NS & $.117(.946)$ & $2.86(.240)$ & $\begin{array}{l}\text { Rsd remains unacceptable until skew- } \\
\text { ness is removed. } t \text {-test indicates } \\
\text { unacceptability of high value. }\end{array}$ \\
\hline $394 \pm 37(9.4 \%)$ & 19 & 388 & NS & NS & $.088(.998)$ & $.249(.618)$ & $\begin{array}{l}\text { At } n=19, \mathrm{rsd}=9.4 \text { percent and distri- } \\
\text { bution is normal. Criteria to recom- } \\
\text { mend concentration are met. }\end{array}$ \\
\hline $398 \pm 33(8.3 \%)$ & 18 & 394 & NS & NS & & & $\begin{array}{l}t \text {-tests indicate further rejection, but } \\
\text { iterated concentrations change only } \\
\text { slightly; further iteration is unwar- } \\
\text { ranted. }\end{array}$ \\
\hline $394 \pm 29(7.4 \%)$ & 17 & 388 & NS & NS & & & \\
\hline
\end{tabular}

Bastenaire, 1979), as subtle bias is likely to affect even the most carefully developed and applied analytical method. Interlaboratory differences are evaluated with a standard anova procedure using laboratory rather than bottle as the classification variable. Complete evaluation requires that all contributing laboratories submit individual analytical results, and not just laboratory averages, so that withinlaboratory or within-method variance can be calculated.

Although 11 laboratory averages for $\mathrm{SiO}_{2}$ based on X-ray fluorescence (XRF) analysis survived the iterative rejection process, only four of those laboratories reported individual results in addition to the final laboratory average. For those four laboratories, the between-laboratory anova shows highly significant differences, as seen in table 2. Similarly, three laboratories using some variation of the Shapiro and Brannock (1962) "rapid rock" procedure obtained significantly different results. The same is true for three other laboratories using inductively coupled plasmaatomic emission spectrometry (ICP-AES) after an unspecified decomposition and for two laboratories using colorimetry (table 2 ).

However, when method rather than laboratory becomes the classification variable and interlaboratory method averages are compared, no significant differences are found. The method averages for $\mathrm{SiO}_{2}$, based on XRF $(n=11)$, colorimetric $(n=2), \mathrm{dc}$ arc AES $(n=1)$, and "rapid rock" ( $n=3)$ analyses, are the same, with $>95$-percent probability, as shown in table 3 . The intermethod average of $49.28 \pm 0.63$ percent $\mathrm{SiO}_{2}$ is designated a recommended concentration because both the intermethod agreement and the magnitude of the interlaboratory relative standard deviation (rsd) meet USGS criteria for recommended concentrations (Kane and others, 1990). Again, we point out that roughly half of the final interlaboratory rsd is due to bottle differences.

A similar evaluation process led to recommended concentrations for all rock-forming major oxides measured in SDO-1. For most major oxides other than $\mathrm{SiO}_{2}$, the iteration proceeded through several cycles, removing either a single point or paired high and low outliers as appropriate, before resulting in an acceptable, normal data distribution.

For the $\mathrm{Ba}$ data set, five methods of analysis were also used by contributing laboratories; the anova procedure resulted in an 82 percent probability that results from all five methods were the same. Therefore, the Ba concentration was also recommended.

Similarly, concentrations were recommended for 23 other trace elements based on the outlined procedure. 
Table 2. Between-laboratory analysis of variance: $\mathrm{SiO}_{2}$ in weight percent

\begin{tabular}{lrcccc}
\hline $\begin{array}{c}\text { Method/ } \\
\text { laboratory }\end{array}$ & $\begin{array}{c}\text { Laboratory mean } \\
\text { and standard deviation }\end{array}$ & $\begin{array}{c}\text { Degrees of } \\
\text { freedom }\end{array}$ & F-ratio ${ }^{1}$ & $\begin{array}{c}\text { Probability laboratory } \\
\text { means do not differ }\end{array}$ \\
\hline XRF & 2 & $48.43 \pm 0.59$ & 4,21 & 29.72 & 0.0000 \\
& 5 & $50.05 \pm 0.09$ & & & \\
& 6 & $48.57 \pm 0.52$ & & & \\
& 15 & $50.16 \pm 0.077$ & & & \\
Fusion & 17 & $49.54 \pm 0.067$ & & & \\
ICP-AES & 1 & $50.05 \pm 0.12$ & & & \\
& 4 & $48.68 \pm 0.22$ & & & \\
Color & 16 & $48.77 \pm 0.23$ & & & \\
& 14 & $49.45 \pm 0.16$ & 1,10 & 51.04 & \\
& 15 & $48.73 \pm 0.17$ & & & \\
\hline
\end{tabular}

${ }^{1} \mathrm{~F}$-ratio $=\frac{\text { variance between laboratories }}{\text { variance within laboratories }}$

Table 3. Between-method analysis of variance anova: $\mathrm{SiO}_{2}$

\begin{tabular}{lccc}
\hline & $\begin{array}{c}\text { Method } \\
\text { mean and } \\
\text { standard } \\
\text { deviation }\end{array}$ & $\begin{array}{c}\text { Number of } \\
\text { laboratories } \\
\text { using } \\
\text { method }\end{array}$ & Anova data \\
\hline XRF & $49.12 \pm 0.47$ & 11 & $\begin{array}{c}\text { Degrees of } \\
\text { freedom }=3,13\end{array}$ \\
$\begin{array}{l}\text { Fusion ICP-AES } \\
\text { Color }\end{array}$ & $49.29 \pm 0.71$ & 3 & F-ratio $=0.115$ \\
dc arc AES & $48.97 \pm 0.39$ & 2 & $\begin{array}{l}\text { Probability } \\
\text { method means do } \\
\text { not differ }=0.97\end{array}$ \\
\hline
\end{tabular}

Average values, which did not meet criteria for recommended values, were derived for $\mathrm{LOI}$, moisture, $\mathrm{CO}_{2}, \mathrm{C}_{\text {org }}$, $\mathrm{C}_{\mathrm{TOT}}$, and 16 trace elements.

\section{Alternative Identification of Outliers Based on Quality-Control Practices}

Rejection of data could be more appropriately based on quality-control $(\mathrm{QC})$ evidence rather than statistical tests by referencing a method standard operating procedure (SOP). For example, if the SOP reported that the routinely achievable accuracy of the method did not meet the criteria for recommended concentrations, data obtained using the procedure would be rejected. QC evidence could also be provided by reporting accurate data for an appropriate, previously characterized reference sample. Since neither option was available in characterizing SDO-1, statistical procedures alone were relied on in determining which data to reject or accept in the final data set.

$\mathrm{Ba}$ data provides an excellent illustration of the desirability of QC criteria for rejection. In another paper in this volume (chap. D), Wandless discusses the large interference that $U$ can cause in the $B a$ result in instrumental neutron activation analysis (INAA). In fact, several of the rejected high values in the data set were INAA values; the correction indicated in the Wandless paper would bring all rejected values within the uncertainty of the recommended concentration. SOP's from each of the contributing laboratories would be required, however, to identify whether or not this factor actually explained the high INAA results that were rejected. Acid decomposition prior to ICP-AES might be expected to result in low Ba results, or at least to more within-method variation than in a fusion data set, because of sulfur oxidation to sulfate and potential precipitation of $\mathrm{Ba}$ during the decomposition. The QC approach allows the underlying causes of poor data to be identified and, thus, permits correction of procedures to eliminate the error. Statistical procedures alone do not accomplish this aim.

It is not always obvious how best to identify methods. The original rapid-rock procedure (Shapiro and Brannock, 1962) used several single-element colorimetric or flame atomic absorption determinations after a fusion decomposition of the sample; today, ICP-AES substitutes for most of those other final measurements. Is a single, broad method specification of rapid rock sufficient for all of these variations? ICP-AES preceded by acid decomposition is in some ways distinctly different from ICP-AES preceded by fusion decomposition, but in other ways the two are identical. ICP-AES and dc arc AES are distinctly different in that one analyzes sample after decomposition and the other analyzes sample without prior decomposition. The two methods have different excitation mechanisms, and excitation occurs at different temperatures, but in the final analysis both are based on the single atomic emission principle. We have used rather broad classifications rather than extremely narrow ones, while maintaining a distinction between solid sample and solution analysis.

Ideally, at least three methods of analysis have been used to analyze both solid and decomposed sample, and the methods differ in broad classification: X-ray spectrometry, atomic (absorption/ emission) spectrometry, instrumental neutron activation analysis, mass spectrometry, classical wet chemistry, and others. With few exceptions, most 
Table 4. Summary of major oxide analyses by method

[( ), reported, but rejected; leaders $(-)$, none reported]

\begin{tabular}{|c|c|c|c|c|c|c|c|c|}
\hline \multirow{2}{*}{$\begin{array}{c}\text { Total } \\
\text { number } \\
\text { of data } \\
\text { accepted }\end{array}$} & \multirow[b]{2}{*}{ Oxide } & \multicolumn{7}{|c|}{ Number of accepted analyses that were determined by: } \\
\hline & & WDXR & $\begin{array}{l}\text { Fusion } \\
\text { ICP-AES }\end{array}$ & $\begin{array}{l}\text { Unknown decomp } \\
\text { ICP-AES }\end{array}$ & INAA & AAS & $\begin{array}{l}\text { dc arc } \\
\text { AES }\end{array}$ & Color \\
\hline 26 & $\mathrm{SiO}_{2}$ & 11 & 3 & 2 & - & - & 1 & 1 \\
\hline 23 & $\mathrm{Al}_{2} \mathrm{O}_{3}$ & 11 & 2 & 6 & () & 4 & ( ) & 1 \\
\hline 26 & $\mathrm{Fe}_{2} \mathrm{O}_{3 \text { Tот }} *$ & 10 & 3 & 5 & 2 & 4 & 2 & ( ) \\
\hline 24 & $\mathrm{MgO}$ & 8 & 3 & 4 & () & 4 & 1 & - \\
\hline 27 & $\mathrm{CaO}$ & 10 & 3 & 4 & ( ) & 3 & 1 & - \\
\hline 24 & $\mathrm{Na}_{2} \mathrm{O}$ & 7 & 3 & 4 & 3 & 3 & 2 & - \\
\hline 22 & $\mathrm{~K}_{2} \mathrm{O}$ & 10 & 3 & 3 & 2 & 3 & ( ) & - \\
\hline 25 & $\mathrm{TiO}_{2}$ & 10 & 3 & 3 & () & () & - & 1 \\
\hline 20 & $\mathrm{P}_{2} \mathrm{O}_{5}$ & 8 & 2 & 5 & ( ) & ( ) & ( ) & 1 \\
\hline \multirow[t]{3}{*}{22} & $\mathrm{MnO}$ & 7 & 3 & 6 & ( ) & 4 & 2 & ( ) \\
\hline & & \multicolumn{7}{|c|}{ Number of rejected analyses that were determined by: } \\
\hline & & WDXR & $\begin{array}{l}\text { Fusion } \\
\text { ICP-AES }\end{array}$ & $\begin{array}{l}\text { Unknown decomp } \\
\text { ICP-AES }\end{array}$ & INAA & AAS & $\begin{array}{c}\text { dc arc } \\
\text { AES }\end{array}$ & Color \\
\hline & $\mathrm{SiO}_{2}$ & 0 & 0 & 0 & - & - & 1 & 1 \\
\hline & $\mathrm{Al}_{2} \mathrm{O}_{3}$ & 2 & 1 & 1 & 2 & 1 & 1 & 1 \\
\hline & $\mathrm{Fe}_{2} \mathrm{O}_{3 \mathrm{TOT}} *$ & 0 & 0 & 0 & 3 & 1 & 0 & 1 \\
\hline & $\mathrm{MgO}$ & 1 & 0 & 2 & 1 & 0 & 0 & - \\
\hline & $\mathrm{CaO}$ & 0 & 0 & 2 & 1 & 0 & 1 & - \\
\hline & $\mathrm{Na}_{2} \mathrm{O}$ & 3 & 0 & 1 & 0 & 1 & 0 & - \\
\hline & $\mathrm{K}_{2} \mathrm{O}$ & 0 & 0 & 2 & 1 & 0 & 2 & - \\
\hline & $\mathrm{TiO}_{2}$ & 0 & 0 & 0 & 2 & - & - & 0 \\
\hline & $\mathrm{P}_{2} \mathrm{O}_{5}$ & 1 & 1 & 1 & - & - & - & 0 \\
\hline & $\mathrm{MnO}$ & 1 & 0 & 0 & - & 0 & 0 & - \\
\hline \multicolumn{2}{|c|}{$100 \times \frac{\text { total number rejected for method }}{\text { total number accepted for method }}$} & $9 \%$ & $7 \%$ & $24 \%$ & $140 \%$ & $12 \%$ & $78 \%$ & $75 \%$ \\
\hline
\end{tabular}

*Total iron reported as $\mathrm{Fe}_{2} \mathrm{O}_{3}$; occurs as $\mathrm{FeS}_{2}, \mathrm{FeCO}_{3}$ or $\mathrm{FeO}$ (Leventhal and Hosterman, 1982)

notably LOI and $\mathrm{H}_{2} \mathrm{O}$ (tot), this goal is readily achievable for major oxides but much more difficult to achieve for trace constituents, as will be seen shortly.

\section{FREQUENCY OF USE AND ACCURACY OF METHODS USED BY LABORATORIES IN SDO-1 ANALYSIS}

Based on the method averages from which recommended concentrations were derived, we can draw conclusions as to (1) frequency of use, or popularity, of specific methods and (2) general acceptability of each of those methods in terms of accuracy and precision (or in ISO (1978) terminology, trueness and repeatability) for each constituent measured. Based on the percentage of laboratory averages obtained by any acceptable method that had to be rejected during the iteration leading to the recommended concentration, some conclusion regarding ease of application or trouble-free nature of the method can also be drawn. We now examine the compiled data with respect to these conclusions. In doing so we must note that a sizeable number of laboratories did not specify the method of analysis used.

\section{Major Oxides}

Approximately 70 percent of the laboratories reporting data specified the analytical method used; from that specification, it was determined that X-ray fluorescence and atomic spectrometry provided the bulk of the major constituent data, as shown in table 4. Most laboratories using XRF for these analyses used the wavelength-dispersive method (WDXRF) and fused the samples into glass beads or discs prior to X-ray excitation, as described, for example, by Spies and others (chap. B, this vol.). Energy-dispersive $\mathrm{X}$-ray analysis of pressed powder (EDXRF) was used by only one laboratory (Kopp and Furman, chap. G, this vol.). The standard protocol developed for clays and soils in Kopp's laboratory was inadequate in correcting for the shale matrix effects encountered in analyzing SDO-1 (Kopp and Furman, chap. G, this vol.). Since less than 10 percent of 
all WDXRF data in the compilation was rejected during the iterative process leading to recommended concentrations, it can be concluded that the WDXRF method is highly repeatable between laboratories and readily applied with accuracy to samples of the shale matrix. The method is particularly advantageous because it permits determination of sulfur along with other major constituents. During ignition prior to fusion, most sulfur is lost, but some is retained in ignited sample (Kane and Skeen, chap. F, this vol.). Measurement of both retained sulfur in ash and LOI is important if oxide summations are used to assess acceptability of the analysis. However, the nature of the shale sample introduces considerably more error in the oxide summation evaluation than that which occurs for typical igneous rocks (Lechler and Desilets, 1987), as discussed elsewhere in this volume (Kane and Skeen, chap. F, this vol.; Huka and Rubeska, chap. E, this vol.). Also, it must be recognized that the reporting of total iron as ferric oxide, following normal rock analysis convention, accurately reflects the iron occurrence in the ignited and fused XRF analytical sample, but not in the shale itself (Leventhal and Hosterman, 1982). This reporting convention also affects the oxide summation of the recommended concentrations for the reference sample itself, although not for the ignited residue that is analyzed by XRF.

Atomic spectrometry can be subdivided into emission (AES) and absorption (AAS) spectrometries, and each of these can be subdivided as well. For example, subdivision of AES by excitation process would result in inductively coupled plasma (ICP-AES), direct current plasma (DCPAES), and dc arc AES methods. In this work, each was treated as a different method for the method anova statistics. Also, both AES and AAS could be subdivided depending on whether or not prior sample decomposition was required for analysis, leading to solid/flux, acid decomposition, fusion decomposition categories for the method anova. Although this subdivision led to several distinct atomic spectrometric methods for method anova, intermethod agreement was not considered sufficient for purposes of recommending concentrations if all methods were fundamentally atomic spectrometric methods. A totally independent method, for example, XRF, INAA, or gravimetry, in agreement with the atomic spectrometric methods, was required. Based on low rejection rates indicating overall accuracy of submitted data, fusion ICP-AES is a method of choice for major constituents; within-laboratory precision for some oxides is slightly poorer than for WDXRF, and S cannot be included in the analysis; but otherwise the two methods are equally suited to accurate and precise characterization of reference samples for their major constituents.

Acid (or unspecified) decomposition ICP-AES and acid decomposition AAS were also applied successfully to SDO- 1 analysis, as was flame photometry for $\mathrm{Na}$ and $\mathrm{K}$. The obvious disadvantage of any analysis following acid decomposition is the inability to provide $\mathrm{SiO}_{2}$ results. For some major constituents, chemical methods including colorimetry, volumetric analysis, and gravimetry were used by a small number of contributors, as were INAA analyses. Combustion/coulometry was the primary method for carbon and sulfur analyses. Chemical analyses and INAA, neither of which show the robustness between laboratories of WDXRF and atomic spectrometry, were used less frequently and had higher rejection rates and larger betweenlaboratory rsds for data surviving the rejection process than the optimum methods.

\section{Trace Elements}

Intermethod comparisons of trace element determinations are more difficult to generalize than those for major oxides. A wider range of methods are used, but the extent of overlapping coverage of specific elemental suites between methods is much more limited, as indicated in table 5. For example, INAA is used to provide a sizeable portion of the REE data; XRF analyses are also used extensively to provide $\mathrm{Ce}$ and $\mathrm{La}$ analyses, but no other REE data. However, direct ICP-AES without prior group separations and AAS are seldom used to provide any REE data. On the other hand, those atomic spectroscopic methods provide a major portion of data for such elements as $\mathrm{Cu}$, $\mathrm{Li}$, and $\mathrm{Pb}$ where INAA data are totally absent. Confirmation of values by several independent techniques was readily achieved for the major oxides but much harder to accomplish for the traces. Also, the data sets for each trace element were generally smaller (average $n=9$ ) than those for the majors (average $n=20$ ) except in a relatively few instances. We have already examined the intermethod $\mathrm{Ba}$ data in detail, while discussing the rejection process and recommended value derivation. We now will look at a number of other trace elements in similar detail.

Although INAA methods historically dominated in the determination of REE, IDMS has long been considered the method of choice for the odd atomic weight REE. However, even in data bases for reference samples like BCR-1 and BHVO-1 used throughout the U.S. space program, the number of IDMS data points available have represented only a small fraction (18 percent for BCR-1 and 5 percent for BHVO-1) of the total data set (Flanagan, 1976; Gladney and others, 1983). More recently, both ICP-AES following group separation and ICP-MS methods have been developed for the REE, making intermethod validation of REE recommended concentrations possible. Table 6 shows that no clear superiority of one method over the others results from F-tests comparing method variances or $t$-tests comparing the several method averages to one another for most of the REE. Larger data sets, however, would improve the rigor of the tests and might show subtle differences between methods results that are not statistically significant in the current data set. A case in point is $\mathrm{Ce}$, for 
Table 5. Summary of trace element analyses by method [leaders (-), none reported by that method; sepn., separation]

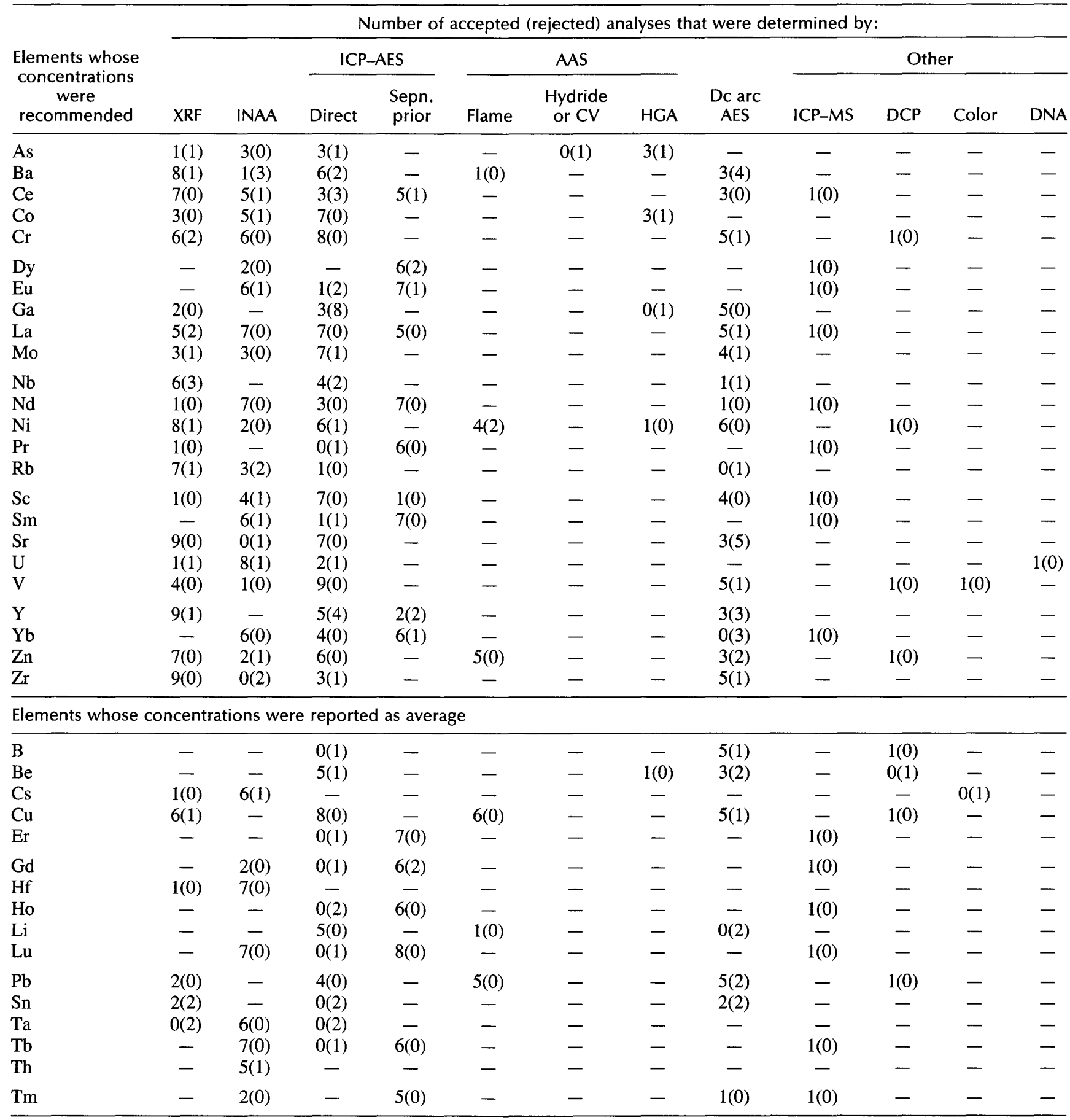

which the individual method ranges are plotted in figure 3. Apparently, solution methods (ICP-AES, with and without separation, and ICP-MS) produce results that are slightly lower than solid-sample methods (INAA, XRF, dc arc AES). Ce is known to occur in minerals resistant to acid attack, which was used almost exclusively in obtaining the solution-method results. Results from all six methods are the same, having a 33-percent probability; however, the probabilities increase to 55 percent and 88 percent, looking for agreement between either the solution (ICP-AES, ICP-MS) or the solid-sample methods (dc arc AES, XRF, INAA).

In deriving the recommended concentration for As, the last iteration acceptance range was $52-84 \mu \mathrm{g} / \mathrm{g}$. Intermethod agreement exists with a probability of only 3 percent between XRF, ICP-AES, INAA, and hydride 
Table 6. Selected rare earth elements: Comparison of results in $\mu \mathrm{g} / \mathrm{g}$ from INAA, ICP-AES after separation, ICP-MS methods

[S, significant at $95 \%$ confidence; NS, not significance at $95 \%$ confidence]

\begin{tabular}{lccccc}
\hline Element & INAA & $\begin{array}{c}\text { Ion exch. Sepn. } \\
\text { ICP-AES }\end{array}$ & ICP-MS & $\begin{array}{c}\text { Anova* for } \\
\text { differences } \\
\text { in precision }\end{array}$ & $\begin{array}{c}t \text {-Test for } \\
\text { differences } \\
\text { in means }\end{array}$ \\
\hline $\mathrm{Ce}$ & $78.1 \pm 6.1$ & $74.0 \pm 2.8$ & $73.2 \pm 1$ & $\mathrm{NS}$ & $\mathrm{NS}$ \\
$\mathrm{Nd}$ & $36.6 \pm 3.2$ & $37.4 \pm 1.6$ & 7.0 & $\mathrm{NS}$ & $\mathrm{NS}$ \\
$\mathrm{Sm}$ & $7.8 \pm 0.40$ & $7.8 \pm 0.81$ & 6.8 & $\mathrm{NS}$ & $\mathrm{NS}$ \\
$\mathrm{Eu}$ & $1.74 \pm 0.18$ & $1.65 \pm 0.23$ & 1.71 & $\mathrm{NS}$ & $\mathrm{NS}$ \\
$\mathrm{Gd}$ & $7.82 \pm 0.78$ & $6.55 \pm 0.49$ & 6.9 & $\mathrm{NS}$ & $\mathrm{NS}$ \\
$\mathrm{Tb}$ & $1.07 \pm 0.19$ & $1.28 \pm 0.19$ & .80 & $\mathrm{NS}$ & $\mathrm{NS}$ \\
$\mathrm{Lu}$ & $.63 \pm 0.16$ & $.45 \pm 0.02$ & .42 & $\mathrm{NS}$ & $\mathrm{NS}$ \\
\hline
\end{tabular}

*Omits ICP-MS

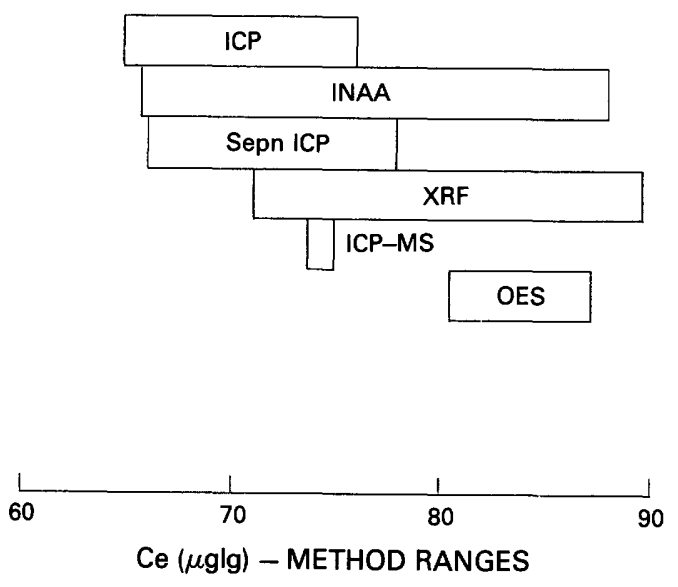

Figure 3. Ce data ranges for each method of analysis.

generation-AAS method means, but within each method group between-laboratory relative standard deviations of approximately 10 percent are found. If, we plot the full range of As data before rejection by method (fig. 4), we would intuitively reject the XRF method as providing data which differs from that for the other three methods. Repeating the between-method anova after removing the one XRF result previously retained reveals that the probability that the three method averages from ICP-AES, INAA, and hydride-AAS are the same improves considerably, to 43 percent. Although two methods require sample dissolution, one does not, which suggests no decomposition error. Two totally different classes of method, atomic spectrometry and nuclear, are in agreement. The intermethod average can therefore be recommended, having fair confidence that no method-specific error source is affecting its reliability.

On the other hand, few of the individual laboratory results for As agree with one another if the anova is performed between laboratories rather than between methods, as discussed earlier. A considerable part of the discrepancy is probably attributable to calibration protocol

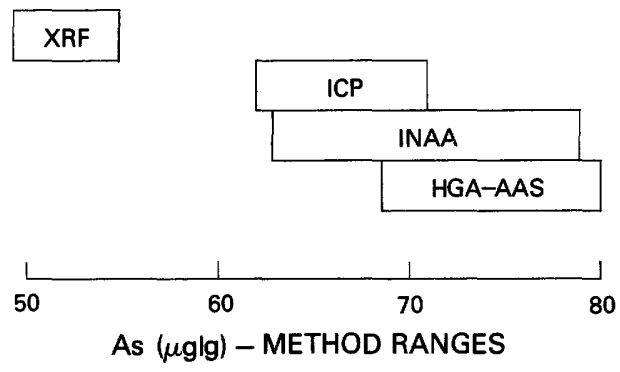

Figure 4. As data ranges for each method of analysis.

differences. INAA and XRF in particular are often calibrated with secondary standards, and uncertainties in the calibration standard values can be a significant factor in the overall analytical error for each laboratory. A notable absence of hydride generation data for the sample suggests difficulties with that method in analyzing organic-rich (and (or) sulfur-rich) samples. One late addition to the data set, in fact, was a hydride generation As result of $38 \mu \mathrm{g} / \mathrm{g}$, the lowest result submitted. There is some indication that the observed bias may be due to the presence of As in an organo-metallic complex that does not decompose under the conditions used to prepare samples for hydride generation (A. Horowitz, personal commun., 1990).

Because the concentration of Se in uraniferous shales is sometimes enriched considerably over that in most geochemical samples (Schultz and others, 1980) and additional Se reference samples are environmentally desirable, it is unfortunate that so few (3) laboratories contributed $\mathrm{Se}$ data for SDO-1. The range of those results, however, implies that SDO-1 contains less Se than many shales, approximately $5 \mu \mathrm{g} / \mathrm{g}$ in comparison to as much as $300 \mu \mathrm{g} / \mathrm{g}$ in the most enriched of the Cretaceous Pierre Shale samples (Schultz and others, 1980). All available Se data for SDO-1 was obtained by INAA; hydride analysis for Se could be subject to interferences similar to those that affected As analysis by that method. 

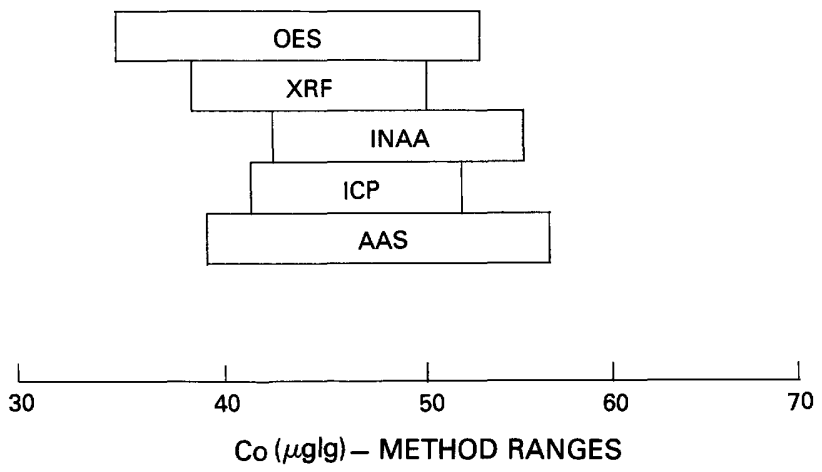

Figure 5. Co data ranges for each method of analysis.

B was reported by relatively few laboratories, and only AES methods using DCP and dc arc excitation were used; thus, adequate confirmation of the interlaboratory averages by an independent technique was impossible. Therefore, despite the precision (rsd of 9 percent) of the intermethod result and an adequate number of laboratories $(n=6)$ reporting B, the B value was not recommended.

Be was similarly determined only by atomic spectrometric techniques; for this element, results obtained by AAS, ICP-AES, and dc arc AES agreed. Confirmation by independent techniques is marginal; all methods are in the same general category, atomic spectrometry. The Be concentration is given as an average but not recommended, based on both an intermethod rsd of 17 percent and the lack of adequate independence of methods.

$\mathrm{Co}$ and $\mathrm{Cr}$ were determined by XRF, INAA, ICP-AES, and dc arc AES with very similar results regardless of method (figs. 5, 6). The same is true of $\mathrm{Ni}$ determined by XRF, ICP-AES, AAS, and dc arc AES (fig. 7). However, $\mathrm{Cu}$ determined by the same methods (fig. 8) shows a distinct difference between the XRF result and the three atomic spectroscopic results. In SDO-1, Cu could be associated with the pyritic fraction of the sample and therefore subject to sampling error within bottle as well as between bottle. This relation would make significant between-bottle variance, which could result in betweenlaboratory and between-method variance, very difficult to identify (Kane, 1992). We observe that the dc arc results are highest, whereas those for XRF, which uses the largest sample weight, are lowest. If sampling were the dominant source of discrepancy between methods, we would expect the reverse, based on sample-size considerations. The largest sample size should provide the highest result, and also the most accurate (Ingamells and Pitard, 1986); therefore, other factors must account for the intermethod discrepancy in $\mathrm{Cu}$ results. Possibly the XRF Cu result is biased due to a matrix effect such as that found by Kopp and Furman (chap. G, this vol.) for several major oxides.

Table 7 summarizes all of the trace element intermethod comparisons derived from the SDO-1 data base.
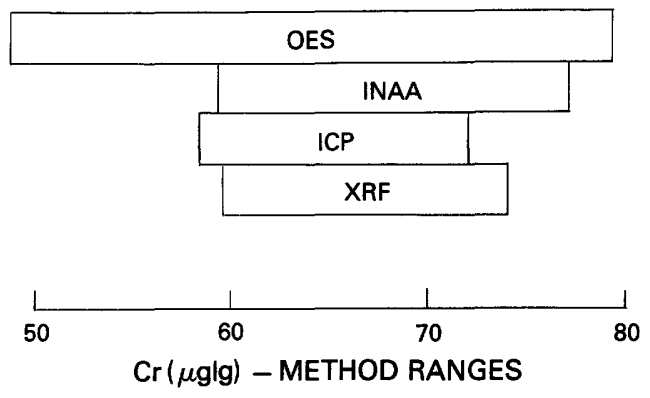

Figure 6. Cr data ranges for each method of analysis.

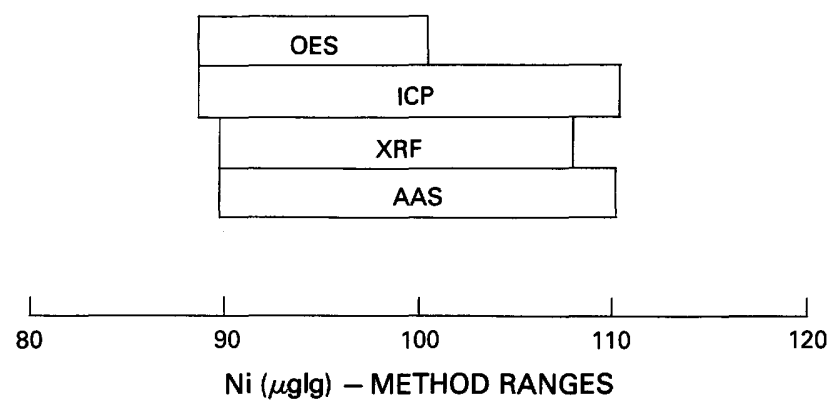

Figure 7. Ni data ranges for each method of analysis.
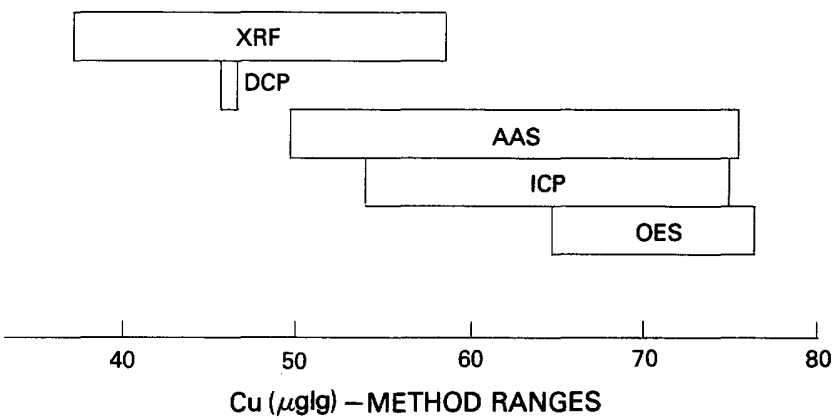

Figure 8. Cu data ranges for each method of analysis.

The reader is referred to the table for conclusions on intermethod agreement for elements not already discussed.

We have already noted that the new methods being used for REE analyses, ICP-AES after separation and ICP-MS, are as accurate as the traditional INAA data for these elements. INAA is used by compilers of referencesample data as the method of choice for a number of other analyses as well. The data in table 7 , and the method-range data plotted in figures 3-8, do not support the superiority of INAA for many of those elements. For example, $\mathrm{Co}$ and $\mathrm{Cr}$ results (figs. 6, 7), discussed above, provide specific illustrations that XRF and ICP-AES both compare very favorably with INAA methods with respect to overall method accuracy and between-laboratory robustness. It is 
Table 7. Between-method analysis of variance for trace elements whose concentrations are recommended

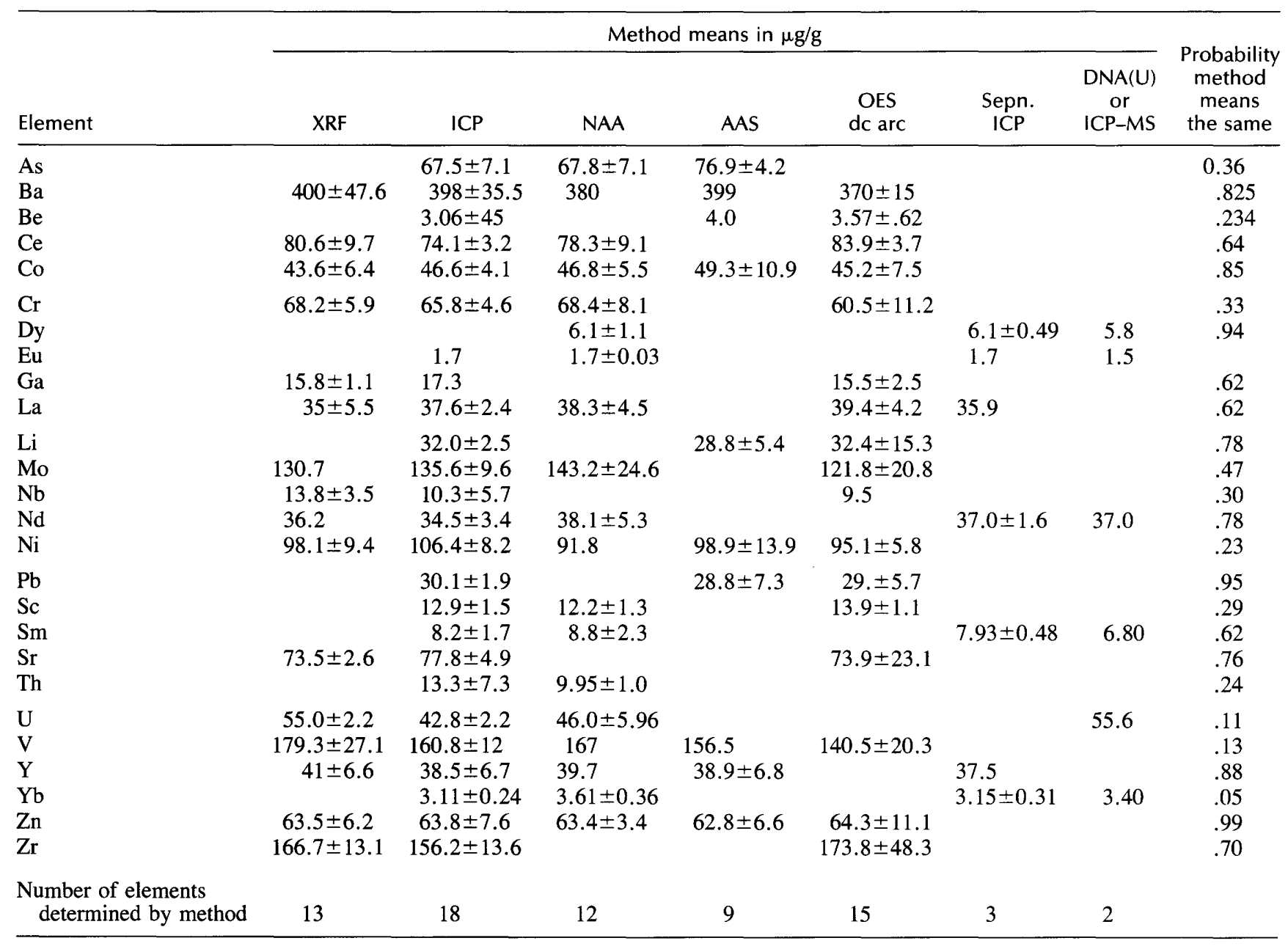

more difficult to comment on comparability of withinlaboratory precision for these methods. In general, where concentrations are high enough and counting times long enough, INAA can be more precise than the other methods, but these conditions are not always possible. Withinlaboratory precision, however, is only one of several figures of merit in determining the general preferability of one analytical method over all others.

\section{UPDATES OF THE ORIGINAL COMPILATION RECOMMENDED CONCENTRATIONS}

Since the time SDO-1 recommended concentrations were first derived (March 1989) for inclusion in the July 1989 Special Issue of Geostandards Newsletter (Govindaraju, 1989), a number of new data contributions have been received and incorporated in the data base. Most of these appeared 1 year later in the full compilation (Kane and others, 1990); additions since then represent less than 1 percent of the total data base. The 1990 recommended concentrations for several rare earth elements differ from the 1989 concentrations (Govindaraju, 1989). Fluorine data received just prior to publication of this volume allowed the fluorine reported in table 1 of the Introduction to be upgraded from range to average. However, revision of most other initial recommended concentrations cannot be justified. It is important that reference-sample recommended concentrations be stable over time for them to be used for instrumental-calibration and method-validation purposes. Some change in a compilation iterated mean must occur any time new data is added to the data base. However, because the changes will have no statistical significance if the newly contributed data value lies within the uncertainty range of the initial recommended concentration, "updating" the recommended concentration based on that change is unwarranted. Several iterations of the compilation average for $\mathrm{SiO}_{2}, \mathrm{Al}_{2} \mathrm{O}_{3}$, and several other constituents at different points during the characterization program illustrate changes that did not justify updating the original recommended concentration (table 8). Changes that did lead to updating of concentrations as initially reported for REE are shown in table 9 . In these instances, intermethod agreement could be established for the updated data but not for the 
Table 8. Samples for which newly added data do not lead to changes in recommended concentrations

\begin{tabular}{lccc}
\hline & $\begin{array}{c}\text { Recommended } \\
\text { based on data } \\
\text { submitted before } \\
\begin{array}{l}\text { Element/ } \\
\text { oxide }\end{array}\end{array}$ & $\begin{array}{c}\text { New average } \\
1 / 89\end{array}$ & $\begin{array}{c}\text { New average } \\
2 / 91\end{array}$ \\
\hline $\begin{array}{l}\text { Major in } \\
\mathrm{SiO}_{2}\end{array}$ & $49.3 \pm 0.63(26)$ & $49.3 \pm 0.61(27)$ & $49.3 \pm 0.61(29)$ \\
$\mathrm{Al}_{2} \mathrm{O}_{3}$ & $12.3 \pm 0.23(22)$ & $2.3 \pm 0.40(27)$ & $2.3 \pm 0.23(28)$ \\
$\mathrm{Fe}_{2} \mathrm{O}_{3}$ & $9.34 \pm 0.21(24)$ & $.36 \pm 0.27(30)$ & $9.38 \pm 0.21(34)$ \\
$T$ & & & \\
$\mathrm{As}$ & $68.5 \pm 8.6(7)$ & $68.2 \pm 8.0(8)$ & $67.6 \pm 9.4(11)$ \\
$\mathrm{Ba}$ & $397 \pm 38(17)$ & $394 \pm 37(19)$ & $394 \pm 36(20)$ \\
$\mathrm{Ce}$ & $79.3 \pm 7.8(18)$ & $78.2 \pm 7.1(23)$ & $78.3 \pm 7.03(24)$ \\
$\mathrm{La}$ & $38.5 \pm 4.4(18)$ & $37.6 \pm 3.0(29)$ & $37.8 \pm 3.1(30)$ \\
$\mathrm{Mo}$ & $134 \pm 21(15)$ & $136 \pm 12(17)$ & $138 \pm 18(19)$ \\
$\mathrm{Zn}$ & $64.1 \pm 6.9(23)$ & $64.5 \pm 7.0(25)$ & $64.6 \pm 7.0(26)$ \\
\hline
\end{tabular}

original (for example, Dy); or the required number of individual laboratory contributions increased enough to meet previously unmet criteria for recommending the concentration, that is, that a minimum of five laboratory results remain after all rejection cycles (for example, Eu).

\section{CONCLUSION}

This study indicates how much information is available in compilation data bases beyond the obvious recommended or average concentrations of individual constituents. Little of that information, however, is typically extracted or applied either to the derivation of recommended concentrations or to uses of the reference samples in calibration, quality control, or other applications. The statistical calculations themselves, having become almost totally automated with the availability of modern computers, are an essential starting point. However, improvement of reference-sample recommended concentrations and thoughtful use of those concentrations in all applications would benefit from a detailed examination of the wealth of information that these data bases provide. Perhaps this discussion will be a first step in that direction.

\section{REFERENCES}

Arnautov, N.V., 1987, Reference samples of chemical composition of natural substances (in Russian): Novosibersk, USSR Academy of Sciences, (see K. Govindaraju, 1989).

Bastenaire, F., 1979, Role of statistics in the certification of standard reference materials: Geostandards Newsletter, v. 3, p. $115-120$.

Cali, J.P., and Reed, W.P., 1974, The role of the National Bureau of Standards standard reference materials in accurate trace analysis in LaFleur, P.D., ed., NBS Special Publication 422, v. 1, p. 41-63.

Coveney, R.M., Jr., and Glascock, M.D., 1989, A review of the origins of metal-rich Pennsylvanian black shales, central USA with a inferred role for basinal brines: Applied Geochemistry, v. 4, p. 347-367.

Deming, W.E., 1943, Statistical adjustment of data: New York, Dover, $18 \mathrm{p}$.

Department of Energy, 1978, Second Eastern Gas Shales Symposium, 1978, Morgantown Energy Technology Center Report METC/SP-78/6, $461 \mathrm{p}$.

Dixon, W.J., and Massey, F.J., Jr., 1983, Introduction to statistical analysis (4th ed.): New York, McGraw-Hill, p. 116-119, 123-124.

Flanagan, F.J., 1976, Descriptions and analyses of eight new USGS rock standards: U.S. Geological Survey Professional Paper 840, 192 pp.

Frost, J.K., Zierath, D.L., and Shimp, N.F., 1985, Chemical composition and geochemistry of the new Albany Shale Group (Devonian-Mississippian) in Illinois: Illinois State Geological Survey Contract/Grant Report 1985-4, 134 pp.

Gladney, E.S., Burns, C.E., and Roelandts, Iwan, 1983, 1982 compilation of elemental concentrations in eleven United States Geological Survey rock standards: Geostandards Newsletter, v. 7, p. 3-226.

Gladney, E.S., and Roelandts, Iwan, 1988, 1987 compilation of elemental concentration data for USGS BHVO-1, MAG-1, QLO-1, RGM-1, SCo-1, SDC-1, SGR-1, and STM-1: Geostandards Newsletter, v. 12, p. 253-362.

Govindaraju, Kuppisami, 1989, 1989 compilation of working values and sample description for 272 geostandards: Geostandards Newsletter, v. 13, Special Issue, 113 pp.

Ingamells, C.O., and Pitard, F.F., 1986, Applied geochemical analysis: New York, Wiley, p. 1-50.

Table 9. Examples for which newly added data result in changes in reported concentrations in $\mu \mathrm{g} / \mathrm{g}$ except as noted

\begin{tabular}{lclclll}
\hline Element & \multicolumn{2}{c}{ Original } & \multicolumn{2}{c}{$\begin{array}{c}\text { Update } \\
\text { Govindaraju, 1989 }\end{array}$} & Kane and others, 1990 & This volume \\
\hline Dy & $5.7 \pm 0.99$ & Avg & $6.0 \pm 0.65$ & Rec & \\
Er & $2.5-4.7$ & Range & $3.6 \pm 0.55$ & Avg & \\
Eu & $1.6 \pm 0.22$ & Avg & $1.6 \pm 0.22$ & Rec & \\
Gd & $6.5 \pm 1.2$ & Avg & $7.4 \pm 1.9$ & Avg & & \\
Ho & $1.1 \pm 3.3$ & Range & $1.2 \pm 0.11$ & Avg & & \\
$\operatorname{Pr}$ & $6-13.8$ & Range & $8.9 \pm 0.66$ & Rec & & \\
$\operatorname{Tm}$ & $0.34-0.56$ & Range & $0.45 \pm 0.08$ & Avg & & \\
$\mathrm{F}(\%)$ & $<0.010-<0.072$ & Range & $<0.010-0.072$ & Range & $0.0697 \pm 0.0089$ & AVG \\
\hline
\end{tabular}


International Organization for Standardization (ISO), 1978, Guide 30 - Terms and definitions used in connection with reference materials, $5 \mathrm{pp}$.

1989, Guide 35-Certification of reference materialsgeneral and statistical principles, $32 \mathrm{pp}$.

Kane, J.S., 1992, Reference samples for use in analytical geochemistry, their availability, preparation, and appropriate use. Journal of Geochemical Exploration, v. 44, p. 37-63.

Kane, J.S., Arbogast, B.F., and Leventhal, J.S., 1990, Characterization of Devonian Ohio Shale SDO-1 as a USGS geochemical reference sample: Geostandards Newsletter, v. 14, p. 169-196.

Kepferle, R.C., de Witt, Wallace, Jr., Flanagan, F.J., 1985, Ohio shale (Devonian), SDO-1, from Rowan County, Kentucky: U.S. Geological Survey Open-File Report 85-145, 14 p.

Lechler, P.J., and Desilets, M.O., 1987, A review of the use of loss on ignition as a measure of total volatiles in whole rock analysis: Chemical Geology, v. 63, p. 341-344.

Leventhal, J.S., Crock, J.G., Mountjoy, Wayne, Thomas, J.A., Shale, V.E., Briggs, P.H., Wahlberg, J.S., and Malcolm, M.J., 1978, Preliminary analytical results for a new U.S. Geological Survey Devonian Ohio Shale standard SDO-1: U.S. Geological Survey Open-File Report 78-447, 11 p.

Leventhal, J.S., and Hosterman, J.W., 1982, Chemical and mineralogical analysis of Devonian black shale samples from Martin County, Kentucky; Carroll and Washington Counties, Ohio; Wise County, Virginia; and Overton County, Tennessee, USA: Chemical Geology, v. 37, p. 239-264.
Leventhal, J.S., and Knapp, T.A., 1988, Preliminary microexamination of polished thin sections of Devonian black shales from the Appalachian Basin by optical microscopy, electron micro-probe and fission track methods, in Lasave, J., Gabriel, Z., eds., Proceedings of the IGCP 254 Inaugural Meeting, October 1987 in Jesenik, Czechoslovakia: Geological Survey, Prague, p. 57-65.

Lister, Brian, 1982, Evaluation of analytical data-A practical guide for geoanalysts: Geostandards Newsletter, v. 6, p. 175-205.

Michaelis, R.E., 1975, The SRM story at NBS in NBS Special Publication 408, p. 237-242.

Natrella, M.G., 1963, Experimental statistics: National Bureau of Standards Handbook 91.

Schultz, L.G., Tourtelot, H.A., Gill, J.R., and Boerngen, J.G., 1980, Composition and properties of the Pierre shale and equivalent rocks, Northern Great Plains region: Geological Survey Professional Paper 1064-B, p. B1-B2.

Shapiro, Leonard, and Brannock, W.W., 1962, Rapid analysis of silicate, carbonate, and phosphate rocks: U.S. Geological Survey Bulletin 1144-A, 56 pp.

Siegel, Sidney, 1956, Nonparametric statistics for the behavioral sciences: New York, McGraw-Hill, p. 47-52.

Uriano, G.A., and Gravatt, C.C., 1977, The role of reference materials and reference methods in chemical analysis: CRC Critical Review in Analytical Chemistry, v. 6, p. 361-441.

Youden, W.J., 1960, The sample, the procedure, and the laboratory: Analytical Chemistry, v. 32, p. 23A-37A. 


\section{APPENDIX 1. MAJOR/MINOR OXIDES: LABORATORY METHODS AND AVERAGES}

\begin{tabular}{|c|c|}
\hline \multicolumn{2}{|l|}{$\mathrm{SiO}_{2}$ data } \\
\hline $\begin{array}{l}\text { Lab code/ } \\
\text { method }\end{array}$ & $\begin{array}{l}\text { Weight percent } \\
\text { in sample }\end{array}$ \\
\hline 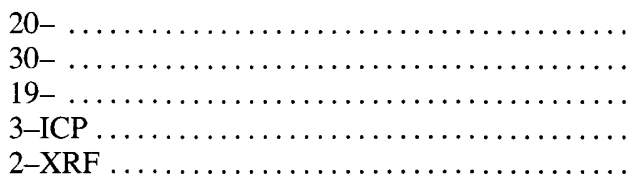 & $\begin{array}{l}23.00 \\
30.72 \\
48.10 \\
48.29 \\
48.43\end{array}$ \\
\hline 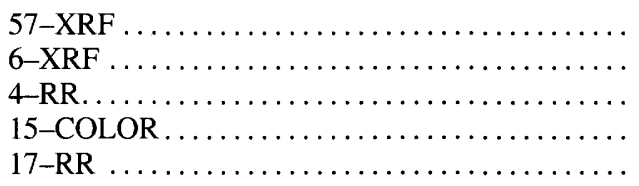 & $\begin{array}{l}48.50 \\
48.57 \\
48.67 \\
48.73 \\
48.77\end{array}$ \\
\hline 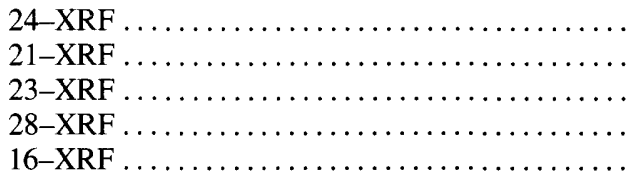 & $\begin{array}{l}48.86 \\
48.94 \\
49.00 \\
49.00 \\
49.15\end{array}$ \\
\hline 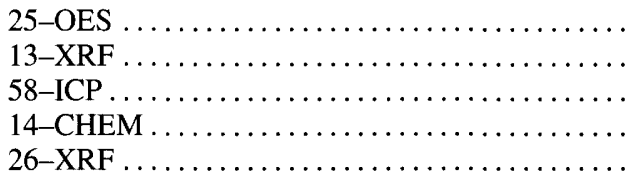 & $\begin{array}{l}49.22 \\
49.24 \\
49.28 \\
49.45 \\
49.56\end{array}$ \\
\hline 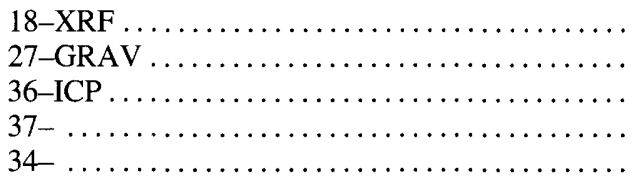 & $\begin{array}{l}49.56 \\
49.90 \\
49.90 \\
49.90 \\
49.90\end{array}$ \\
\hline 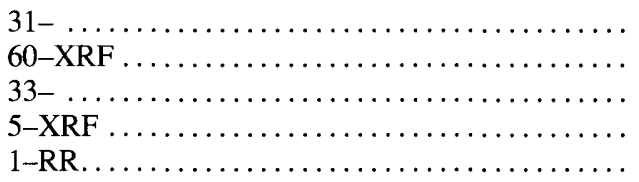 & $\begin{array}{l}49.91 \\
49.91 \\
49.95 \\
50.05 \\
50.05\end{array}$ \\
\hline 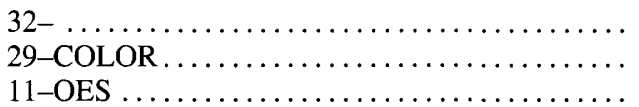 & $\begin{array}{l}50.06 \\
50.50 \\
54.53\end{array}$ \\
\hline
\end{tabular}

\begin{tabular}{|c|c|}
\hline \multicolumn{2}{|l|}{$\mathrm{Al}_{2} \mathrm{O}_{3}$ data } \\
\hline Lab code/ & $\begin{array}{l}\text { Weight percent } \\
\text { in sample }\end{array}$ \\
\hline 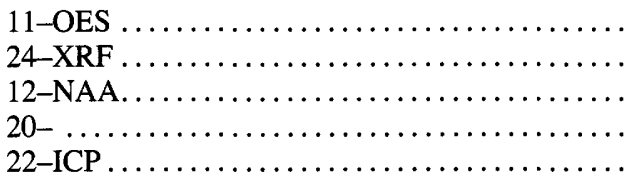 & $\begin{array}{l}11.34 \\
11.65 \\
11.68 \\
11.70 \\
11.88\end{array}$ \\
\hline 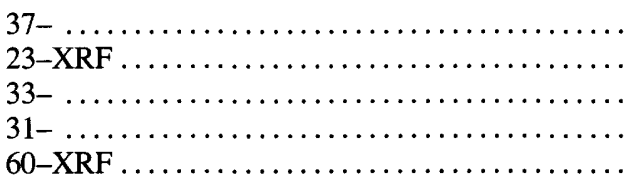 & $\begin{array}{l}12.00 \\
12.00 \\
12.00 \\
12.04 \\
12.04\end{array}$ \\
\hline 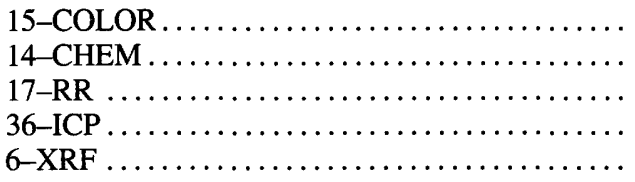 & $\begin{array}{l}12.08 \\
12.08 \\
12.08 \\
12.10 \\
12.10\end{array}$ \\
\hline 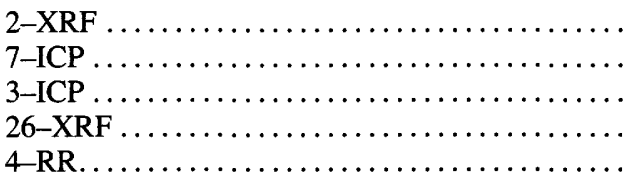 & $\begin{array}{l}12.14 \\
12.29 \\
12.32 \\
12.33 \\
12.34\end{array}$ \\
\hline 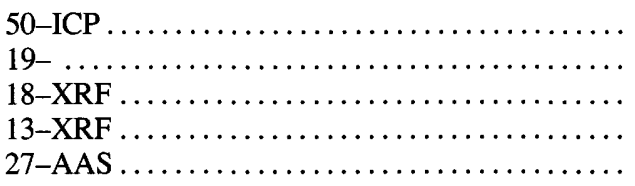 & $\begin{array}{l}12.38 \\
12.38 \\
12.43 \\
12.44 \\
12.50\end{array}$ \\
\hline 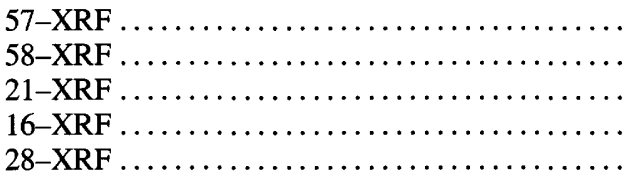 & $\begin{array}{l}12.50 \\
12.50 \\
12.51 \\
12.55 \\
12.60\end{array}$ \\
\hline 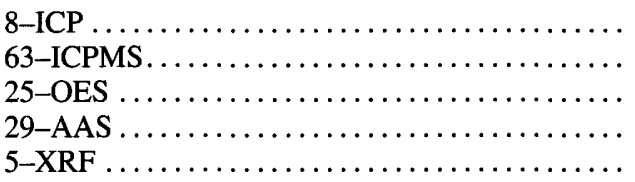 & $\begin{array}{l}12.67 \\
12.67 \\
12.68 \\
12.70 \\
13.02\end{array}$ \\
\hline 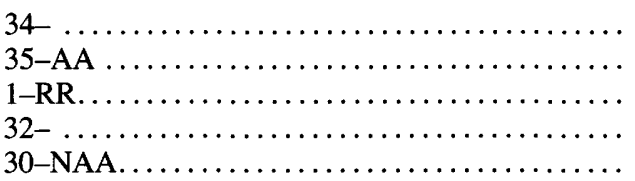 & $\begin{array}{l}13.10 \\
13.10 \\
13.11 \\
14.01 \\
17.45\end{array}$ \\
\hline
\end{tabular}




\section{$\mathrm{Fe}_{2} \mathrm{O}_{3}{ }^{*}$ тот data}

\begin{tabular}{|c|c|}
\hline $\begin{array}{l}\text { Lab code/ } \\
\text { method }\end{array}$ & $\begin{array}{l}\text { Weight percent } \\
\text { in sample }\end{array}$ \\
\hline 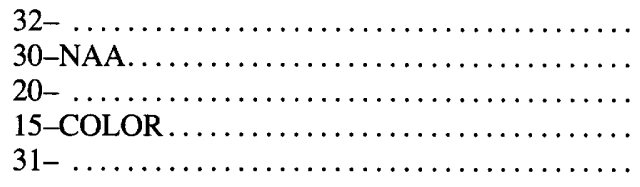 & $\begin{array}{l}0.28 \\
2.31 \\
8.30 \\
8.30 \\
8.60\end{array}$ \\
\hline 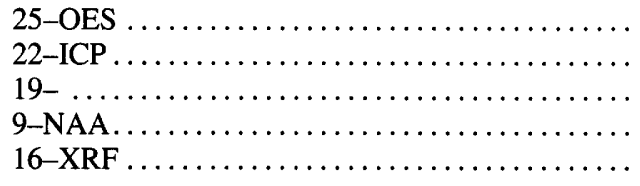 & $\begin{array}{l}8.82 \\
9.01 \\
9.14 \\
9.15 \\
9.15\end{array}$ \\
\hline 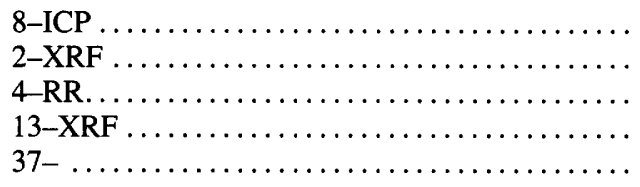 & $\begin{array}{l}9.15 \\
9.16 \\
9.17 \\
9.19 \\
9.22\end{array}$ \\
\hline 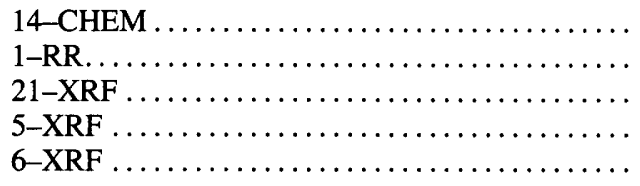 & $\begin{array}{l}9.22 \\
9.34 \\
9.34 \\
9.38 \\
9.41\end{array}$ \\
\hline 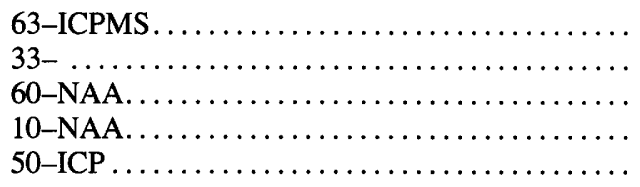 & $\begin{array}{l}9.41 \\
9.43 \\
9.43 \\
9.44 \\
9.45\end{array}$ \\
\hline 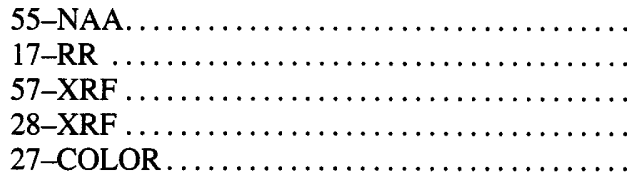 & $\begin{array}{l}9.45 \\
9.49 \\
9.49 \\
9.50 \\
9.54\end{array}$ \\
\hline 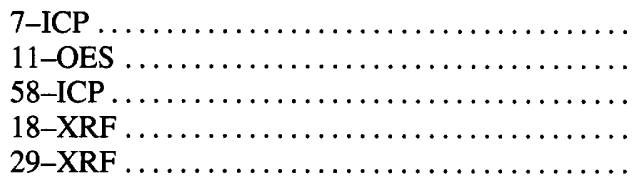 & $\begin{array}{l}9.54 \\
9.54 \\
9.55 \\
9.58 \\
9.60\end{array}$ \\
\hline 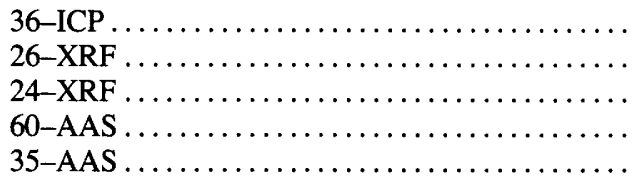 & $\begin{array}{l}9.65 \\
9.66 \\
9.70 \\
9.71 \\
9.75\end{array}$ \\
\hline 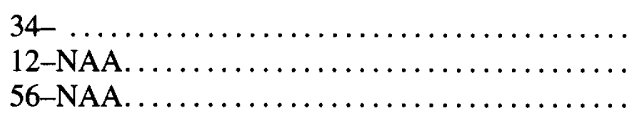 & $\begin{array}{r}9.76 \\
10.12 \\
10.19\end{array}$ \\
\hline
\end{tabular}

* For reporting of total iron only; does not accurately represent iron speciation in sample.

\begin{tabular}{|c|c|}
\hline \multicolumn{2}{|c|}{ FeO data } \\
\hline $\begin{array}{l}\text { Lab code/ } \\
\text { method }\end{array}$ & $\begin{array}{l}\text { Weight percent } \\
\text { in sample }\end{array}$ \\
\hline 24-CHEM & 1.31 \\
\hline 3-CHEM . & 2.25 \\
\hline 19-CHEM & 2.94 \\
\hline 14-CHEM & 5.63 \\
\hline
\end{tabular}

\begin{tabular}{|c|c|}
\hline \multicolumn{2}{|c|}{$\mathrm{Fe}_{2} \mathrm{O}_{3}$ data } \\
\hline $\begin{array}{l}\text { Lab code/ } \\
\text { method }\end{array}$ & $\begin{array}{l}\text { Weight percent } \\
\text { in sample }\end{array}$ \\
\hline 14-CHEM & 3.03 \\
\hline 3-CHEM . & 7.17 \\
\hline 24-CHEM & 8.26 \\
\hline
\end{tabular}

\begin{tabular}{|c|c|}
\hline \multicolumn{2}{|l|}{$\mathrm{CaO}$ data } \\
\hline $\begin{array}{l}\text { Lab code/ } \\
\text { method }\end{array}$ & $\begin{array}{l}\text { Weight percent } \\
\text { in sample }\end{array}$ \\
\hline 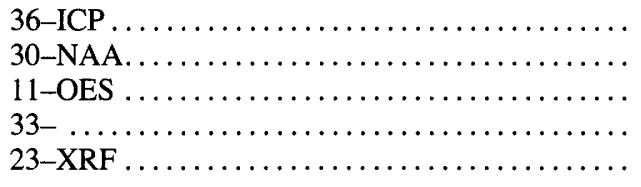 & $\begin{array}{r}0.11 \\
.13 \\
.84 \\
.89 \\
.90\end{array}$ \\
\hline 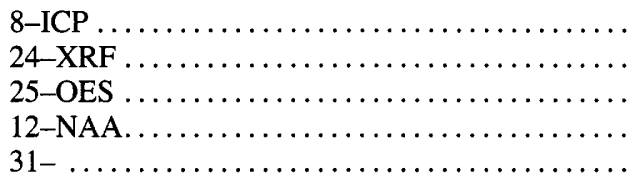 & $\begin{array}{r}.96 \\
.97 \\
.97 \\
1.01 \\
1.01\end{array}$ \\
\hline 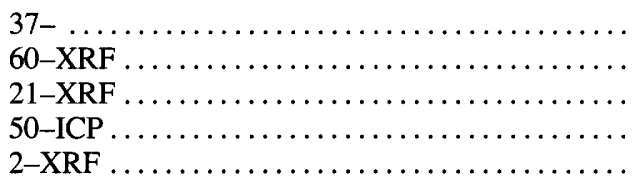 & $\begin{array}{l}1.01 \\
1.01 \\
1.03 \\
1.04 \\
1.05\end{array}$ \\
\hline 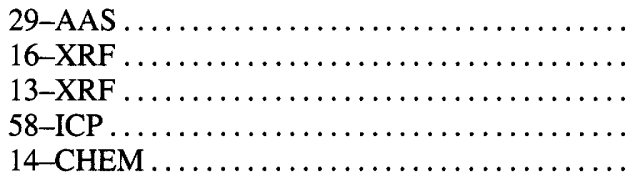 & $\begin{array}{l}1.05 \\
1.05 \\
1.05 \\
1.06 \\
1.06\end{array}$ \\
\hline 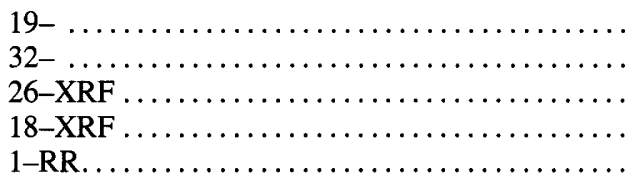 & $\begin{array}{l}1.06 \\
1.06 \\
1.06 \\
1.06 \\
1.06\end{array}$ \\
\hline 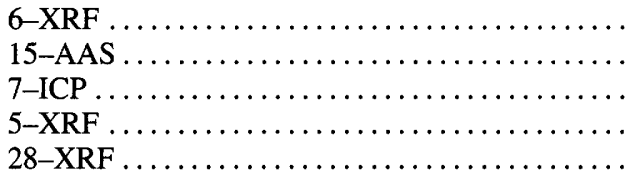 & $\begin{array}{l}1.07 \\
1.08 \\
1.08 \\
1.09 \\
1.10\end{array}$ \\
\hline 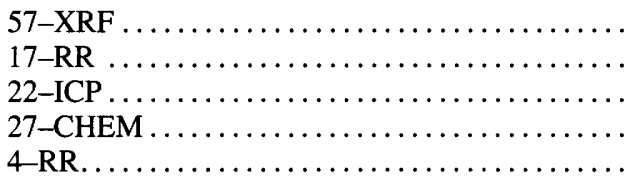 & $\begin{array}{l}1.10 \\
1.11 \\
1.12 \\
1.12 \\
1.13\end{array}$ \\
\hline 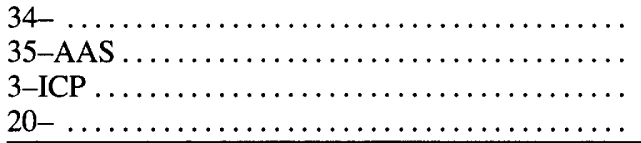 & $\begin{array}{l}1.20 \\
1.20 \\
1.37 \\
1.60\end{array}$ \\
\hline
\end{tabular}




\begin{tabular}{|c|c|}
\hline \multicolumn{2}{|l|}{ MgO data } \\
\hline $\begin{array}{l}\text { Lab code/ } \\
\text { method }\end{array}$ & $\begin{array}{l}\text { Weight percent } \\
\text { in sample }\end{array}$ \\
\hline 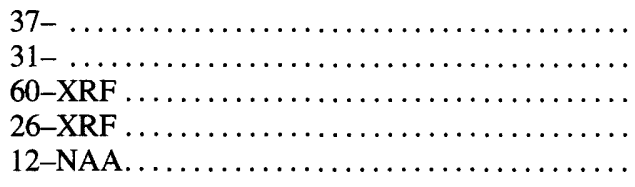 & $\begin{array}{l}1.29 \\
1.29 \\
1.29 \\
1.43 \\
1.45\end{array}$ \\
\hline 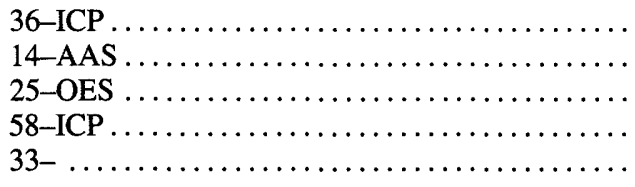 & $\begin{array}{l}1.47 \\
1.48 \\
1.49 \\
1.49 \\
1.51\end{array}$ \\
\hline 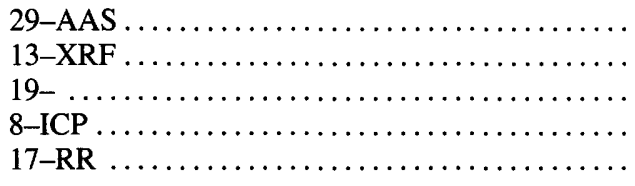 & $\begin{array}{l}1.51 \\
1.52 \\
1.52 \\
1.52 \\
1.53\end{array}$ \\
\hline 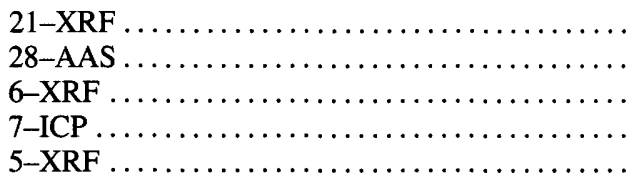 & $\begin{array}{l}1.53 \\
1.53 \\
1.54 \\
1.54 \\
1.54\end{array}$ \\
\hline 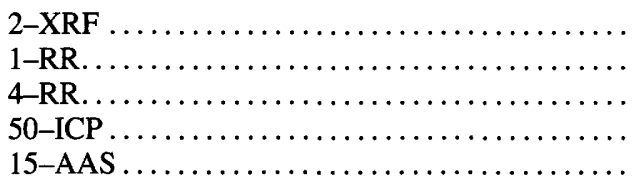 & $\begin{array}{l}1.55 \\
1.55 \\
1.57 \\
1.57 \\
1.57\end{array}$ \\
\hline 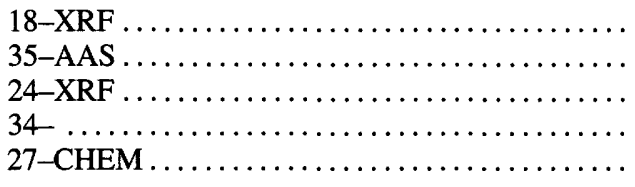 & $\begin{array}{l}1.59 \\
1.59 \\
1.59 \\
1.59 \\
1.59\end{array}$ \\
\hline 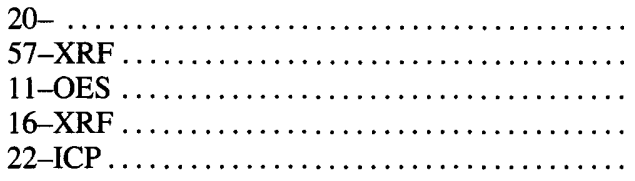 & $\begin{array}{l}1.60 \\
1.60 \\
1.63 \\
1.65 \\
1.66\end{array}$ \\
\hline $\begin{array}{l}3-\mathrm{ICP}{ }^{2} \\
32-\ldots \ldots \ldots \ldots \ldots\end{array}$ & $\begin{array}{l}1.74 \\
1.81\end{array}$ \\
\hline
\end{tabular}

\begin{tabular}{|c|c|}
\hline \multicolumn{2}{|l|}{$\mathrm{Na}_{2} \mathrm{O}$ data } \\
\hline $\begin{array}{l}\text { Lab code/ } \\
\text { method }\end{array}$ & $\begin{array}{l}\text { Weight percent } \\
\text { in sample }\end{array}$ \\
\hline 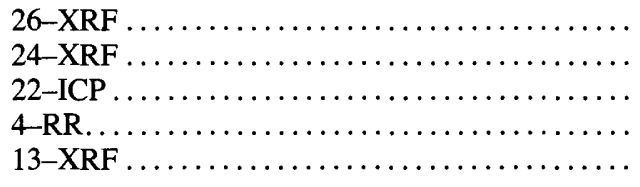 & $\begin{array}{r}0.25 \\
.26 \\
.29 \\
.33 \\
.34\end{array}$ \\
\hline 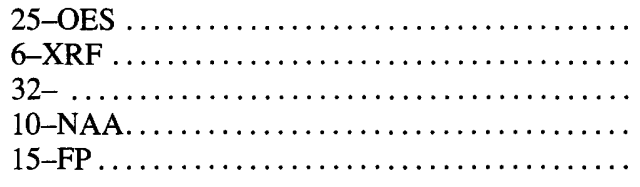 & $\begin{array}{l}.35 \\
.35 \\
.36 \\
.36 \\
.37\end{array}$ \\
\hline 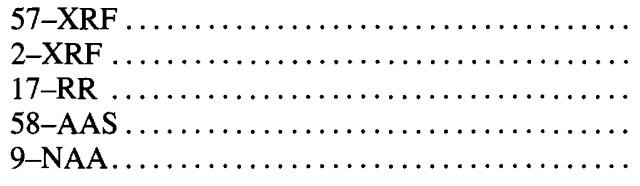 & $\begin{array}{l}.37 \\
.37 \\
.37 \\
.38 \\
.38\end{array}$ \\
\hline 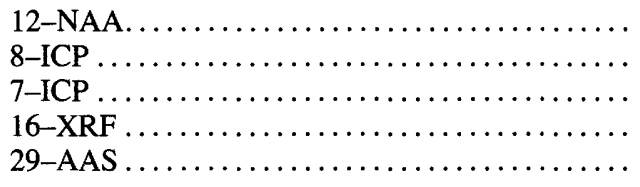 & $\begin{array}{l}.38 \\
.38 \\
.38 \\
.38 \\
.38\end{array}$ \\
\hline 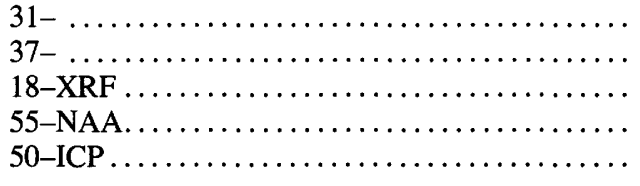 & $\begin{array}{l}.38 \\
.38 \\
.39 \\
.39 \\
.39\end{array}$ \\
\hline 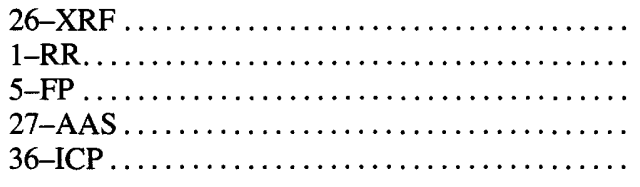 & $\begin{array}{l}.39 \\
.40 \\
.42 \\
.42 \\
.42\end{array}$ \\
\hline 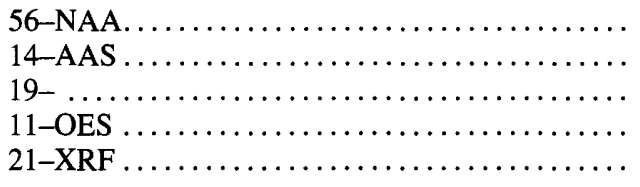 & $\begin{array}{l}.42 \\
.43 \\
.44 \\
.44 \\
.51\end{array}$ \\
\hline 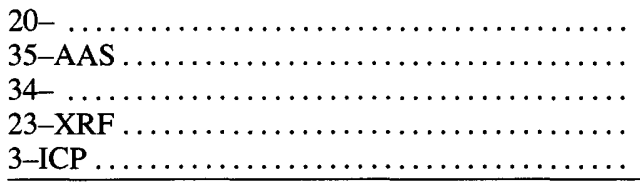 & $\begin{array}{l}.60 \\
.60 \\
.60 \\
.67 \\
.95\end{array}$ \\
\hline
\end{tabular}




\begin{tabular}{|c|c|}
\hline \multicolumn{2}{|l|}{$\mathrm{K}_{2} \mathrm{O}$ data } \\
\hline $\begin{array}{l}\text { Lab code/ } \\
\text { method }\end{array}$ & $\begin{array}{l}\text { Weight percent } \\
\text { in sample }\end{array}$ \\
\hline 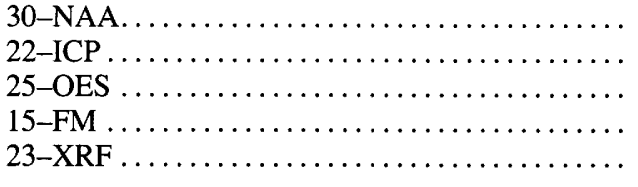 & $\begin{array}{l}2.68 \\
2.79 \\
2.80 \\
2.95 \\
3.00\end{array}$ \\
\hline 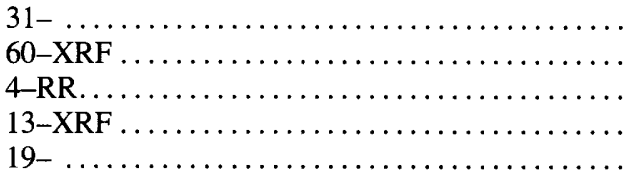 & $\begin{array}{l}3.06 \\
3.06 \\
3.16 \\
3.18 \\
3.20\end{array}$ \\
\hline 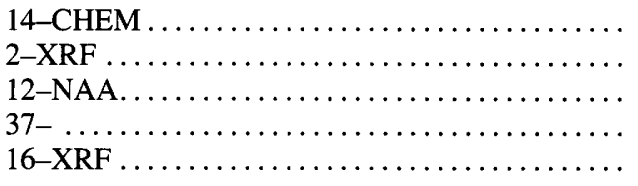 & $\begin{array}{l}3.23 \\
3.24 \\
3.27 \\
3.29 \\
3.29\end{array}$ \\
\hline 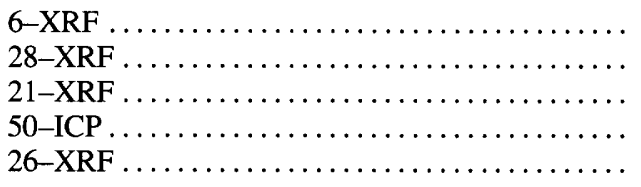 & $\begin{array}{l}3.29 \\
3.30 \\
3.31 \\
3.34 \\
3.35\end{array}$ \\
\hline 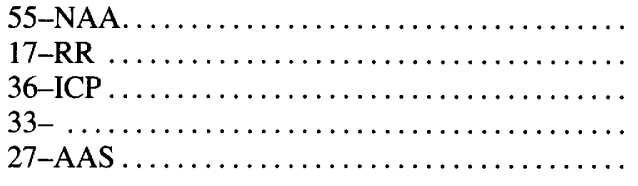 & $\begin{array}{l}3.35 \\
3.36 \\
3.36 \\
3.37 \\
3.37\end{array}$ \\
\hline 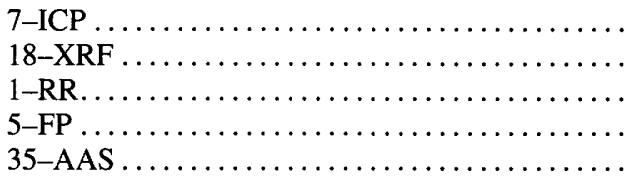 & $\begin{array}{l}3.37 \\
3.37 \\
3.39 \\
3.40 \\
3.41\end{array}$ \\
\hline 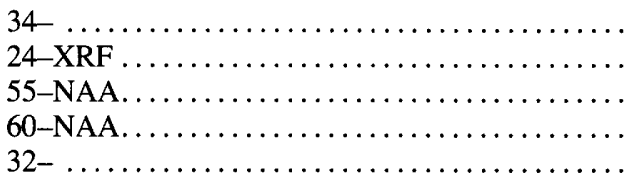 & $\begin{array}{l}3.41 \\
3.43 \\
3.44 \\
3.45 \\
3.46\end{array}$ \\
\hline 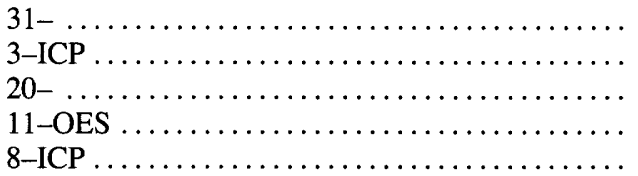 & $\begin{array}{l}3.51 \\
3.60 \\
3.70 \\
3.82 \\
4.60\end{array}$ \\
\hline
\end{tabular}

\begin{tabular}{|c|c|}
\hline \multicolumn{2}{|l|}{$\mathrm{TiO}_{2}$ data } \\
\hline $\begin{array}{l}\text { Lab code/ } \\
\text { method }\end{array}$ & $\begin{array}{l}\text { Weight percent } \\
\text { in sample }\end{array}$ \\
\hline 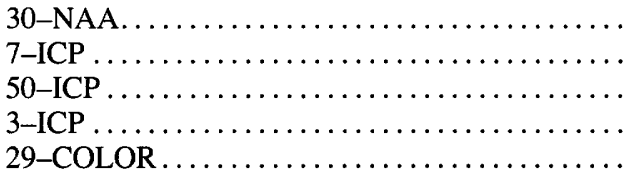 & $\begin{array}{r}0.34 \\
.48 \\
.48 \\
.51 \\
.60\end{array}$ \\
\hline 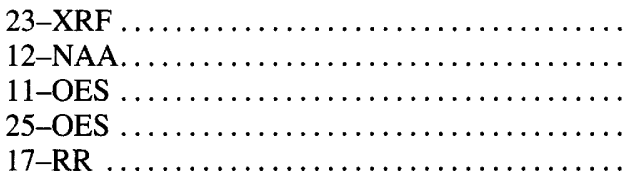 & $\begin{array}{l}.60 \\
.62 \\
.63 \\
.63 \\
.67\end{array}$ \\
\hline 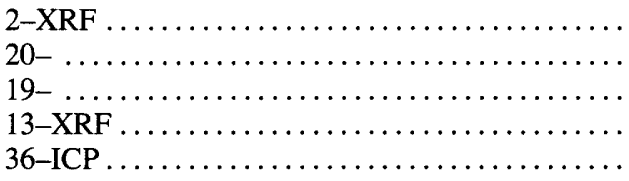 & $\begin{array}{l}.68 \\
.68 \\
.68 \\
.68 \\
.69\end{array}$ \\
\hline 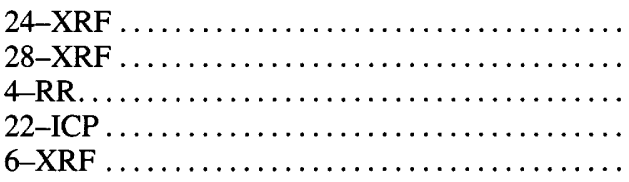 & $\begin{array}{l}.69 \\
.69 \\
.70 \\
.70 \\
.70\end{array}$ \\
\hline 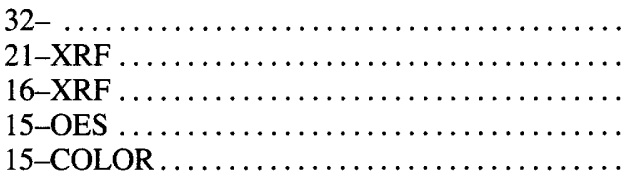 & $\begin{array}{l}.70 \\
.70 \\
.70 \\
.70 \\
.72\end{array}$ \\
\hline 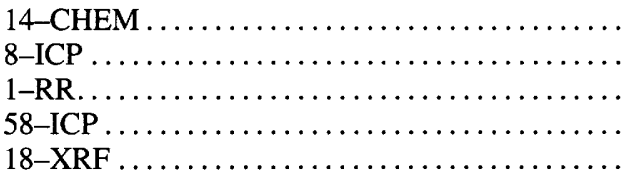 & $\begin{array}{l}.72 \\
.73 \\
.73 \\
.74 \\
.74\end{array}$ \\
\hline 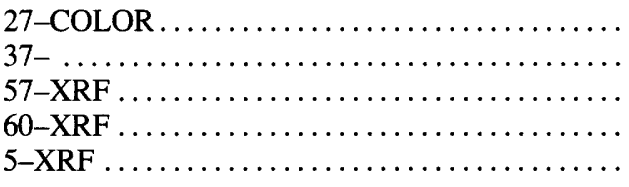 & $\begin{array}{l}.74 \\
.74 \\
.74 \\
.74 \\
.75\end{array}$ \\
\hline 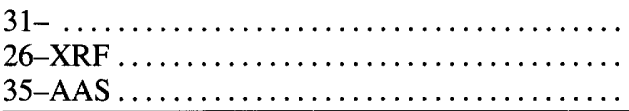 & $\begin{array}{r}.75 \\
.78 \\
1.01\end{array}$ \\
\hline
\end{tabular}




\begin{tabular}{|c|c|}
\hline \multicolumn{2}{|l|}{$\mathrm{P}_{2} \mathrm{O}_{5}$ data } \\
\hline $\begin{array}{l}\text { Lab code/ } \\
\text { method }\end{array}$ & $\begin{array}{l}\text { Weight percent } \\
\text { in sample }\end{array}$ \\
\hline 18-XRF $\ldots \ldots \ldots \ldots \ldots \ldots \ldots \ldots \ldots$ & 0.07 \\
\hline $3-I C P \ldots \ldots \ldots \ldots \ldots \ldots \ldots \ldots \ldots$ & .07 \\
\hline 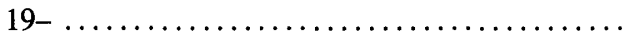 & .08 \\
\hline 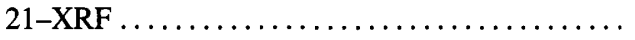 & .09 \\
\hline 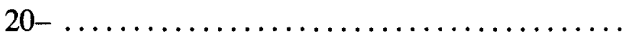 & .10 \\
\hline$\ldots \ldots \ldots \ldots \ldots \ldots \ldots \ldots \ldots \ldots \ldots \ldots$ & .10 \\
\hline $28-$ COLOR $\ldots \ldots \ldots \ldots \ldots \ldots \ldots \ldots \ldots$ & .10 \\
\hline 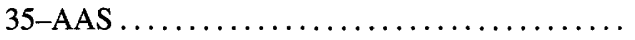 & .10 \\
\hline $37-\ldots \ldots \ldots \ldots \ldots \ldots \ldots \ldots \ldots \ldots \ldots$ & .10 \\
\hline 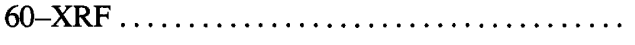 & .10 \\
\hline 14-CHEM $\ldots \ldots \ldots \ldots \ldots \ldots \ldots \ldots \ldots \ldots$ & .10 \\
\hline $5-$ COLOR $\ldots \ldots \ldots \ldots \ldots \ldots \ldots \ldots$ & .11 \\
\hline $15-$ COLOR $\ldots \ldots \ldots \ldots \ldots \ldots \ldots \ldots$ & .11 \\
\hline $27-$ COLOR $\ldots \ldots \ldots \ldots \ldots \ldots \ldots \ldots$ & .11 \\
\hline $13-\mathrm{XRF} \ldots \ldots \ldots \ldots \ldots \ldots \ldots \ldots \ldots$ & .11 \\
\hline $22-I C P \ldots \ldots \ldots \ldots \ldots \ldots \ldots \ldots \ldots \ldots \ldots$ & .11 \\
\hline $33-\ldots \ldots \ldots \ldots \ldots \ldots \ldots \ldots \ldots \ldots$ & .11 \\
\hline $2-\mathrm{XRF} \ldots \ldots \ldots \ldots \ldots \ldots \ldots \ldots \ldots$ & .11 \\
\hline $24-\mathrm{XRF} \ldots \ldots \ldots \ldots \ldots \ldots \ldots \ldots \ldots \ldots$ & .11 \\
\hline $36-I C P \ldots \ldots \ldots \ldots \ldots \ldots \ldots \ldots \ldots \ldots$ & .11 \\
\hline$\ldots \ldots \ldots \ldots \ldots \ldots \ldots \ldots \ldots \ldots$ & .11 \\
\hline $58-C O L O R \ldots \ldots \ldots \ldots \ldots \ldots \ldots \ldots \ldots$ & .11 \\
\hline 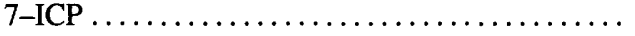 & .12 \\
\hline $29-$ COLOR $\ldots \ldots \ldots \ldots \ldots \ldots \ldots \ldots \ldots \ldots$ & .12 \\
\hline $6-X R F \ldots \ldots \ldots \ldots \ldots \ldots \ldots \ldots \ldots$ & .12 \\
\hline 4RR. & .12 \\
\hline $26-\mathrm{XRF} \ldots \ldots \ldots \ldots \ldots \ldots \ldots \ldots \ldots$ & .12 \\
\hline $57-\mathrm{XRF} \ldots \ldots \ldots \ldots \ldots \ldots \ldots \ldots$ & .12 \\
\hline 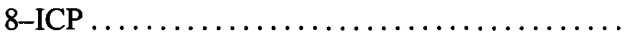 & .13 \\
\hline $1-R R \ldots \ldots \ldots \ldots \ldots \ldots \ldots \ldots \ldots$ & .14 \\
\hline$\ldots \ldots \ldots \ldots \ldots \ldots \ldots$ & .14 \\
\hline$\ldots \ldots \ldots \ldots \ldots$ & .15 \\
\hline 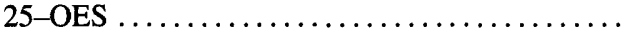 & .24 \\
\hline $11-$ OES $\ldots \ldots \ldots \ldots \ldots \ldots \ldots \ldots \ldots$ & .29 \\
\hline
\end{tabular}

\begin{tabular}{|c|c|}
\hline \multicolumn{2}{|l|}{$\mathrm{MnO}$ data } \\
\hline $\begin{array}{l}\text { Lab code/ } \\
\text { method }\end{array}$ & $\begin{array}{l}\text { Weight percent } \\
\text { in sample }\end{array}$ \\
\hline 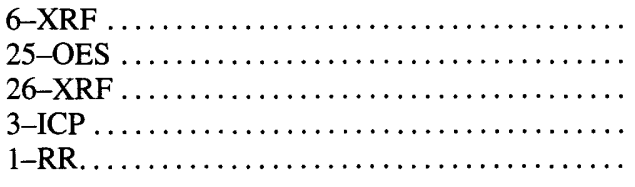 & $\begin{array}{r}0.030 \\
.030 \\
.031 \\
.032 \\
.038\end{array}$ \\
\hline 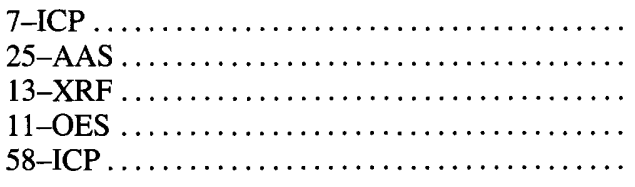 & $\begin{array}{l}.039 \\
.039 \\
.039 \\
.039 \\
.039\end{array}$ \\
\hline 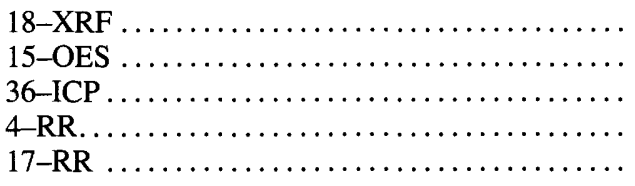 & $\begin{array}{l}.039 \\
.040 \\
.040 \\
.040 \\
.040\end{array}$ \\
\hline 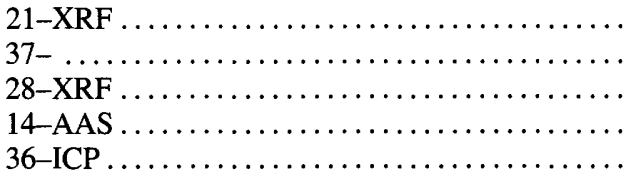 & $\begin{array}{l}.040 \\
.040 \\
.040 \\
.041 \\
.041\end{array}$ \\
\hline 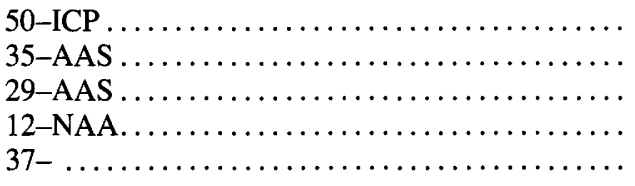 & $\begin{array}{l}.041 \\
.042 \\
.042 \\
.043 \\
.043\end{array}$ \\
\hline 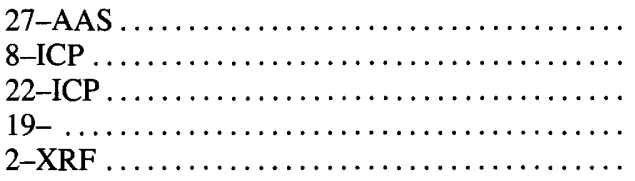 & $\begin{array}{l}.043 \\
.046 \\
.046 \\
.050 \\
.050\end{array}$ \\
\hline $24-X R F \ldots \ldots \ldots \ldots \ldots \ldots \ldots \ldots \ldots \ldots \ldots \ldots$ & .080 \\
\hline
\end{tabular}




\begin{tabular}{|c|c|}
\hline \multicolumn{2}{|l|}{ LOI data } \\
\hline $\begin{array}{l}\text { Lab code/ } \\
\text { method }\end{array}$ & $\begin{array}{l}\text { Weight percent } \\
\text { in sample }\end{array}$ \\
\hline 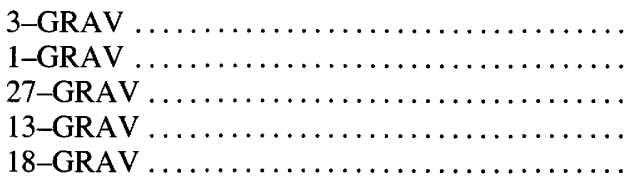 & $\begin{array}{l}19.56 \\
19.56 \\
19.78 \\
20.83 \\
20.91\end{array}$ \\
\hline 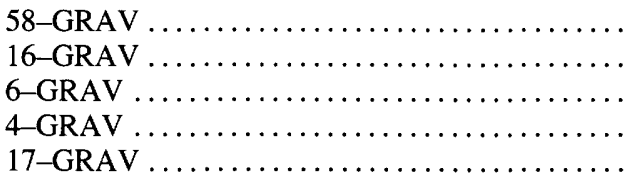 & $\begin{array}{l}21.37 \\
21.38 \\
21.57 \\
21.63 \\
21.82\end{array}$ \\
\hline 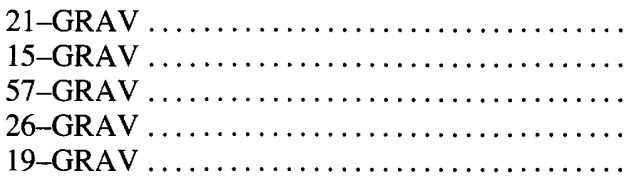 & $\begin{array}{l}21.90 \\
21.93 \\
22.00 \\
22.35 \\
22.37\end{array}$ \\
\hline 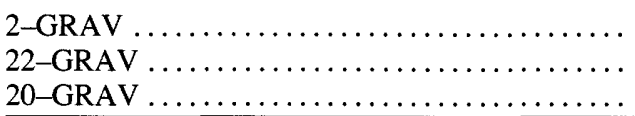 & $\begin{array}{l}22.84 \\
23.08 \\
33.00\end{array}$ \\
\hline
\end{tabular}

\begin{tabular}{|c|c|}
\hline \multicolumn{2}{|l|}{$\mathrm{C}_{\text {TOt }}$ data } \\
\hline $\begin{array}{l}\text { Lab code/ } \\
\text { method }\end{array}$ & $\begin{array}{l}\text { Weight percent } \\
\text { in sample }\end{array}$ \\
\hline 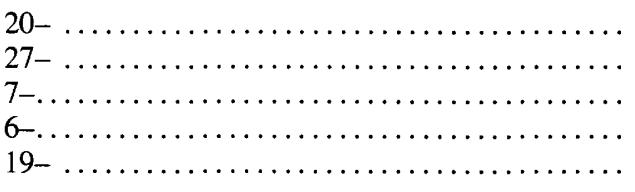 & $\begin{array}{l}8.97 \\
9.27 \\
9.85 \\
9.96 \\
9.96\end{array}$ \\
\hline 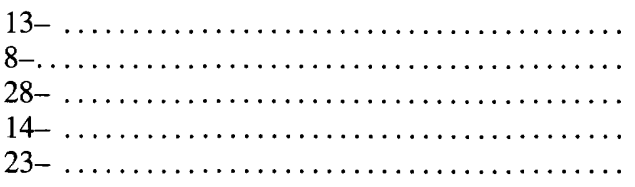 & $\begin{array}{r}9.96 \\
9.98 \\
10.00 \\
10.26 \\
10.36\end{array}$ \\
\hline 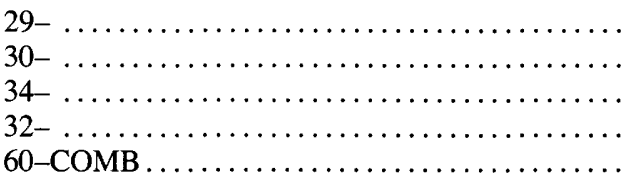 & $\begin{array}{l}10.40 \\
10.40 \\
10.40 \\
10.43 \\
10.53\end{array}$ \\
\hline 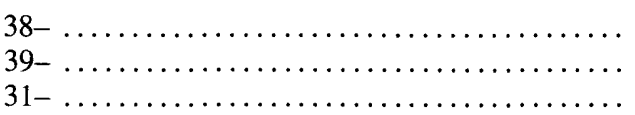 & $\begin{array}{l}10.89 \\
11.80 \\
13.79\end{array}$ \\
\hline
\end{tabular}

\begin{tabular}{|c|c|}
\hline \multicolumn{2}{|l|}{ Hydrogen (percent) } \\
\hline $\begin{array}{l}\text { Lab code/ } \\
\text { rejection method }\end{array}$ & $\begin{array}{l}\text { Weight percent } \\
\text { in sample }\end{array}$ \\
\hline 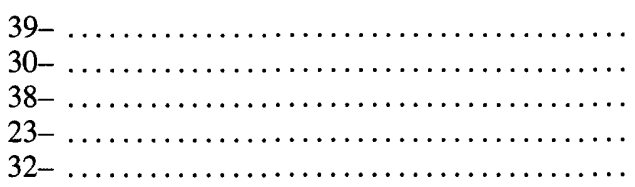 & $\begin{array}{l}1.24 \\
1.30 \\
1.30 \\
1.33 \\
1.34\end{array}$ \\
\hline 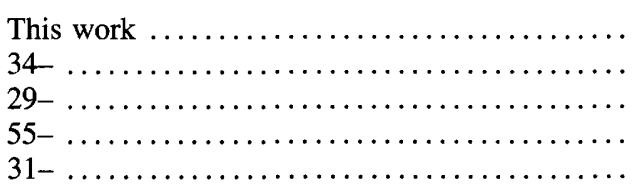 & $\begin{array}{l}1.34 \\
1.35 \\
1.40 \\
1.45 \\
1.83\end{array}$ \\
\hline
\end{tabular}

\begin{tabular}{|c|c|}
\hline \multicolumn{2}{|l|}{ Nitrogen (percent) } \\
\hline $\begin{array}{l}\text { Lab code/ } \\
\text { rejection method }\end{array}$ & $\begin{array}{l}\text { Weight percent } \\
\text { in sample }\end{array}$ \\
\hline $31-$ & 0.31 \\
\hline $38-$ & .31 \\
\hline$\ldots \ldots \ldots \ldots$ & .32 \\
\hline This work $\ldots \ldots \ldots \ldots \ldots \ldots \ldots \ldots \ldots \ldots$ & .33 \\
\hline 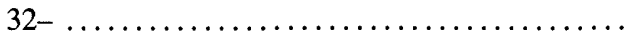 & .34 \\
\hline $23-$ & .40 \\
\hline $30-\ldots$ & .40 \\
\hline $39-\ldots \ldots \ldots \ldots \ldots \ldots \ldots \ldots \ldots \ldots \ldots \ldots$ & 1.21 \\
\hline
\end{tabular}

\begin{tabular}{|c|c|}
\hline \multicolumn{2}{|l|}{ Constitutional water (percent) } \\
\hline $\begin{array}{l}\text { Lab code/ } \\
\text { rejection method }\end{array}$ & $\begin{array}{l}\text { Weight percent } \\
\text { in sample }\end{array}$ \\
\hline Compilation average. & 2.93 \\
\hline $17-\ldots \ldots \ldots \ldots \ldots$ & 4.41 \\
\hline$\ldots \ldots \ldots \ldots \ldots \ldots \ldots \ldots$ & 7.12 \\
\hline
\end{tabular}


APPENDIX 2.

TRACE ELEMENTS: LABORATORY METHODS AND AVERAGES

\begin{tabular}{|c|c|}
\hline \multicolumn{2}{|l|}{ Ag data } \\
\hline $\begin{array}{l}\text { Lab code/ } \\
\text { method }\end{array}$ & $\begin{array}{c}\mu \mathrm{g} / \mathrm{g} \\
\text { in sample }\end{array}$ \\
\hline 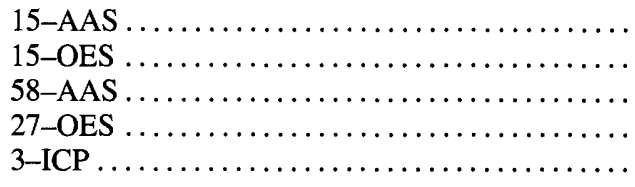 & $\begin{array}{l}0.0944 \\
\quad .1650 \\
<.40 \\
<.40 \\
<.50\end{array}$ \\
\hline 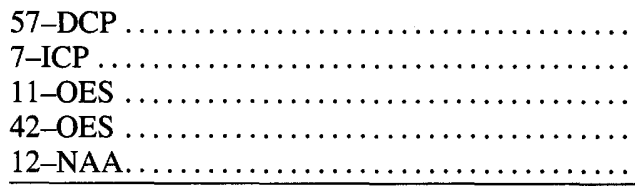 & $\begin{array}{l}<.50 \\
<2 \\
<2 \\
<3 \\
<4\end{array}$ \\
\hline
\end{tabular}

\begin{tabular}{|c|c|}
\hline \multicolumn{2}{|l|}{ B data } \\
\hline $\begin{array}{l}\text { Lab code/ } \\
\text { method }\end{array}$ & $\begin{array}{c}\mu \mathrm{g} / \mathrm{g} \\
\text { in sample }\end{array}$ \\
\hline 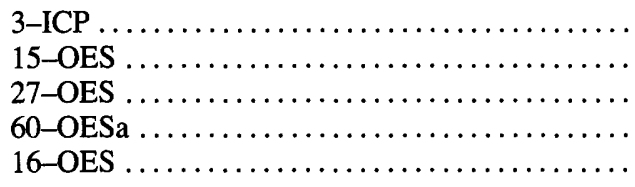 & $\begin{array}{l}92.5 \\
120 \\
120 \\
120 \\
129\end{array}$ \\
\hline 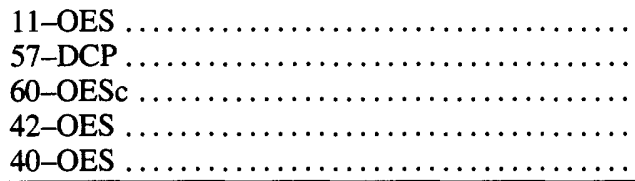 & $\begin{array}{l}130 \\
130 \\
140 \\
145 \\
170\end{array}$ \\
\hline
\end{tabular}

\begin{tabular}{|c|c|}
\hline \multicolumn{2}{|l|}{ As data } \\
\hline $\begin{array}{l}\text { Lab code/ } \\
\text { method }\end{array}$ & $\begin{array}{c}\mu \mathrm{g} / \mathrm{g} \\
\text { in sample }\end{array}$ \\
\hline 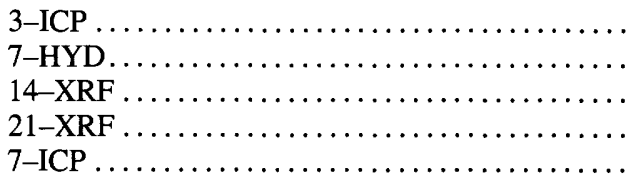 & $\begin{array}{c}<10.0 \\
38 \\
50.3 \\
55.0 \\
62.5\end{array}$ \\
\hline 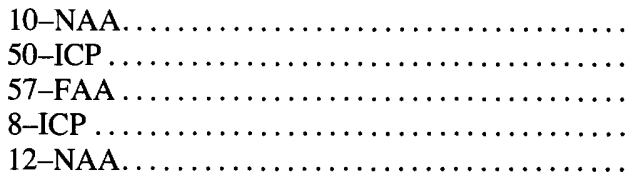 & $\begin{array}{l}62.7 \\
66.1 \\
69.0 \\
72.5 \\
72.8\end{array}$ \\
\hline 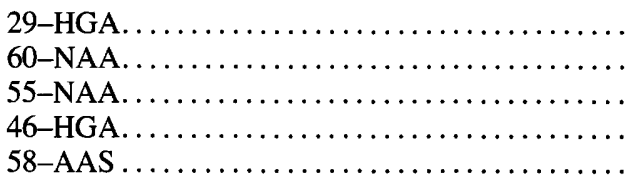 & $\begin{array}{l}74.0 \\
79 \\
79.0 \\
79.8 \\
100\end{array}$ \\
\hline $23-{ }^{2}-{ }_{1} A \ldots \ldots \ldots \ldots \ldots \ldots \ldots \ldots$ & 104 \\
\hline
\end{tabular}

\begin{tabular}{|c|c|}
\hline \multicolumn{2}{|l|}{ Au data } \\
\hline $\begin{array}{l}\text { Lab code/ } \\
\text { method }\end{array}$ & $\begin{array}{c}\mu g / g \\
\text { in sample }\end{array}$ \\
\hline 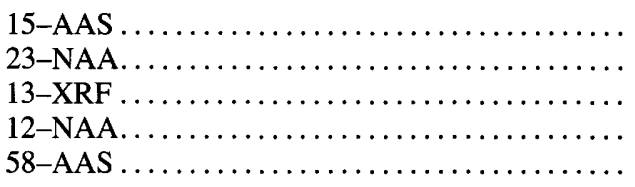 & $\begin{array}{r}0.0014 \\
.0020 \\
.0035 \\
<.010 \\
<.10\end{array}$ \\
\hline $\begin{array}{l}57-\mathrm{DCP}, \ldots \ldots \ldots \ldots \ldots \ldots \ldots \ldots \ldots \ldots \ldots \ldots \ldots \\
7-\mathrm{ICP} \ldots \ldots \ldots \ldots \ldots \ldots \ldots \ldots \ldots \ldots\end{array}$ & $\begin{array}{l}<2 \\
<8\end{array}$ \\
\hline
\end{tabular}

\begin{tabular}{|c|c|}
\hline \multicolumn{2}{|l|}{ Ba data } \\
\hline $\begin{array}{l}\text { Lab code/ } \\
\text { method }\end{array}$ & $\begin{array}{c}\mu \mathrm{g} / \mathrm{g} \\
\text { in sample }\end{array}$ \\
\hline 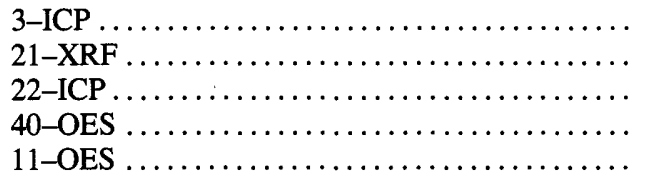 & $\begin{array}{l}211 \\
318 \\
335 \\
360 \\
363\end{array}$ \\
\hline 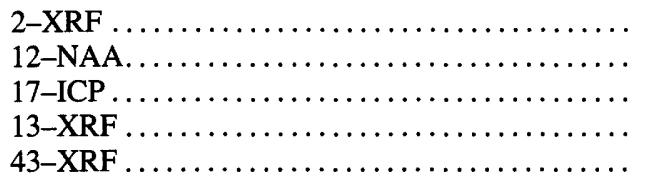 & $\begin{array}{l}369 \\
380 \\
381 \\
385 \\
388\end{array}$ \\
\hline 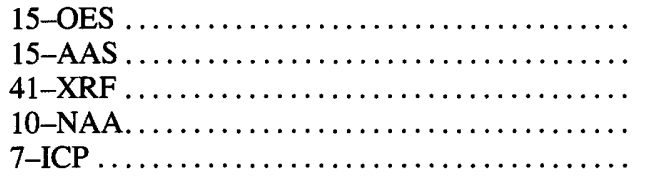 & $\begin{array}{l}390 \\
398 \\
403 \\
403 \\
407\end{array}$ \\
\hline 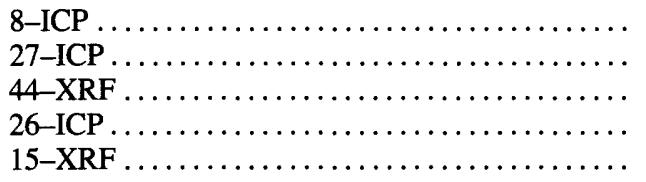 & $\begin{array}{l}415 \\
420 \\
424 \\
431 \\
444\end{array}$ \\
\hline 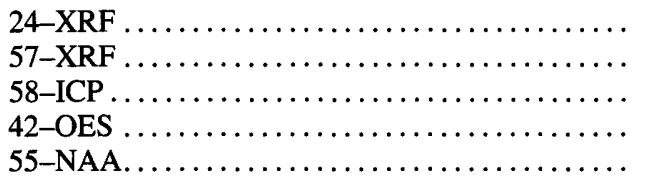 & $\begin{array}{l}473 \\
481 \\
495 \\
529 \\
550\end{array}$ \\
\hline 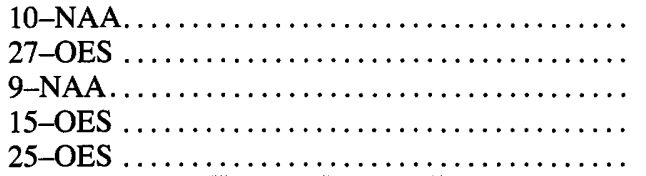 & $\begin{array}{l}598 \\
600 \\
633 \\
640 \\
743\end{array}$ \\
\hline
\end{tabular}




\begin{tabular}{|c|c|}
\hline \multicolumn{2}{|l|}{ Be data } \\
\hline $\begin{array}{l}\text { Lab code/ } \\
\text { method }\end{array}$ & $\begin{array}{c}\mu \mathrm{g} / \mathrm{g} \\
\text { in sample }\end{array}$ \\
\hline 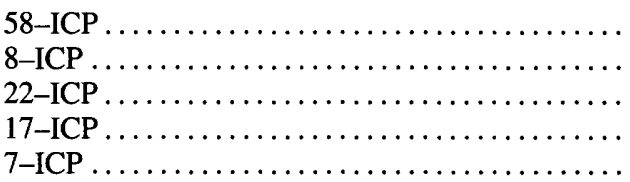 & $\begin{array}{l}2.00 \\
2.75 \\
2.78 \\
2.93 \\
3.00\end{array}$ \\
\hline 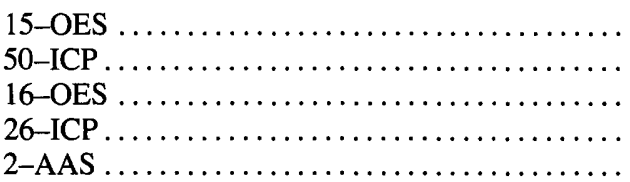 & $\begin{array}{l}3.01 \\
3.09 \\
3.48 \\
3.85 \\
4.0\end{array}$ \\
\hline 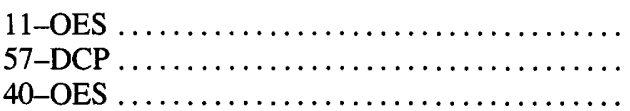 & $\begin{array}{l}4.25 \\
5.00 \\
5.83\end{array}$ \\
\hline
\end{tabular}

\begin{tabular}{|c|c|}
\hline \multicolumn{2}{|l|}{ Ce data } \\
\hline $\begin{array}{l}\text { Lab code/ } \\
\text { method }\end{array}$ & $\begin{array}{c}\mu \mathrm{g} / \mathrm{g} \\
\text { in sample }\end{array}$ \\
\hline 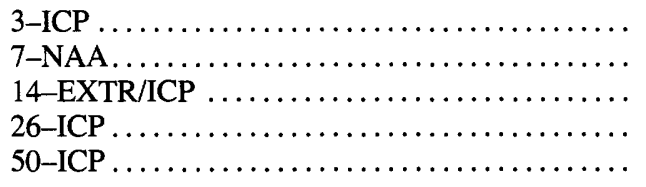 & $\begin{array}{l}63.0 \\
64.0 \\
65.0 \\
68.0 \\
68.7\end{array}$ \\
\hline 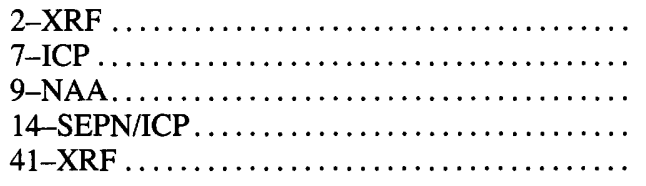 & $\begin{array}{l}70.0 \\
70.5 \\
70.8 \\
71.3 \\
71.8\end{array}$ \\
\hline 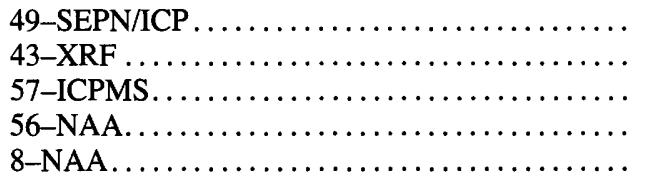 & $\begin{array}{l}71.8 \\
72.3 \\
73.2 \\
73.7 \\
74.2\end{array}$ \\
\hline 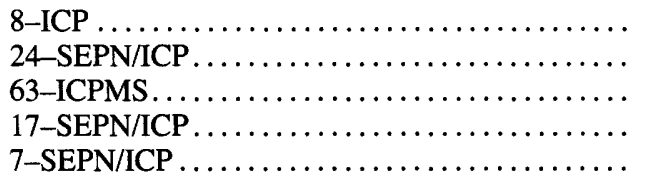 & $\begin{array}{l}75.0 \\
76.2 \\
76.2 \\
76.7 \\
76.8\end{array}$ \\
\hline 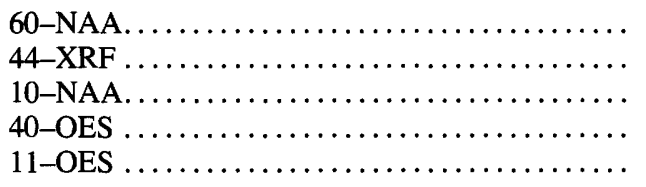 & $\begin{array}{l}77.4 \\
78.5 \\
78.5 \\
80.2 \\
84.0\end{array}$ \\
\hline 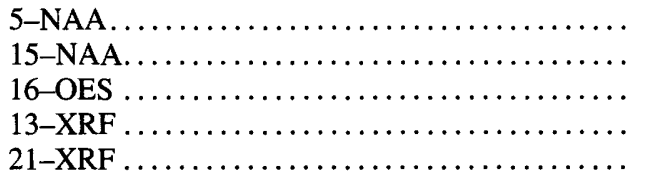 & $\begin{array}{l}85.0 \\
87.3 \\
87.5 \\
89.7 \\
90.0\end{array}$ \\
\hline 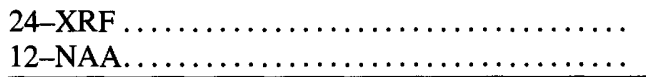 & $\begin{array}{l}92.0 \\
100\end{array}$ \\
\hline
\end{tabular}

\begin{tabular}{|c|c|}
\hline \multicolumn{2}{|l|}{ Bi data } \\
\hline $\begin{array}{l}\text { Lab code/ } \\
\text { method }\end{array}$ & $\begin{array}{c}\mu g / g \\
\text { in sample }\end{array}$ \\
\hline $57-D C P \ldots \ldots \ldots \ldots$ & 0.2 \\
\hline 2-XRF .. & $<2$ \\
\hline $3-\mathrm{ICP} \ldots$ & $<10$ \\
\hline$\ldots \ldots \ldots \ldots \ldots \ldots \ldots \ldots$ & $<10$ \\
\hline $21-\mathrm{XRF} \ldots \ldots \ldots \ldots \ldots \ldots \ldots \ldots \ldots \ldots \ldots$ & $<30$ \\
\hline
\end{tabular}

\begin{tabular}{|c|c|}
\hline \multicolumn{2}{|c|}{ Br data } \\
\hline $\begin{array}{l}\text { Lab code/ } \\
\text { method }\end{array}$ & $\begin{array}{c}\mu \mathrm{g} / \mathrm{g} \\
\text { in sample }\end{array}$ \\
\hline $\begin{array}{l}\text { 12-NAA. } \\
60 \text {-NAA. }\end{array}$ & $\begin{array}{l}5 \\
3.6\end{array}$ \\
\hline
\end{tabular}

\begin{tabular}{|c|c|}
\hline \multicolumn{2}{|l|}{ Cd data } \\
\hline $\begin{array}{l}\text { Lab code/ } \\
\text { method }\end{array}$ & $\begin{array}{c}\mu \mathrm{g} / \mathrm{g} \\
\text { in sample }\end{array}$ \\
\hline 57-DCP . & $<0.2$ \\
\hline$\ldots \ldots \ldots \ldots \ldots \ldots$ & $<.5$ \\
\hline $58-\mathrm{ICP} \ldots \ldots \ldots \ldots \ldots \ldots \ldots \ldots \ldots \ldots \ldots \ldots \ldots \ldots \ldots$ & 1 \\
\hline $7-\mathrm{ICP} \ldots \ldots \ldots \ldots \ldots \ldots$ & $<2$ \\
\hline $11-$ OES $\ldots \ldots \ldots \ldots$ & $<3$ \\
\hline 27-ICP.. & 7 \\
\hline $21-\mathrm{XRF} \ldots \ldots \ldots \ldots \ldots \ldots \ldots \ldots \ldots \ldots \ldots \ldots \ldots$ & $<20$ \\
\hline
\end{tabular}

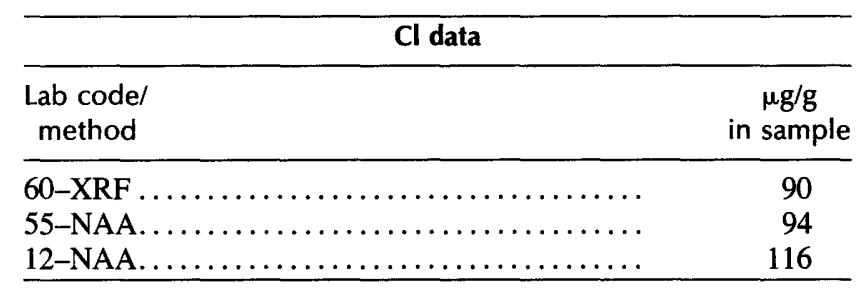




\begin{tabular}{|c|c|}
\hline \multicolumn{2}{|l|}{ Co data } \\
\hline $\begin{array}{l}\text { Lab code/ } \\
\text { method }\end{array}$ & $\begin{array}{c}\mu \mathrm{g} / \mathrm{g} \\
\text { in sample }\end{array}$ \\
\hline 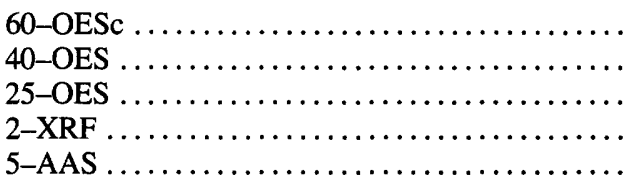 & $\begin{array}{l}35 \\
36.5 \\
37.5 \\
39.8 \\
40.0\end{array}$ \\
\hline 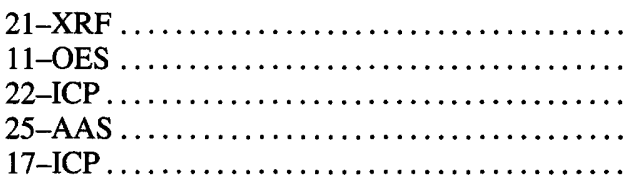 & $\begin{array}{l}40.0 \\
40.3 \\
41.8 \\
42.0 \\
42.5\end{array}$ \\
\hline 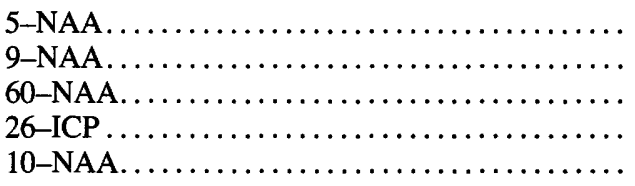 & $\begin{array}{l}43.1 \\
43.5 \\
44 \\
44.0 \\
44.4\end{array}$ \\
\hline 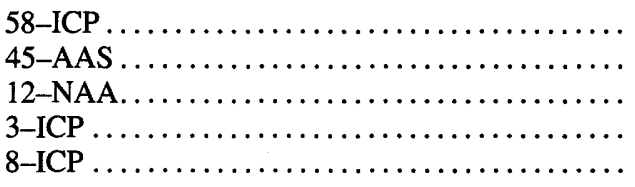 & $\begin{array}{l}44.5 \\
46.5 \\
46.8 \\
47.0 \\
48.0\end{array}$ \\
\hline 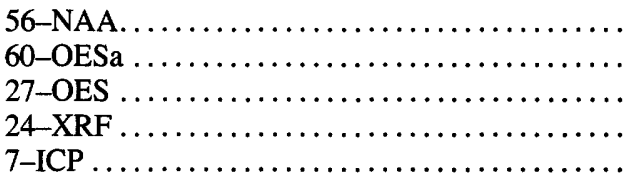 & $\begin{array}{l}48.9 \\
49 \\
50.0 \\
51.0 \\
51.3\end{array}$ \\
\hline 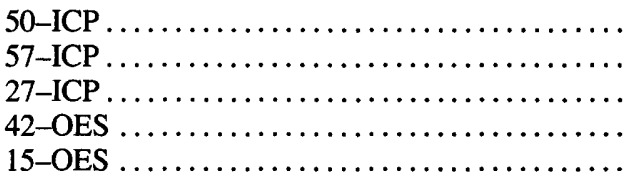 & $\begin{array}{l}51.4 \\
52.0 \\
52.0 \\
53.0 \\
53.0\end{array}$ \\
\hline 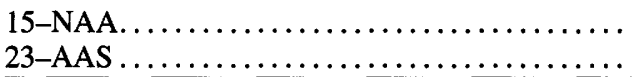 & $\begin{array}{l}56.3 \\
57.0\end{array}$ \\
\hline
\end{tabular}

\begin{tabular}{|c|c|}
\hline \multicolumn{2}{|l|}{ Cr data } \\
\hline $\begin{array}{l}\text { Lab code/ } \\
\text { method }\end{array}$ & $\begin{array}{c}\mu \mathrm{g} / \mathrm{g} \\
\text { in sample }\end{array}$ \\
\hline 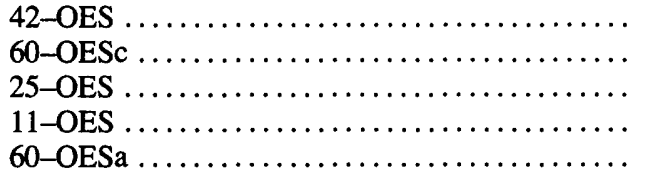 & $\begin{array}{l}47.8 \\
50 \\
51.5 \\
55.8 \\
56\end{array}$ \\
\hline
\end{tabular}

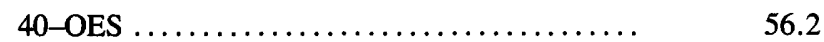

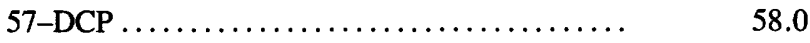

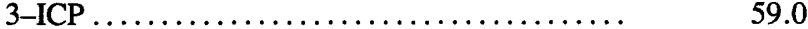

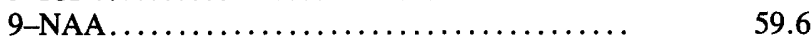

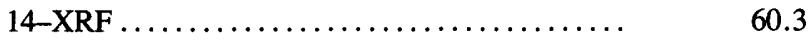

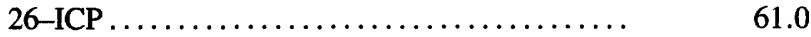

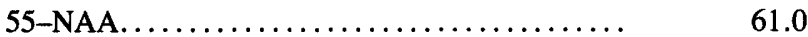

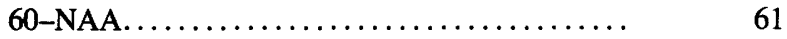

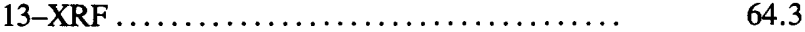

$10-N A A \ldots \ldots \ldots \ldots \ldots \ldots \ldots \ldots \ldots \ldots \ldots \ldots \ldots, 64.5$

$58-\mathrm{ICP} \ldots \ldots \ldots \ldots \ldots \ldots \ldots \ldots \ldots \ldots \ldots \ldots \ldots$

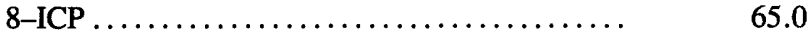

$41-\mathrm{XRF} \ldots \ldots \ldots \ldots \ldots \ldots \ldots \ldots \ldots \ldots \ldots \ldots \ldots$

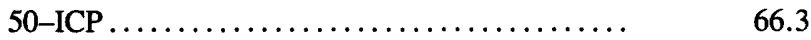

$27-\mathrm{ICP} \ldots \ldots \ldots \ldots \ldots \ldots \ldots \ldots \ldots \ldots \ldots \ldots \ldots$

$22-\mathrm{ICP} \ldots \ldots \ldots \ldots \ldots \ldots \ldots \ldots \ldots \ldots \ldots \ldots \ldots . \ldots \ldots$

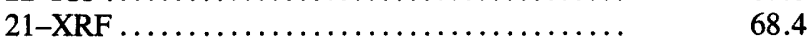

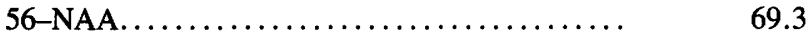

$17-$ ICP .............................

$23-$ OES $\ldots \ldots \ldots \ldots \ldots \ldots \ldots \ldots \ldots \ldots \ldots$

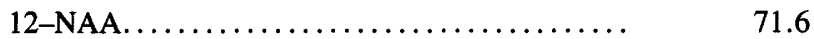

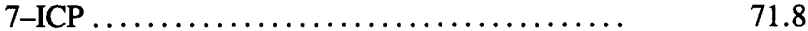

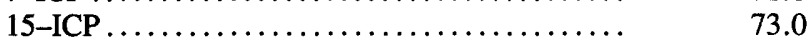

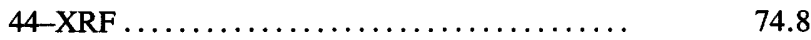

$2-X R F \ldots \ldots \ldots \ldots \ldots \ldots \ldots \ldots \ldots \ldots \ldots \ldots \ldots \ldots$

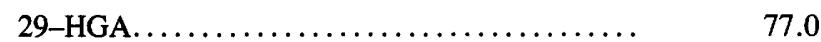

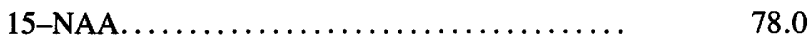

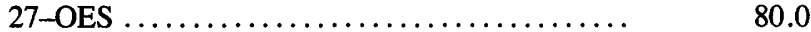

$43-$ XRF $\ldots \ldots \ldots \ldots \ldots \ldots \ldots \ldots \ldots \ldots \ldots \ldots \ldots$

$24-\mathrm{XRF} \ldots \ldots \ldots \ldots \ldots \ldots \ldots \ldots \ldots \ldots \ldots \ldots \ldots$ 


\begin{tabular}{|c|c|}
\hline \multicolumn{2}{|l|}{ Cs data } \\
\hline $\begin{array}{l}\text { Lab code/ } \\
\text { method }\end{array}$ & $\begin{array}{c}\mu \mathrm{g} / \mathrm{g} \\
\text { in sample }\end{array}$ \\
\hline 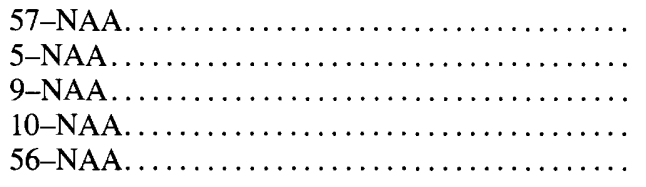 & $\begin{array}{l}5.00 \\
5.23 \\
5.97 \\
6.42 \\
6.97\end{array}$ \\
\hline 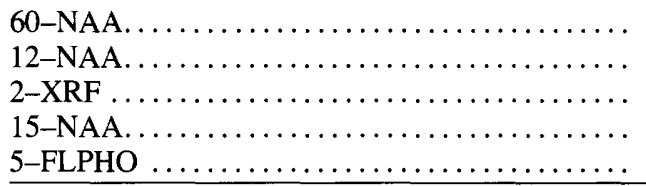 & $\begin{array}{l}7 \\
7.32 \\
7.75 \\
8.65 \\
11.5 \\
\end{array}$ \\
\hline
\end{tabular}

\begin{tabular}{|c|c|}
\hline \multicolumn{2}{|l|}{ Cu data } \\
\hline $\begin{array}{l}\text { Lab code/ } \\
\text { method }\end{array}$ & $\begin{array}{c}\mu \mathrm{g} / \mathrm{g} \\
\text { in sample }\end{array}$ \\
\hline 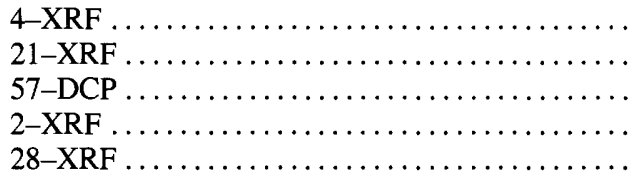 & $\begin{array}{l}29.0 \\
37.0 \\
46.0 \\
47.5 \\
50.0\end{array}$ \\
\hline 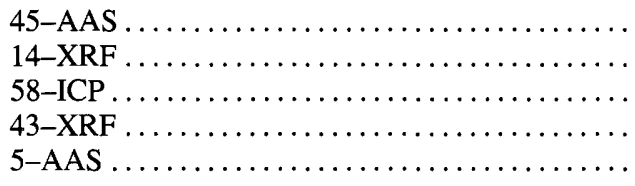 & $\begin{array}{l}50.3 \\
51.8 \\
53.5 \\
54.3 \\
54.5\end{array}$ \\
\hline 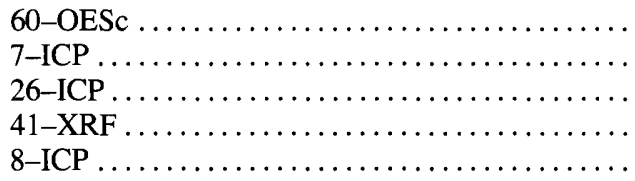 & $\begin{array}{l}55 \\
56.8 \\
58.0 \\
58.3 \\
58.3\end{array}$ \\
\hline 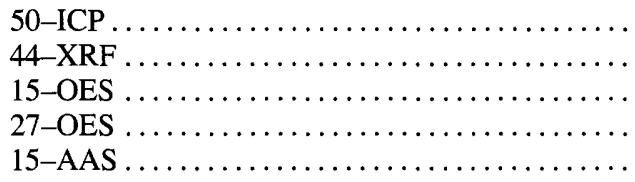 & $\begin{array}{l}58.7 \\
59.3 \\
60.0 \\
60.0 \\
61.0\end{array}$ \\
\hline 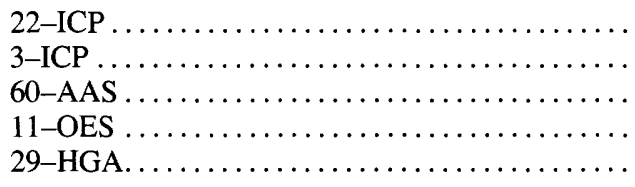 & $\begin{array}{l}61.3 \\
62.0 \\
63 \\
64.8 \\
67.0\end{array}$ \\
\hline 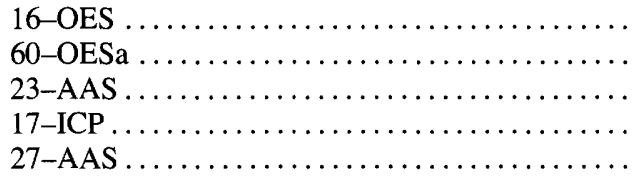 & $\begin{array}{l}69.5 \\
73 \\
74.0 \\
74.5 \\
75.0\end{array}$ \\
\hline $\begin{array}{l}40-\text { OES } 1 \ldots \\
42-\text { OES } \ldots \ldots \ldots \ldots\end{array}$ & $\begin{array}{l}75.5 \\
225\end{array}$ \\
\hline
\end{tabular}

\begin{tabular}{|c|c|}
\hline \multicolumn{2}{|l|}{ Dy data } \\
\hline $\begin{array}{l}\text { Lab code/ } \\
\text { method }\end{array}$ & $\begin{array}{c}\mu \mathrm{g} / \mathrm{g} \\
\text { in sample }\end{array}$ \\
\hline 3-ICP . & 4.75 \\
\hline $8-$ SEPN/ICP $\ldots \ldots \ldots \ldots \ldots \ldots \ldots \ldots$ & 5.20 \\
\hline $57-\mathrm{ICPMS} \ldots \ldots \ldots \ldots \ldots \ldots \ldots \ldots \ldots \ldots$ & 5.80 \\
\hline $63-I C P M S . \ldots \ldots \ldots \ldots \ldots \ldots \ldots \ldots \ldots$ & 5.90 \\
\hline $26-$ SEPN/ICP $\ldots \ldots \ldots \ldots \ldots \ldots \ldots \ldots \ldots$ & 6.00 \\
\hline 24-SEPN/ICP ......... & 6.07 \\
\hline 49-SEPN/ICP . . . . . . . . . . . . . . . . & 6.10 \\
\hline 14-SEPN/ICP $\ldots \ldots \ldots \ldots \ldots \ldots \ldots \ldots \ldots \ldots$ & 6.25 \\
\hline 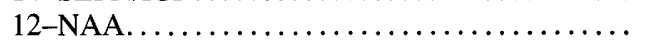 & 6.50 \\
\hline 17-SEPN/ICP $\ldots \ldots \ldots \ldots \ldots \ldots \ldots \ldots \ldots$ & 6.72 \\
\hline $7-\mathrm{NAA} \ldots \ldots \ldots \ldots \ldots$ & 6.90 \\
\hline $60-N A A \ldots \ldots \ldots \ldots$ & 7.1 \\
\hline 14-EXTR/ICP .... & 7.11 \\
\hline 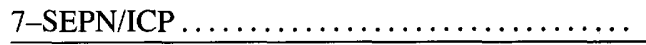 & 7.30 \\
\hline \multicolumn{2}{|l|}{ Er data } \\
\hline $\begin{array}{l}\text { Lab code/ } \\
\text { method }\end{array}$ & $\begin{array}{c}\mu \mathrm{g} / \mathrm{g} \\
\text { in sample }\end{array}$ \\
\hline 8-SEPN/ICP. & 2.90 \\
\hline 7-SEPN/ICP . & 3.10 \\
\hline 49-SEPN/ICP $\ldots \ldots \ldots \ldots \ldots \ldots \ldots \ldots \ldots$ & 3.26 \\
\hline 26-SEPN/ICP $\ldots \ldots \ldots \ldots \ldots \ldots \ldots \ldots \ldots$ & 3.40 \\
\hline $57-$ ICPMS $\ldots \ldots \ldots \ldots \ldots \ldots \ldots \ldots \ldots \ldots \ldots$ & 3.40 \\
\hline 17-SEPN/ICP . . & 3.55 \\
\hline $14-$ SEPN/ICP $\ldots \ldots \ldots \ldots \ldots \ldots \ldots \ldots \ldots$ & 3.81 \\
\hline 14-SEPN/ICP $\ldots \ldots \ldots \ldots \ldots \ldots \ldots \ldots \ldots$ & 3.98 \\
\hline $3-\mathrm{ICP} \ldots \ldots \ldots \ldots \ldots \ldots \ldots \ldots \ldots \ldots \ldots \ldots \ldots$ & 4.75 \\
\hline \multicolumn{2}{|l|}{ Eu data } \\
\hline $\begin{array}{l}\text { Lab code/ } \\
\text { method }\end{array}$ & $\begin{array}{c}\mu \mathrm{g} / \mathrm{g} \\
\text { in sample }\end{array}$ \\
\hline 3-ICP . & $<1$ \\
\hline 9-NAA $\ldots \ldots \ldots \ldots \ldots \ldots \ldots \ldots \ldots \ldots$ & 1.43 \\
\hline $5-N A A \ldots \ldots \ldots \ldots \ldots \ldots \ldots \ldots \ldots \ldots$ & 1.46 \\
\hline 10-NAA $\ldots \ldots \ldots \ldots \ldots \ldots \ldots \ldots \ldots \ldots \ldots \ldots$ & 1.47 \\
\hline 14-SEPN/ICP $\ldots \ldots \ldots \ldots \ldots \ldots \ldots \ldots \ldots$ & 1.47 \\
\hline $7-$ NAA $\ldots \ldots \ldots \ldots \ldots \ldots \ldots \ldots \ldots \ldots$ & 1.50 \\
\hline $12-N A A \ldots \ldots \ldots \ldots \ldots \ldots \ldots \ldots \ldots \ldots$ & 1.63 \\
\hline 49-SEPN/ICP $\ldots \ldots \ldots \ldots \ldots \ldots \ldots \ldots \ldots \ldots$ & 1.65 \\
\hline 14-EXTR/ICP $\ldots \ldots \ldots \ldots \ldots \ldots \ldots \ldots \ldots \ldots$ & 1.67 \\
\hline 24-SEPN/ICP $\ldots \ldots \ldots \ldots \ldots \ldots \ldots \ldots \ldots$ & 1.68 \\
\hline $50-\mathrm{ICP} \ldots \ldots \ldots \ldots \ldots \ldots \ldots \ldots \ldots \ldots \ldots$ & 1.68 \\
\hline $63-I C P M S . \ldots \ldots \ldots \ldots \ldots \ldots \ldots \ldots \ldots \ldots \ldots$ & 1.68 \\
\hline 26-SEPN/ICP $\ldots \ldots \ldots \ldots \ldots \ldots \ldots \ldots \ldots \ldots$ & 1.70 \\
\hline $57-$ ICPMS $\ldots \ldots \ldots \ldots \ldots \ldots \ldots \ldots \ldots \ldots$ & 1.71 \\
\hline $8-$ SEPN/ICP $\ldots \ldots \ldots \ldots \ldots \ldots \ldots \ldots \ldots$ & 1.72 \\
\hline 56-NAA. . & 1.77 \\
\hline $60-N A A, \ldots \ldots \ldots \ldots \ldots \ldots \ldots \ldots \ldots \ldots \ldots \ldots \ldots$ & 1.9 \\
\hline 17-SEPN/ICP $\ldots \ldots \ldots \ldots \ldots \ldots \ldots \ldots \ldots$ & 1.95 \\
\hline $7-\mathrm{ICP} \ldots \ldots \ldots \ldots \ldots \ldots \ldots \ldots \ldots \ldots \ldots$ & $<2$ \\
\hline $7-$ SEPN/ICP $\ldots \ldots \ldots \ldots \ldots \ldots \ldots \ldots \ldots$ & 2.0 \\
\hline $15-N A A \ldots \ldots \ldots \ldots \ldots \ldots \ldots \ldots \ldots \ldots \ldots \ldots$ & 2.05 \\
\hline
\end{tabular}




\begin{tabular}{|c|c|}
\hline \multicolumn{2}{|l|}{ F data } \\
\hline $\begin{array}{l}\text { Lab code/ } \\
\text { method }\end{array}$ & $\begin{array}{c}\mu \mathrm{g} / \mathrm{g} \\
\text { in sample }\end{array}$ \\
\hline 14 & $<100$ \\
\hline 60-ISE .... & 534 \\
\hline $55-I S E \ldots \ldots \ldots \ldots \ldots \ldots$ & 590 \\
\hline 24-PIGE ............. & 687 \\
\hline $18-\ldots \ldots \ldots \ldots \ldots \ldots \ldots \ldots \ldots \ldots \ldots \ldots \ldots \ldots \ldots \ldots \ldots \ldots$ & 720 \\
\hline 4-ISE . & 727 \\
\hline $61-\mathrm{IC} \ldots$ & 742 \\
\hline $61-I C \ldots \ldots \ldots \ldots \ldots \ldots \ldots \ldots \ldots \ldots$ & 780 \\
\hline 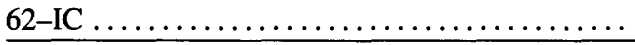 & 808 \\
\hline
\end{tabular}

\begin{tabular}{|c|c|}
\hline \multicolumn{2}{|l|}{ Hf data } \\
\hline $\begin{array}{l}\text { Lab code/ } \\
\text { method }\end{array}$ & $\begin{array}{c}\mu \mathrm{g} / \mathrm{g} \\
\text { in sample }\end{array}$ \\
\hline 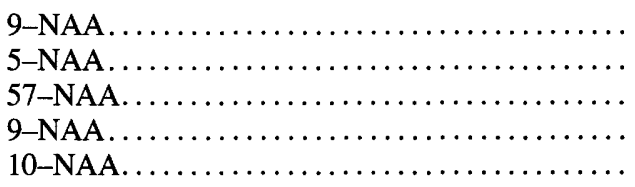 & $\begin{array}{l}3.28 \\
3.90 \\
4.00 \\
4.03 \\
4.38\end{array}$ \\
\hline 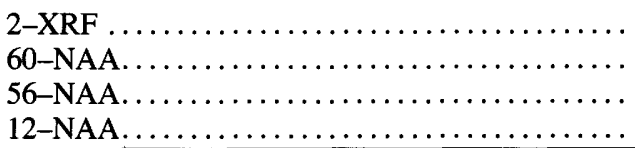 & $\begin{array}{l}4.50 \\
4.6 \\
4.62 \\
5.80\end{array}$ \\
\hline
\end{tabular}

\begin{tabular}{|c|c|}
\hline \multicolumn{2}{|l|}{ Ga data } \\
\hline $\begin{array}{l}\text { Lab code/ } \\
\text { method }\end{array}$ & $\begin{array}{c}\mu \mathrm{g} / \mathrm{g} \\
\text { in sample }\end{array}$ \\
\hline 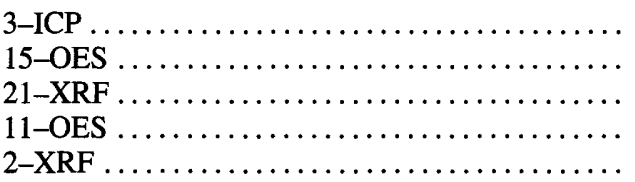 & $\begin{array}{r}<10 \\
14.0 \\
15.0 \\
16.0 \\
16.5\end{array}$ \\
\hline 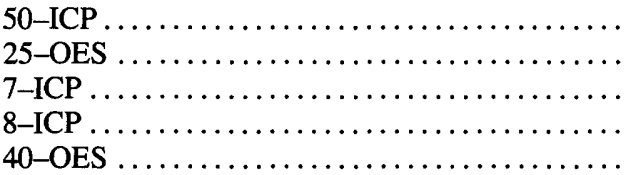 & $\begin{array}{l}16.7 \\
16.8 \\
17.3 \\
17.3 \\
18.5\end{array}$ \\
\hline 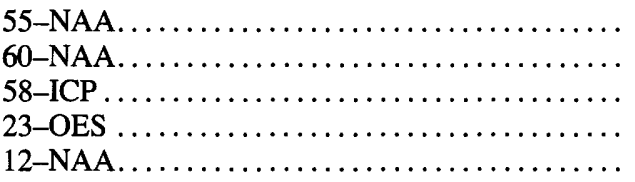 & $\begin{array}{l}19.0 \\
19 \\
<20.0 \\
20.0 \\
23.6\end{array}$ \\
\hline 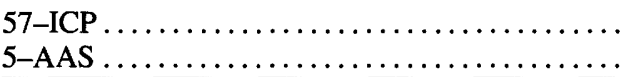 & $\begin{array}{l}24.0 \\
27.5\end{array}$ \\
\hline
\end{tabular}

\begin{tabular}{|c|c|}
\hline \multicolumn{2}{|l|}{ Gd data } \\
\hline $\begin{array}{l}\text { Lab code/ } \\
\text { method }\end{array}$ & $\begin{array}{c}\mu \mathrm{g} / \mathrm{g} \\
\text { in sample }\end{array}$ \\
\hline 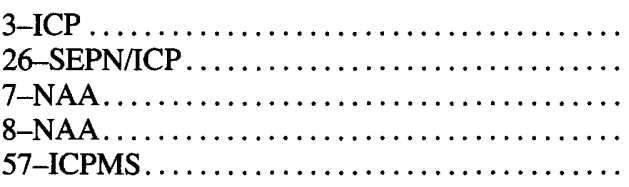 & $\begin{array}{l}5.0 \\
5.1 \\
6.2 \\
6.9 \\
6.9\end{array}$ \\
\hline 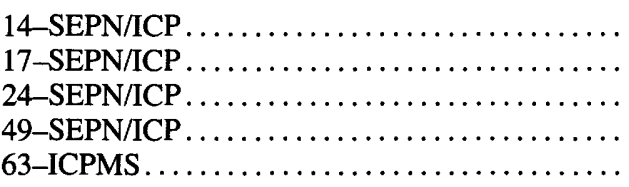 & $\begin{array}{l}7.1 \\
7.3 \\
7.8 \\
7.8 \\
7.8\end{array}$ \\
\hline 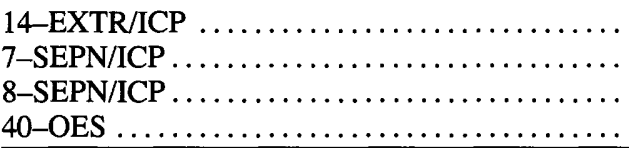 & $\begin{array}{r}8.1 \\
9.1 \\
9.7 \\
11.5\end{array}$ \\
\hline
\end{tabular}

\begin{tabular}{|c|c|}
\hline \multicolumn{2}{|l|}{ Hg data } \\
\hline $\begin{array}{l}\text { Lab code/ } \\
\text { method }\end{array}$ & $\begin{array}{c}\mu \mathrm{g} / \mathrm{g} \\
\text { in sample }\end{array}$ \\
\hline 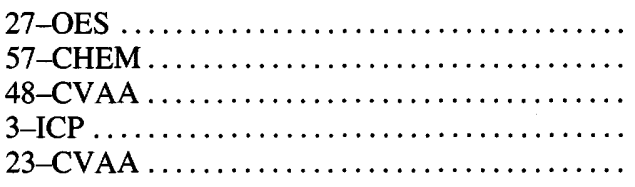 & $\begin{array}{c}<0.05 \\
.088 \\
.096 \\
.18 \\
.19\end{array}$ \\
\hline 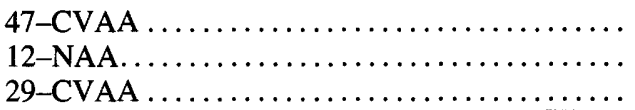 & $\begin{array}{l}.29 \\
1.3 \\
3.0\end{array}$ \\
\hline
\end{tabular}

\begin{tabular}{|c|c|}
\hline \multicolumn{2}{|l|}{ Ho data } \\
\hline $\begin{array}{l}\text { Lab code/ } \\
\text { method }\end{array}$ & $\begin{array}{c}\mu \mathrm{g} / \mathrm{g} \\
\text { in sample }\end{array}$ \\
\hline 3-ICP . & $<1$ \\
\hline 8-SEPN/ICP . & 1.07 \\
\hline $57-I C P M S . . . \ldots \ldots \ldots \ldots$ & 1.08 \\
\hline 49-SEPN/ICP ...................... & 1.14 \\
\hline $14-$ SEPN/ICP $\ldots \ldots \ldots \ldots \ldots \ldots \ldots \ldots \ldots$ & 1.19 \\
\hline 7-SEPN/ICP ..... & 1.2 \\
\hline 26-SEPN/ICP ......... & 1.3 \\
\hline 14-EXTR/ICP ......... & 1.38 \\
\hline $7-\mathrm{ICP} \ldots \ldots \ldots \ldots \ldots \ldots$ & $<4$ \\
\hline
\end{tabular}




\begin{tabular}{|c|c|}
\hline \multicolumn{2}{|l|}{ La data } \\
\hline $\begin{array}{l}\text { Lab code/ } \\
\text { method }\end{array}$ & $\begin{array}{c}\mu \mathrm{g} / \mathrm{g} \\
\text { in sample }\end{array}$ \\
\hline 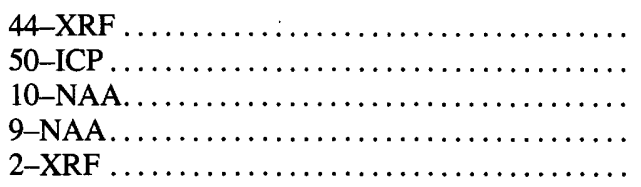 & $\begin{array}{l}26.7 \\
33.1 \\
33.3 \\
33.7 \\
33.8\end{array}$ \\
\hline 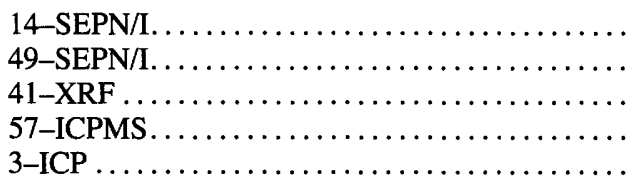 & $\begin{array}{l}35.0 \\
35.2 \\
35.5 \\
35.5 \\
35.5\end{array}$ \\
\hline 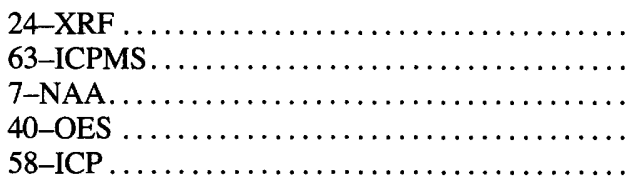 & $\begin{array}{l}35.9 \\
35.9 \\
36.0 \\
36.2 \\
37.0\end{array}$ \\
\hline 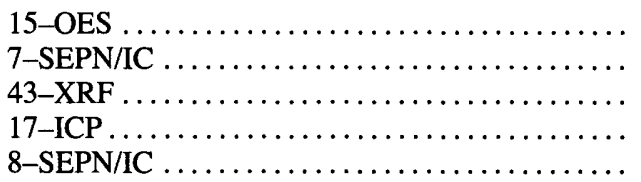 & $\begin{array}{l}37.0 \\
37.4 \\
37.5 \\
37.8 \\
37.9\end{array}$ \\
\hline 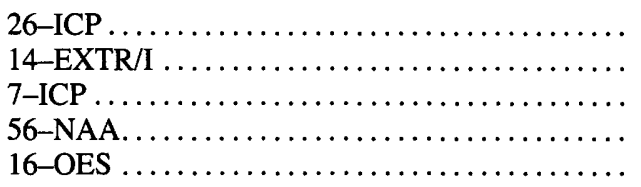 & $\begin{array}{l}38.0 \\
38.5 \\
38.8 \\
39.1 \\
39.5\end{array}$ \\
\hline 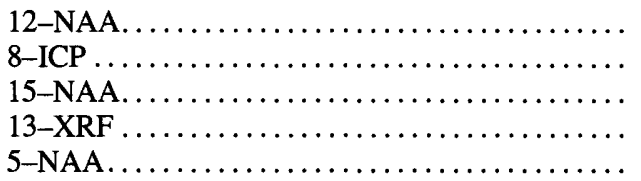 & $\begin{array}{l}40.2 \\
41.0 \\
41.5 \\
41.7 \\
42.8\end{array}$ \\
\hline 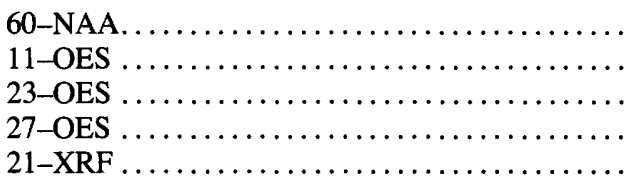 & $\begin{array}{c}42.8 \\
45.3 \\
50.0 \\
<60 \\
70.0\end{array}$ \\
\hline
\end{tabular}

\begin{tabular}{|c|c|}
\hline \multicolumn{2}{|l|}{ Li data } \\
\hline $\begin{array}{l}\text { Lab code/ } \\
\text { method }\end{array}$ & $\begin{array}{c}\mu \mathrm{g} / \mathrm{g} \\
\text { in sample }\end{array}$ \\
\hline 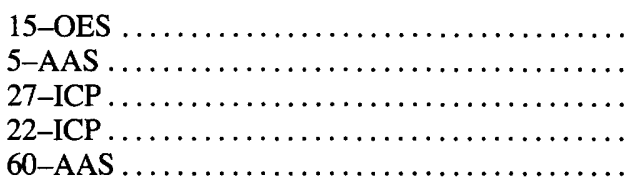 & $\begin{array}{l}21.62 \\
22.5 \\
28.0 \\
31.2 \\
31.8\end{array}$ \\
\hline 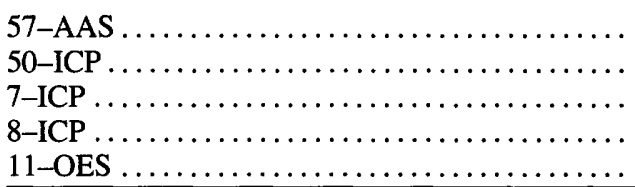 & $\begin{array}{l}32.0 \\
32.7 \\
33.7 \\
34.2 \\
43.2\end{array}$ \\
\hline
\end{tabular}

\begin{tabular}{|c|c|}
\hline \multicolumn{2}{|l|}{ Lu data } \\
\hline $\begin{array}{l}\text { Lab code/ } \\
\text { method }\end{array}$ & $\begin{array}{c}\mu \mathrm{g} / \mathrm{g} \\
\text { in sample }\end{array}$ \\
\hline 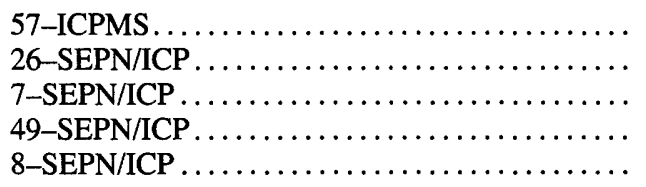 & $\begin{array}{r}0.42 \\
.43 \\
.43 \\
.44 \\
.45\end{array}$ \\
\hline 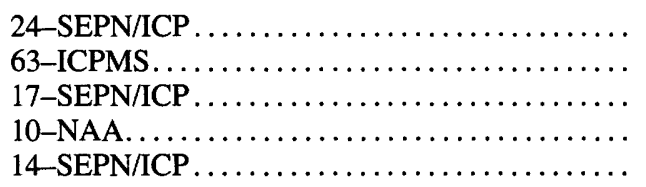 & $\begin{array}{l}.45 \\
.45 \\
.47 \\
.48 \\
.49\end{array}$ \\
\hline 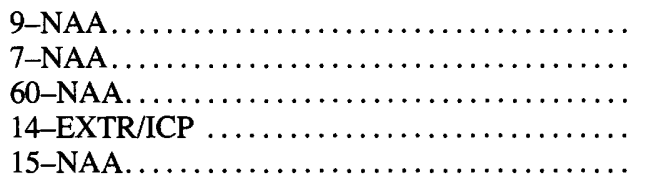 & $\begin{array}{l}.50 \\
.53 \\
.54 \\
.55 \\
.63\end{array}$ \\
\hline 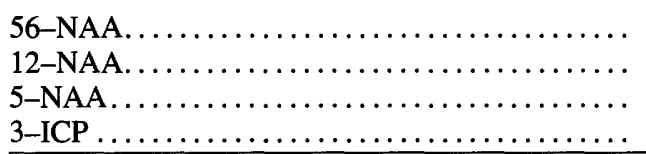 & $\begin{array}{l}.68 \\
.69 \\
.96 \\
4.0 \\
\end{array}$ \\
\hline
\end{tabular}

\begin{tabular}{|c|c|}
\hline \multicolumn{2}{|l|}{ Mo data } \\
\hline $\begin{array}{l}\text { Lab code/ } \\
\text { method }\end{array}$ & $\begin{array}{c}\mu \mathrm{g} / \mathrm{g} \\
\text { in sample }\end{array}$ \\
\hline 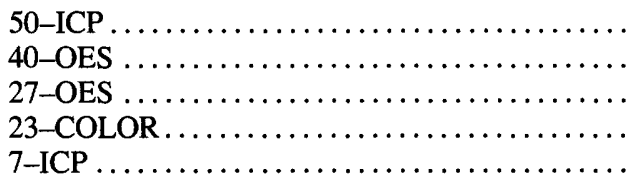 & $\begin{array}{l}108 \\
109 \\
120 \\
122 \\
122\end{array}$ \\
\hline 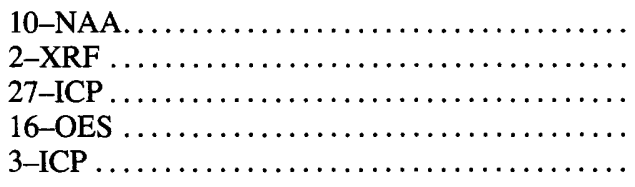 & $\begin{array}{l}126 \\
131 \\
132 \\
133 \\
134\end{array}$ \\
\hline 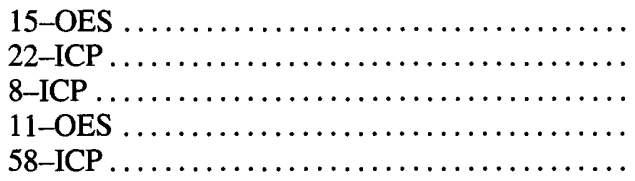 & $\begin{array}{l}140 \\
142 \\
148 \\
150 \\
150\end{array}$ \\
\hline 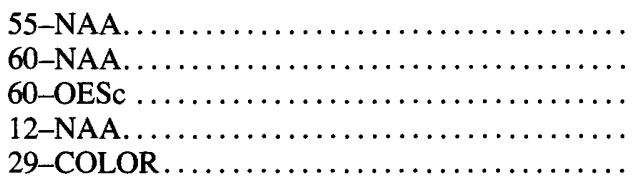 & $\begin{array}{l}155 \\
157 \\
160 \\
161 \\
181\end{array}$ \\
\hline 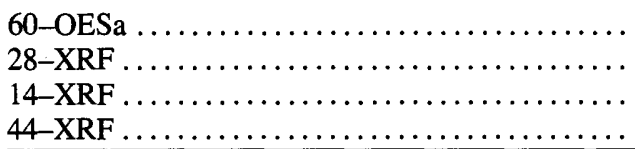 & $\begin{array}{l}190 \\
193 \\
198 \\
215\end{array}$ \\
\hline
\end{tabular}




\begin{tabular}{|c|c|}
\hline \multicolumn{2}{|l|}{ Nb data } \\
\hline $\begin{array}{l}\text { Lab code/ } \\
\text { method }\end{array}$ & $\begin{array}{c}\mu \mathrm{g} / \mathrm{g} \\
\text { in sample }\end{array}$ \\
\hline 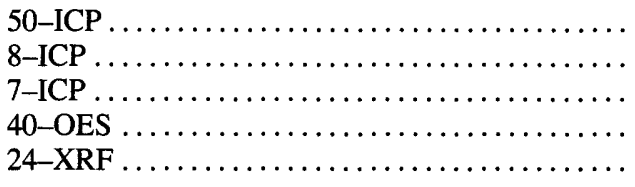 & $\begin{array}{r}5.3 \\
6.0 \\
6.3 \\
9.5 \\
10.0\end{array}$ \\
\hline 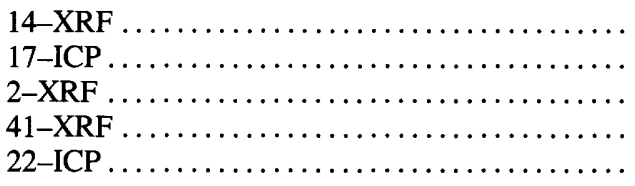 & $\begin{array}{l}10.8 \\
12.0 \\
12.0 \\
12.3 \\
12.3\end{array}$ \\
\hline 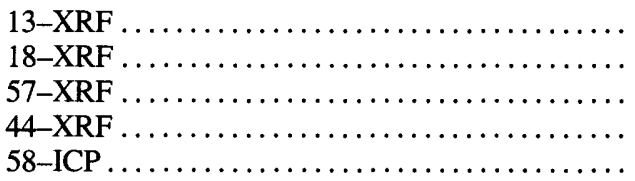 & $\begin{array}{l}12.3 \\
12.5 \\
17.0 \\
17.1 \\
20.0\end{array}$ \\
\hline 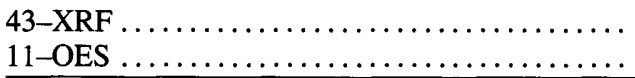 & $\begin{aligned} & 20.3 \\
&<25\end{aligned}$ \\
\hline
\end{tabular}

\begin{tabular}{|c|c|}
\hline \multicolumn{2}{|l|}{ Nd data } \\
\hline $\begin{array}{l}\text { Lab code/ } \\
\text { method }\end{array}$ & $\begin{array}{c}\mu \mathrm{g} / \mathrm{g} \\
\text { in sample }\end{array}$ \\
\hline 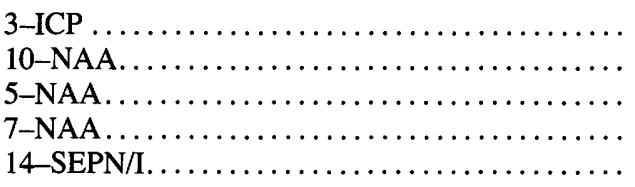 & $\begin{array}{l}30.8 \\
32.2 \\
33.0 \\
35.0 \\
35.6\end{array}$ \\
\hline 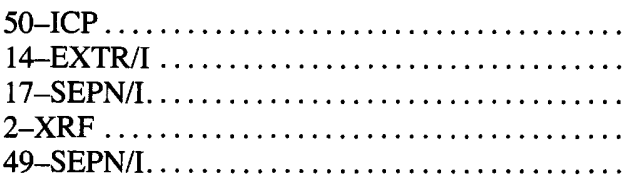 & $\begin{array}{l}35.7 \\
35.7 \\
36.2 \\
36.2 \\
36.3\end{array}$ \\
\hline 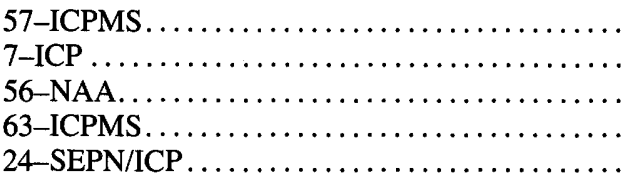 & $\begin{array}{l}37.0 \\
37.2 \\
37.4 \\
38.2 \\
38.3\end{array}$ \\
\hline 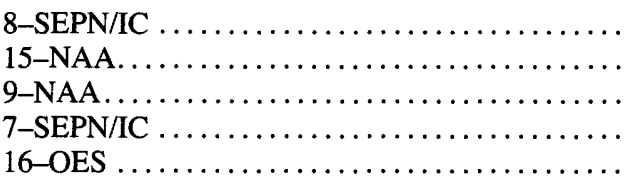 & $\begin{array}{l}38.6 \\
38.8 \\
39.3 \\
39.4 \\
40.0\end{array}$ \\
\hline $12-N A A . \ldots \ldots \ldots \ldots \ldots \ldots \ldots \ldots \ldots \ldots$ & 40.4 \\
\hline
\end{tabular}

\begin{tabular}{|c|c|}
\hline \multicolumn{2}{|l|}{ Ni data } \\
\hline $\begin{array}{l}\text { Lab code/ } \\
\text { method }\end{array}$ & $\begin{array}{c}\mu \mathrm{g} / \mathrm{g} \\
\text { in sample }\end{array}$ \\
\hline 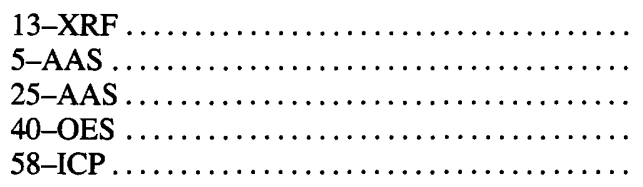 & $\begin{array}{l}84.0 \\
86.3 \\
88.5 \\
89.3 \\
89.5\end{array}$ \\
\hline 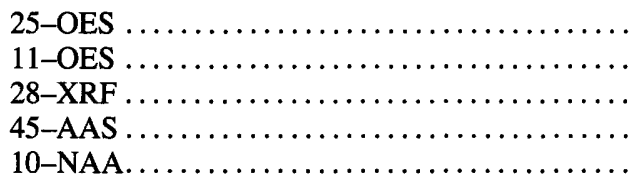 & $\begin{array}{l}89.9 \\
90.0 \\
90.0 \\
90.3 \\
91.8\end{array}$ \\
\hline 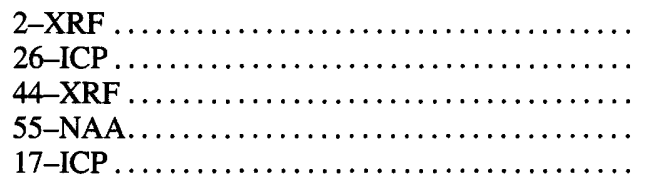 & $\begin{array}{l}93.3 \\
94.0 \\
94.9 \\
98 \\
99.3\end{array}$ \\
\hline 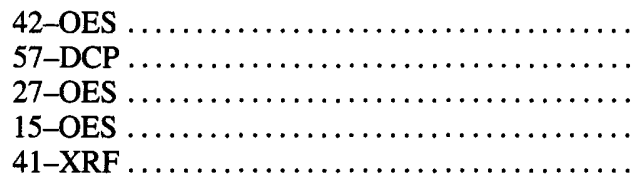 & $\begin{array}{l}99.8 \\
100 \\
100 \\
101 \\
102\end{array}$ \\
\hline 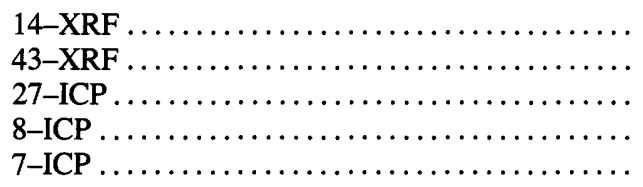 & $\begin{array}{l}106 \\
108 \\
109 \\
110 \\
110\end{array}$ \\
\hline 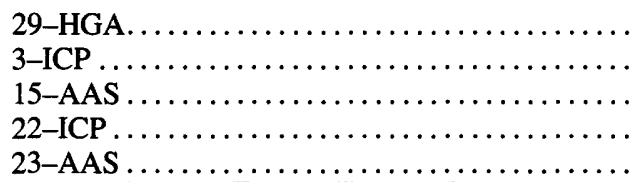 & $\begin{array}{l}111 \\
116 \\
117 \\
128 \\
128\end{array}$ \\
\hline
\end{tabular}




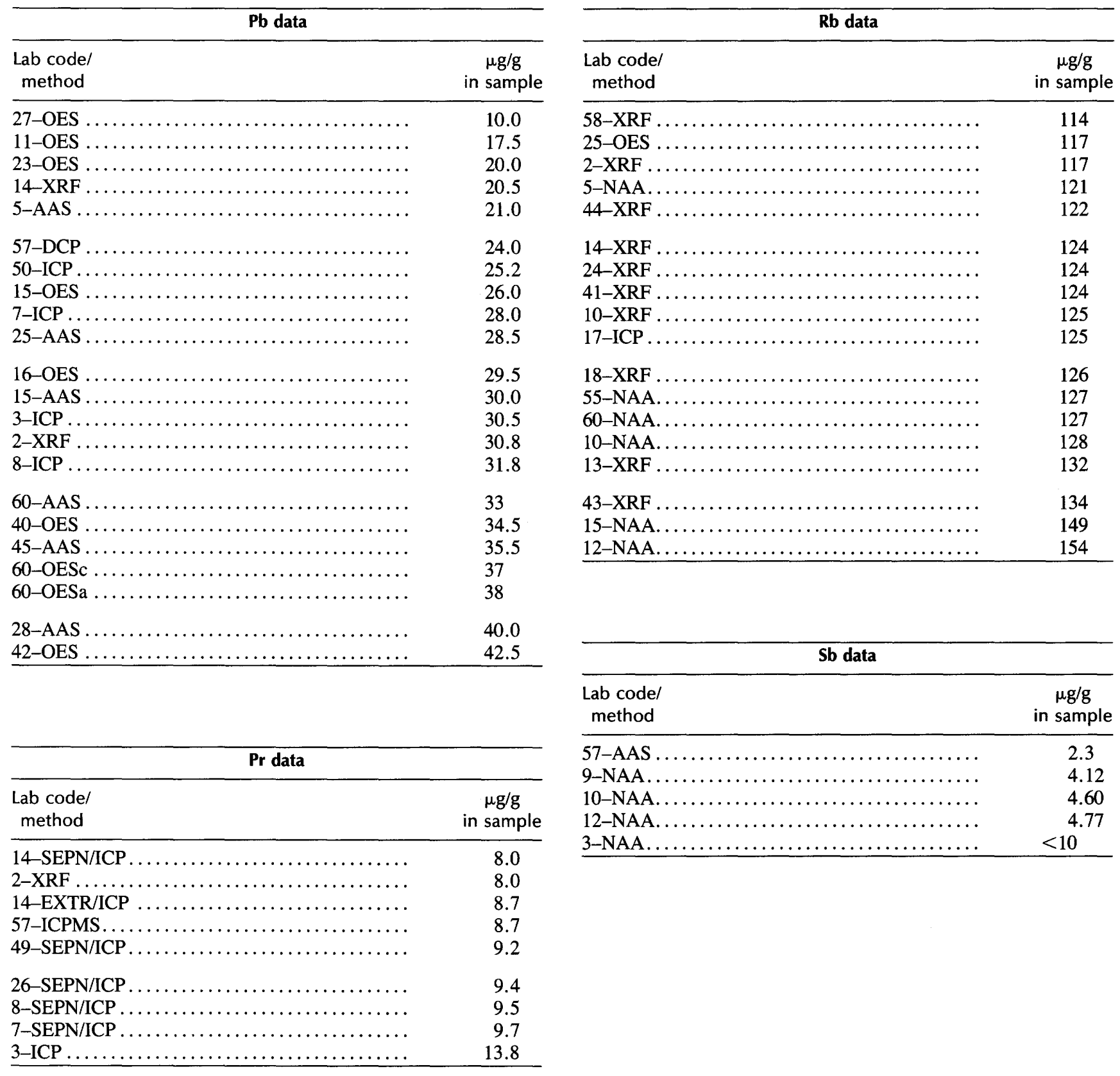




Sc data

\begin{tabular}{|c|c|}
\hline $\begin{array}{l}\text { Lab code/ } \\
\text { method }\end{array}$ & $\begin{array}{c}\mu \mathrm{g} / \mathrm{g} \\
\text { in sample }\end{array}$ \\
\hline 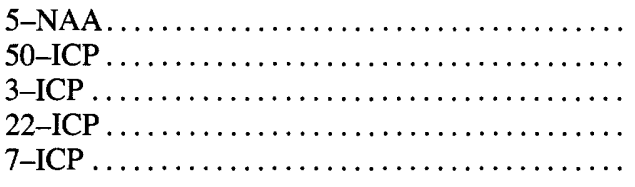 & $\begin{array}{l}10.3 \\
11.8 \\
11.8 \\
12.0 \\
12.0\end{array}$ \\
\hline 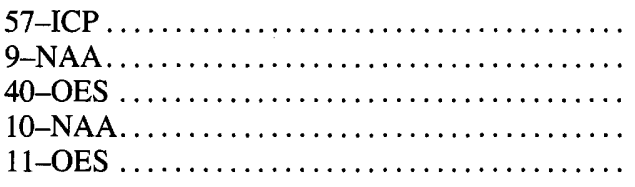 & $\begin{array}{l}12.0 \\
12.3 \\
12.6 \\
12.7 \\
12.8\end{array}$ \\
\hline 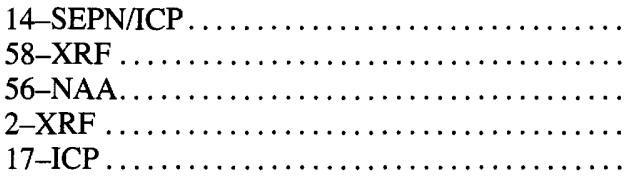 & $\begin{array}{l}12.8 \\
14.0 \\
14.0 \\
14.3 \\
14.3\end{array}$ \\
\hline 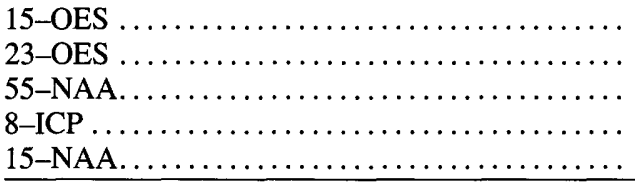 & $\begin{array}{l}14.4 \\
15.0 \\
15.2 \\
15.3 \\
18.3\end{array}$ \\
\hline
\end{tabular}

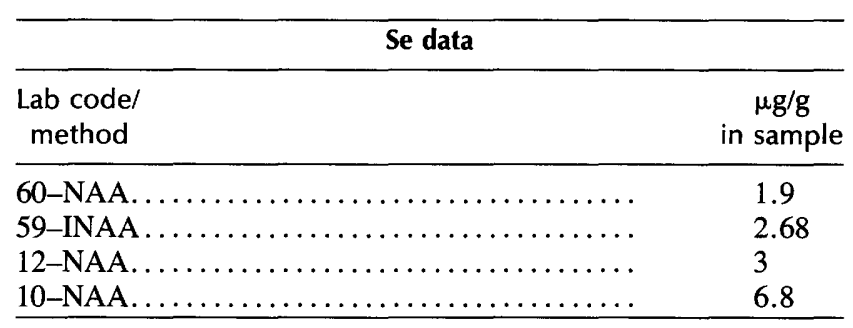

\begin{tabular}{|c|c|}
\hline \multicolumn{2}{|l|}{ Sm data } \\
\hline $\begin{array}{l}\text { Lab code/ } \\
\text { method }\end{array}$ & $\begin{array}{c}\mu \mathrm{g} / \mathrm{g} \\
\text { in sample }\end{array}$ \\
\hline 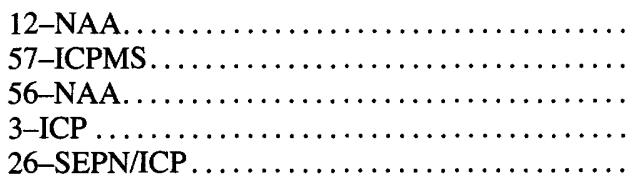 & $\begin{array}{l}6.4 \\
6.8 \\
7.41 \\
7 \\
7.5\end{array}$ \\
\hline 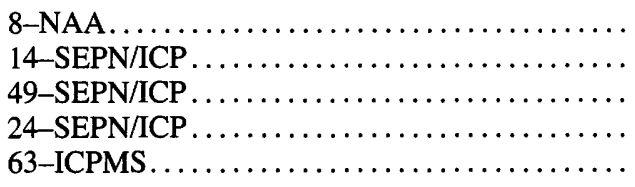 & $\begin{array}{l}7.5 \\
7.5 \\
7.6 \\
7.6 \\
7.6\end{array}$ \\
\hline 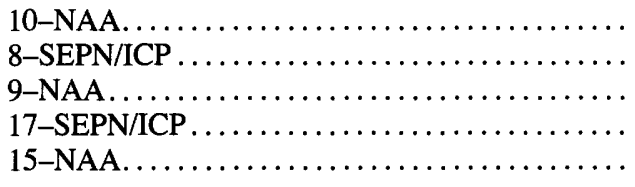 & $\begin{array}{l}7.7 \\
8.2 \\
8.3 \\
8.4 \\
8.6\end{array}$ \\
\hline 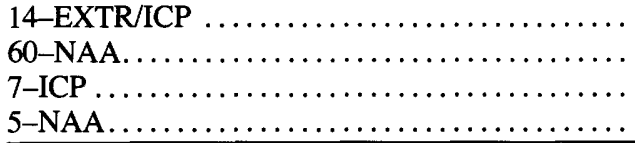 & $\begin{array}{r}8.7 \\
8.7 \\
9.4 \\
13\end{array}$ \\
\hline
\end{tabular}

\begin{tabular}{|c|c|}
\hline \multicolumn{2}{|l|}{ Sn data } \\
\hline $\begin{array}{l}\text { Lab code/ } \\
\text { method }\end{array}$ & $\begin{array}{c}\mu \mathrm{g} / \mathrm{g} \\
\text { in sample }\end{array}$ \\
\hline 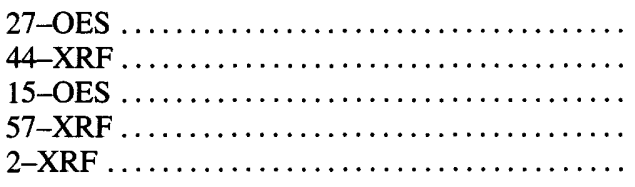 & $\begin{array}{l}2.5 \\
2.7 \\
3.2 \\
5.0 \\
3.3\end{array}$ \\
\hline 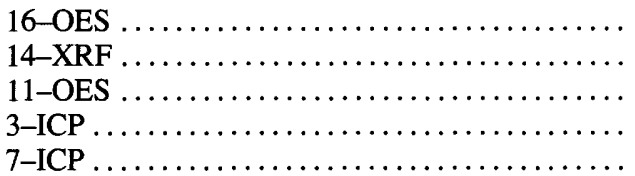 & $\begin{aligned} & 5.3 \\
< & 7 \\
< & 10 \\
< & 10 \\
< & 10\end{aligned}$ \\
\hline
\end{tabular}




\begin{tabular}{|c|c|}
\hline \multicolumn{2}{|l|}{ Sr data } \\
\hline $\begin{array}{l}\text { Lab code/ } \\
\text { method }\end{array}$ & $\begin{array}{c}\mu \mathrm{g} / \mathrm{g} \\
\text { in sample }\end{array}$ \\
\hline 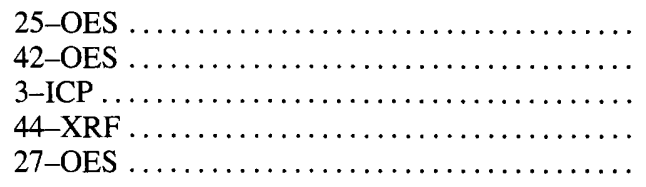 & $\begin{array}{l}31.5 \\
41.5 \\
68.0 \\
68.3 \\
70.0\end{array}$ \\
\hline 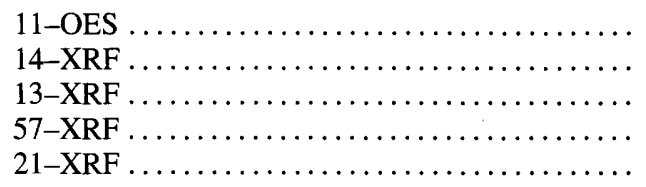 & $\begin{array}{l}72.0 \\
72.0 \\
72.7 \\
73.0 \\
73.0\end{array}$ \\
\hline 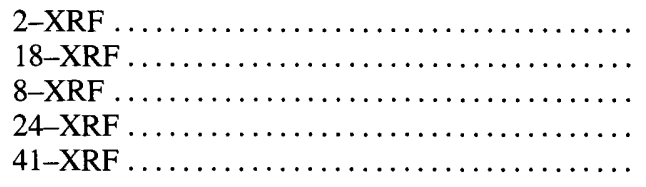 & $\begin{array}{l}73.8 \\
74.5 \\
75.8 \\
76.0 \\
76.0\end{array}$ \\
\hline 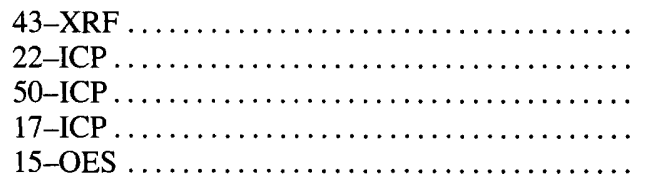 & $\begin{array}{l}76.0 \\
77.5 \\
78.3 \\
78.8 \\
80.0\end{array}$ \\
\hline 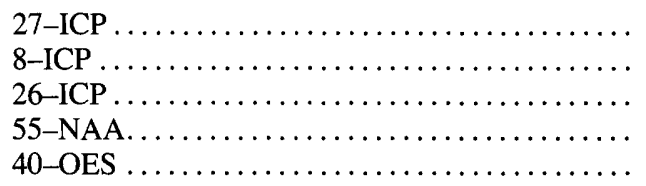 & $\begin{array}{r}80.0 \\
80.3 \\
83.5 \\
93.0 \\
106.0\end{array}$ \\
\hline 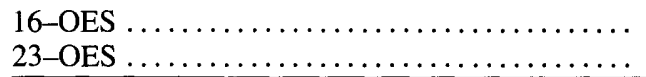 & $\begin{array}{l}128 \\
150\end{array}$ \\
\hline
\end{tabular}

\begin{tabular}{|c|c|}
\hline \multicolumn{2}{|l|}{ Ta data } \\
\hline $\begin{array}{l}\text { Lab code/ } \\
\text { method }\end{array}$ & $\begin{array}{c}\mu \mathrm{g} / \mathrm{g} \\
\text { in sample }\end{array}$ \\
\hline 60-NAA......... & 1.1 \\
\hline 9-NAA $\ldots \ldots \ldots \ldots \ldots$ & 1.03 \\
\hline $10-N A A . \ldots \ldots \ldots \ldots \ldots \ldots \ldots \ldots \ldots \ldots \ldots \ldots \ldots \ldots$ & 1.07 \\
\hline $5-N A A \ldots \ldots \ldots \ldots \ldots \ldots \ldots \ldots \ldots \ldots$ & 1.08 \\
\hline $56-N A A . \ldots \ldots \ldots \ldots \ldots \ldots \ldots \ldots \ldots \ldots \ldots \ldots \ldots \ldots$ & 1.08 \\
\hline 12-NAA. . & 1.19 \\
\hline 15-NAA..... & 1.36 \\
\hline $2-\mathrm{XRF} \ldots \ldots \ldots \ldots \ldots$ & $<3$ \\
\hline 2-XRF . & 4 \\
\hline $3-\mathrm{ICP} \ldots$. & $<10$ \\
\hline $7-\mathrm{ICP} \ldots \ldots \ldots \ldots \ldots \ldots \ldots \ldots \ldots \ldots \ldots$ & $<40$ \\
\hline
\end{tabular}

\begin{tabular}{|c|c|}
\hline \multicolumn{2}{|l|}{ Tb data } \\
\hline $\begin{array}{l}\text { Lab code/ } \\
\text { method }\end{array}$ & $\begin{array}{c}\mu \mathrm{g} / \mathrm{g} \\
\text { in sample }\end{array}$ \\
\hline 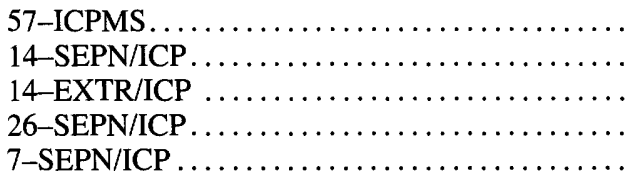 & $\begin{array}{r}0.80 \\
.93 \\
.96 \\
1.00 \\
1.00\end{array}$ \\
\hline 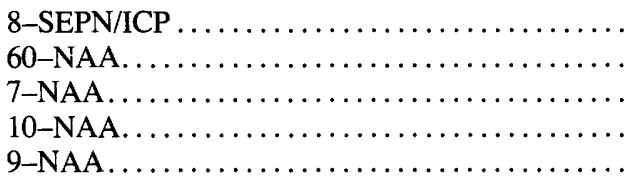 & $\begin{array}{l}1.00 \\
1.2 \\
1.10 \\
1.15 \\
1.16\end{array}$ \\
\hline 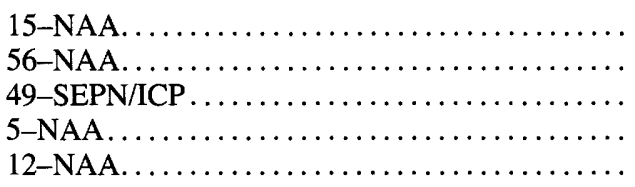 & $\begin{array}{l}1.26 \\
1.28 \\
1.40 \\
1.46 \\
1.65\end{array}$ \\
\hline 3-ICP .... & 3.25 \\
\hline
\end{tabular}

\begin{tabular}{|c|c|}
\hline \multicolumn{2}{|l|}{ Th data } \\
\hline $\begin{array}{l}\text { Lab code/ } \\
\text { method }\end{array}$ & $\begin{array}{c}\mu \mathrm{g} / \mathrm{g} \\
\text { in sample }\end{array}$ \\
\hline 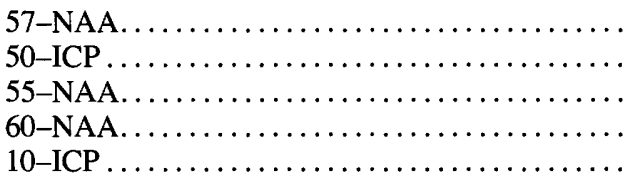 & $\begin{array}{l}8.10 \\
9.20 \\
9.5 \\
9.5 \\
9.77\end{array}$ \\
\hline 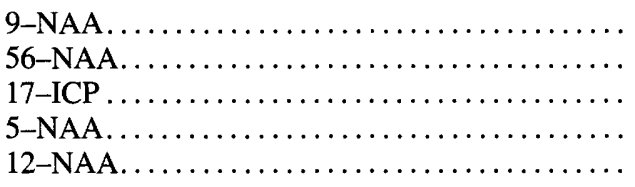 & $\begin{array}{r}9.82 \\
9.95 \\
10.35 \\
10.60 \\
10.62\end{array}$ \\
\hline 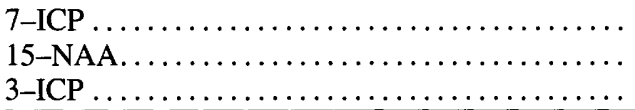 & $\begin{array}{l}11.00 \\
11.21 \\
26.25\end{array}$ \\
\hline
\end{tabular}

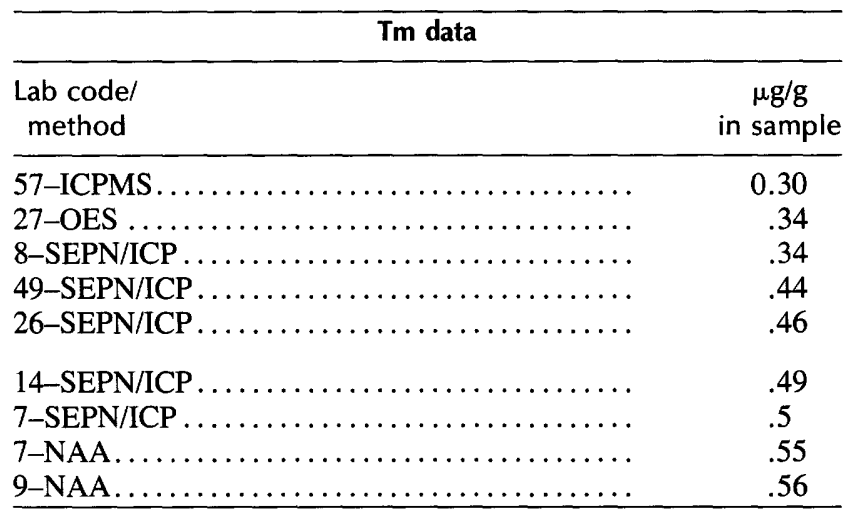




\begin{tabular}{|c|c|}
\hline \multicolumn{2}{|l|}{ U data } \\
\hline $\begin{array}{l}\text { Lab code/ } \\
\text { method }\end{array}$ & $\begin{array}{c}\mu \mathrm{g} / \mathrm{g} \\
\text { in sample }\end{array}$ \\
\hline 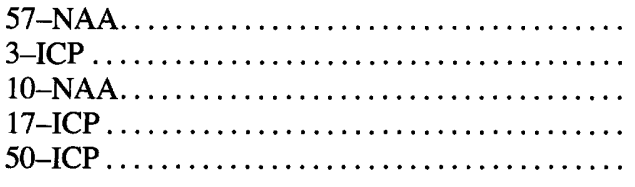 & $\begin{array}{l}34.2 \\
40.5 \\
40.8 \\
43.2 \\
44.8\end{array}$ \\
\hline 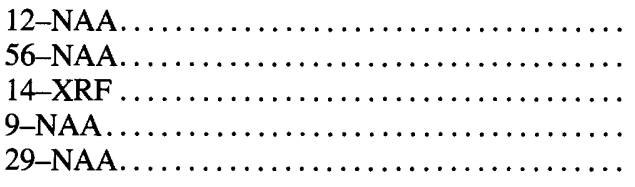 & $\begin{array}{l}46.2 \\
46.6 \\
48.5 \\
50.7 \\
51.0\end{array}$ \\
\hline 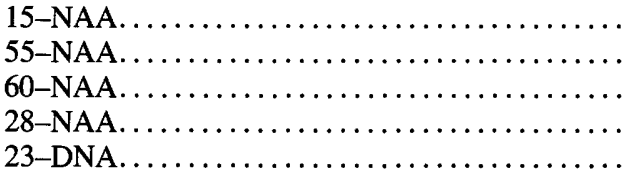 & $\begin{array}{l}51.8 \\
52 \\
52 \\
54.0 \\
55.6\end{array}$ \\
\hline 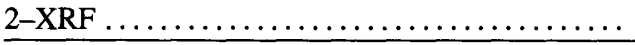 & 61.5 \\
\hline
\end{tabular}

\begin{tabular}{|c|c|}
\hline \multicolumn{2}{|l|}{$\checkmark$ data } \\
\hline $\begin{array}{l}\text { Lab code/ } \\
\text { method }\end{array}$ & $\begin{array}{c}\mu \mathrm{g} / \mathrm{g} \\
\text { in sample }\end{array}$ \\
\hline 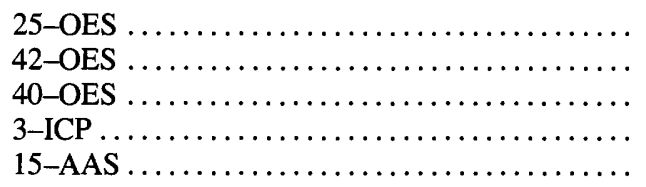 & $\begin{array}{r}88 \\
123 \\
133 \\
139 \\
145\end{array}$ \\
\hline 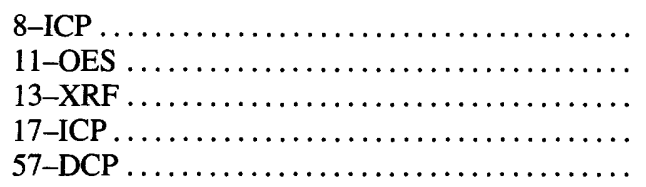 & $\begin{array}{l}158 \\
158 \\
158 \\
159 \\
160\end{array}$ \\
\hline 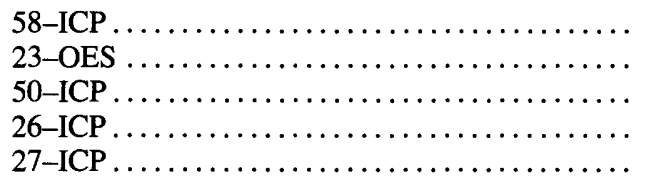 & $\begin{array}{l}160 \\
161 \\
164 \\
165 \\
166\end{array}$ \\
\hline 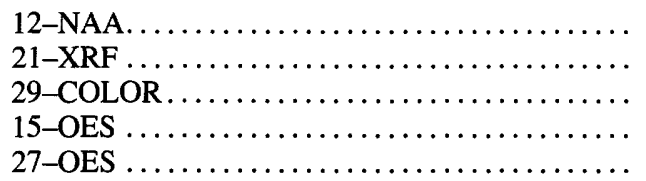 & $\begin{array}{l}167 \\
168 \\
168 \\
170 \\
170\end{array}$ \\
\hline 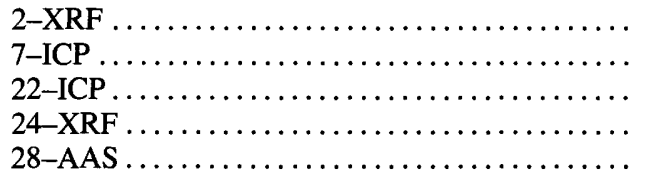 & $\begin{array}{l}172 \\
179 \\
179 \\
219 \\
266\end{array}$ \\
\hline
\end{tabular}

\begin{tabular}{|c|c|}
\hline \multicolumn{2}{|l|}{ W data } \\
\hline $\begin{array}{l}\text { Lab code/ } \\
\text { method }\end{array}$ & $\begin{array}{c}\mu \mathrm{g} / \mathrm{g} \\
\text { in sample }\end{array}$ \\
\hline 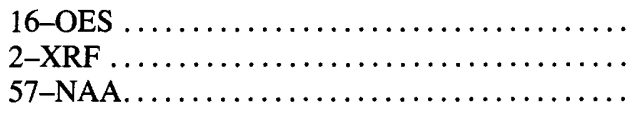 & $\begin{array}{l}<0.8 \\
<3 \\
<3\end{array}$ \\
\hline 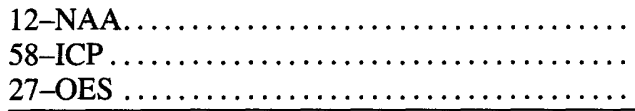 & $\begin{array}{r}3.34 \\
5 \\
<60 \\
\end{array}$ \\
\hline
\end{tabular}

\begin{tabular}{lc}
\hline \multicolumn{2}{c}{ Y data } \\
\hline $\begin{array}{c}\mu \mathrm{g} / \mathrm{g} \\
\text { method }\end{array}$ & in sample
\end{tabular}

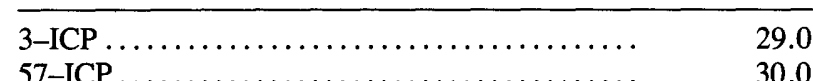

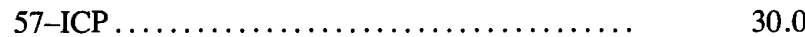

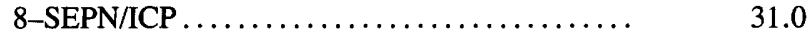

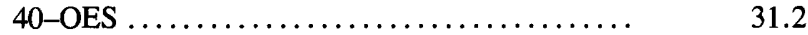

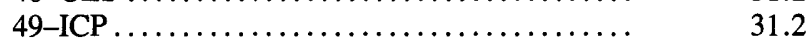

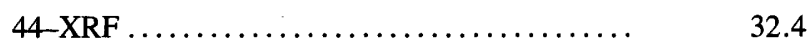

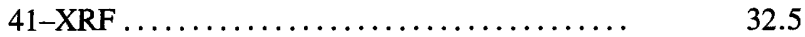

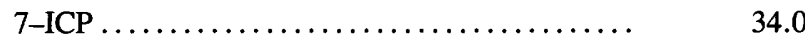

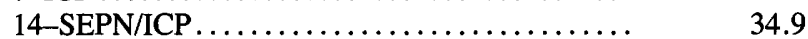

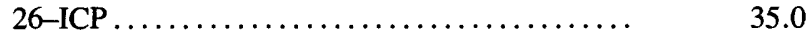

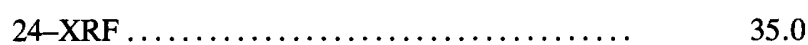

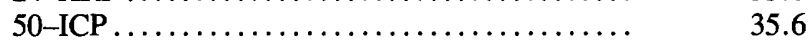

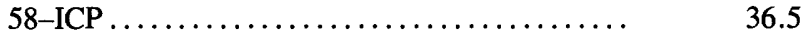

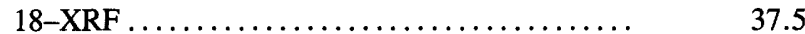

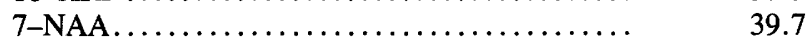

$13-\mathrm{XRF} \ldots \ldots \ldots \ldots \ldots \ldots \ldots \ldots \ldots \ldots \ldots \ldots . \ldots \ldots$

14-EXTR/ICP $\ldots \ldots \ldots \ldots \ldots \ldots \ldots \ldots \ldots \ldots . \ldots . \ldots . \ldots$

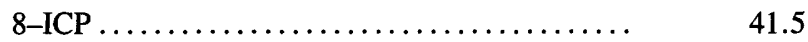

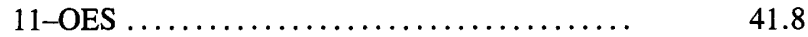

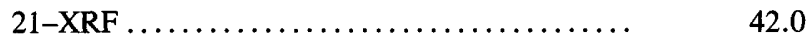

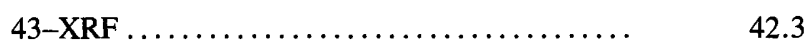

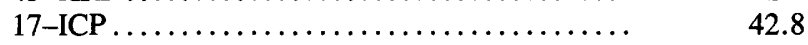

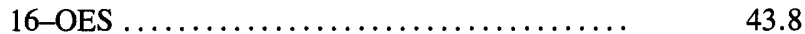

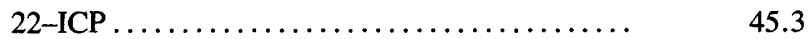

$14-\mathrm{XRF} \ldots \ldots \ldots \ldots \ldots \ldots \ldots \ldots \ldots \ldots \ldots \ldots \ldots \ldots$

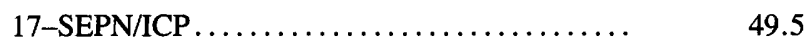

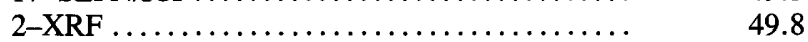

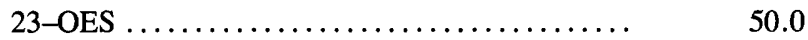

$15-$ OES ....................... 


\begin{tabular}{|c|c|}
\hline \multicolumn{2}{|l|}{ Yb data } \\
\hline $\begin{array}{l}\text { Lab code/ } \\
\text { method }\end{array}$ & $\begin{array}{c}\mu g / g \\
\text { in sample }\end{array}$ \\
\hline 26-SEPN/ICP $\ldots \ldots \ldots \ldots \ldots \ldots \ldots \ldots \ldots$ & 2.80 \\
\hline $55-N A A \ldots \ldots \ldots \ldots \ldots \ldots \ldots \ldots \ldots \ldots \ldots \ldots \ldots \ldots \ldots$ & 2.9 \\
\hline $60-N A A . \ldots \ldots \ldots \ldots \ldots \ldots \ldots \ldots \ldots \ldots \ldots \ldots \ldots \ldots \ldots$ & 2.9 \\
\hline $7-\mathrm{ICP} \ldots \ldots \ldots \ldots \ldots \ldots \ldots \ldots \ldots \ldots \ldots$ & 3.00 \\
\hline 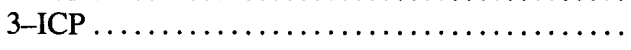 & 3.00 \\
\hline 23-ICP. & 3.00 \\
\hline $50-\mathrm{ICP} \ldots \ldots \ldots \ldots \ldots \ldots \ldots \ldots \ldots \ldots \ldots \ldots \ldots \ldots \ldots$ & 3.01 \\
\hline 24-SEPN/ICP $\ldots \ldots \ldots \ldots \ldots \ldots \ldots \ldots \ldots \ldots$ & 3.04 \\
\hline 63-ICPMS $\ldots \ldots \ldots \ldots \ldots \ldots \ldots \ldots \ldots$ & 3.04 \\
\hline $14-$ SEPN/ICP $\ldots \ldots \ldots \ldots \ldots \ldots \ldots \ldots \ldots$ & 3.08 \\
\hline $7-$ SEPN/ICP $\ldots \ldots \ldots \ldots \ldots \ldots \ldots \ldots \ldots \ldots$ & 3.10 \\
\hline $49-$ SEPN/ICP $\ldots \ldots \ldots \ldots \ldots \ldots \ldots \ldots \ldots \ldots \ldots \ldots$ & 3.11 \\
\hline $8-$ SEPN/ICP $\ldots \ldots \ldots \ldots \ldots \ldots \ldots \ldots \ldots$ & 3.15 \\
\hline 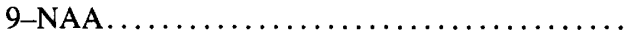 & 3.33 \\
\hline $10-N A A \ldots \ldots \ldots \ldots \ldots \ldots \ldots \ldots$ & 3.39 \\
\hline 7-NAA. & 3.40 \\
\hline $57-I C P M S \ldots \ldots \ldots \ldots \ldots \ldots \ldots \ldots \ldots$ & 3.40 \\
\hline $56-N A A . \ldots \ldots \ldots \ldots \ldots \ldots \ldots \ldots \ldots$ & 3.52 \\
\hline $17-\mathrm{ICP} \ldots \ldots \ldots \ldots \ldots \ldots \ldots \ldots \ldots$ & 3.55 \\
\hline 14-EXTR/ICP $\ldots \ldots \ldots \ldots \ldots \ldots \ldots \ldots$ & 3.80 \\
\hline 12-NAA. & 3.92 \\
\hline 15-NAA. & 4.30 \\
\hline$\ldots \ldots \ldots \ldots \ldots \ldots \ldots \ldots \ldots \ldots$ & 5.00 \\
\hline$\ldots \ldots \ldots \ldots \ldots \ldots \ldots \ldots \ldots$ & 5.05 \\
\hline 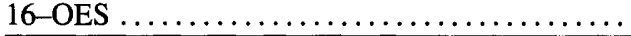 & 5.23 \\
\hline
\end{tabular}

\begin{tabular}{|c|c|}
\hline \multicolumn{2}{|l|}{ Zn data } \\
\hline $\begin{array}{l}\text { Lab code/ } \\
\text { method }\end{array}$ & $\begin{array}{c}\mu \mathrm{g} / \mathrm{g} \\
\text { in sample }\end{array}$ \\
\hline 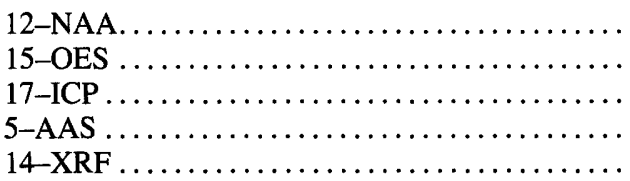 & $\begin{array}{r}<30 \\
50.0 \\
55.5 \\
55.8 \\
56.5\end{array}$ \\
\hline 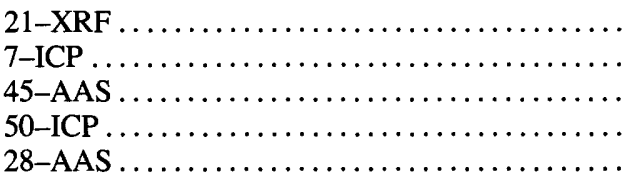 & $\begin{array}{l}57.0 \\
57.5 \\
59.3 \\
59.4 \\
60.0\end{array}$ \\
\hline 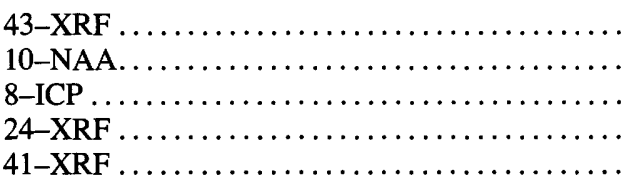 & $\begin{array}{l}60.3 \\
61.0 \\
62.5 \\
64.0 \\
64.3\end{array}$ \\
\hline 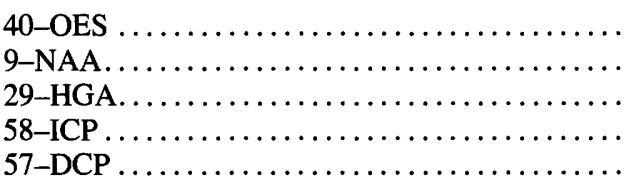 & $\begin{array}{l}65.0 \\
65.8 \\
67.0 \\
68.0 \\
70.0\end{array}$ \\
\hline 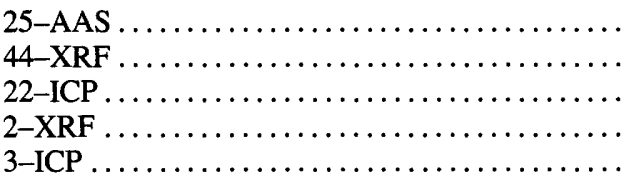 & $\begin{array}{l}70.5 \\
71.3 \\
71.5 \\
71.8 \\
72.0\end{array}$ \\
\hline 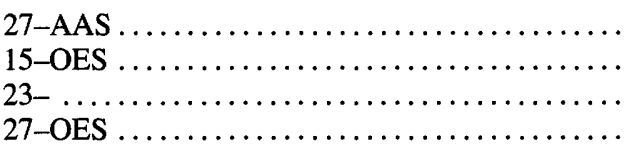 & $\begin{array}{r}72.0 \\
73.0 \\
76.0 \\
100\end{array}$ \\
\hline
\end{tabular}




\begin{tabular}{|c|c|}
\hline \multicolumn{2}{|l|}{$\mathrm{Zr}$ data } \\
\hline $\begin{array}{l}\text { Lab code/ } \\
\text { method }\end{array}$ & $\begin{array}{c}\mu \mathrm{g} / \mathrm{g} \\
\text { in sample }\end{array}$ \\
\hline 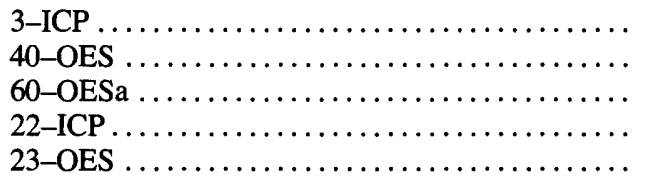 & $\begin{array}{l}101 \\
112 \\
130 \\
141 \\
150\end{array}$ \\
\hline 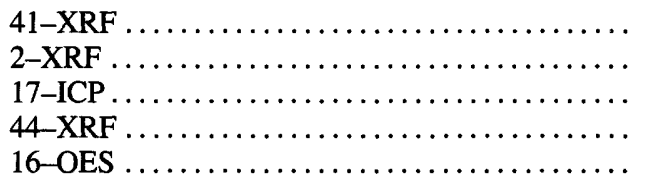 & $\begin{array}{l}153 \\
156 \\
159 \\
160 \\
160\end{array}$ \\
\hline 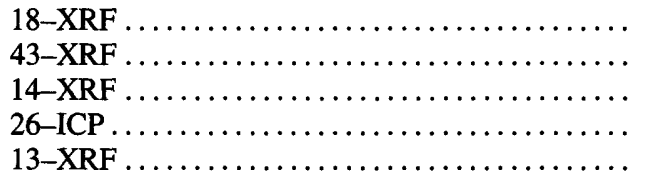 & $\begin{array}{l}163 \\
164 \\
167 \\
168 \\
169\end{array}$ \\
\hline 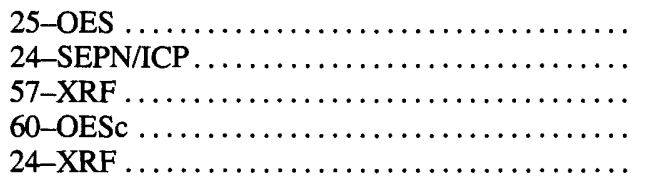 & $\begin{array}{l}170 \\
171 \\
172 \\
181 \\
191\end{array}$ \\
\hline 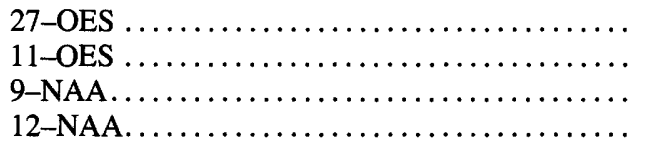 & $\begin{array}{l}230 \\
237 \\
318 \\
408\end{array}$ \\
\hline
\end{tabular}


Chapter B

Determination of Major and 11 Trace Elements (XRF), Gold (AAS), Carbon and Sulfur (COUL) in USGS Devonian Ohio Shale SDO-1

By OTMAR SPIES, BERNHARD STRIBRNY, JOSEF KONOPASEK, and HANS URBAN

U.S. GEOLOGICAL SURVEY BULLETIN 2046

THE USGS REFERENCE SAMPLE DEVONIAN OHIO SHALE SDO-1 



\title{
CONTENTS
}

\author{
Abstract B1 \\ Introduction B1 \\ Experiment Parameters B1 \\ Determination of Major and Trace Elements by X-ray Fluorescence \\ Analysis B1 \\ Sample Preparation B1 \\ Instrumentation B3 \\ Data Evaluation B3 \\ Loss on Ignition $\mathbf{B} 4$ \\ Determination of Gold by Graphite Furnace Atomic Absorption \\ Spectrometry B4 \\ Determination of CARBON total $, \mathrm{CARBON}_{\text {carbonate }}, \mathrm{CARBON}_{\text {organic }}$, and \\ Conclusions B5 \\ SULFUR by Coulometric Titration B5 \\ References B6
}

\section{FIGURES}

1. Flow diagram of analytical sequence for the analysis of SDO-1 B2

2. Recommended average values for SDO-1 and their ranges of confidence in comparison to the mean values as reported in tables 3 and 4 B6

\section{TABLES}

1. Instrument set-up (Rh-tube) of major and trace element analyses $\mathbf{B 3}$

2. The program parameters for the HGA-400 B4

3. XRF determinations of major and trace elements in USGS Devonian Ohio Shale SDO-1 B4

4. Coulometric determinations of $\mathrm{CARBON}_{\text {total }}, \mathrm{CARBON}_{\text {carbonate }}, \mathrm{CARBON}_{\text {organic }}$ and SULFUR in USGS Devonian Ohio Shale SDO-1 B5

5. Results for gold after fire assay preconcentration $\mathbf{B 5}$ 



\title{
Determination of Major and 11 Trace Elements (XRF), Gold (AAS), Carbon and Sulfur (COUL) in USGS Devonian Ohio Shale SDO-1
}

\author{
By Otmar Spies, Bernhard Stribrny, Josef Konopasek, and Hans Urban ${ }^{1}$
}

\begin{abstract}
An X-ray fluorescence (XRF) analytical program for the determination of 10 major and 11 trace elements in rock samples has been developed using a glass bead 32 $\mathrm{mm}$ in diameter. The method was applied to the characterization of the shale SDO-1 as a reference sample in conjunction with the Metalliferous Black Shales Project of the International Geologic Congress. Additionally, loss on ignition and carbon and sulfur, using coulometry (COUL), were determined as major constituents in SDO-1; and trace gold was determined by graphite furnace atomic absorption spectrometry (AAS) after fire assay preconcentration. The data were used in establishing recommended concentrations for constituents in the reference sample.
\end{abstract}

\section{INTRODUCTION}

As a contribution to the International Geologic Congress Project (IGCP) 254, "Metalliferous Black Shales," our working group investigated black-shale-hosted copper, gold, and lead-zinc deposits in the Northeastern Rhenish Massif of central Germany (Stribrny, 1987; Stribrny and others, 1988). These deposits occur in the Lower Carboniferous Variscan folded black shale series composed of black shales, black lydites, siliceous limestones, and clay shales. Commonly, these rocks host low-grade concentrations of stratabound sulfides, which are interpreted as sedimentary-exhalative depositions. Intraformational mobilizations and migrations of metals and their reprecipitation in physico-chemical traps led locally to minable ores. Additional metal concentrations were related to supergene processes (Stribrny and Urban, 1989).

\footnotetext{
Manuscript approved for publication September 15, 1992.

${ }^{1}$ Frankfurt University
}

\section{EXPERIMENT PARAMETERS}

\section{Determination of Major and Trace Elements by $\mathrm{X}$-ray Fluorescence Analysis}

An X-ray fluorescence (XRF) analytical program (called GEO, fig. 1) for the determination of 10 major and 11 trace elements in rock samples has been developed using a glass bead $32 \mathrm{~mm}$ in diameter. Fusions of rock samples were made using a flux-to-sample ratio of $4: 1$. Calibration was established with 41 international geochemical reference standards.

\section{Sample Preparation}

For the sample preparation, a fusion method has been adopted. First, the rock powders were dried at $105^{\circ} \mathrm{C}$ for 3 hours, and the flux (Merck A12 Spectromelt $=66$ percent $\mathrm{Li}_{2} \mathrm{~B}_{4} \mathrm{O}_{7}+34$-percent $\mathrm{LiBO}_{2}$ ) was heated at $450^{\circ} \mathrm{C}$ for 3 hours. Samples containing nonoxides as sulfides and organic carbon required further pretreatment prior to the fusion. For this pretreatment, $1.5 \mathrm{~g} \mathrm{NH}_{4} \mathrm{NO}_{3}$ was added to the mixture of $1 \mathrm{~g}$ rock powder and $4 \mathrm{~g}$ Spectromelt. Heating the Pt-Au crucible at $450^{\circ} \mathrm{C}$ for 1 hour disassociated the $\mathrm{NH}_{4} \mathrm{NO}_{3}$ with subsequent oxidation of nonoxides. After oxidation, $20 \mathrm{mg} \mathrm{NH}_{4} \mathrm{Br}$ was added before proceeding with the fusions to avoid adhesion and erosion of the nonwetting Pt-Au alloy crucible.

The samples were then mixed with flux at a $4: 1$ flux-to-sample ratio by weight. The mixtures were fused at $1,200^{\circ} \mathrm{C}$ for 6 minutes in the Pt-Au crucible utilizing a RF induction heater with mechanical agitation (type: KontronRotomelt). At the end of fusion the crucible was inverted, and the melt was cast into a polished mold held at $500^{\circ} \mathrm{C}$. The mold was then removed from the hot plate and cooled to room temperature. 


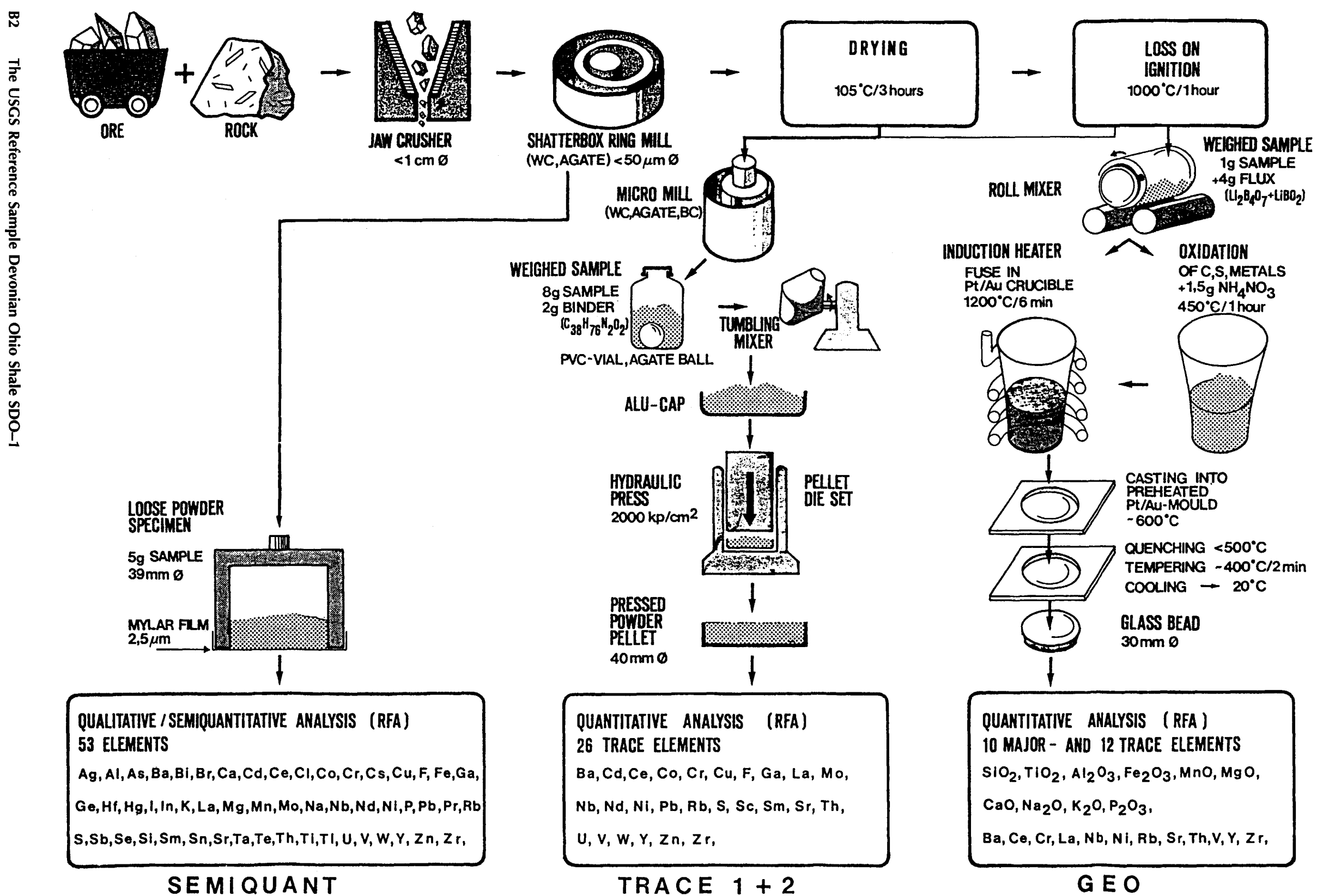

Figure 1. Flow diagram of analytical sequence for the analysis of SDO-1. 
Table 1. Instrument set-up (Rh-tube) of major and trace element analyses

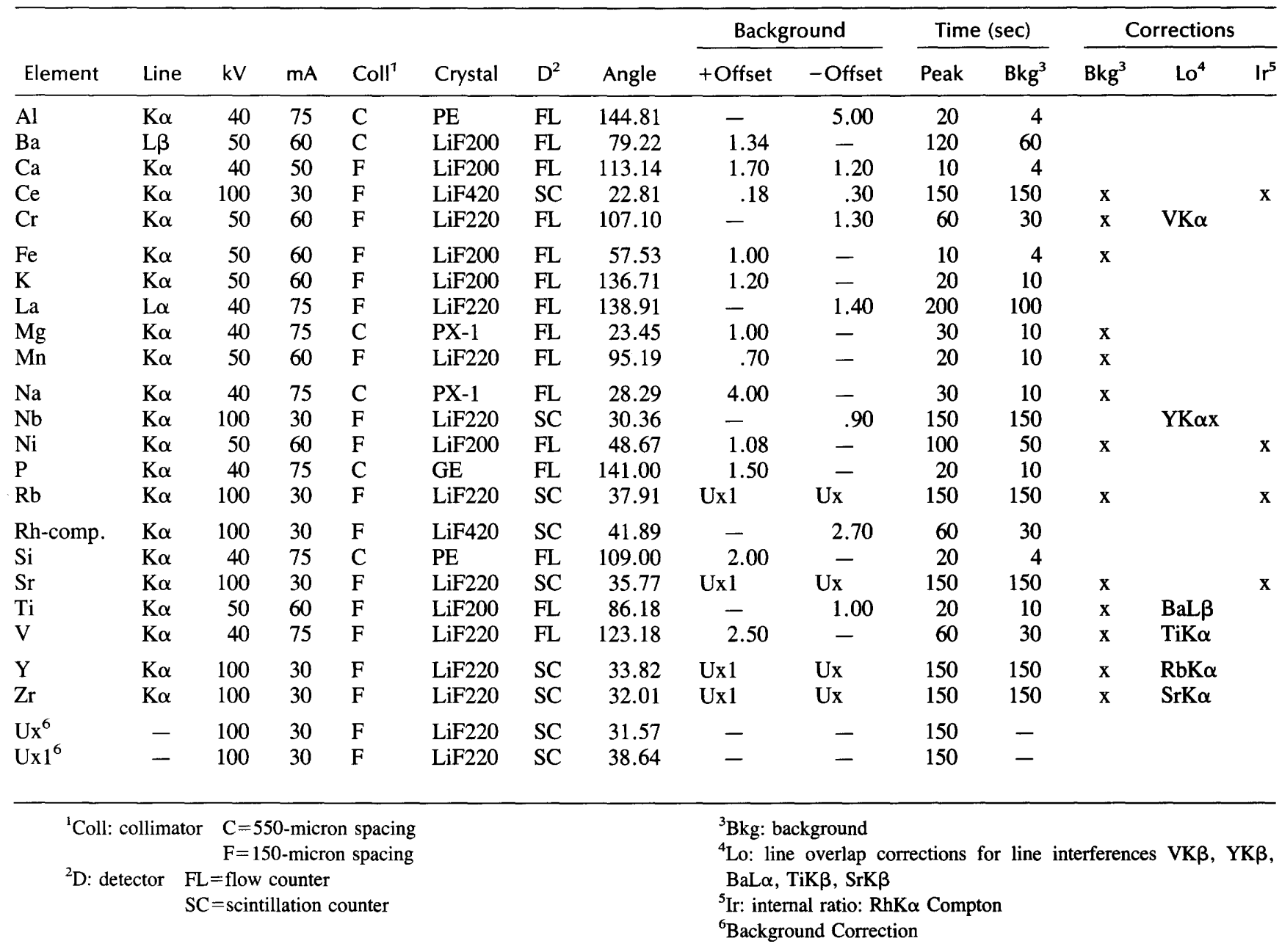

\section{Instrumentation}

Analyses were performed on an automated Philips PW 1404 sequential X-ray spectrometer controlled by a DEC micro-PDP11. The PW 1404 is equipped with a $100-\mathrm{kV}(3-\mathrm{kW})$ generator and a 12-position sample changer. A rhodium side-window tube and LiF420, LiF220, LiF200, GE, PE, and PX-1 analyzing crystals were used for the determinations (table 1).

Correction of the quantitative measurement data to compensate for drift was done using a monitor sample. A polished shoshonitic volcanic rock was prepared as monitor sample, and eight major elements ( $\mathrm{Al}, \mathrm{Ca}, \mathrm{Fe}, \mathrm{K}, \mathrm{Mg}, \mathrm{Na}$, $\mathrm{Si}, \mathrm{Ti}$ ) in the analytical program were corrected with drift-correction factors.

\section{Data Evaluation}

For matrix correction the new Philips calculation model was applied. The Philips model is a combination of correction models (Claisse-Quintin, 1967; de Jongh, 1973;
Lucas-Tooth-Pyne, 1964; and Rasberry-Heinrich, 1974). The corrections may be based on count rates, concentrations, or combinations of both. The Philips model includes terms that can be calculated or entered during the regression analyses for spectral interferences, constant background, varying background, and line overlap correction factors.

In the first step of regression analyses, theoretical alphas were used based on the fundamental parameter method (Criss and Birks, 1968; Shiraiwa and Fujino, 1966). The sample matrix, spectrometer geometry, and X-ray tube are taken into account in these theoretical alpha values. Regression analysis is considered to be more general and flexible for application to samples with wide concentration ranges. During regression analysis, the matrix correction factors (theoretical alphas) are, in part, recalculated.

For several elements ( $\mathrm{Ni}, \mathrm{Rb}, \mathrm{Sr}, \mathrm{Y}, \mathrm{Zr}, \mathrm{Nb}, \mathrm{Ce})$ the "internal ratio" method has been applied (Leoni and Saitta, 1977). This matrix correction is based on RhK $\alpha$ Compton scatter and corrects for absorption effects due to the major elements without the need to determine these elements (table 1). 


\section{Loss on Ignition}

The rock powders were dried in an oven for 3 hours at $105^{\circ} \mathrm{C}$. The dried rock powders were ignited for 1 hour at $1,000^{\circ} \mathrm{C}$ in a muffle furnace using platinum crucible. Loss on ignition (LOI) was determined in duplicate by igniting $1-\mathrm{g}$ aliquots of sample powders.

\section{Determination of Gold by Graphite Furnace Atomic Absorption Spectrometry}

The determination of gold in geological samples requires its separation from the matrix. Instead of the frequently used 4-methyl-2-pentanon, the gold with palladium was extracted quantitatively by dibutyl sulfide in toluene, following a method described by Rubeska and others (1977). Besides $\mathrm{Au}$ and Pd, some Ag will also be extracted; whereas extraction of other elements can be neglected. A repeated treatment of geological samples with
Table 2. The program parameters for the HGA-400

\begin{tabular}{|c|c|c|c|c|c|}
\hline \multicolumn{2}{|c|}{ Step } & 1 & 2 & 3 & 4 \\
\hline Temperature & ${ }^{\circ} \mathrm{C}$ & 120 & 650 & 1900 & 2650 \\
\hline Ramp time & $\mathrm{sec}$ & 10 & 10 & 0 & 1 \\
\hline Hold time & $\mathrm{sec}$ & 20 & 30 & 5 & 3 \\
\hline Gas flow & $\mathrm{mL} / \mathrm{min}$ & & & 50 & \\
\hline
\end{tabular}

aqua regia gives 99 percent recovery of Au. Gold contents up to $0.5 \mu \mathrm{g} / \mathrm{g}$ should be detected by graphite furnace atomic absorption spectrometry (AAS), whereas higher contents demand flame AAS. Two portions of $10 \mathrm{~g}$ were taken from each bottle (bottles no. 0521 and no. 0756). The samples were dried at $110^{\circ} \mathrm{C}$ and then ashed for 2 hours each first at $480^{\circ} \mathrm{C}$ and then at $600^{\circ} \mathrm{C}$, to oxidize all organic material, carbon, and sulfur. For total decomposition the samples were dissolved by $\mathrm{HF}$ and $\mathrm{HCLO}_{4}$ and then treated with aqua regia. Gold was extracted from the solutions using $0.2 \mathrm{M}$ dibutyl sulfide in toluene. The measurements

Table 3. X-ray fluorescence (XRF) determinations of major and trace elements in USGS Devonian Ohio Shale SDO-1

\begin{tabular}{|c|c|c|c|c|c|c|c|c|c|c|c|c|}
\hline \multirow[b]{2}{*}{$\begin{array}{c}\text { Element } \\
(w t \%)\end{array}$} & \multicolumn{3}{|c|}{$\begin{array}{l}\text { SDO-1 } \\
\text { B. No. } 0521\end{array}$} & \multicolumn{3}{|c|}{$\begin{array}{c}\text { SDO-1 } \\
\text { B. No. } 0756\end{array}$} & \multicolumn{3}{|c|}{$\begin{array}{c}\text { SDO-1 } \\
\text { B. No. } 0310\end{array}$} & \multicolumn{3}{|c|}{$\begin{array}{l}\text { SDO-1 } \\
\text { Mean }\end{array}$} \\
\hline & $\underset{(n=6)}{X}$ & $\begin{array}{c}\mathrm{RMS}^{1} \\
(\%)\end{array}$ & $\begin{array}{c}\mathrm{RMS}^{2} \\
\text { (\% rel.) }\end{array}$ & $\underset{(n=6)}{\mathrm{X}}$ & $\begin{array}{c}\mathrm{RMS}^{1} \\
(\%)\end{array}$ & $\begin{array}{c}\mathrm{RMS}^{2} \\
\text { (\% rel.) }\end{array}$ & $\underset{(n=6)}{\mathrm{X}}$ & $\begin{array}{c}\mathrm{RMS}^{\top} \\
(\%)\end{array}$ & $\begin{array}{c}\mathrm{RMS}^{2} \\
\text { (\% rel.) }\end{array}$ & $\underset{(n=6)}{\mathrm{X}}$ & $\begin{array}{c}\mathrm{RMS}^{1} \\
(\%)\end{array}$ & $\begin{array}{c}\mathrm{RMS}^{2} \\
\text { (\% rel.) }\end{array}$ \\
\hline $\mathrm{SiO}_{2}$ & 49.82 & 0.063 & 0.13 & 48.19 & 0.057 & 0.12 & 48.61 & 0.055 & 0.11 & 48.88 & 0.714 & 1.46 \\
\hline $\mathrm{TiO}_{2}$ & .69 & .001 & .13 & .67 & .003 & .49 & .68 & .004 & .64 & .68 & .011 & 1.62 \\
\hline $\mathrm{Al}_{2} \mathrm{O}_{3}$ & 12.54 & .032 & .25 & 12.15 & .026 & .22 & 12.51 & .031 & .25 & 12.40 & .186 & 1.50 \\
\hline $\mathrm{Fe}_{2} \mathrm{O}_{3 \text { Tот }}$ & 9.31 & .012 & .13 & 8.95 & .012 & .13 & 9.12 & .007 & .08 & 9.12 & .150 & 1.65 \\
\hline $\mathrm{MnO}$ & .039 & .001 & 3.59 & .035 & .002 & 4.50 & .037 & .001 & 2.75 & .037 & .020 & 5.56 \\
\hline $\mathrm{MgO}$ & 1.53 & .020 & 1.28 & 1.49 & .008 & .53 & 1.50 & .019 & 1.28 & 1.51 & .024 & 1.58 \\
\hline $\mathrm{CaO}$ & 1.07 & .008 & .78 & 1.03 & .009 & .92 & 1.04 & .006 & .58 & 1.05 & .018 & 1.72 \\
\hline $\mathrm{Na}_{2} \mathrm{O}$ & .44 & .014 & 3.23 & .45 & .014 & 3.15 & .31 & .018 & 5.89 & .40 & .067 & 16.79 \\
\hline $\mathrm{K}_{2} \mathrm{O}$ & 3.25 & .019 & .58 & 3.13 & .009 & .29 & 3.17 & .011 & .36 & 3.17 & .054 & 1.67 \\
\hline $\mathrm{P}_{2} \mathrm{O}_{5}$ & .11 & .003 & 2.74 & .11 & .002 & 2.18 & .11 & .002 & 1.89 & .11 & .003 & 2.38 \\
\hline LOI & 19.77 & & & 21.75 & & & 20.98 & & & 20.83 & & \\
\hline \multirow[t]{2}{*}{ Total } & 98.57 & & & 97.96 & & & 98.07 & & & 98.19 & & \\
\hline & $(\mu g / g)$ & $(\mu \mathrm{g} / \mathrm{g})$ & & $(\mu g / g)$ & $(\mu \mathrm{g} / \mathrm{g})$ & & $(\mu g / g)$ & $(\mu g / g)$ & & $(\mu g / g)$ & $(\mu \mathrm{g} / \mathrm{g})$ & \\
\hline V & 162 & 3 & 2.0 & 156 & 4 & 2.5 & 156 & 5 & 3.3 & 158 & 5 & 3.0 \\
\hline $\mathrm{Cr}$ & 63 & 3 & 4.5 & 65 & 6 & 8.7 & 66 & 5 & 7.0 & 65 & 5 & 6.8 \\
\hline $\mathrm{Ni}$ & 93 & 2 & 2.2 & 92 & 2 & 1.5 & 94 & 2 & 2.0 & 93 & 2 & 2.2 \\
\hline $\mathrm{Rb}$ & 135 & 1 & .6 & 131 & 1 & .5 & 136 & 1 & .5 & 134 & 3 & 1.7 \\
\hline $\mathrm{Sr}$ & 84 & 1 & 1.2 & 81 & 1 & 1.2 & 54 & 1 & 1.8 & 73 & 14 & 18.6 \\
\hline$Y$ & 47 & 1 & 1.2 & 47 & 1 & 2.1 & 29 & 1 & 2.4 & 41 & 9 & 21.7 \\
\hline $\mathrm{Zr}$ & 175 & 1 & .3 & 170 & 1 & .3 & 167 & 1 & .42 & 171 & 3 & 1.6 \\
\hline $\mathrm{Nb}$ & 13 & 1 & 4.9 & 13 & 1 & 3.4 & 13 & 1 & 1.4 & 13 & 1 & 4.7 \\
\hline $\mathrm{Ba}$ & 389 & 29 & 7.6 & 380 & 13 & 3.4 & 357 & 17 & 4.6 & 375 & 24 & 6.2 \\
\hline $\mathrm{La}$ & 37 & 10 & 26.0 & 34 & 4 & 9.8 & 37 & 6 & 14.9 & 36 & 7 & 18.0 \\
\hline $\mathrm{Ce}$ & 84 & 20 & 24.4 & 71 & 7 & 10.3 & 92 & 12 & 12.6 & 82 & 16 & 19.4 \\
\hline
\end{tabular}

${ }^{1}$ RMS: root mean square (standard deviation)

${ }^{2}$ RMS (\% rel): percentage relative root mean square 
Table 4. Coulometric determinations of $\mathrm{C}_{\mathrm{tot}}, \mathrm{C}_{\mathrm{carb}}, \mathrm{C}_{\mathrm{org}}$, and $\mathrm{S}$ in USGS Devonian Ohio Shale SDO-1

\begin{tabular}{|c|c|c|c|c|c|c|c|c|c|c|c|c|}
\hline \multirow[b]{2}{*}{$\begin{array}{c}\text { Element } \\
(w t \%)\end{array}$} & \multicolumn{3}{|c|}{$\begin{array}{c}\text { SDO-1 } \\
\text { B. No. } 0521\end{array}$} & \multicolumn{3}{|c|}{$\begin{array}{c}\text { SDO-1 } \\
\text { B. No. } 0756\end{array}$} & \multicolumn{3}{|c|}{$\begin{array}{c}\text { SDO-1 } \\
\text { B. No. } 0310\end{array}$} & \multicolumn{3}{|c|}{$\begin{array}{l}\text { SDO-1 } \\
\text { Mean }\end{array}$} \\
\hline & $\underset{(n=6)}{X}$ & $\begin{array}{c}\mathrm{RMS}^{1} \\
(\%)\end{array}$ & $\begin{array}{c}\mathrm{RMS}^{2} \\
\text { (\% rel.) }\end{array}$ & $\underset{(n=6)}{X}$ & $\begin{array}{c}\mathrm{RMS}^{1} \\
(\%)\end{array}$ & $\begin{array}{c}\mathrm{RMS}^{2} \\
\text { (\% rel.) }\end{array}$ & $\underset{(n=6)}{X}$ & $\begin{array}{c}\mathrm{RMS}^{1} \\
(\%)\end{array}$ & $\begin{array}{l}\mathrm{RMS}^{2} \\
\text { (\% rel.) }\end{array}$ & $\underset{(n=6)}{X}$ & $\begin{array}{c}\mathrm{RMS}^{1} \\
(\%)\end{array}$ & $\begin{array}{c}\mathrm{RMS}^{2} \\
\text { (\% rel.) }\end{array}$ \\
\hline$C_{\text {tot }}$ & 10.34 & 0.061 & 0.59 & 9.70 & 0.025 & 0.26 & 9.97 & 0.086 & 0.86 & 10.03 & 0.288 & 2.87 \\
\hline $\mathrm{C}_{\text {carb }}$ & .30 & .013 & 4.33 & .15 & .004 & 2.67 & .23 & .004 & 1.74 & .23 & .066 & 28.70 \\
\hline $\mathrm{C}_{\text {org }}$ & $\begin{array}{l}10.04 \\
(n=5)\end{array}$ & .063 & .63 & $\begin{array}{c}9.55 \\
(n=4)\end{array}$ & .026 & .27 & $\begin{array}{c}9.74 \\
(n=9)\end{array}$ & .090 & .92 & $\begin{array}{c}9.80 \\
(n=18)\end{array}$ & .226 & 2.31 \\
\hline$S$ & 5.23 & .071 & 1.36 & 5.10 & .022 & .43 & 5.18 & .016 & .31 & 5.17 & .062 & 1.20 \\
\hline
\end{tabular}

${ }^{1} \mathrm{RMS}$ : root mean square (standard deviation)

${ }^{2} \mathrm{RMS}$ (\% rel): percentage relative root mean square

Table 5. Results for gold after fire assay preconcentration

\begin{tabular}{cccr}
\hline 10-g run & Au $(\mu \mathrm{g} / \mathrm{g})$ & $25-\mathrm{g}$ run & $\mathrm{Au}(\mu \mathrm{g} / \mathrm{g})$ \\
\hline SDO-1 bottle no. 0521 & $<0.005$ & SDO-1 bottle no. 0521 & 0.004 \\
& .01 & SDO-1 bottle no. 0756 & $<.003$ \\
SDO-1 bottle no. 0756 & $<.005$ & & \\
& .008 & & \\
\hline
\end{tabular}

were made using a Perkin Elmer HGA-400 graphite furnace in combination with a Zeiss FMD 4 atomic absorption spectrometer. The program parameters for the HGA, from Konopasek and others (1988), are given in table 2.

\section{Determination of CARBON CARBON $_{\text {carbonate }}$ CARBON $_{\text {organic, }}$ and SULFUR by Coulometric Titration}

The carbon analyses were made following the method of Herrmann and Knake (1973). The sulfur determinations were done by adopting the method published by Lange and Brumsack (1977). For each run, portions of $100 \mathrm{~g}$ were taken from the bottles no. 0521 , no. 0756 , and no. 0310 . To measure the total carbon content, $100 \mathrm{mg}$ of each sample were ignited without additional reagents in an oxygen current at about $1,250^{\circ} \mathrm{C}$. The carbonate carbon content was determined by dissolving $100 \mathrm{mg}$ of each sample in orthophosphoric acid and measuring the evolved $\mathrm{CO}_{2}$. The organic carbon content was calculated by difference.

To measure the sulfur content, $100 \mathrm{mg}$ of each sample were mixed with $500 \mathrm{mg}$ of $\mathrm{V}_{2} \mathrm{O}_{5}$ and $500 \mathrm{mg}$ of $\mathrm{Fe}$ and ignited at $1,380^{\circ} \mathrm{C}$. The analyses were performed on a Ströhlein Coulomat 702/SO/CS/E. The $\mathrm{SO}_{2}$ released is absorbed in a $\mathrm{Na}_{2} \mathrm{SO}_{4}$ solution $\left(10 \mathrm{~g} \mathrm{Na}_{2} \mathrm{SO}_{4}\right.$ in $200-\mathrm{mL}$ doubly distilled water to which $2 \mathrm{~mL}$ of 30 -percent $\mathrm{H}_{2} \mathrm{O}_{2}$ had been added), lowering its $\mathrm{pH}$. Again the $\mathrm{pH}$ is returned to its starting point by electrolysis. The amount of energy required is equivalent to the quantity of $\mathrm{SO}_{2}$ absorbed.

\section{CONCLUSIONS}

Analytical data from all XRF determinations are presented in table 3 , and analytical data for coulometric titrations to determine $\mathrm{C}$ and $\mathrm{S}$ are presented in table 4 . Gold data in table 5 show inhomogeneous distribution of gold in 10-g samples. Because only a single repeat analysis from each bottle at 25 -g sample sizes was possible, homogeneity could not be assessed based on the larger sample weight. These results also appear in the table.

Precisions for $\mathrm{MnO}, \mathrm{Na}_{2} \mathrm{O}$, and $\mathrm{P}_{2} \mathrm{O}_{5}$ within bottle are larger than expected based on past experience with the method. As a result, between-bottle analysis of variance shows no significant difference despite suspected inhomogeneity cited in Kane and others (1990). Similar inability to assess material in inhomogeneity because of large withinbottle imprecision affects the measurement for several trace elements $(\mathrm{Cr}, \mathrm{Sr}, \mathrm{Y}, \mathrm{Ba}, \mathrm{La}$, and $\mathrm{Ce})$.

As figure 2 shows, the measured mean values $(n=18)$ presented in table 2 fit well within the range of confidence ( \pm standard deviations) of the recommended average values for SDO-1 published by Kane and others (1990). Mean values of $\mathrm{Fe}_{2} \mathrm{O}_{3}, \mathrm{~K}_{2} \mathrm{O}, \mathrm{Nb}$, and $\mathrm{Rb}$ do not plot exactly within the confidence range shown; however, expansion of that range to \pm two standard deviations does encompass those values (fig. 1); therefore, it can be concluded that the analytical methods (XRF, AAS, and coulometry) used are satisfactory, reliable tools for solving analytical problems related to the geochemistry of black shales. 


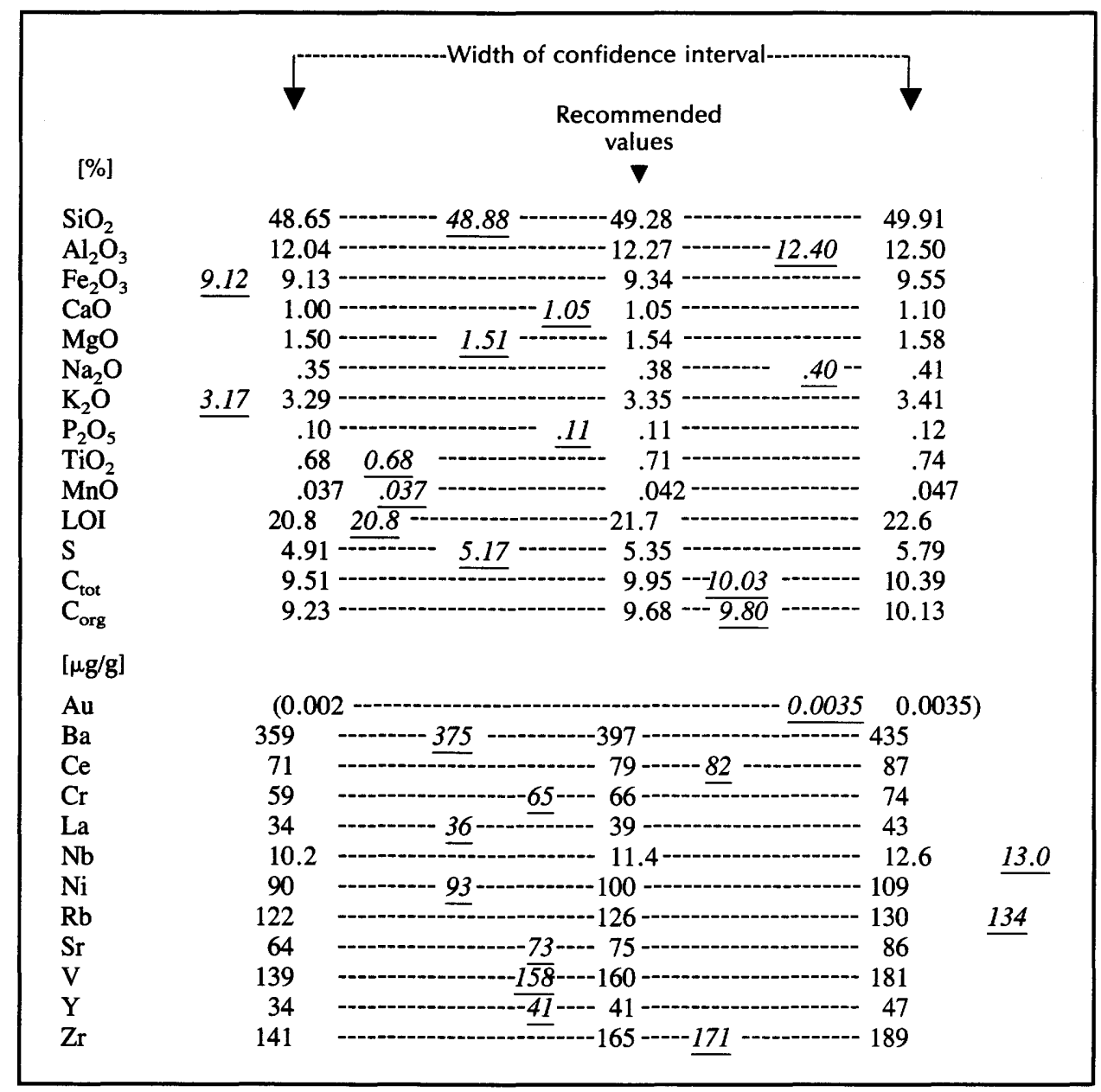

Figure 2. Recommended average values for SDO-1 (central column) and their ranges of confidence ( \pm standard deviation) (Kane and others, 1990) in comparison to the mean values as reported in tables 3 and 4 (italicized and underscored).

\section{REFERENCES}

Claisse, Ferdinand, and Quintin, M., 1967, Generalization of the Lachance-Trail method for the correction of the matrix effect in X-ray fluorescence analysis: Canadian Spectroscopy, $v$. $12,129 \mathrm{p}$.

Criss, J.W., and Birks, L.S., 1968, Calculation methods for fluorescent $X$-ray spectrometry-empirical coefficients vs. fundamental parameters: Analytical Chemistry, v. 46, 81 p.

de Jongh, W.K., 1973, X-ray fluorescence analysis applying theoretical matrix corrections-stainless steel: X-ray Spectrometry, v. 2, p. 151.

Herrmann, A.G., and Knake, Doris, 1973, Coulometrisches Verfahren zur Bestimmung von gesamt-, carbonat- und nichtcarbonat-Kohlenstoff in magmatischen, metamorphen und sedimentären Gesteinen: Zeitschrift für Analytische Chemie, v. 266, p. 196-201.

Kane, J.S., Arbogast, B.F., and Leventhal, J.S., 1990, Characterization of Devonian Ohio Shale SDO-1 as a USGS geochemical reference sample: Geostandards Newsletter, v. 14, p. $169-196$.
Kikkert, J.N., 1981, Accurate geochemical analysis of samples of unknown composition, 31st Annual Denver X-ray Conference, $12 \mathrm{p}$.

Konopasek Josef, Zereini, Fathi, and Urban, Hans, 1988, GoldBestimmung in geologischen Proben: Institute of Geochemistry, Frankfurt University, Open-File Report 1988-3, 1-11.

Lange, J., and Brumsack, H.J., 1977, Total sulfur analysis in geological and biological materials by coulometric titration following combustion: Zeitschrift für Analytische Chemie, v. 286 , p. $361-366$.

Leoni, Leonardo, and Saitta, Maurizio, 1977, Matrix effect corrections by $\mathrm{Ag} \mathrm{K} \alpha$ Compton scattered ratiation in the analysis of rock samples for trace elements: X-ray Spectrometry, v. 6, p. 181.

Lucas-Tooth, H.J., and Pyne, C., 1964, The accurate determination of major constituents by X-ray fluorescent analysis in the presence of large interelement effects: Advances in $\mathrm{X}$-ray Analysis, v. 7, 523 p.

Rasberry, S.D., and Heinrich, K.F.J., 1974, Calibration for interelement effects in X-ray fluorescence analysis: Analytical Chemistry, v. 46, 81 p. 
Rubeska, Ivan, Koreckova, J., and Weiss, Dalibor, 1977, The determination of gold and palladium in geological materials by atomic absorption after extraction with dibutyl sulfide: Atomic Absorption Newsletter, v. 16, 1-3.

Shiraiwa, T., and Fujino, N., 1966, Japan Journal of Applied Physics, v. 5, p. 886.

Stribrny, Bernhard, 1987, Die kupfererzlagerstätte Marsberg im rheinischen Schiefergebirge-Rückblick und Stand der Forschung: Erzmetall, 40, 7/8, p. 423-427.
Stribrny, Bernhard, and Urban, Hans, 1989, Lagerstättenbildende, supergene umlagerungsprozesse in Schwarzschiefern: in Weisser, J.D. et al., eds.: Supergene Lagerstätten und ihre rohstoffwirtschaftliche Bedeutung: Erzmetall, 42, 7/8, p. 348-350.

Stribrny, Bernhard, Urban, Hans, and Webor, Herrbert, 1988, The lower Carboni Ferous Black Shale Formation, a possible source fo noble and base metal deposits in the Northeastern Rhenish Massif, FR Germany: Mineralogy and Petrology, 39 , p. $129-143$. 

Chapter C

Inductively Coupled Plasma Determination of Nine Rare Earth Elements in the USGS Devonian Ohio Shale SDO-1

By IWAN ROELANDTS

U.S. GEOLOGICAL SURVEY BULLETIN 2046

THE USGS REFERENCE SAMPLE DEVONIAN OHIO SHALE SDO-1 



\title{
CONTENTS
}

\author{
Abstract C1 \\ Introduction $\mathbf{C 1}$ \\ Experiment Parameters C1 \\ Apparatus C1 \\ ICP Equipment $\mathbf{C 1}$ \\ Cation-Exchange Columns C2 \\ Reagents C2 \\ Cation-Exchange Resin C2 \\ Rare Earth Element Standard Solutions C2 \\ Chemicals C2 \\ Analytical Procedure C2 \\ Dissolution of the Samples C2 \\ Cation-Exchange Separation C2 \\ ICP-AES Measurements C2 \\ Results and Discussion C2 \\ Acknowledgments C5 \\ References C5
}

\section{FIGURES}

1. Wavelength scan of the Sm 359.260-nanometer line C3

2. Chondrite-normalization pattern for the Devonian Ohio Shale SDO-1 C4

\section{TABLES}

1. Spectral interference correction coefficients C2

2. Determination of nine rare earth elements in USGS Devonian Ohio Shale SDO-1 C3

3. One-way analysis of variance $\mathbf{C 3}$

4. Results obtained for USGS Devonian Ohio Shale SDO-1 in the present work compared with previous ICP data $\mathbf{C 4}$

5. Determination of nine rare earth elements in USGS sedimentary reference samples $\mathbf{C 4}$ 


\title{
Inductively Coupled Plasma Determination of Nine Rare Earth Elements in the USGS Devonian Ohio Shale SDO-1
}

\author{
By Iwan Roelandts ${ }^{1}$
}

\begin{abstract}
The U.S. Geological Survey Devonian Ohio Shale SDO-1 has been analyzed for nine of the rare earth elements (REE) by inductively coupled argon plasma atomic emission spectrometry (ICP-AES). Triplicate portions from two bottles of the material were analyzed in random order to estimate analytical precision and test for homogeneity. The precision, based on the samples analyzed, is generally better than 1 percent. The one-way analysis of variance was used with our ICP determinations; no evidence for differences in REE concentrations between these two randomly selected bottles was observed.
\end{abstract}

\section{INTRODUCTION}

Nine of the rare earth elements (REE) in the U.S. Geological Survey (USGS) Devonian Ohio Shale SDO-1 were determined by inductively coupled plasma atomic emission spectrometry (ICP-AES) following cationexchange separation. Two bottles randomly selected from the stock were received and three separate portions from each bottle were analyzed to provide a one-way experimental design using the two bottles as the single variable of classification. The six portions were analyzed in random order.

The REE have been found to be extremely useful in several studies of sedimentary processes (weathering, transport, and diagenesis). Very few years ago, instrumental neutron activation analysis (INAA) and isotope dilution mass spectrometry (IDMS) were the only sensitive and accurate techniques in use for geochemical REE analysis. ICP-AES is rapidly becoming an established analytical method for the determination of REE, especially when large numbers of samples of common silicate rocks must be analyzed. This technique has several advantages, the prin-

Manuscript approved for publication September 15, 1992.

${ }^{1}$ University of Liege cipal of which are low detection limits, good precision and accuracy, low sample matrix effects, speed of analysis, and wide linear dynamic concentration range.

ICP spectra of rock samples are not simple; a very large number of emission lines are present. To avoid interference from other elements and thereby improve accuracy and detection limits, some workers chemically separated the REE as a group from the rock matrix, in most cases by an ion-exchange procedure (for example, Walsh and others, 1981; Brenner and others, 1981, 1984; Crock and Lichte, 1982; Bolton and others, 1983; Yoshida and others, 1983; Crock and others, 1984, 1986; Aulis and others, 1985; Jarvis and Jarvis, 1985, 1988; Roelandts and Michel, 1986; Roelandts, 1988a; Roelandts, 1990; Govindaraju and Mevelle, 1987; Zachman, 1988; Sulcek and others, 1989; Watkins and Nolan, 1990).

In this work a separation of the REE from the other constituents after an acid decomposition of the sample was achieved using the strong acid-type resin Dowex 50WX8. The REE were first held quantitatively on the cationic resin during the initial washing with $1.75 M$ hydrochloric acid to remove unwanted elements and then desorbed using $8 M$ nitric acid as an eluent. The experimental conditions of this separation procedure have been previously determined (Roelandts, unpub. data, 1975).

\section{EXPERIMENT PARAMETERS}

\section{Apparatus \\ ICP Equipment}

A Bausch and Lomb model 3510 sequential ICP spectrometer (Applied Research Laboratories) operated at 1.2 incident $\mathrm{kW}$ was used for this study. A DEC PDP-11 computer controlled all instrument functions. The plasma gas-flow and the coolant gas-flow rates were 0.8 and 12.0 $\mathrm{L} / \mathrm{min}$, respectively. A concentric Meinhard type nebulizer was employed, and the sample solution uptake was fixed at $3 \mathrm{~mL} / \mathrm{min}$. The observation height was $15 \mathrm{~mm}$ above the load coil. Further details on the instrumentation are 
available elsewhere (Roelandts and Michel, 1986; Roelandts, 1988a).

\section{Cation-Exchange Columns}

Borosilicate glass tubes of $10-\mathrm{mm}$ internal diameter filled with Dowex 50X8 cation-exchange resin (6-g dry weight) were used as columns. The settled resin height in the column was adjusted to $200 \mathrm{~mm}$. Before starting the separation, the columns were equilibrated by passing $50 \mathrm{~mL}$ of $1.75 M$ hydrochloric acid through them.

\section{Reagents}

\section{Cation-Exchange Resin}

The resin used was the Dowex 50X8 sulphonated cation exchanger (hydrogen form, 100 to 200 mesh) supplied by Fluka Co., Buchs, Switzerland. Before use, the fine particles were removed by several decantations.

\section{Rare Earth Elements Standard Solutions}

Individual REE solutions $(1,000 \mu \mathrm{g} / \mathrm{mL})$ were prepared by dissolving high purity ( 99.9 percent) REE oxides (Rare-Earth Products Ltd., London, U.K.) in hot nitric acid. Before weighing, the oxides were heated at $95^{\circ} \mathrm{C}$.

The single-element solutions were prepared by appropriate dilution of the stock solutions. All solutions were stored in polypropylene bottles having a total nitric acid content of 5 percent by volume.

\section{Chemicals}

All the acids used were analytical-reagent grade.

\section{Analytical Procedure \\ Dissolution of the Samples}

Samples 1-g each were digested on a water-bath using hydrofluoric-nitric acid attack and evaporated to dryness. The salts were dissolved in concentrated hydrochloric acid by heating. The solutions were again evaporated to dryness. The $\mathrm{HCl}$ digestion was repeated at least twice. Finally, the residues were dissolved in $1.75 \mathrm{M} \mathrm{HCl}$.

\section{Cation-Exchange Separation}

The $1.75 \mathrm{M} \mathrm{HCl}$ sample solution was passed through the preequilibrated resin column. After the elution of iron, calcium, and other unwanted matrix elements with $100 \mathrm{~mL}$ of $1.75 \mathrm{M} \mathrm{HCl}$, the REE adsorbed on the column were washed out with $75 \mathrm{~mL} 8 M \mathrm{HNO}_{3}$. All separations were done at an eluent flow rate of about $1 \mathrm{~mL} / \mathrm{min}$. Six separate exchange experiments were performed simultaneously.

The REE eluate was then evaporated to dryness, dissolved in 5-percent $(V / V)$ nitric acid, and diluted to 25
Table 1. Spectral interference correction coefficients

\begin{tabular}{|c|c|c|c|}
\hline \multirow{2}{*}{$\frac{\text { Element }}{\mathrm{La}}$} & \multirow{2}{*}{$\begin{array}{c}\begin{array}{c}\text { Wavelength } \\
\text { (in nanometers) }\end{array} \\
398.852\end{array}$} & \multicolumn{2}{|c|}{$\begin{array}{l}\text { Interfering rare earth elements } \\
\text { and correction coefficients }\end{array}$} \\
\hline & & & \\
\hline $\mathrm{Ce}$ & 418.660 & & \\
\hline $\mathrm{Nd}$ & 430.358 & & \\
\hline $\mathrm{Sm}$ & 359.260 & $\mathrm{Nd}(0.0372)$ & Gd (0.0313) \\
\hline $\mathrm{Eu}$ & 381.967 & $\mathrm{Nd}(0.0040)$ & \\
\hline $\mathrm{Gd}$ & 342.247 & $\mathrm{Ce}(0.0037)$ & \\
\hline Dy & 353.170 & $\mathrm{Nd}(0.0012)$ & \\
\hline $\mathrm{Yb}$ & 328.937 & & \\
\hline $\mathrm{Lu}$ & 261.542 & & \\
\hline
\end{tabular}

$\mathrm{mL}$. This solution was stored in a polypropylene bottle until the ICP-AES measurements were carried out.

\section{ICP-AES Measurements}

For each sample solution nebulized into the plasma, a stabilization time was set at 30-s ("preflush time") and followed by three 1-s signal integrations; the sample intensities were calculated as the arithmetic mean of these three readings. External calibration was made using singleelement solutions of REE (in 5-percent ( $V / V$ ) nitric acid). A 5-percent $(V / V)$ nitric acid solution was used to determine the true background under the peak. All intensities were corrected for background so obtained.

After the cation-exchange separation procedure, the only important spectral interferences that remained were those caused by overlap from other REE peaks. The REE analytical lines selected in this work are listed in table 1. The choice is a compromise between signal intensity and relative freedom from spectral interferences. Spectral profiles of these lines and correction coefficients for mutual REE interferences have been previously reported (Roelandts and Michel, 1986). They are largely dependent on the resolution of the spectrometer and may vary from instrument to instrument. A wavelength scan 0.1 nanometer $(\mathrm{nm})$ on each side of the Sm emission line at $359.260 \mathrm{~nm}$ $(10 \mu \mathrm{g} / \mathrm{mL} \mathrm{Sm} ; 100 \mu \mathrm{g} / \mathrm{mL}$ for other REE) is illustrated in figure 1. Overlap corrections introduced in this study are indicated in table 1 . For example, a sample that contains 1 $\mu \mathrm{g} / \mathrm{g} \mathrm{Nd}$ generates the equivalent of $0.0372 \mu \mathrm{g} / \mathrm{g} \mathrm{Sm}$; therefore, the contributions from $\mathrm{Nd}$ to $\mathrm{Sm}$ will be estimated by multiplying the $\mathrm{Nd}$ concentrations by 0.0372 and subtracting this product from the total measured Sm content.

\section{RESULTS AND DISCUSSION}

Three portions from the two bottles of SDO-1 were analyzed in random order for nine REE ( $\mathrm{La}, \mathrm{Ce}, \mathrm{Nd}, \mathrm{Sm}$, $\mathrm{Eu}, \mathrm{Gd}, \mathrm{Dy}, \mathrm{Yb}$, and $\mathrm{Lu}$ ). The experimental results of the individual samples (average of three measurements on the 
Table 2. Determination of nine rare earth elements in USGS Devonian Ohio Shale SDO-1

[All concentrations in $\mu \mathrm{g} / \mathrm{g}$ ]

\begin{tabular}{|c|c|c|c|c|c|c|c|}
\hline \multirow[b]{3}{*}{ Element } & \multicolumn{3}{|c|}{ Bottle 0189} & \multicolumn{3}{|c|}{ Bottle 0717} & \multirow[b]{3}{*}{ Mear } \\
\hline & \multicolumn{3}{|c|}{ Determination } & \multicolumn{3}{|c|}{ Determination } & \\
\hline & A & B & $\mathrm{C}$ & A & B & C & \\
\hline $\mathrm{La}$ & 36.2 & 35.6 & 35.6 & 36.0 & 35.9 & 36.0 & 35.9 \\
\hline $\mathrm{Ce}$ & 76.9 & 75.3 & 75.3 & 77.6 & 76.7 & 74.9 & 76.1 \\
\hline $\mathrm{Nd}$ & 37.9 & 38.0 & 38.7 & 38.5 & 38.1 & 38.3 & 38.3 \\
\hline $\mathrm{Sm}$ & 7.7 & 7.6 & 7.5 & 7.7 & 7.6 & 7.6 & 7.6 \\
\hline $\mathrm{Eu}$ & 1.64 & 1.66 & 1.65 & 1.65 & 1.67 & 1.69 & 1.66 \\
\hline $\mathrm{Gd}$ & 7.8 & 7.6 & 7.8 & 7.8 & 7.9 & 7.9 & 7.8 \\
\hline Dy & 6.1 & 6.0 & 6.0 & 6.1 & 6.1 & 6.1 & 6.1 \\
\hline $\mathrm{Yb}$ & 3.05 & 2.99 & 3.00 & 3.04 & 3.09 & 3.08 & 3.04 \\
\hline $\mathrm{Lu}$ & .45 & .44 & .44 & .45 & .45 & .47 & .45 \\
\hline
\end{tabular}

solution) are presented in table 2, including the mean of the six separate analyses. Based on these analyses, precision is estimated to be better than 1 percent.

The one-way analysis of variance was used with the ICP data to determine if the bottles are homogeneous with respect to REE content. Table 3 summarizes the calculations and the conclusions from the analysis of variance. The $F$ ratio calculated for REE in no case exceeds the allowable

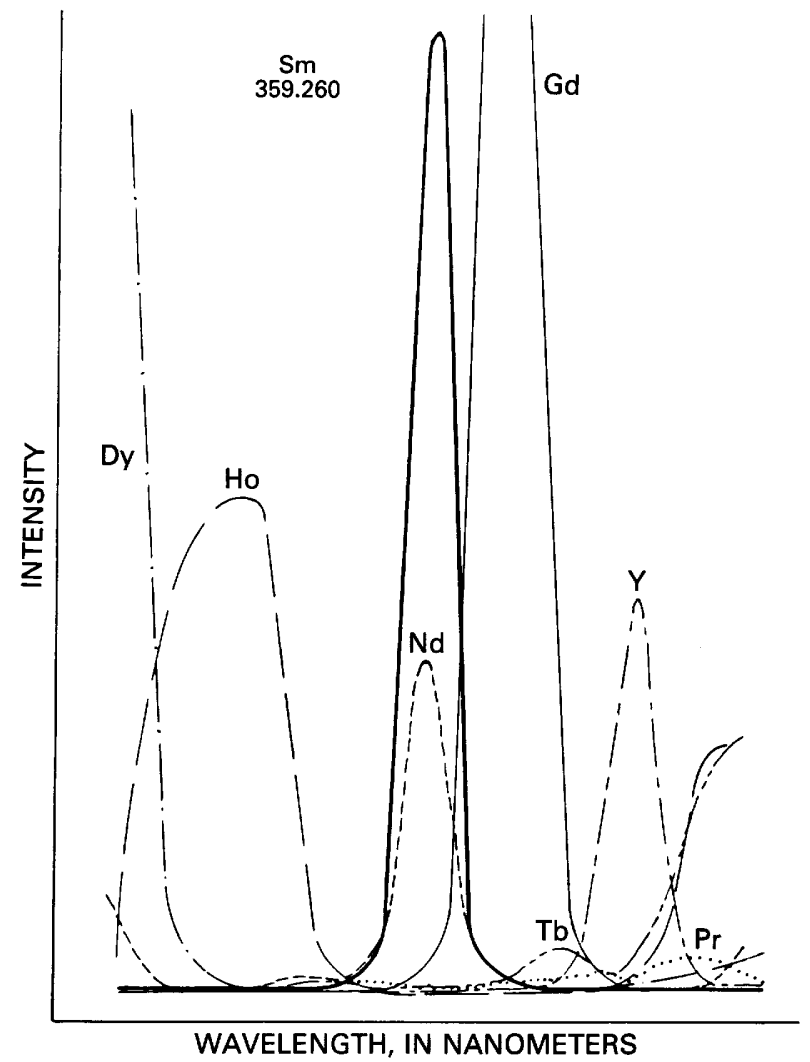

Figure 1. Wavelength scan of the $\mathrm{Sm} 359.260$ nanometer line $(10 \mu \mathrm{g} / \mathrm{mL} \mathrm{Sm} ; 100 \mu \mathrm{g} / \mathrm{mL}$ for other rare earth elements). value $F_{c}(0.95)(1,4)=7.7086$ as shown in the $F$ tables. Therefore, the statistical analysis demonstrated that SDO-1 may be considered homogeneous for the elements specified at the 95-percent confidence level. Because of the random selection of the bottles for the experiment, this conclusion may be extrapolated to the entire lot of bottles.

In table 4, the average REE concentrations obtained in the present work (bottle 0717) are compared with results for one analysis of this sample previously undertaken in our laboratory about 18 months ago. The agreement between these two sets of data is judged to be excellent and reflects the long-term reproducibility that can be achieved using the outlined method. Additionally, from this table it can be seen that our determinations compare favorably with the ICP-AES values reported by Crock and others (1986) and with the final recommended concentrations (Kane and others, 1990).

The accuracy of our ICP-AES method has been established by performing analyses on more that 40 different international geochemical reference materials (Roelandts and Michel, 1986; Roelandts, 1987, 1988a, 1988b, 1989 ) and through participation in a series of collaborative

Table 3. One-way analysis of variance [d.f., degree of freedom; NS, not significant]

\begin{tabular}{lcrrr}
\hline & \multicolumn{2}{c}{ Mean squares } & & \\
\cline { 2 - 3 } Element & d.f. $=1$ & d.f. $=4$ & F-ratio & Conclusion \\
\hline $\mathrm{La}$ & 0.04167 & 0.06167 & 0.6757 & $\mathrm{NS}$ \\
$\mathrm{Ce}$ & .48167 & 1.37167 & .3512 & $\mathrm{NS}$ \\
$\mathrm{Nd}$ & .01500 & .11500 & .1304 & $\mathrm{NS}$ \\
$\mathrm{Sm}$ & .00167 & .00667 & .2500 & $\mathrm{NS}$ \\
$\mathrm{Eu}$ & .00060 & .00025 & 2.4000 & $\mathrm{NS}$ \\
$\mathrm{Gd}$ & .01000 & .02000 & .5000 & $\mathrm{NS}$ \\
$\mathrm{Dy}$ & .00667 & .00167 & 4.0000 & $\mathrm{NS}$ \\
$\mathrm{Yb}$ & .00482 & .00087 & 5.5577 & $\mathrm{NS}$ \\
$\mathrm{Lu}$ & .00027 & .00008 & 3.2000 & $\mathrm{NS}$ \\
\hline
\end{tabular}


Table 4. Results obtained for USGS Devonian Ohio Shale SDO-1 in the present work compared with previous ICP data

[All concentrations in $\mu \mathrm{g} / \mathrm{g}$; ICP, inductively coupled plasma]

\begin{tabular}{|c|c|c|c|c|}
\hline Element & This work ${ }^{1}$ & Previous work ${ }^{1,2}$ & Crock and others, 1986 & Average $^{3}$ \\
\hline $\mathrm{La}$ & 36.0 & 35.9 & 35.6 & $* 38.5 \pm 4.4$ \\
\hline $\mathrm{Ce}$ & 76.4 & 76.2 & 71.8 & $* 79.3 \pm 7.8$ \\
\hline $\mathrm{Nd}$ & 38.3 & 38.2 & 36.3 & $36.6 \pm 3.3$ \\
\hline $\mathrm{Sm}$ & 7.6 & 7.6 & 7.6 & $7.7 \pm 0.81$ \\
\hline $\mathrm{Eu}$ & 1.67 & 1.68 & 1.65 & $1.6 \pm 0.22$ \\
\hline $\mathrm{Gd}$ & 7.8 & 7.8 & 7.8 & $7.4 \pm 1.9$ \\
\hline Dy & 6.1 & 5.9 & 6.1 & $* 6.0 \pm 0.65$ \\
\hline $\mathrm{Yb}$ & 3.07 & 3.04 & 3.11 & $* 3.4 \pm 0.46$ \\
\hline $\mathrm{Lu}$ & .46 & .45 & .44 & $.54 \pm 0.14$ \\
\hline
\end{tabular}

Table 5. Determination of nine rare earth elements in USGS sedimentary reference samples

[All concentrations in $\mu \mathrm{g} / \mathrm{g}$; ICP, inductively coupled plasma]

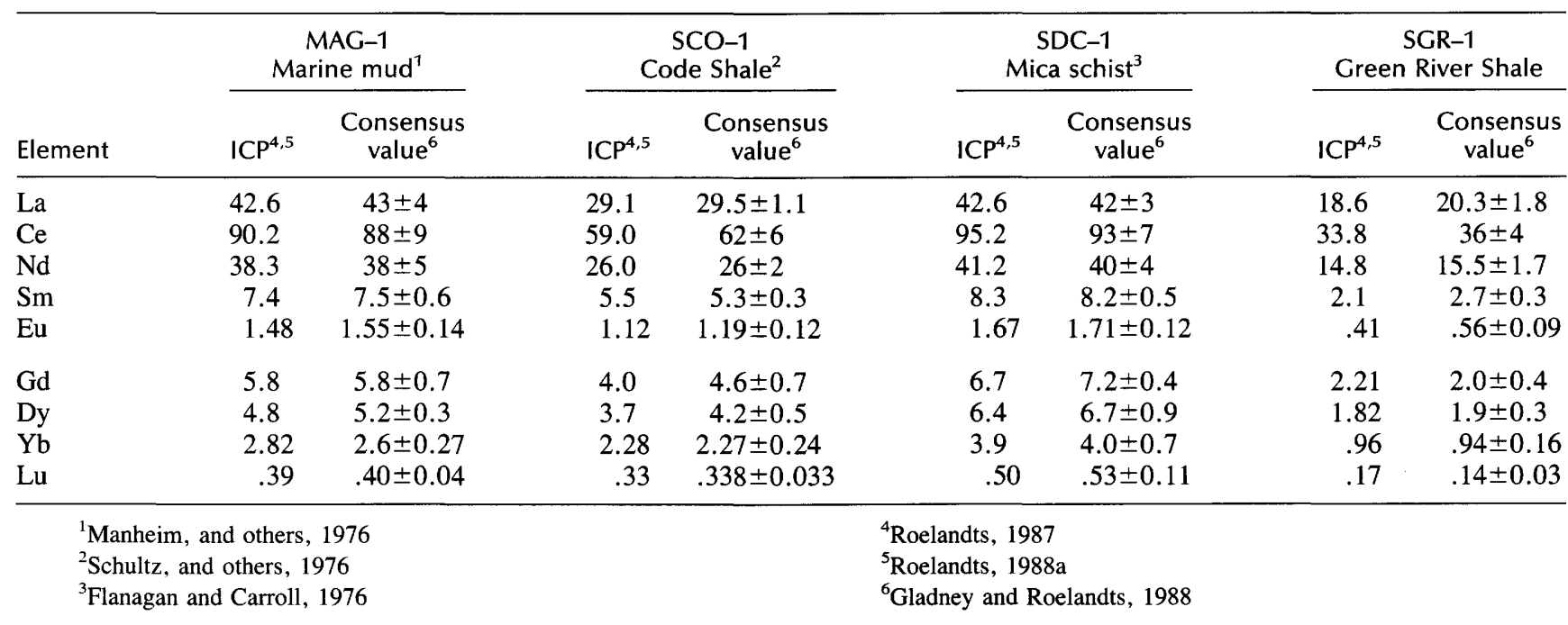

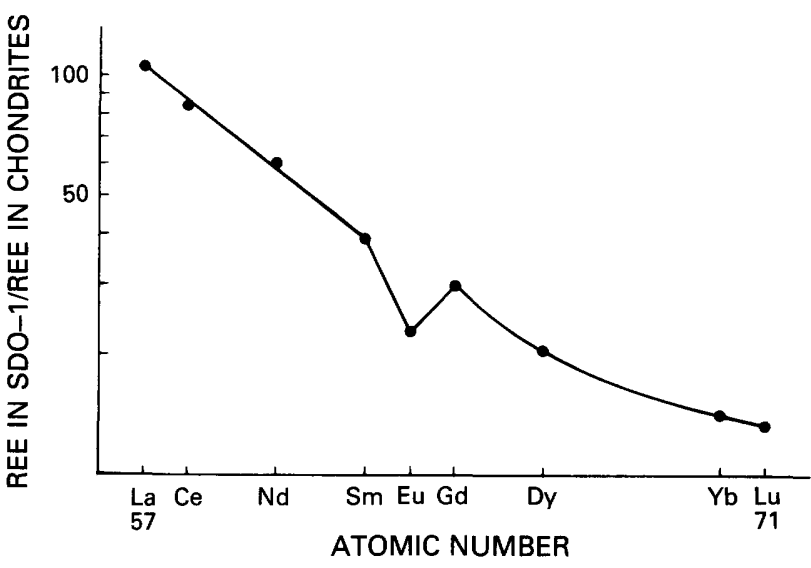

Figure 2. Chondrite-normalization pattern for the Devonian Ohio Shale SDO-1. studies. Good agreement between our ICP determinations and the preferred values for these reference materials is observed in most cases. Table 5 contains results of nine REE in USGS sedimentary reference samples. Comparison of our ICP values with the consensus values calculated by Gladney and Roelandts (1988) indicates an accuracy of \pm 4 percent. The absence of any serious systematic bias in our REE determinations is also evident and shows the validity of the analytical procedure.

The chondrite normalized concentrations for SDO-1 are plotted against the atomic number in figure 2 using the chondrite values reported by Laul and Rancitelli (1977). This procedure offers a quick evaluation of the quality of data for REE abundances. A smooth curve that is widely accepted as a quality criterion for REE analysis can be observed in our case. 


\section{ACKNOWLEDGMENTS}

We are grateful to V. Miocque and G. Delhaze for their assistance. The ICP equipment used for this study was purchased with funds from the Belgian "Fonds de la Recherche Fondamentale Collective," under contract no. 9.4569.83.

\section{REFERENCES}

Aulis, R., Bolton, Alexander, Doherty, William, Vander Voet, Anthony, and Wong, P., 1985, Determination of yttrium and selected rare-earth elements in geological materials using high performance liquid chromatography separation and ICP spectrometric detection: Spectromchimica Acta, v. 40B, p. 377-387.

Bolton, Alexander, Hwang, Jack, and Vander Voet, Anthony, 1983, The determination of scandium, yttrium and selected rare earth elements in geological materials by inductively coupled plasma optical emission spectrometry: Spectrochimica Acta, v. 38B, p. 165-174.

Brenner, I.B., Jones, E.A., Watson, A.E., and Steele, T.W., 1984, The application of a $\mathrm{N}_{2}$-Ar medium-power ICP and cation-exchange chromatography for the spectrometric determination of the rare-earth elements in geological materials: Chemical Geology, v. 45, p, 135-148.

Brenner, I.B., Watson, A.E., Steele, T.W., Jones, E.A., and Goncalves, M., 1981, Application of an argon-nitrogen inductively-coupled radio frequency plasma (ICP) to the analysis of geochemical and related materials for their rareearth contents: Spectrochimica Acta, v. 36B, p. 785-797.

Crock, J.G., and Lichte, F.E., 1982, Determination of rare-earth elements in geological materials by inductively coupled argon plasma/atomic emission spectrometry: Analytical Chemistry, v. 54 , p. $1329-1332$.

Crock, J.G., Lichte, F.E., Riddle, G.O., and Beech, C.L., 1986, Separation and preconcentration of the rare-earth elements and yttrium from geological materials by ion-exchange and sequential acid elution: Talanta, v. 33, p. 601-606.

Crock, J.G., Lichte, F.E., and Wildemann, T.R., 1984, The group separation of the rare-earth elements and yttrium from geologic materials by cation-exchange chromatography: Chemical Geology, v. 45, p. 149-163.

Flanagan, F.J., and Carroll, G.V., 1976, Mica-schist, SDC-1 from Rock Creek Park, Washington, D.C.: U.S. Geological Survey Professional Paper 840, p. 29-32.

Gladney, E.S., and Roelandts, Iwan, 1988, 1987 Compilation of elemental concentration data for USGS BHVO-1, MAG-1, QLO-1, RGM-1, SCo-1, SDC-1, SGR-1, and STM-1: Geostandards Newsletter, v. 12, p. 253-362.

Govindaraju, Kuppisami, and Mevelle, Guy, 1987, Fully automated dissolution and separation methods for inductively coupled plasma atomic emission spectrometry rock analysis. Application to the determination of rare-earth elements: Journal of Analytical Atomic Spectrometry, v. 2, p. $615-621$.
Jarvis, Ian, and Jarvis, K.E., 1985, Rare-earth element geochemistry of standard sediments - a study using inductively coupled plasma spectrometry: Chemical Geology, v. 53, p. 335-344.

1988, Determination of the rare-earth elements and yttrium in 37 international silicate reference materials by inductively coupled plasma-atomic spectrometry: Geostandards Newsletter, v.12, p. 1-12.

Kane, J.S., Arbogast, Belinda, and Leventhal, Joel, 1990, Characterization of Devonian Ohio Shale SDO-1 as a USGS geochemical reference sample: Geostandards Newsletter, v. 14, p. 169-196.

Laul, J.C., and Rancitelli, L.A., 1977, Multielement analysis by sequential instrumental and radiochemical neutron activation: Journal of Radioanalytical Chemistry, v. 38, p. 461-475.

Manheim, F.T., Hathaway, J.C., Flanagan, F.J., and Fletcher, J.D., 1976, Marine Mud, MAG-1, from the Gulf of Maine: U.S. Geological Survey Professional Paper 840, p. 25-28.

Roelandts, Iwan, 1987, Determination of nine rare-earth elements in eight USGS reference samples and in the ANRT glauconite, GL-O, by sequential inductively coupled argon plasma spectrometry: Geostandards Newsletter, v. 11, p. 49-74.

1988a, Comparison of inductively coupled plasma and neutron activation analysis for precise and accurate determination of nine rare-earth elements in geological materials: Chemical Geology 67, p. 171-180.

1988b, Application of inductively coupled plasma spectrometry to the determination of nine rare earth elements in nine new U.S. Geological Survey reference samples: Atomic Spectroscopy, v. 9, p. 49-54.

1989, Inductively coupled plasma determination of nine rare-earth elements in nine Geological Survey of Japan rock reference samples: ICP Information Newsletter, v. 15, p. 309-310.

-1990, Inductively coupled plasma determination of nine rare-earth elements in sixty international geochemical reference samples: Geostandards Newsletter, v. 14, p. 137-147.

Roelandts, Iwan, and Michel, G., 1986, Sequential inductively coupled plasma determination of some rare-earth elements in five French geostandards: Geostandards Newsletter, v. 10, p. 135-154.

Schultz, L.G., Tourtelot, H.A., and Flanagan, F.J., 1976, Cody Shale SCo-1, from Natrona County, Wyoming: U.S. Geological Survey Professional Paper 840, p. 21-23.

Sulcek, Zdenek, Rubeska, Ivan, Sixta, Vaclav, and Paukert, Tomas, 1989, Determination of rare-earth elements and yttrium in rocks using the Plasma II ICP emission spectrometer: Atomic Spectroscopy, v. 10, p. 4-9.

Walsh, J.N., Buckley, F., and Barker, J., 1981, The simultaneous determination of rare-earth elements in rocks using inductively coupled plasma source spectrometry: Chemical Geology, v. 33, p. 141-153.

Watkins, P.J., and Nolan, J., 1990, Determination of rare-earth elements, scadium, yttrium and hafnium in 32 geochemical reference materials using inductively coupled plasma atomic emission spectrometry: Geostandards Newsletter, v. 14, p. 11-20. 
Yoshida, K., Fuwa, K., and Haraguchi, H., 1983, Determination of 15 rare-earth elements by liquid chromatography with inductively coupled plasma atomic emission spectrometric detection: Chemical Letter, p. 1879-1882.
Zachmann, D.W., 1988, Matrix effects in the separation of rare earth elements, scandium, and yttrium and their determination by inductively coupled plasma emission spectrometry: Analytical Chemistry, v. 60 , p. $420-427$. 
Chapter D

\section{Instrumental Neutron Activation Analysis of Devonian Ohio Shale SDO-1}

By GREGORY A. WANDLESS

U.S. GEOLOGICAL SURVEY BULLETIN 2046

THE USGS REFERENCE SAMPLE DEVONIAN OHIO SHALE SDO-1 



\title{
CONTENTS
}

\author{
Abstract D1 \\ Introduction D1 \\ Experiment Parameters D1 \\ Results D1 \\ Discussion D2 \\ Acknowledgments D4 \\ References D4
}

FIGURES

1. The $\mathrm{Lu} / \mathrm{U}(\mathrm{Np})$ doublet prior to interactive fitting $\mathbf{D 4}$

2. The $\mathrm{Lu} / \mathrm{U}(\mathrm{Np})$ doublet after interactive fitting $\mathbf{D} 4$

3. Graph showing chondrite normalized rare-earth plot of SDO-1 D4

\section{TABLES}

1. Instrumental neutron activation analysis results for USGS geochemical reference sample SDO-1 D2

2. Analysis of variance results for INAA of SDO-1 D3

3. Fission product correction factors D3 



\title{
Instrumental Neutron Activation Analysis of Devonian Ohio Shale SDO-1
}

\author{
By Gregory A. Wandless
}

\begin{abstract}
The U.S. Geological Survey geochemical reference sample Devonian Ohio Shale SDO-1 was analyzed by instrumental neutron activation analysis. The relatively high uranium concentration in SDO-1 caused several isotopic and spectral interferences that had to be resolved. Special data processing techniques were applied to the assay of lutetium to remove spectral interferences from uranium, and significant corrections had to be applied to several elements for fission product interferences. A correction for the ruthenium fission product interference has been made to barium since the publication of the original SDO-1 compilation, resulting in a significant improvement in accuracy. Four replicates from two randomly selected bottles were analyzed; and the precision was estimated to be 5 percent or better for a majority of elements, and the accuracy was demonstrated by no rejection of data in the determination of "recommended values." Analysis of variance shows no significant variation between bottles.
\end{abstract}

\section{INTRODUCTION}

A discussion of the purpose for collecting SDO-1, sample description and preparation, and the analytical program have already been presented (Kane and others, 1990). Applying instrumental neutron activation analysis (INAA) to the analysis of SDO-1 provides an efficient means for characterization because the method can provide precise data for approximately 30 major, minor, and trace elements in a single sample aliquot without chemical treatment. The underlying principles and the application of INAA to geologic samples have been described numerous times (Gordon and others, 1968; Hertogen and Gijbels, 1971; Baedecker and others, 1977; Jacobs and others, 1977; Laul, 1979; Baedecker and McKown, 1987). The most significant problems associated with the analysis of SDO-1 will be discussed in detail.

Manuscript approved for publication September 15, 1992.

\section{EXPERIMENT PARAMETERS}

The samples of SDO-1 as received for analysis needed no further treatment for activation analysis. Four aliquots of SDO-1 from each of two bottles, two multielement standards, a standard for calcium and titanium, and the U.S. Geological Survey (USGS) geochemical reference sample SGR-1, all weighing approximately $0.5 \mathrm{~g}$, were weighed into $2 / 5$-dram polyethylene vials. The vials were heat sealed, placed in a polyethylene bag, and packed into an aluminum can for irradiation.

Samples and standards were irradiated simultaneously for 8 hours in the A-2 position of the Texas A\&M reactor facility, providing a neutron flux of $2 \times 10^{12}$ neutrons $\mathrm{cm}^{-2} \mathrm{~s}^{-1}$. After irradiation, the samples were returned after decaying for 7 days. The vials were then unpacked, washed externally with water and alcohol, and placed in clean 2-dram polyethylene vials for counting.

Samples and standards were counted on coaxial, high-purity germanium (HPGe) and low-energy photon detectors (LEPD) 7 days after irradiation for 1 hour each, after 14 days on a HPGe for 1 hour, and again on the HPGe and LEPD after 2 months for 2 hours. Gamma-ray spectra were acquired on a Nuclear Data 6700 multichannel analyzer and transferred to a VAX 11/780 for data reduction. Spectra were analyzed using the SPECTRA program (Baedecker and Grossman, 1989).

\section{RESULTS}

Results for the eight replicate analyses of SDO-1 are given in table 1. Means and standard deviations were computed assuming no between-bottle variance. The precision for the majority of elements is 5 percent or better, and only two ( $\mathrm{Ti}$ and $\mathrm{Zr}$ ) have a coefficient of variation of greater than 10 percent. With regard to accuracy, it is noted that only data for barium were rejected during the initial determination of "recommended values" or averages for the SDO-1 standard. Since the initial analysis, corrections for fission-product interference on barium have been determined and have improved the accuracy of the barium data such that those results can now be included in the derivation of recommended values. To evaluate sample homogeneity 
Table 1. Instrumental neutron activation analysis results for USGS geochemical reference sample SDO-1

\begin{tabular}{|c|c|c|c|c|c|c|c|c|c|c|}
\hline & \multicolumn{4}{|c|}{ Bottle 699} & \multicolumn{4}{|c|}{ Bottle 761} & \multirow[b]{2}{*}{ Mean } & \multirow{2}{*}{$\begin{array}{l}\text { Standard } \\
\text { deviation }\end{array}$} \\
\hline & Replicate 1 & Replicate 2 & Replicate 3 & Replicate 4 & Replicate 1 & Replicate 2 & Replicate 3 & Replicate 4 & & \\
\hline $\begin{array}{l}\mathrm{Na} \text { (wt. \%) } \\
\mathrm{K} \\
\mathrm{Ca} \\
\mathrm{Ti} \\
\mathrm{Fe}\end{array}$ & $\begin{aligned} & 0.266 \\
& 2.76 \\
< & 12 \\
< & <.7 \\
& 6.56\end{aligned}$ & $\begin{array}{c}0.269 \\
2.71 \\
<12 \\
<.6 \\
\quad 6.59\end{array}$ & $\begin{array}{r}0.269 \\
2.65 \\
<11 \\
.82 \\
6.53\end{array}$ & $\begin{array}{c}0.269 \\
2.71 \\
<11 \\
<.9 \\
6.56\end{array}$ & $\begin{array}{r}0.264 \\
2.67 \\
<14 \\
.53 \\
6.40\end{array}$ & $\begin{array}{c}0.272 \\
2.82 \\
<14 \\
<.6 \\
\quad 6.50\end{array}$ & $\begin{array}{r}0.269 \\
2.66 \\
<11 \\
.62 \\
6.58\end{array}$ & $\begin{array}{c}0.269 \\
2.68 \\
<11 \\
<.6 \\
\quad 6.61\end{array}$ & $\begin{array}{l}0.268 \\
2.71 \\
- \\
.66 \\
6.54\end{array}$ & $\begin{array}{c}0.0024 \\
.050 \\
- \\
.150 \\
.067\end{array}$ \\
\hline $\begin{array}{l}\mathrm{Sc}(\mu \mathrm{g} / \mathrm{g}) \\
\mathrm{Cr} \\
\mathrm{Co} \\
\mathrm{Ni} \\
\mathrm{Zn}\end{array}$ & $\begin{array}{l}12.4 \\
62.7 \\
45.0 \\
95 \\
62\end{array}$ & $\begin{array}{l}12.6 \\
64 \\
45.3 \\
101 \\
65\end{array}$ & $\begin{array}{l}12.3 \\
63 \\
44.2 \\
83 \\
61\end{array}$ & $\begin{array}{l}12.4 \\
64.5 \\
44.6 \\
92 \\
61\end{array}$ & $\begin{array}{l}12.1 \\
62.5 \\
43.7 \\
86 \\
60\end{array}$ & $\begin{array}{l}12.3 \\
64.3 \\
44.3 \\
86 \\
58\end{array}$ & $\begin{array}{c}12.4 \\
64.6 \\
45.1 \\
100 \\
58\end{array}$ & $\begin{array}{l}12.4 \\
63.5 \\
44.9 \\
91 \\
63\end{array}$ & $\begin{array}{l}12.4 \\
63.6 \\
44.6 \\
92 \\
61\end{array}$ & $\begin{array}{l}.140 \\
.83 \\
.54 \\
6.6 \\
2.36\end{array}$ \\
\hline $\begin{array}{l}\mathrm{As} \\
\mathrm{Se} \\
\mathrm{Rb} \\
\mathrm{Sr} \\
\mathrm{Zr}\end{array}$ & $\begin{array}{r}63.0 \\
7.3 \\
127 \\
<230 \\
124\end{array}$ & $\begin{array}{r}63.2 \\
6.3 \\
133 \\
138 \\
<260\end{array}$ & $\begin{array}{l}61.9 \\
<5 \\
129 \\
140 \\
100\end{array}$ & $\begin{array}{r}63.4 \\
6.6 \\
129 \\
140 \\
140\end{array}$ & $\begin{array}{l}62.7 \\
<5 \\
125 \\
158 \\
102\end{array}$ & $\begin{array}{c}62.7 \\
<8 \\
130 \\
140 \\
84\end{array}$ & $\begin{array}{l}62.6 \\
<8 \\
125 \\
130 \\
160\end{array}$ & $\begin{array}{c}62.4 \\
<5 \\
126 \\
140 \\
<130\end{array}$ & $\begin{array}{c}62.7 \\
6.7 \\
128 \\
141 \\
118\end{array}$ & $\begin{array}{c}.47 \\
.51 \\
2.80 \\
8.4 \\
28.4\end{array}$ \\
\hline $\begin{array}{l}\mathrm{Mo} \\
\mathrm{Sb} \\
\mathrm{Cs} \\
\mathrm{Ba} \\
\mathrm{La}\end{array}$ & $\begin{array}{c}123 \\
4.66 \\
6.41 \\
404 \\
33.1\end{array}$ & $\begin{array}{c}126 \\
4.66 \\
6.46 \\
383 \\
33.6\end{array}$ & $\begin{array}{c}127 \\
4.62 \\
6.39 \\
409 \\
33.2\end{array}$ & $\begin{array}{c}129 \\
4.52 \\
6.56 \\
397 \\
33.6\end{array}$ & $\begin{array}{c}125 \\
4.37 \\
6.29 \\
394 \\
33.0\end{array}$ & $\begin{array}{c}126 \\
4.48 \\
6.44 \\
413 \\
33.1\end{array}$ & $\begin{array}{c}124 \\
4.68 \\
6.42 \\
423 \\
33.1\end{array}$ & $\begin{array}{c}127 \\
4.68 \\
6.38 \\
406 \\
33.4\end{array}$ & $\begin{array}{c}126 \\
4.58 \\
6.42 \\
403 \\
33.3\end{array}$ & $\begin{array}{c}1.90 \\
.115 \\
.070 \\
12.3 \\
.240\end{array}$ \\
\hline $\begin{array}{l}\mathrm{Ce} \\
\mathrm{Nd} \\
\mathrm{Sm} \\
\mathrm{Eu} \\
\mathrm{Tb}\end{array}$ & $\begin{array}{l}69.7 \\
31 \\
7.56 \\
1.46 \\
1.13\end{array}$ & $\begin{array}{l}72.2 \\
33 \\
7.73 \\
1.51 \\
1.17\end{array}$ & $\begin{array}{l}69.5 \\
31 \\
7.68 \\
1.44 \\
1.20\end{array}$ & $\begin{array}{l}70.9 \\
33 \\
7.78 \\
1.48 \\
1.17\end{array}$ & $\begin{array}{l}68.6 \\
32 \\
7.60 \\
1.44 \\
1.13\end{array}$ & $\begin{array}{l}70.4 \\
33 \\
7.60 \\
1.47 \\
1.10\end{array}$ & $\begin{array}{l}70.8 \\
33 \\
7.60 \\
1.47 \\
1.18\end{array}$ & $\begin{array}{l}70.6 \\
33 \\
7.69 \\
1.46 \\
1.13\end{array}$ & $\begin{array}{l}70.3 \\
32 \\
7.66 \\
1.47 \\
1.15\end{array}$ & $\begin{array}{l}1.08 \\
.80 \\
.070 \\
.0220 \\
.030\end{array}$ \\
\hline $\begin{array}{l}\text { Yb } \\
\text { Lu } \\
\text { Hf } \\
\text { Ta } \\
\text { Th }\end{array}$ & $\begin{array}{r}3.43 \\
.48 \\
4.43 \\
1.10 \\
9.65\end{array}$ & $\begin{array}{r}3.42 \\
.49 \\
4.46 \\
1.08 \\
9.91\end{array}$ & $\begin{array}{r}3.38 \\
.48 \\
4.38 \\
1.07 \\
9.70\end{array}$ & $\begin{array}{r}3.47 \\
.50 \\
4.37 \\
1.09 \\
9.85\end{array}$ & $\begin{array}{r}3.27 \\
.47 \\
4.27 \\
1.06 \\
9.60\end{array}$ & $\begin{array}{r}3.25 \\
.47 \\
4.39 \\
.99 \\
9.71\end{array}$ & $\begin{array}{r}3.49 \\
.47 \\
4.38 \\
1.07 \\
9.86\end{array}$ & $\begin{array}{r}3.40 \\
.48 \\
4.37 \\
1.09 \\
9.85\end{array}$ & $\begin{array}{r}3.39 \\
.48 \\
4.38 \\
1.07 \\
9.77\end{array}$ & $\begin{array}{l}.080 \\
.0110 \\
.050 \\
.030 \\
.115\end{array}$ \\
\hline U & 40.4 & 41.2 & 41.3 & 40.8 & 40.4 & 40.4 & 40.5 & 41.0 & 40.8 & .38 \\
\hline
\end{tabular}

for the elements determined by INAA, an analysis of variance was performed on the data, and the $\mathrm{F}$-statistic was tabulated in table 2. Since the critical F-value for the given degrees of freedom was not exceeded in any case, the hypothesis that there is no significant variation between bottles is accepted.

\section{DISCUSSION}

Baedecker and McKown (1987) give a detailed description of the factors that can affect the precision and accuracy of INAA; and for the majority of the trace elements determined in SDO-1, those factors were accounted for or insignificant during the analysis. For example, errors due to poor counting statistics were unimportant because of the relatively high count rates obtained during the analysis of SDO-1. However, the relatively high concentration of uranium $(40 \mu \mathrm{g} / \mathrm{g})$ introduced two forms of interferences that required evaluation for their effects on the analysis of SDO-1.
During neutron irradiation, ${ }^{235} \mathrm{U}$ and ${ }^{238} \mathrm{U}$ undergo fission, producing a host of daughter products called fission products. Some of these fission products are identical to the indicator radionuclides produced by the neutron irradiation of certain trace elements in SDO-1 (isotopic interference). Others have gamma-ray lines close to the lines of interest (spectral interferences). Failing to correct for these interferences will produce anomalous results for some elements. There are seven trace elements determined by INAA that can have significant interferences from uranium-fission products: $\mathrm{Ba}, \mathrm{Mo}, \mathrm{Zr}, \mathrm{La}, \mathrm{Ce}, \mathrm{Nd}$, and $\mathrm{Sm}$. Corrections for the isotopic interference from fission products can be determined by theoretical calculations or by irradiating a known quantity of uranium and measuring the quantity of those elements produced. The analytical data can then be corrected based on a determination of the concentration of uranium in the sample using the $277 \mathrm{keV}$ gamma-ray from ${ }^{239} \mathrm{~Np}$. Table 3 gives the correction factors used in this study that are consistent with the recent review of the available literature of fission-product corrections used in activation analysis (Landsberger, 1989). 
Table 2. Analysis of variance results for INAA of SDO-1. [F-critical $(95 \%)$ for $\mathrm{Sr} 6.6079, \mathrm{Zr} 7.7087$, all others 5.9874 ; insufficient data]

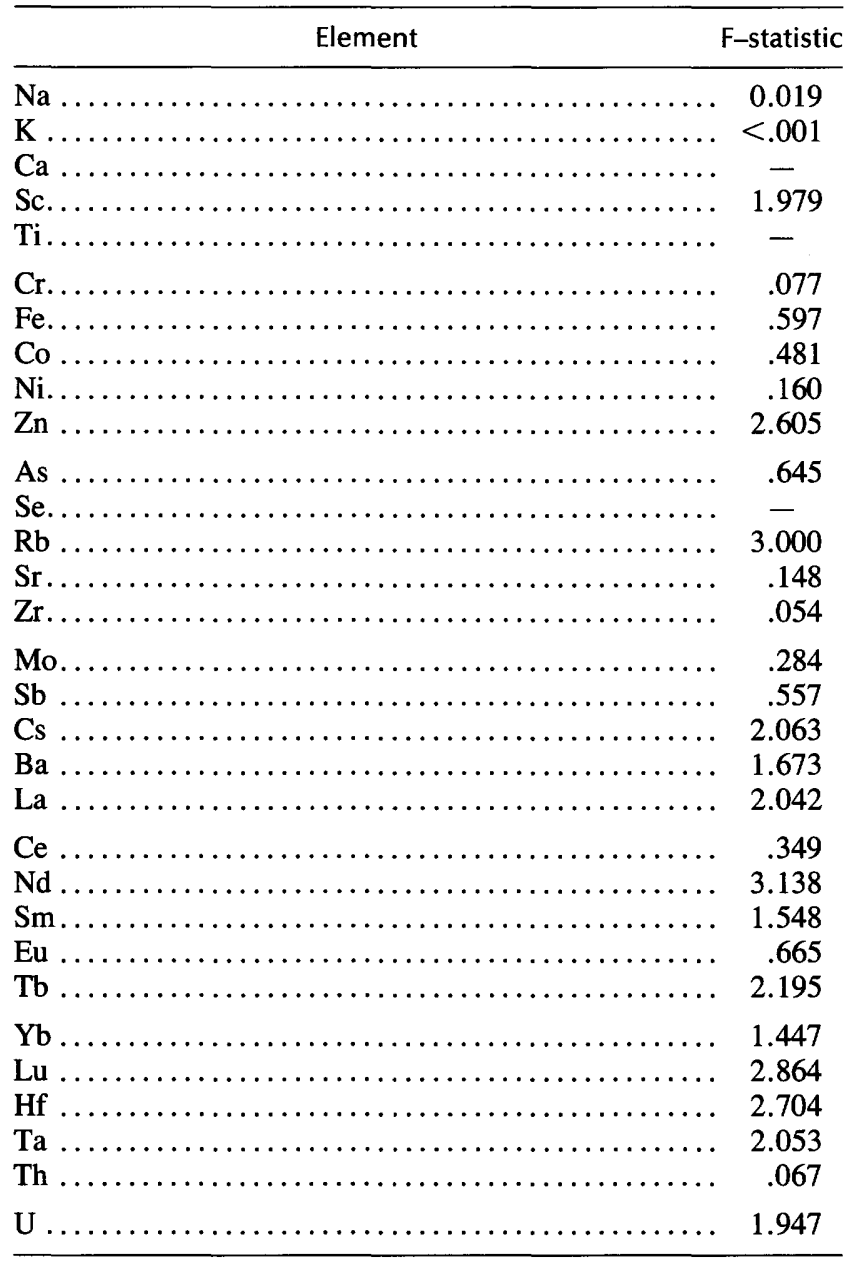

The determination of barium is complicated by the presence of fission product ${ }^{103} \mathrm{Ru}$. The $497.1 \mathrm{keV}$ line from ${ }^{103} \mathrm{Ru}$ interferes with the principle gamma-ray of ${ }^{131} \mathrm{Ba}$ at $496.4 \mathrm{keV}$. The half-life of ${ }^{131} \mathrm{Ba}$ is 12 days and of ${ }^{103} \mathrm{Ru}$ is 39.4 days; therefore, the longer the delay in counting for ${ }^{131} \mathrm{Ba}$, the more significant is the interference from ${ }^{103} \mathrm{Ru}$. Since no suitable interference-free line of ${ }^{103} \mathrm{Ru}$ is available to compute a correction factor for spectral interference, the factor was determined by irradiating an aliquot of uranium standard and determining the amount of ${ }^{103} \mathrm{Ru}$ produced by uranium fission. This time-dependent correction can then be applied after determination of the uranium abundance. In the initial compilation of SDO-1 analysis (Kane and others, 1990), a correction for this interference had not been applied, and the results from the $124 \mathrm{keV}$ line in the first planar count were unacceptable. In this work, the correction has been applied to the barium results determined using the $496 \mathrm{keV}$ line, and the agreement of the corrected results with "recommended values" is significantly improved. In an earlier compilation of SDO-1 (Kepferle and others,
Table 3. Fission product correction factors (apparent $\mu \mathrm{g} / \mathrm{g}$ element/ $\mu \mathrm{g} / \mathrm{g} \mathrm{U}$ )

\begin{tabular}{|c|c|}
\hline \multicolumn{2}{|c|}{${ }^{95} \mathrm{Zr} \ldots \ldots \ldots \ldots \ldots \ldots 11.3$} \\
\hline${ }^{99} \mathrm{Mo}$. & .85 \\
\hline${ }^{141} \mathrm{Ce}$. & .29 \\
\hline${ }^{147} \mathrm{Nd}$. & .23 \\
\hline${ }^{153} \mathrm{Sm}$ & .00008 \\
\hline${ }^{140} \mathrm{La}$. & $.002693\left[\exp \left(0.01497 t_{d}\right)-1.0\right]$ \\
\hline${ }^{131} \mathrm{Ba}$. & $2.8\left[\exp \left(0.0402 t_{d}\right)\right]$ \\
\hline
\end{tabular}

1985), the barium data also suffer from the same fissionproduct interference.

Unlike the spectral interference from a different nuclide produced by uranium fission, the interferences to $\mathrm{Mo}, \mathrm{Zr}, \mathrm{La}, \mathrm{Ce}, \mathrm{Nd}$, and $\mathrm{Sm}$ are caused by the formation of the indicator radionuclides of those elements by uranium fission. The correction factors for these element were determined both experimentally and theoretically by calculation (Baedecker and McKown, 1987). The interference correction to $\mathrm{La}$ is complicated because the fission of uranium produces the $12.8 \mathrm{~d}{ }^{140} \mathrm{Ba}$ isotope that then decays to the $40.3 \mathrm{~h}{ }^{140} \mathrm{La}$. Therefore, the amount of interference is dependent upon the growth and decay of ${ }^{140} \mathrm{La}$ during the delay time, $t_{d}$, between irradiation and counting. The corrections to the $\mathrm{La}$ abundances were made using the time-dependent equation in table 3 . The constants were determined using the Bateman equation.

All these corrections are applicable to the neutron activation analysis of a material containing uranium, and even in geochemical samples containing "normal" relative concentrations of uranium, the interference from fission products can be significant. For example, the SGR-1 sample included in this study required a 16-percent correction to Mo values and 2.6-percent and 4-percent corrections to the $\mathrm{La}$ and $\mathrm{Ce}$ data, respectively. The high relative concentration of $U$ in SDO-1 produced corrections of 30 percent for Mo and $\mathrm{Nd}$ and 11 percent and 16 percent for $\mathrm{La}$ and $\mathrm{Ce}$, respectively; and the apparent concentration of $\mathrm{Zr}$ due to fission products was $600 \mu \mathrm{g} / \mathrm{g}$.

Spectral interferences from the high uranium content also complicated the determination of $\mathrm{Lu}$. The $209.8 \mathrm{keV}$ gamma-ray line of ${ }^{239} \mathrm{~Np}$, an activation product of ${ }^{238} \mathrm{U}$, is $1.4 \mathrm{keV}$ away from the principal gamma-ray line of ${ }^{177} \mathrm{Lu}$ used to assay Lu. The resolution of the HPGe detectors used was approximately $1.2 \mathrm{keV}$ in this region $(1.85 \mathrm{keV}$ FWHM at the $1332 \mathrm{keV}$ line of $\mathrm{Co}$ ); and because complete resolution of the uranium peak from the assay peak for $\mathrm{Lu}$ is not possible, a doublet results. If the $\mathrm{U} / \mathrm{Lu}$ ratio is small, the interference on Lu by $U$ is small and can be determined using other uranium lines and the known intensity ratios. However, as the $\mathrm{U} / \mathrm{Lu}$ ratio increases, the size of the uranium peak increases, and the determination of $\mathrm{Lu}$ becomes increasingly difficult. The doublet produced by $U$ $\left({ }^{239} \mathrm{~Np}\right)$ and $\mathrm{Lu}$ for SDO-1 is shown in figure 1 . The relative 


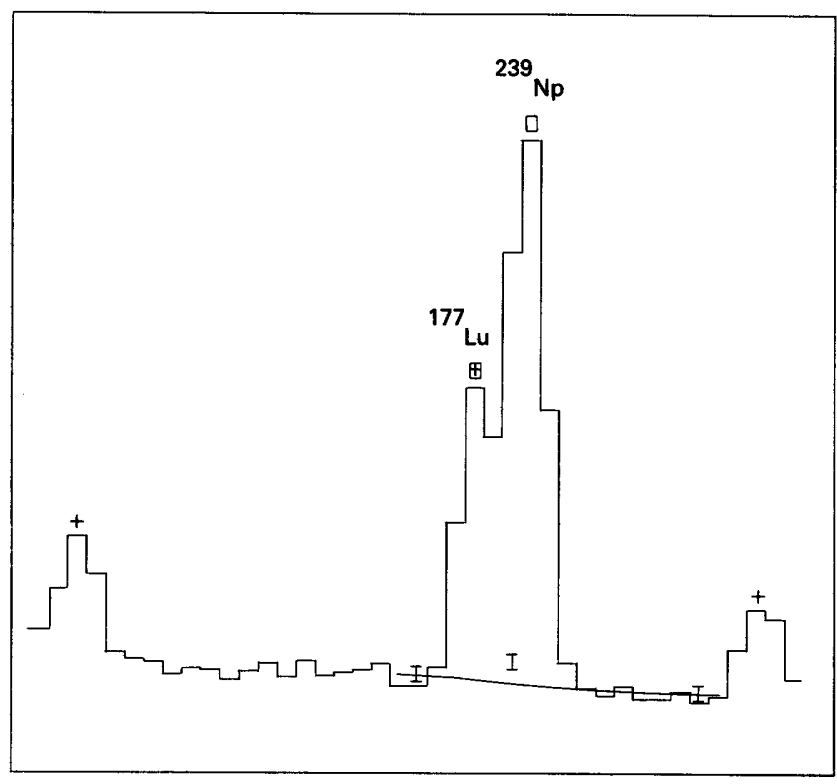

Figure 1. The $L u / U(N p)$ doublet prior to interactive fitting.

concentrations of $\mathrm{U}$ and $\mathrm{Lu}$ in SDO-1 still permitted the determination of $\mathrm{Lu}$ by performing interactive graphic plotting and fitting of the pair as described by Grossman and Baedecker (1986). Figure 2 shows the results of fitting and the residuals of the fit. Agreement between the 7- and 14-day counts suggests that the fitting routine successfully determined the peak areas for $\mathrm{Lu}$ and $\mathrm{U}$. The accuracy of the Lu data is often evaluated by looking at the relative positions of $\mathrm{Yb}$ and $\mathrm{Lu}$ in a chondrite normalized plot of

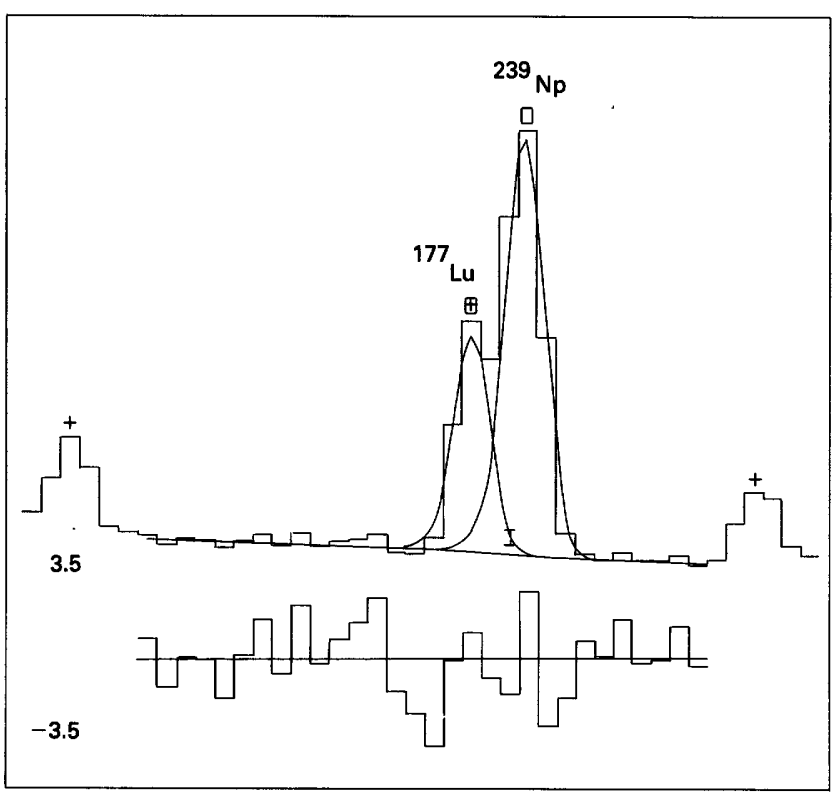

Figure 2. The $L u / U(N p)$ doublet after interactive fitting.

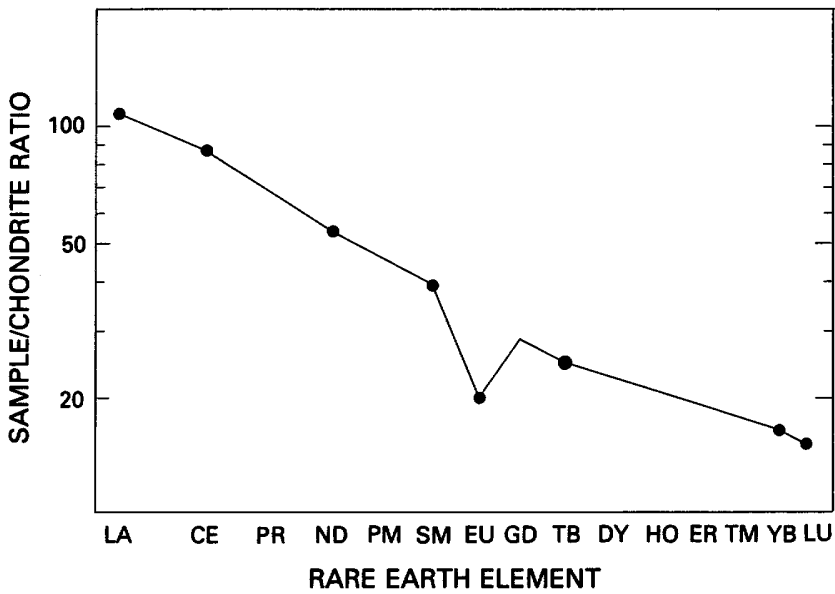

Figure 3. Chondrite normalized rare earth plot of SDO-1.

rare earth abundances. Figure 3 shows such a plot, and the smooth transition from $\mathrm{Yb}$ to $\mathrm{Lu}$ indicates high-quality $\mathrm{Lu}$ data.

\section{ACKNOWLEDGMENTS}

My thanks to Mike Pickering for his dedication during the weighing and data-acquisition phases of this project and to Phil Baedecker and Jeff Grossman for their technical and editorial suggestions.

\section{REFERENCES}

Baedecker, P.A., and Grossman, J.N., 1989, The computer analysis of high resolution gamma-ray spectra from instrumental activation analysis experiments: U.S. Geological Survey Open-File Report 89-454.

Baedecker, P.A., and McKown, D.M., 1987, Instrumental neutron activation analysis of geochemical samples, in Baedecker, P.A., ed., Methods for Geochemical Analysis: U.S. Geological Survey Bulletin 1770, p. H1-H14.

Baedecker, P.A., Rowe, J.J., and Steinnes, Eiliv, 1977, Application of epithermal neutron activation in multielement analysis of silicate rocks employing both coaxial $\mathrm{Ge}(\mathrm{Li})$ and low energy photon detector systems: Journal of Radioanalytical and Nuclear Chemistry, v. 39, p. 239-254.

Gordon, G.E., Randle, Keith, Goles, G.G., Corliss, J.B., Beeson, M.H., and Oxley, S.S., 1968, Instrumental activation analysis of standard rocks with high-resolution X-ray detectors: Geochemica et Cosmochimica Acta, v. 32, p. 369-396.

Grossman, J.N., and Baedecker, P.A., 1986, Computer graphics for quality control in the INAA of geological samples: Journal of Radioanalytical and Nuclear Chemistry, v. 113, p. 43-59.

Hertogen, Jan, and Gijbels, R., 1971, Instrumental neutron activation analysis of rocks with low-energy photon detector: Analytica Chimica Acta, v. 56, p. 61-82. 
Jacobs, J.W., Korotev, R.L., Blanchard, D.P., and Haskin, L.A., 1977, A well tested procedure for instrumental neutron activation analysis of silicate rocks and minerals: Journal of Radioanalytical and Nuclear Chemistry, v. 40, p. 93-114.

Kane, J.S., Arbogast, Belinda, and Leventhal, Joel, 1990, Characterization of Devonian Ohio Shale SDO-1 as a USGS geochemical reference sample: Geostandards Newsletter, v. 14, p. 169-196.
Kepferle, R.C., de Witt, Wallace, Jr., and Flanagan, F.J., 1985, Ohio Shale (Devonian), SDO-1, from Rowan County, Kentucky: U.S. Geological Survey Open-File Report, p. 85-145. Landsberger, Sheldon, 1989, Update of uranium fission interferences in neutron activation analysis: Chemical Geology, v. 77, 65-70.

Laul, J.C., 1979, Neutron activation analysis of geological materials, Atomic Energy Review, v. 17, p. 603-695. 

Chapter E

Relation Between Volatile Components and Loss on Ignition as Applied to the Analysis of USGS Reference Devonian Ohio Shale SDO-1 and Rare Earth Element Analyses

By MIROSLAV HUKA and IVAN RUBESKA

U.S. GEOLOGICAL SURVEY BULLETIN 2046

THE USGS REFERENCE SAMPLE DEVONIAN OHIO SHALE SDO-1 



\title{
CONTENTS
}

\author{
Abstract E1 \\ Introduction E1 \\ Relation of Volatile Components to LOI E1 \\ Normal Igneous Rocks E1 \\ The Effect of Sulfidic Sulfur E2 \\ The Effect of Inherent Sulfate Occurrences on LOI E3 \\ The Effect of Organic Carbon on LOI E3 \\ Experiment Parameters E3 \\ Results and Discussion E4 \\ Conclusion E4 \\ References E5
}

\section{TABLES}

1. Reactions during ignition and their effect on the relation between LOI and $\Sigma$ volatile components E2

2. Results of major rock-forming constituent analyses of SDO-1 E4

3. Volatile components as measured in SDO-1 E4

4. Calculations for correction of measured volatile components to account for ignition reactions $\mathbf{E 5}$

5. Results for rare earth elements in SDO-1 $\mathbf{E 5}$ 



\title{
Relation Between Volatile Components and Loss on Ignition as Applied to the Analysis of USGS Reference Devonian Ohio Shale SDO-1 and Rare Earth Element Analyses
}

\author{
By Miroslav Huka and Ivan Rubeska ${ }^{1}$
}

\begin{abstract}
In organic-rich shales, hydrogen bound to hydrocarbons is oxidized to water and measured together with constitutional water, $\mathrm{H}_{2} \mathrm{O}^{+}$. The amount of hydrogen released from the organic matter may be assessed from the difference between the experimental loss on ignition after correction for oxidation of ferrous iron and pyritic sulfur, and the sum of the experimentally measured volatile components, that is, sulfur, organic carbon $\left(\mathrm{C}_{\text {org }}\right)$, $\mathrm{CO}_{2}$, and the measured $\mathrm{H}_{2} \mathrm{O}^{+}$. This approach has been applied to the analytical data for the reference shale SDO-1 to improve the overall summation of major constituents, which should total $100 \pm 1$ percent. Results for the rare earth elements determined by inductively coupled plasma atomic emission spectrometry (ICP-AES), after two different separation procedures, are also presented. All results for SDO-1 in this report were included in the compiled data base from which recommended concentrations were derived.
\end{abstract}

\section{INTRODUCTION}

Chemical analysis of shales rich in organic matter for the major components included in a typical "silicate analysis" presents certain difficulties in achieving the expected summation to approximately 100 percent. These difficulties are caused mainly by the fact that oxygen, one of the main constituents, is not determined independently. Instead, the oxygen content is calculated on the basis of the assumptions that the standard oxide representation of the cations is correct and that the volatile components can be either accurately measured or reasonably estimated from loss on ignition (LOI). The relatively high content of organic carbon that usually occurs together with sulfide minerals in the shales has a deleterious influence on the results from

Manuscript approved for publication September 15, 1992.

${ }^{1}$ Geological Survey of Prague, Czechoslovakia several of the well-established procedures used in silicate analysis, invalidating these assumptions. Organic carbon and sulfide mineralization prevent accurate measurement of ferrous iron and $\mathrm{H}_{2} \mathrm{O}^{+}$and also affect the relation of $\mathrm{H}_{2} \mathrm{O}^{+}$, ferrous iron, sulfur, $\mathrm{C}_{\text {org }}$, and $\mathrm{CO}_{2}$ to LOI. However, if the relation of LOI to iron speciation and the volatile components can be well understood, the content of hydrogen bound in organic matter can be calculated for the final summation of the analysis. In this report, the LOI relation to other components is first discussed on a general level, and then the appropriate calculations are applied to data obtained for SDO-1 using common laboratory procedures for silicate rock analysis. All ignition reactions pertinent to the discussion that follows, the errors in LOI and total results that ignition reactions introduce, and appropriate corrections are summarized in table 1.

\section{RELATION OF VOLATILE COMPONENTS TO LOI}

\section{Normal Igneous Rocks}

In typical silicate rock analysis, LOI approximates the sum in the sample of volatile components, mainly $\mathrm{CO}_{2}$ expelled from carbonate, $\mathrm{S}, \mathrm{H}_{2} \mathrm{O}^{+}$, and halogens. Therefore, it should be possible to calculate one of the volatile components, $\mathrm{H}_{2} \mathrm{O}^{+}$, by difference from the rest and the measured LOI $\left(\mathrm{LOI}_{\text {exp }}\right)$. However, some of the sample constituents change during ignition, causing analytical error to be introduced in the estimation of volatile components from $\mathrm{LOI}_{\text {exp. }}$. Proper corrections must be introduced to obtain a corrected value, $\mathrm{LOI}_{\text {corr }}$, which will equal, rather than only approximate, the sum of the volatile components.

The effect on $\mathrm{LOI}_{\text {exp }}$ of oxidizing ferrous iron is a well-known example. Commonly, in the analysis of igneous rocks, the $\mathrm{LOI}_{\text {exp }}$ is decreased in comparison to $\mathrm{LOI}_{\text {corr }}$ by the weight gain from adding oxygen to the sample during 
Table 1. Reactions during ignition and their effect on the relation between LOI and $\Sigma$ volatile components

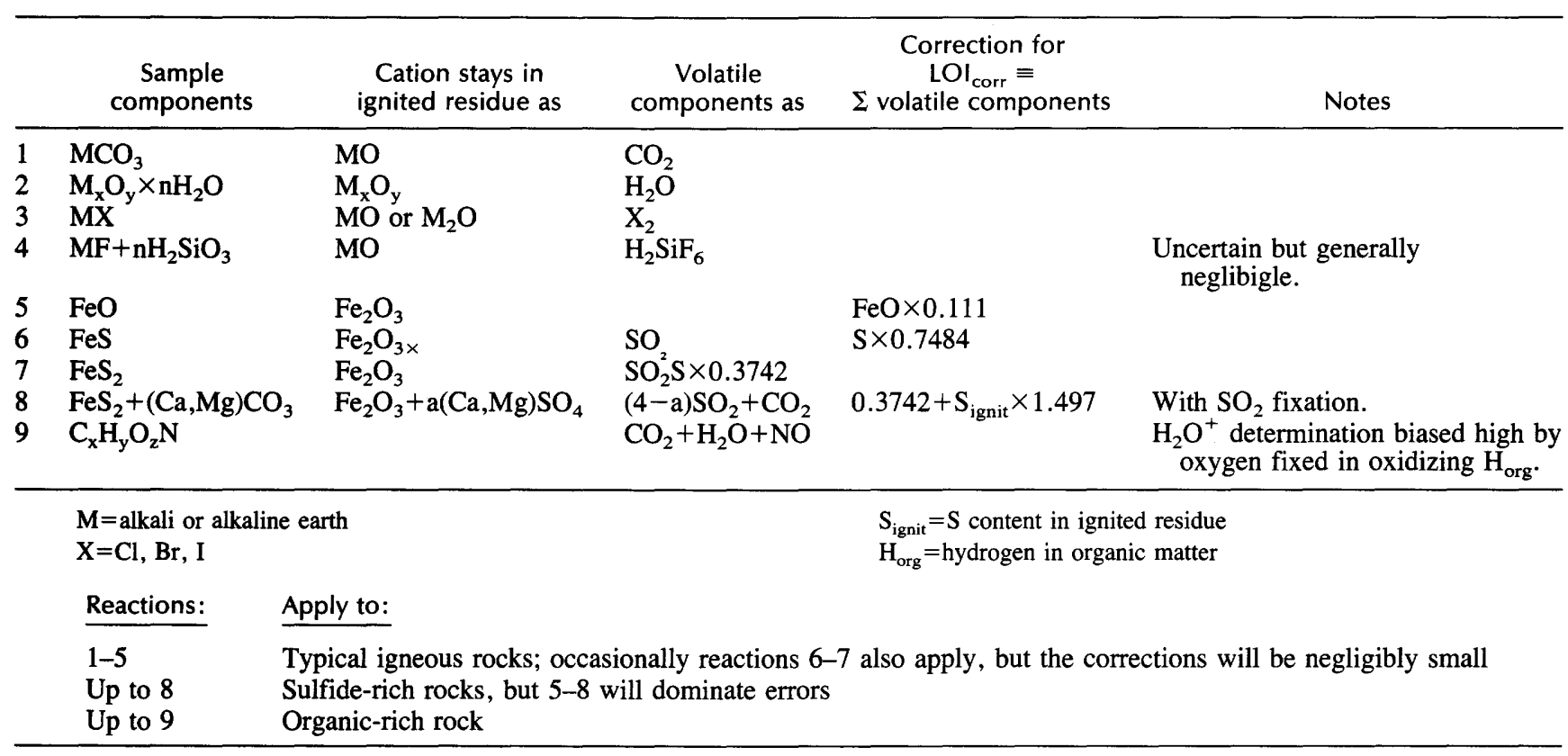

ignition, oxidizing divalent iron to trivalent. In the absence of sulfur, the correction is rather straightforward and may be expressed as

$$
\begin{aligned}
\mathrm{LOI}_{\text {corr }}=\mathrm{C} & +\mathrm{CO}_{2}+\mathrm{H}_{2} \mathrm{O}^{+}=\mathrm{LOI}_{\text {exp }}+\mathrm{FeO} \times 0.111 \\
=\Sigma \text { volatile components } &
\end{aligned}
$$

The multiplication factor 0.111 is the molecular mass ratio of $\mathrm{Fe}_{2} \mathrm{O}_{3}$ to $2 \mathrm{FeO}$ minus 1 , and the product expresses the mass increase of the ignited sample due to fixation of oxygen through oxidation of the originally divalent iron. After making this correction to $\mathrm{LOI}_{\text {exp }}$, the calculation of $\mathrm{H}_{2} \mathrm{O}^{+}$in "typical" igneous rocks from $\mathrm{LOI}_{\text {corr }}$ and the other volatile components can be made with considerable accuracy.

Also during ignition,the halogens $\mathrm{F}$ and $\mathrm{Cl}$ are partially lost from samples. At high ignition temperatures, when $\mathrm{H}_{2} \mathrm{O}^{+}$is released, pyrohydrolysis takes place, and $\mathrm{Cl}$ volatilizes as $\mathrm{HCl}$. The process is supported by the $\mathrm{SiO}_{2}$ present, which acts as a nonvolatile acid. Fluorine behaves in a similar way, but the HF formed reacts immediately with $\mathrm{SiO}_{2}$ and volatilizes as $\mathrm{SiF}_{4}$ or $\mathrm{H}_{2} \mathrm{SiF}_{6}$. The losses are greater for acid rocks than for basic ones. In most cases, the error in $\mathrm{SiO}_{2}$ affecting the final analytical summation is negligible, and inclusion of halogens in the sum of volatile components is sufficient in obtaining a value for $\mathrm{LOI}_{\text {corr }}$ from which a calculated $\mathrm{H}_{2} \mathrm{O}^{+}$can be accurately derived.

It was noted above that $\mathrm{FeO}$, normally measured straightforwardly in "typical" silicate rock analysis, cannot be measured directly in the presence of organic matter. Also ferrous iron in pyrite is not measured using the standard ferrous iron procedure. Obtaining $\mathrm{LOI}_{\text {corr }}$ for SDO-1, then, is not straightforward; the remainder of this section examines the problem.

\section{The Effect of Sulfidic Sulfur}

If sulfur is present in appreciable amount $(>0.2$ percent), the error in $\mathrm{LOI}_{\exp }$ becomes more complicated. Most often the sulfur in shales occurs bound to iron either as pyrite/marcasite $\left(\mathrm{FeS}_{2}\right)$ or as pyrrhotite $(\mathrm{FeS})$. However, all iron is typically reported as $\mathrm{Fe}_{2} \mathrm{O}_{3}$ in rock analyses. This misrepresentation of iron speciation in the final summation of oxides results in a positive numerical error since both the sulfur actually bound to iron and the "sulfur equivalent" oxygen (SOQ) reportedly bound to iron are included. The oxide summation is corrected by subtracting SOQ from the initial summation. For pyrite the SOQ is $S \times 0.25$ $(16 / 2 \times 32.06)$, whereas for pyrrhotite it is $S \times 0.5$ (16/32.06). Thus, accurate correction requires knowledge of the mode of sulfur occurrence in the sample.

It is possible to differentiate between the pyrite and pyrrhotite by showing differences in the solubilities of the two materials or by using a qualitative test; the presence of pyrrhotite is revealed by the smell of $\mathrm{H}_{2} \mathrm{~S}$ when adding acid to the sample. A common-sense judgment can also be used, based on the fact that pyrrhotite generally occurs in basic rocks, whereas pyrite and marcasite are common in shales. Further discussion will therefore be limited to considering occurrences of pyrite in the sample.

In addition to the SOQ correction in the major oxide total, a correction of $\mathrm{LOI}_{\text {exp }}$ must also be introduced for the oxygen mass added to oxidize pyritic (divalent) iron to 
$\mathrm{Fe}_{2} \mathrm{O}_{3}$. This oxygen increases the mass of the ignited sample and reduces $\mathrm{LOI}_{\exp }$ in comparison to $\mathrm{LOI}_{\text {corr }}$, as already discussed for samples containing ferrous oxide. Assuming that the sulfur is bound more or less exclusively to iron, this correction is made by multiplying the sulfur content by 0.3742 :

$$
\mathrm{LOI}_{\text {corr }}=\mathrm{S}+\mathrm{CO}_{2}+\mathrm{H}_{2} \mathrm{O}^{+}=\mathrm{LOI}_{\text {exp }}+(\mathrm{S} \times 0.3742) .
$$

However, this correction might be inadequate if the sample contains $\mathrm{CaCO}_{3}$ or $\mathrm{MgCO}_{3}$, either of which can fix $\mathrm{S}$ in the ignited sample as sulfate. The direct determination of $\mathrm{CO}_{2}$ as one of the volatiles in the sample gives an indication of the potential magnitude of this problem. Equation 2 also neglects oxidation of any ferrous iron in addition to pyritic iron. To account for both sulfur fixation and that oxidation, equation 2 becomes

$$
\begin{gathered}
\mathrm{LOI}_{\text {corr }}=\left(\mathrm{S}-\mathrm{S}_{\text {ignit }}\right)+\mathrm{CO}_{2}+\mathrm{H}_{2} \mathrm{O}^{+} \\
=\mathrm{LOI}_{\text {exp }}+\mathrm{FeO} \times 0.111+\mathrm{S} \times 0.3742+\mathrm{S}_{\text {ignit }} \times 1.497 .
\end{gathered}
$$

\section{The Effect of Inherent Sulfate Occurrences on LOI}

Although the discussion above assumed that essentially all sulfur in the sample occurred as pyrite or pyrrhotite, sulfur may also be present in the sample initially as sulfate. Sulfates of alkali and alkaline earth elements are unaltered during ignition, whereas sulfates of heavy or trivalent metals (for example, $\mathrm{Fe}, \mathrm{Mn}, \mathrm{Cu}$ ) decompose, releasing $\mathrm{SO}_{3}$. Oxidation of sulfide to sulfate is a common natural geochemical process and may even take place during storage of the sample in the laboratory, particularly for samples containing considerable $\mathrm{H}_{2} \mathrm{O}^{-}$stored at high temperatures.

The presence of alkaline earth sulfates in the sample generally results in an analytical summation low by an amount equal to the excess oxygen in sulfate compared to oxide, $S_{\text {sulf }} \times 1.497$. The presence of alkaline earth sulfates, however, does not cause an error in the estimation of $\mathrm{H}_{2} \mathrm{O}^{+}$ from equations 2 or 3 . On the contrary, the presence of heavy metal sulfates would overestimate $\mathrm{H}_{2} \mathrm{O}^{+}$, using those equations, by the excess of oxygen in sulfate compared to oxide, that is, by $S_{\text {sulf }} \times 1.497$.

\section{The Effect of Organic Carbon on LOI}

During ignition to determine $\mathrm{LOI}_{\text {exp }}$, all organic matter in the sample is oxidized and volatilized:

$$
\begin{gathered}
\text { Volatile components } \\
=(\mathrm{CHON})_{\text {org }}+\mathrm{S}+\mathrm{CO}_{2}+\mathrm{H}_{2} \mathrm{O}_{\text {true }}^{+}
\end{gathered}
$$

The ignition should proceed slowly at a temperature that avoids melting of the sample; otherwise, incomplete oxidation of carbon and partial reduction of $\mathrm{Fe}_{2} \mathrm{O}_{3}$ to $\mathrm{FeO}$ might occur in the molten sample. Either would result in a low $\mathrm{LOI}_{\text {exp }}$, and an error in the calculation of $\mathrm{H}_{2} \mathrm{O}^{+}$.

Assuming that complete oxidation of organic matter also occurs during determination of $\mathrm{H}_{2} \mathrm{O}^{+}$in organic-rich samples, the measured $\mathrm{H}_{2} \mathrm{O}^{+}$will be higher than the amount calculated from LOI and the other individual volatile components (eq. 2). This difference occurs because organic hydrogen will be oxidized to water and included in the measured $\mathrm{H}_{2} \mathrm{O}^{+}$result. The excess water can be used to estimate the hydrogen bound in hydrocarbons:

$$
\mathrm{H}_{2} \mathrm{O}^{+}{ }_{\text {meas }}-\mathrm{H}_{2} \mathrm{O}^{+}{ }_{\text {calc }}=9 \times \mathrm{H}_{\text {org }} \text {. }
$$

Further, the calculated value can be used to approximate the true constitutional water in the sample, which cannot be as accurately measured in organic-rich samples as in "typical" rocks.

\section{EXPERIMENT PARAMETERS}

The SDO-1 sample was analyzed on an "as received" basis, except for $\mathrm{LOI}_{\text {exp }}$, which was determined on the dry sample. Duplicate analyses from each of two bottles of SDO-1 were made using methods routinely applied in the authors' laboratory (Weiss, 1983; Huka and Rubeska, in press). The samples were analyzed as part of a batch $(\sim 200)$ of routine samples. The major oxides that are required for the analytical summation were determined from a single decomposition of the sample using a mixture of $\mathrm{HF}$, $\mathrm{HNO}_{3}$, and $\mathrm{H}_{2} \mathrm{SO}_{4}$. A 0.200 -g sample was decomposed in a specially designed polytetrafluoroethylene vessel assembly where the evolving $\mathrm{SiF}_{4}$ was sorbed on $\mathrm{KNO}_{3}$. After hydrolysis of the resulting $\mathrm{K}_{2} \mathrm{SiF}_{6}$, $\mathrm{Si}$ was determined by titrating the released $\mathrm{HF}$ with $\mathrm{NaOH}$ (Huka and Rubeska, in press). Fluorides in the sample residue were removed by fuming after the addition of $\mathrm{H}_{3} \mathrm{BO}_{3}$ and $\mathrm{HNO}_{3}$. About 50 $\mathrm{mL}$ of distilled water were added to the dry residue, and the sample was left on a warm plate to hydrolyze for 20 to 30 minutes. Then, $10 \mathrm{~mL} \mathrm{1:1} \mathrm{HCl}$, with $0.2 \mathrm{~g} \mathrm{Cs}_{2} \mathrm{CO}_{3}$ and $0.25 \mathrm{~mL} \mathrm{H}_{2} \mathrm{SO}_{4}$ as an ionization buffer, were added. The resulting clear solution was transferred to a $100-\mathrm{mL}$ volumetric flask and made up to volume with distilled water. $\mathrm{Li}$, $\mathrm{Na}, \mathrm{K}, \mathrm{Mg}, \mathrm{Ca}, \mathrm{Ti}$, and $\mathrm{Mn}$ were measured by flame atomic absorption spectrometry (Fl-AAS). $\mathrm{Fe}_{\text {tot }}$ and $\mathrm{P}_{2} \mathrm{O}_{5}$ were determined by spectrophotometry, using the sulfosalicylic acid and molybdenum blue procedures, respectively. Al was determined by the Sajo (Weiss, 1983) chelatometric method with ethylenediamine tetraacetic acid.

Moisture $\left(\mathrm{H}_{2} \mathrm{O}^{-}\right)$was determined to be 0.79 weight percent by drying the weighed sample at $110^{\circ} \mathrm{C}$ for 4 hours and reweighing. LOI was determined on the dry sample, 
Table 2. Results of major rock-forming constituent analyses of SDO-1

[In weight percent]

\begin{tabular}{lcccc}
\hline $\begin{array}{c}\text { Split: } \\
\text { Component }\end{array}$ & $393 \mathrm{a}$ & $393 \mathrm{~b}$ & $768 \mathrm{a}$ & $768 \mathrm{~b}$ \\
\hline $\mathrm{SiO}_{2}$ & 49.43 & 49.29 & 49.62 & 49.26 \\
$\mathrm{Al}_{2} \mathrm{O}_{3}$ & 12.06 & 12.13 & 12.10 & 12.03 \\
$\mathrm{Fe}_{2} \mathrm{O}_{3}$ & 3.03 & 2.98 & 3.04 & 3.07 \\
$\mathrm{FeO}$ & 5.64 & 5.66 & 5.59 & 5.63 \\
$\mathrm{MnO}$ & .041 & .040 & .040 & .042 \\
$\mathrm{MgO}$ & 1.47 & 1.46 & 1.48 & 1.49 \\
$\mathrm{CaO}$ & 1.01 & 1.07 & 1.08 & 1.06 \\
$\mathrm{Li} 2 \mathrm{O}$ & .006 & .006 & .006 & .006 \\
$\mathrm{Na}_{2} \mathrm{O}$ & .45 & .43 & .43 & .42 \\
$\mathrm{~K}_{2} \mathrm{O}$ & 3.24 & 3.23 & 3.23 & 3.22 \\
$\mathrm{P}_{2} \mathrm{O}_{5}$ & .01 & .01 & .01 & .01 \\
\hline
\end{tabular}

which was ignited at $1,050^{\circ} \mathrm{C}$ for 20 minutes and reweighed. Carbonate was measured by the release of $\mathrm{CO}_{2}$ from the sample by boiling with $\mathrm{H}_{3} \mathrm{PO}_{4}$ and its sorption on $\mathrm{Ba}(\mathrm{OH})_{2}$; the sorbed $\mathrm{CO}_{2}$ was titrated using a Stroehlein Coulomat Model 7012. Total carbon was determined from the $\mathrm{CO}_{2}$ released by igniting the sample in a stream of oxygen, measured as just described for $\mathrm{CO}_{2}$ in the sample. Sulfur was determined after its release as $\mathrm{SO}_{3}$ by heating the sample with a catalyst, $\mathrm{V}_{2} \mathrm{O}_{5}+\mathrm{U}_{3} \mathrm{O}_{8}$, in a stream of nitrogen. After sorption, the $\mathrm{SO}_{3}$ was titrated with coulometrically generated iodine, using biamperometric indication of the endpoint.

Rare earth analyses were performed by inductively coupled plasma atomic emission spectrometry following two different procedures for the separation of the rare earth elements (REE) from the sample matrix. One using ionexchange chromatography was described by Sulcek and others (1989); the second involved an organic solvent extraction of ion associates of REE nitrates with trioctylphosphine oxide (Weiss and others, 1990).

\section{RESULTS AND DISCUSSION}

Results for major-oxide analyses of SDO-1 are presented in table 2. Results for the volatile components that were measured directly are presented in table 3 . Using the logic of the preceding section describing the potential analytical errors in analytical summation and LOI (summarized in table 1), corrections for those errors were made, as follows.

Since it was not possible to determine $\mathrm{FeO}$ directly in the presence of organic matter, the assumption was made that all $\mathrm{S}$ present occurred in pyrite and that a corresponding stoichiometric amount of $\mathrm{Fe}^{+2}$ occurred in the sample. The balance of the total iron was assumed to be ferric iron. Little carbonate was measured in SDO-1; thus, no appreciable
Table 3. Volatile components as measured in SDO-1 [In weight percent]

\begin{tabular}{lrrrr}
\hline $\begin{array}{c}\text { Split: } \\
\text { Component }\end{array}$ & $393 \mathrm{a}$ & $393 \mathrm{~b}$ & $768 \mathrm{a}$ & $768 \mathrm{~b}$ \\
\hline $\mathrm{F}$ & $<0.01$ & $<0.01$ & $<0.01$ & $<0.01$ \\
$\mathrm{C}_{\text {tot }}$ & 10.41 & 10.40 & 10.48 & 10.57 \\
$\mathrm{CO}_{2}$ & .72 & .70 & .81 & .84 \\
$\mathrm{C}_{\text {org }}=\mathrm{C}_{\text {tot }}-\mathrm{C}_{\mathrm{CO}^{2}}$ & 10.21 & 10.21 & 10.26 & 10.35 \\
$\mathrm{~S}$ & 5.04 & 5.05 & 4.99 & 5.03 \\
$\mathrm{H}_{2} \mathrm{O}_{\text {meas }}^{+}$ & 9.66 & 9.46 & 9.53 & 9.28 \\
Volatile components & 25.63 & 25.42 & 25.59 & 25.49 \\
LOI $_{\text {exp }}$ & 21.59 & 21.45 & 21.56 & 21.51 \\
\hline
\end{tabular}

amount of $S$ is expected to be fixed in the ignited residue as sulfate. $\mathrm{LOI}_{\text {corr }}$ from equation 3 is therefore reduced to

$$
\mathrm{LOI}_{\text {corr }}=\mathrm{LOI}_{\text {exp }}+\mathrm{S} \times 0.3742 \text {, }
$$

which accounts for oxidation of pyrite to hematite.

From this calculated $\mathrm{LOI}_{\text {corr }}$, values for volatile components, $\mathrm{H}_{\text {org }}$, and true $\mathrm{H}_{2} \mathrm{O}^{+}$are derived. Table 4 summarizes these calculations and presents the analytical summation for the analyses, based on those calculations and the major-oxide analyses presented in table 2 . The totals are not ideal, due to the uncertainties involved in the approach. However, as can be seen, the approach improves the uncorrected totals significantly.

The REE data by ICP-AES following the two separations are presented in table 5. They agree well with recommended concentrations (Kane and others, 1990) that were derived from these and other contributed data. However, the extraction procedure apparently provides higher results than those obtained using the ion-exchange procedure. This difference probably occurs because, for the extraction, calibration solutions were carried through the separation; whereas for ion exchange, they were not. An ion- exchange yield of less than 100 percent would cause a low bias in the sample analyses. No such bias would occur if extraction yields were less than 100 percent, but constant for sample and calibration solutions.

\section{CONCLUSION}

The results presented in this paper formed a part of the compiled data base from which the recommended concentrations were derived for the gas shale reference sample SDO-1. The methods used in our laboratory produce analytical results in excellent agreement with the final recommended concentrations for both major oxides and rare earth elements. Applying the described corrections to LOI provides acceptable major-oxide summations for the analysis of organic-rich and sulfur-rich samples, which are generally quite difficult to achieve for such samples. 
Table 4. Calculations for correction of measured volatile components to account for ignition reactions

\begin{tabular}{|c|c|c|}
\hline Calculation & $\begin{array}{c}\text { Result } \\
\text { in weight percent }\end{array}$ & Comment \\
\hline $\mathrm{SOQ}=(\mathrm{S})(0.25)$ & -1.26 & $\begin{array}{l}\text { Oxygen equivalent of sulfur for pyritic iron represented } \\
\text { as FeO in major oxide table } 1 \text {; see text E2. }\end{array}$ \\
\hline $\mathrm{LOI}_{\text {corr }}=\mathrm{LOI}_{\text {exp }}+(\mathrm{S})(0.3742)$ & 23.43 & Oxygen added in oxidizing pyrite to $\mathrm{Fe}_{2} \mathrm{O}_{3}$; see eq. 2 . \\
\hline $\mathrm{LOI}_{\text {corr }}=\mathrm{S}+\mathrm{C}_{\text {org }}+\mathrm{CO}_{2}+\mathrm{H}_{2} \mathrm{O}_{\text {meas }}^{+}$ & 25.54 & Alternative calculation to that above; see eq. 3 . \\
\hline excess $\mathrm{O}=$ eq. $3 \mathrm{LOI}_{\text {corr }}-$ eq. $2 \mathrm{LOI}_{\text {corr }}$ & 2.11 & Fixed on oxidation of organic $\mathrm{H}$; see text E3. \\
\hline $\mathrm{H}_{\text {org }}=$ excess $\mathrm{O} \div 8$ & .27 & $\mathrm{H}_{2} \mathrm{O}$ graumetric factor. \\
\hline excess $\mathrm{H}_{2} \mathrm{O}^{+}=$excess $\mathrm{O}+\mathrm{H}_{\text {org }}$ & 2.38 & \\
\hline $\mathrm{H}_{2} \mathrm{O}_{\text {true }}^{+}=\mathrm{H}_{2} \mathrm{O}_{\text {meas }}^{+}-$excess $\mathrm{H}_{2} \mathrm{O}^{+}$ & 7.12 & See text E3. \\
\hline Major oxide summation & 76.29 & Table 1 , average \\
\hline $\mathrm{CO}_{2}, \mathrm{C}_{\text {org }}, \mathrm{S}$ summation & 16.06 & Unbiased measured volatiles, table 2 . \\
\hline $\mathrm{H}_{2} \mathrm{O}^{-}$ & .79 & Moisture; see text E3. \\
\hline $\mathrm{H}_{2} \mathrm{O}_{\text {true }}^{+}$ & 7.12 & Calculated above. \\
\hline $\mathrm{H}_{\text {org }}$ & .27 & Calculated above. \\
\hline SOQ & -1.26 & Calculated above. \\
\hline Analytical summation, whole rock & 100.53 & Meets $100 \pm 1$ test for overall analytical accuracy. \\
\hline
\end{tabular}

Table 5. Results for rare earth elements in SDO-1

[In $\mu \mathrm{g} / \mathrm{g} ; \mathrm{NA}$, not analyzed]

\begin{tabular}{lcccc}
\hline & \multicolumn{2}{c}{ lon exchange } & & \multicolumn{2}{c}{ Extraction } \\
\cline { 2 - 3 } \cline { 5 - 6 } $\begin{array}{c}\text { Split: } \\
\text { Element }\end{array}$ & 393 & 768 & 393 & 768 \\
\hline $\mathrm{La}$ & & & & \\
$\mathrm{Ce}$ & 35.3 & 34.7 & 38.6 & 37.3 \\
$\mathrm{Pr}$ & 71.6 & 70.9 & 86.1 & 84.1 \\
$\mathrm{Nd}$ & 8.00 & 7.79 & 8.55 & 8.75 \\
$\mathrm{Sm}$ & 35.3 & 36.0 & 35.5 & 35.9 \\
$\mathrm{Eu}$ & 7.71 & 7.36 & 8.47 & 7.91 \\
$\mathrm{Gd}$ & 1.46 & 1.48 & 1.65 & 1.69 \\
$\mathrm{~Tb}$ & 7.06 & 7.11 & 8.40 & 7.80 \\
$\mathrm{Dy}$ & .81 & 1.04 & .91 & 1.01 \\
$\mathrm{Ho}$ & 6.22 & 6.33 & 7.14 & 7.08 \\
$\mathrm{Er}$ & 1.22 & 1.10 & 1.31 & 1.46 \\
$\mathrm{Tm}$ & 3.83 & 3.00 & 4.00 & 3.96 \\
$\mathrm{Yb}$ & .49 & .50 & $<.40$ & $<.40$ \\
$\mathrm{Lu}$ & 3.05 & 3.11 & 3.80 & 3.80 \\
$\mathrm{Y}$ & .49 & .49 & .55 & .55 \\
$\mathrm{Sc}$ & 34.8 & 35.0 & 40.6 & 40.2 \\
\hline & 13.0 & 12.5 & $\mathrm{NA}$ & $\mathrm{NA}$ \\
\hline
\end{tabular}

\section{REFERENCES}

Huka, Miroslav, and Rubeska, Ivan, in press, An expeditious wet chemical procedure for silicate analysis with titrimetric determination of silica: Geoanalysis 90 Symposium volume, Geological Survey of Canada Paper.

Kane, J.S., Arbogast, B.F., and Leventhal, J.S., 1990, Characterization of Devonian Ohio Shale SDO-1 as a USGS geochemical reference sample: Geostandards Newsletter, v. 14, p. 169-196.

Sulcek, Zdenek, Rubeska, Ivan, Sixta, Vaclav, and Paukert, Tomas, 1989, Determination of REE and Y in rocks using the Plasma 2 ICP emission spectrometer: Atomic Spectroscopy, v. 10, p. 4-9.

Weiss, Dalibor, ed., 1983, Methods of chemical analysis of the Geological Survey of Prague.

Weiss, Dalibor, Paukert, Tomas, and Rubeska, Ivan, 1990, Determination of REE and $Y$ in rocks by ICP-AES after separation by organic solvent extraction: Journal of Analytical Atomic Spectrometry, v. 5, p. 371-375. 

Chapter F

A Study to Determine Sources of Interlaboratory Variability in Measured Loss on Ignition (LOI) for Devonian Ohio Shale SDO-1

By JEAN S. KANE and CAROL J. SKEEN 



\title{
CONTENTS
}

\author{
Abstract F1 \\ Introduction F1 \\ Review of compiled data for $\mathrm{FeO}$ and Volatile Components F1 \\ Experiment Parameters F3 \\ Moisture $\left(\mathrm{H}_{2} \mathrm{O}^{-}\right)$and LOI F3 \\ Determination of Ferrous Iron $\mathbf{F 4}$ \\ Measurement of CHNS F4 \\ Results and Discussion F4 \\ Moisture $\left(\mathrm{H}_{2} \mathrm{O}^{-}\right) \quad$ F4 \\ LOI F5 \\ $\mathrm{FeO}$ and $\mathrm{S}$ in Ignited Sample $\mathbf{F 5}$ \\ CHN in Both "As Received" and Ignited Sample F6 \\ Analytical Total F6 \\ Conclusion $\mathbf{F 8}$ \\ References $\mathbf{F 8}$
}

\section{FIGURES}

1, 2. Graphs showing:

1. Moisture results after cyclic drying and reabsorption F5

2. LOI as a function of temperature, illustrating interaction between temperature and bottle components of variance $\mathbf{F 8}$

\section{TABLES}

1. Compiled data for volatile components F2

2. Moisture: Evaluation of bottle differences F4

3. Loss on ignition: Evaluation of bottle and ignition-temperature differences F6

4. Reactions during ignition of sample F6

5. $\mathrm{FeO}$ and $\mathrm{S}$ in ignited sample residue and weight gain calculations $\mathbf{F} 7$

6. CHN results for SDO-1 F7

7. Alternative estimates of the sum of volatile components 



\title{
A Study to Determine Sources of Interlaboratory Variability in Measured Loss on Ignition (LOI) for Devonian Ohio Shale SDO-1
}

\author{
By Jean S. Kane and Carol J. Skeen
}

\begin{abstract}
Studies are reported that investigate the several components in the sizable (approximately 10 percent relative) variance between laboratories in measuring loss on ignition for the reference sample Devonian Ohio Shale SDO-1. This large variance is a major factor in the poor analytical summation for the major constituents of the shale that was noted in the original compilation report (Kane and others, 1990).
\end{abstract}

\section{INTRODUCTION}

In reporting recommended concentrations for constituents in the reference shale sample SDO-1, difficulty was encountered in verifying the overall SDO- 1 analyses using a major-oxide summation to $100 \pm 1$ percent (Kane and others, 1990). Such verification is very common in rock analysis (Abbey, 1983; Lechler and Desilets, 1987), but it is less applicable to the analysis of either organic-rich or sulfur-rich samples. For example, totals of the recommended major-oxide concentrations (including estimated volatile components) for marine mud (MAG-1), Cody Shale (SCo-1), and Green River Shale (SGR-1) reference samples are $105.5,100.35$, and 88.82 percent, respectively (Gladney and Roelandts, 1988).

The difficulty results from the inability to measure individual volatile components accurately and from the considerable error that results from using measured loss on ignition (LOI) as a measure of the sum of volatile components (Lechler and Desilets, 1987; Potts, 1987; Huka and Rubeska, chap. E, this vol.). The important volatile components are water (both moisture, $\mathrm{H}_{2} \mathrm{O}^{-}$, and essential water, $\left.\mathrm{H}_{2} \mathrm{O}^{+}\right)$, carbon dioxide $\left(\mathrm{CO}_{2}\right)$, sulfur $(\mathrm{S})$, and organic matter. Only $\mathrm{H}_{2} \mathrm{O}^{-}, \mathrm{CO}_{2}$, and $\mathrm{S}$ can be measured directly.

In most rock analyses, total water $\left(\mathrm{H}_{2} \mathrm{O}_{\text {tot }}\right)$ and moisture $\left(\mathrm{H}_{2} \mathrm{O}^{-}\right)$are measured, whereas essential, or

\footnotetext{
Manuscript approved for publication September 15, 1992.
}

bound, water $\left(\mathrm{H}_{2} \mathrm{O}^{+}\right)$is calculated by difference. For organic-rich samples, $\mathrm{H}_{2} \mathrm{O}_{\text {tot }}$ as measured includes both the $\mathrm{H}_{2} \mathrm{O}^{+}$in sample and the water resulting from combustion of organic matter. $\mathrm{H}_{2} \mathrm{O}^{+}$calculated in the normal way is biased high by the organic matter contribution to $\mathrm{H}_{2} \mathrm{O}^{+}$.

Although total carbon $\left(\mathrm{C}_{\mathrm{tot}}\right)$ and $\mathrm{CO}_{2}$ can be measured readily, $\mathrm{C}_{\text {org }}$ is most often determined by difference between $\mathrm{C}_{\text {tot }}$ and $\mathrm{C}_{\text {carbonate }}\left(\mathrm{CO}_{2} \times 0.274\right)$. From $\mathrm{C}_{\text {org }}$, total organic matter can be estimated, but uncertainties are considerable. Ratios of $\mathrm{C}$ to $\mathrm{H}$ in organic matter (as determined by $\mathrm{CHN}$ analysis of organic matter isolated from shale by Soxlet extraction) are biased low, overestimating $\mathrm{H}_{\text {org }}$ (Frost and others, 1985). This error occurs because low-molecular-weight organic compounds are preferentially extracted. Correction of $\mathrm{H}_{2} \mathrm{O}_{\text {tot }}$ for the $\mathrm{H}_{\text {org }}$ contribution will be correspondingly inexact.

Frost and others (1985) achieved totals generally between 98 and 101 percent, but occasionally as low as 96 percent or as high as 103 percent, for the analyses of a large number $(\sim 250)$ of Devonian shales from Illinois. They assumed that $\mathrm{H}_{2} \mathrm{O}^{+}$could be fairly accurately estimated from a hydrogen mass balance after measuring $\mathrm{H}_{\text {tot }}$ and moisture and calculating an organic hydrogen value. Huka and Rubeska (chap. E, this vol.) present another approach to achieving an accurate analytical summation by estimating $\mathrm{H}_{2} \mathrm{O}^{+}$from the experimental loss on ignition ( $\mathrm{LOI}_{\text {exp }}$ ). They outline corrections to $\mathrm{LOI}_{\text {exp }}$ that are required for $\mathrm{LOI}$ to approximate more accurately the sum of volatile components in sample. This paper reviews the compiled data relevant to LOI and presents the experiments undertaken in our laboratory to better understand the various components of uncertainty in LOI.

\section{REVIEW OF COMPILED DATA FOR FEO AND VOLATILE COMPONENTS}

Table 1 includes all previously contributed and newly reported data for LOI, ferrous iron, and individual volatile components, and for moisture, $\mathrm{H}$, and $\mathrm{N}$. The moisture, $\mathrm{H}$, 
Table 1. Compiled data for volatile components

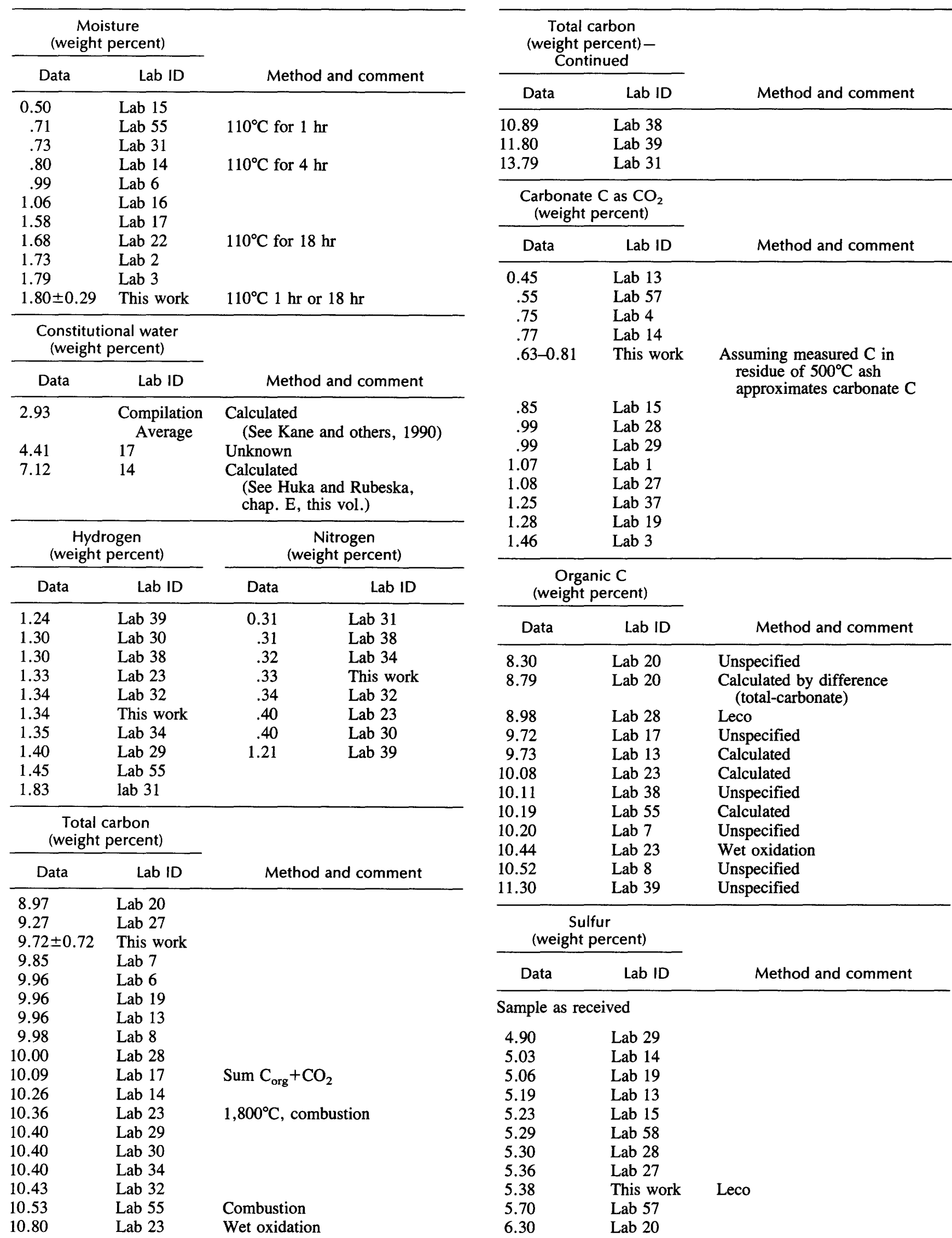


Table 1. Compiled data for volatile componentsContinued

\begin{tabular}{|c|c|c|}
\hline \multicolumn{2}{|c|}{$\begin{array}{c}\text { Sulfur } \\
\text { (weight percent)- } \\
\text { Continued }\end{array}$} & \multirow[b]{2}{*}{ Method and comment } \\
\hline Data & Lab ID & \\
\hline \multicolumn{3}{|c|}{ Ignited sample } \\
\hline 0.71 & Lab 21 & XRF \\
\hline .43 & This work & Leco, $1,000^{\circ} \mathrm{C}$ \\
\hline 1.41 & This work & Leco, $500^{\circ} \mathrm{C}$ \\
\hline \multicolumn{2}{|c|}{$\begin{array}{l}\text { Loss on ignition } \\
\text { (weight percent) }\end{array}$} & \multirow[b]{2}{*}{ Method and comment } \\
\hline Data & Lab ID & \\
\hline 19.25 & This work & $500^{\circ} \mathrm{C}, 1 \mathrm{hr}$ \\
\hline 19.43 & This work & $500^{\circ} \mathrm{C}, 18 \mathrm{hr}$ \\
\hline 19.56 & Lab 3 & \\
\hline 19.56 & Lab 1 & $550^{\circ} \mathrm{C}, 1 \mathrm{hr}$ \\
\hline 19.78 & Lab 27 & \\
\hline 20.82 & This work & $800^{\circ} \mathrm{C}, 1 \mathrm{hr}$ \\
\hline 20.83 & Lab 13 & \\
\hline 20.91 & Lab 18 & \\
\hline 21.37 & Lab 58 & \\
\hline 21.38 & Lab 16 & \\
\hline 21.57 & Lab 6 & \\
\hline 21.63 & Lab 4 & $1,000^{\circ} \mathrm{C}, 1 \mathrm{hr}$ \\
\hline 21.82 & Lab 17 & \\
\hline 21.90 & Lab 21 & \\
\hline 21.93 & Lab 15 & \\
\hline 22.00 & Lab 57 & \\
\hline 22.35 & Lab 26 & \\
\hline 22.37 & Lab 19 & \\
\hline 22.39 & Lab 14 & $1,050^{\circ} \mathrm{C}, 20 \mathrm{~min}$ \\
\hline 22.84 & Lab 2 & \\
\hline 23.06 & This work & $1,000^{\circ} \mathrm{C}, 1 \mathrm{hr}$ \\
\hline 23.08 & Lab 22 & $1,000^{\circ} \mathrm{C}, 1 \mathrm{hr}$ \\
\hline 33.00 & Lab 20 & \\
\hline
\end{tabular}

and $\mathrm{N}$ data were not included in the 1990 compilation (Kane and others).

An examination of the compiled data reveals four points. First is the general lack of data for individual volatile components in comparison to major oxides, each of which was reported by 20 to 30 contributors. Second is that LOI, $\mathrm{CO}_{2}$, and $\mathrm{H}_{2} \mathrm{O}^{-}$all show an unacceptably large variation between laboratories. For LOI, fewer than half the contributing laboratories reported ignition temperature, which significantly affects experimental LOI. For example, the omission of the obvious outlier value of 33-percent LOI results in the 3.5-percent absolute difference between the high and low laboratory average LOI. This variation results from several factors, one of which is the significant between-bottle variation for LOI noted in the 1990 compilation (Kane and others). Five of eight laboratories identified between-bottle variance, which may be equally significant for $\mathrm{H}_{2} \mathrm{O}^{-}$and $\mathrm{CO}_{2}$, although the data reported does not permit assessment of that possibility.
Variation of ignition temperature is the third factor contributing to between-laboratory variance. Laboratories reported using various ignition temperatures from $500^{\circ} \mathrm{C}$ to $1,000^{\circ} \mathrm{C}$. Although ignition to constant weight occurs within 1 hour at $1,000^{\circ} \mathrm{C}$ (Lechler and Desilets, 1987), it proceeds more slowly at lower temperatures. Therefore ignition at $500^{\circ} \mathrm{C}$ for one hour is incomplete and the LOI result is biased low. This factor will be treated more fully in the results and discussion section.

Also, few laboratories indicated whether or not samples were ignited "as received" or after drying. If $\mathrm{H}_{2} \mathrm{O}^{-}$was not reported separately, LOI was assumed to have been reported on an "as received" basis so that the final recommended LOI result includes $\mathrm{H}_{2} \mathrm{O}^{-}$along with all other volatile components. Similarly, if $\mathrm{H}_{2} \mathrm{O}^{-}$was reported separately, it was assumed that the reported LOI did not include moisture and that value was added, so that LOI as compiled would include it. If either (or both) of these assumptions is incorrect, that would account for a part of the observed between-laboratory variance in LOI results.

The final point to note regarding the compiled data in table 1 is that LOI as measured does not accurately reflect the sum of volatile components (Lechler and Desilets, 1987; Huka and Rubeska, chap. E, this vol.; Potts, 1987). Therefore, LOI cannot be used with the major rock-forming oxides in achieving an accurate analytical summation. For typical rock analysis, the inaccuracy is due almost exclusively to the weight gain resulting from oxidation of ferrous oxide to ferric oxide (Lechler and Desilets, 1987). Since $\mathrm{FeO}$ and total iron can both be measured directly and accurately for most rocks, correction is straightforward. For sulfur-rich and organic-rich sedimentary rocks (and SDO-1 is both), the error is of similar origin-oxidation of both pyritic iron and ferrous oxide to ferric oxide. Correction for this error is complicated in two ways. First is the inability to measure ferrous iron directly in the original sample due to interferences in the redox reactions for that measurement by both organic matter and sulfur. Second is an additional error in measured LOI due to the weight gain resulting from fixation of some sulfur as sulfate in the ignited sample. Correction for that error requires measurement of sulfur in the ignited sample; only one laboratory initially reported that measurement.

This study focused on establishing the significance of between-bottle differences for $\mathrm{H}_{2} \mathrm{O}^{-}, \mathrm{CO}_{2}$, and organic matter in sample and on the degree of ferrous iron oxidation and sulfur fixation during ignition.

\section{EXPERIMENT PARAMETERS}

\section{Moisture $\left(\mathrm{H}_{2} \mathrm{O}^{-}\right)$and LOI}

Both $\mathrm{H}_{2} \mathrm{O}^{-}$and LOI were measured following the standard procedures of the laboratory (Jackson and others, 
1987; Shapiro and Brannock, 1962), which involve overnight drying at $105^{\circ} \mathrm{C}$ and 1-hour ignition at $1,000^{\circ} \mathrm{C}$. The $\mathrm{H}_{2} \mathrm{O}^{-}$and LOI contents were determined in duplicate for four separate bottles to allow between-bottle analysis of variance (anova) calculations. The constituent concentrations were obtained as the differences in weight between "as received" and dried/ignited sample weights.

Reabsorption of moisture by dried or ignited samples was measured as follows. Accurately weighed, dried/ignited samples, one from each bottle of SDO-1, were stored in separate sealed vials. The second samples from each bottle were left exposed to a humid ( $>90$ percent) laboratory atmosphere in open vials. Both groups were reweighed 8 hours later, and the reabsorbed moisture was calculated by difference. The dried (but not ignited) samples were returned to the drying oven after reabsorption, and the process was repeated through three cycles.

In addition to the standard ignition for 1 hour at $1,000^{\circ} \mathrm{C}$, three other time/temperature ignitions were done to assess variations in results attributable to drying time and temperature: 1 hour at $500^{\circ} \mathrm{C} ; 18$ hours at $500^{\circ} \mathrm{C}$, and 1 hour at $800^{\circ} \mathrm{C}$. LOI was generally determined on the "as received" sample; in some cases LOI was determined on the dried sample instead.

\section{Determination of Ferrous Iron}

For the ignited samples only, $\mathrm{FeO}$ was measured by titration with potassium dichromate to a diphenylamine sulfonate indicator endpoint using the procedure reported in Shapiro and Brannock (1962). The ash was first decom-

Table 2. Moisture: Evaluation of bottle differences

[In weight percent; d.f., degrees of freedom]

\begin{tabular}{ccl}
\hline Bottle & $\begin{array}{c}\text { Result }- \\
\text { overnight drying }\end{array}$ & \multicolumn{1}{c}{ F-test data } \\
\hline 1 & $1.57 \pm 0.023$ & d.f. $=7,13$ \\
6 & $1.64 \pm 0.015$ & $\mathrm{~F}=50.40$ \\
5 & $1.69 \pm 0.025$ & \\
4 & $1.73 \pm 0.03$ & \\
2 & $2.02 \pm 0.14$ & $\mathrm{~F}_{\text {crit }}(0.95)=2.83$ \\
7 & $2.04 \pm 0.021$ & $\mathrm{~F}_{\text {crit }}(0.99)=4.44$ \\
3 & $2.22 \pm 0.098$ & \\
Average, & $1.84 \pm 0.29$ & \\
individual & & \\
results, all & & \\
bottles & & \\
\hline Bottle & Result - & \\
& $1-\mathrm{hr}$ drying & \\
\hline 1 & 1.59 & \\
4 & 1.70 & \\
5 & 1.71 & \\
6 & 1.62 & \\
\hline
\end{tabular}

posed in the absence of air with a mixture of hydrofluoric and sulfuric acids.

\section{Measurement of CHNS}

Carbon, hydrogen, and nitrogen were measured in replicate samples from three bottles of SDO-1 using a Perkin Elmer Model 2400 CHN Elemental Analyzer; and S was measured using a Leco Model SC-132 sulfur analyzer on both "as received" and ignited samples. Measurements are based on combustion to convert the elements in sample to simple gases $\left(\mathrm{C}\right.$ to $\mathrm{CO}_{2}, \mathrm{H}$ to $\mathrm{H}_{2} \mathrm{O}, \mathrm{N}$ to $\mathrm{N}_{2}$, and $\mathrm{S}$ to $\mathrm{SO}_{2}$ ). CHN are measured by thermal conductivity after chromatographic separation, and $\mathrm{S}$ is measured by an infrared detector (Leco, 1980, Perkin Elmer, 1988). These data allow estimation of homogeneity with respect to individual $\mathrm{C}, \mathrm{H}, \mathrm{N}$, and $\mathrm{S}$ samples, which had not been possible before.

\section{RESULTS AND DISCUSSION}

\section{Moisture $\left(\mathrm{H}_{2} \mathrm{O}^{-}\right)$}

We have noted that several laboratories included moisture in their LOI values, since they ignited samples "as received." Subtle changes in the degree to which samples exhibit hygroscopic behavior and absorb atmospheric moisture are perhaps related to the between-bottle variation in the ratio of clay minerals to other principal components, namely pyrite and organic matter. Such between-bottle variation is evident from the inhomogeneity found with respect to $\mathrm{SiO}_{2}, \mathrm{Al}_{2} \mathrm{O}_{3}$, and total iron reported as $\mathrm{Fe}_{2} \mathrm{O}_{3}$, which has been documented (Kane and others, 1990).

$\mathrm{H}_{2} \mathrm{O}^{-}$measured in this work varied significantly between bottles, as shown in table 2 . Overnight drying was used in this study; some laboratories (for example, Frost and others, 1985) employed much briefer drying times of 1 hour. Presumably, this short time was intended to avoid the potential loss of volatile organic compounds from the sample (Jackson and others, 1987). Our results are among the highest moisture contents of the data contributed to the SDO-1 characterization, suggesting a high bias due to loss of organic matter during drying. However, a considerable fraction of the lost weight was restored as reabsorbed moisture in rather short (8-hour) intervals, and more than 85 percent of the lost weight was restored in a 3-day exposure to moist air (fig. 1). Also, moisture determined subsequently in our laboratory using a drying time of 1 hour gave the same moisture results as 18-hour drying had given. Differences in moisture content from bottle to bottle seem to be the principal source of between-laboratory variance in the moisture determination and, therefore, also a principal factor in the reported between-laboratory variance for LOI. 


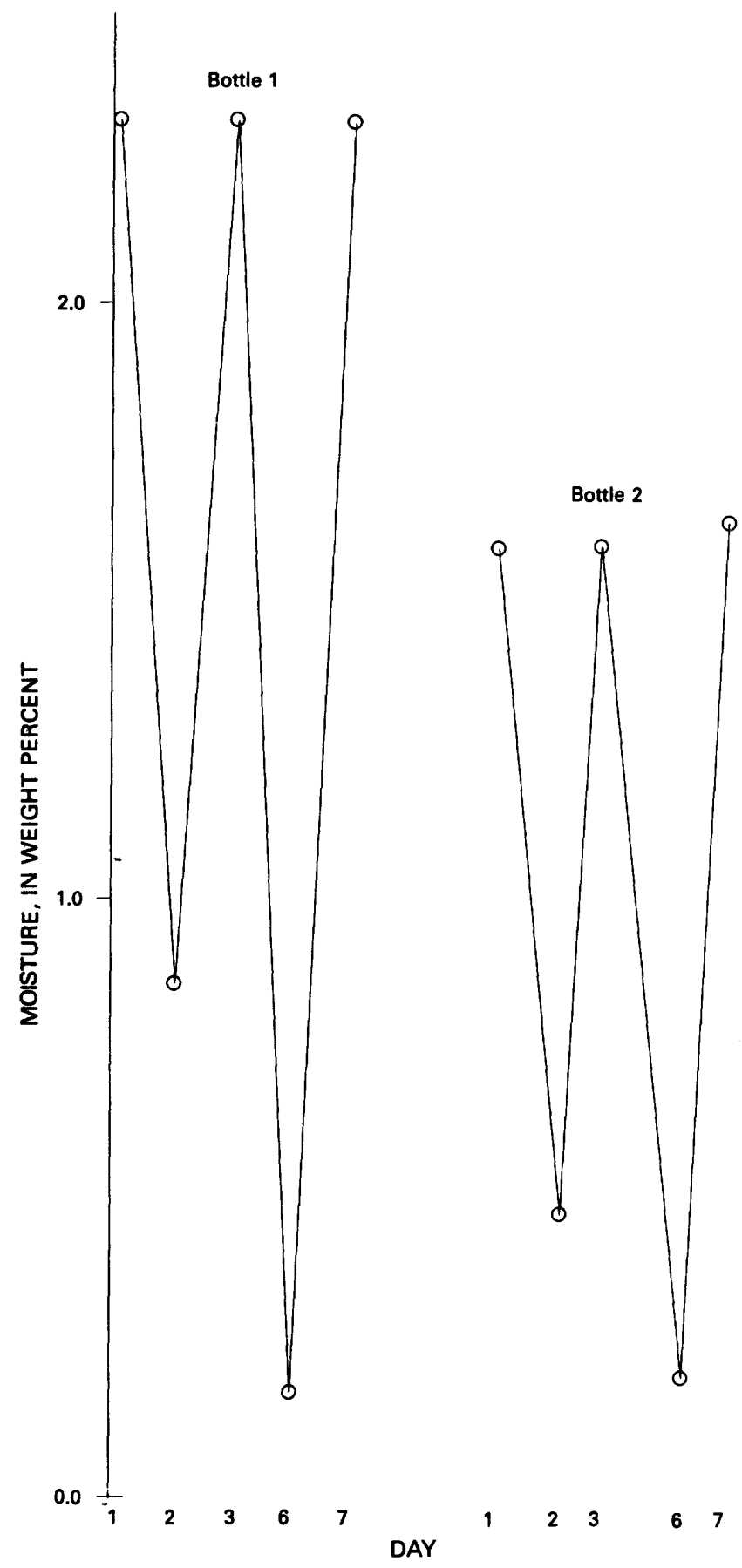

Figure 1. Moisture results after cyclic drying and reabsorption. Days 1, 3, 7: after overnight drying; day 2: after 8 hours exposure to moist air; and day 6: after 3 days exposure to moist air.

\section{LOI}

In this study, as well as in the original compiled data, LOI measured both on an "as received" basis and on a dry basis showed considerable variation between bottles (table 3). This variation suggests that organic matter, as well as clay, pyrite, and moisture, are heterogeneously distributed between bottles. The compiled total carbon, organic carbon, and $\mathrm{CO}_{2}$ results in table 1 do not allow adequate homogeneity evaluations, as reported. Bottle-to-bottle moisture variation, discussed above, accounts for approximately half the observed LOI between-bottle variance; between-bottle variation of organic matter could account for most of the remaining variance. CHN data from this study appear to confirm this theory.

Weight gain from pyritic and ferrous iron oxidation and possibly from sulfur fixation also accounts for some of the observed between-bottle variance in LOI. These aspects of between-bottle and between-laboratory variance in LOI will be discussed in the next two sections.

The reactions that potentially occur during ignition are summarized in table 4. All are well-documented through differential thermal analysis (DTA) studies (Liptay, 1971; Warne, 1979) to be dependent on ignition temperature. These studies indicate that $\mathrm{CO}_{2}$ will not be lost from calcite below $800^{\circ} \mathrm{C}$; however, magnesite and siderite lose $\mathrm{CO}_{2}$ at 580 to $690^{\circ} \mathrm{C}$ and 540 to $620^{\circ} \mathrm{C}$, respectively. Similarly, pyrite oxidizes to hematite with release of $\mathrm{SO}_{3}$ in the neighborhood of $580^{\circ} \mathrm{C}$. Because DTA studies are carried out under very different ignition conditions from those for determination of LOI, the reaction temperatures found through DTA experiments do not duplicate those for ignition reactions; however, DTA results do indicate potential sources of variation in LOI with ignition temperature. These factors collectively could explain the observed between-laboratory variation that cannot be attributed to between-bottle moisture and (or) organic-matter variations.

One-way anova with temperature as the classification variable (table 3) shows the significance of temperature variation. Two-way anova shows the interaction between temperature and bottle as a source of variation. That interaction is reflected in the nonparallel plots of LOI vs ignition temperature for the several bottles included in this study (fig. 2). This interaction implies that each of the contributions of each of the reactions in table 4 to the measured LOI varies somewhat bottle to bottle.

\section{FeO and S in Ignited Sample}

Total iron reported as $\mathrm{Fe}_{2} \mathrm{O}_{3}$ is $9.36 \pm 0.22$ percent in SDO-1; however, based on geochemical and petrological evidence (Leventhal and Hosterman, 1982, Frost and others, 1985), little or none of the iron present occurs as ferric iron. All iron is instead thought to occur either in pyrite or as ferrous iron, occurring mainly in clays, oxides, and to a lesser extent in carbonates. Experimentally, both $\mathrm{FeO}$ and $\mathrm{S}$ were determined in samples after ignition. Results of those measurements are given in table 5 .

The data show essentially complete oxidation of ferrous oxide/carbonate and pyrite at all three ignition 
Table 3. Loss on ignition (in weight percent): Evaluation of bottle and ignitiontemperature differences

[LOI, loss on ignition; d.f., degrees of freedom; leaders (-), no data]

\begin{tabular}{|c|c|c|c|c|}
\hline \multirow[b]{2}{*}{ Bottle } & \multicolumn{4}{|c|}{ Results for $L O I$ as received } \\
\hline & $550^{\circ} \mathrm{C}, 1 \mathrm{hr}$ & $550^{\circ} \mathrm{C}, 18 \mathrm{hr}$ & $800^{\circ} \mathrm{C}, 1 \mathrm{hr}$ & $1,000^{\circ} \mathrm{C}, 1 \mathrm{hr}$ \\
\hline 1 & 19.49 & 19.43 & 20.89 & 22.69 \\
\hline 2 & 19.97 & - & 21.88 & 23.22 \\
\hline 3 & 19.95 & - & 22.19 & 23.73 \\
\hline 4 & 19.40 & 20.01 & 21.04 & 22.61 \\
\hline 5 & - & - & - & 23.72 \\
\hline 6 & - & - & - & 21.58 \\
\hline 7 & 19.49 & - & - & 23.05 \\
\hline 8 & 18.65 & 18.85 & 20.15 & 21.58 \\
\hline Average & $19.25 \pm 0.54$ & $19.43 \pm 0.53$ & $20.82 \pm 0.86$ & $23.03 \pm 0.59$ \\
\hline \multicolumn{5}{|l|}{ F-test data: } \\
\hline $\begin{array}{l}\text { d.f. } \\
\text { F }\end{array}$ & $\begin{array}{r}2,6 \\
13.3\end{array}$ & & $\begin{array}{r}3,4 \\
115.6\end{array}$ & $\begin{array}{c}4,9 \\
80.44\end{array}$ \\
\hline $\begin{array}{l}\text { Probability LOI } \\
\text { same, all bottles }\end{array}$ & 0.007 & & 0.001 & $<0.001$ \\
\hline $\mathrm{F}_{\text {crit }}(0.95)$ & 5.14 & & & 6.59 \\
\hline
\end{tabular}

Table 4. Reactions during ignition of sample

\begin{tabular}{llc}
\hline \multicolumn{1}{c}{ Reaction } & \multicolumn{1}{c}{$\begin{array}{c}\text { Reaction temperatures for specific } \\
\text { minerals (Liptay, 1971; Warne, 1979) }\end{array}$} \\
\hline $\mathrm{MCO}_{3} \rightarrow \mathrm{MO}+\mathrm{CO}_{2} \uparrow$ & Siderite: & $540-620^{\circ} \mathrm{C}$ \\
& Magnesite: & $580-690^{\circ} \mathrm{C}$ \\
$\mathrm{FeS}_{2} \rightarrow \mathrm{Fe}_{2} \mathrm{O}_{3}+\mathrm{SO}_{2} \uparrow$ & Calcite: & $>800^{\circ} \mathrm{C}$ \\
& Begins at: & $580^{\circ} \mathrm{C}$ \\
$\mathrm{Hydrous}$ clays $\rightarrow$ anhydrous $+\mathrm{H}_{2} \mathrm{O} \uparrow$ & Complete reaction: & $>1,000^{\circ} \mathrm{C}$ \\
& Illite: & $500-550^{\circ} \mathrm{C}$ \\
& Kaolinite: & $550-620^{\circ} \mathrm{C}$ \\
Organic matter $\rightarrow \mathrm{CO}_{2}+\mathrm{H}_{2} \mathrm{O}$ & Montmorillonite: & $700-730^{\circ} \mathrm{C}$ \\
& Essentially complete & $\geq 1500^{\circ} \mathrm{C}$ \\
\hline
\end{tabular}

temperatures $\left(500^{\circ} \mathrm{C}, 800^{\circ} \mathrm{C}\right.$, and $\left.1,000^{\circ} \mathrm{C}\right)$. This result contrasts with the Lechler and Desilets (1987) report that oxidation is complete only at $100^{\circ} \mathrm{C}$ ignition. The weight gain of sample during ignition due to iron oxidation, therefore, should be the same regardless of ignition temperature. Assuming that all iron in SDO-1 is ferrous initially, and that it is totally oxidized to hematite $\left(\mathrm{Fe}_{2} \mathrm{O}_{3}\right)$, that gain amounts to 2.25 percent, as shown in table 5 .

Our data also indicate extensive sulfur retention $(>20$ percent of initial S) at $500^{\circ} \mathrm{C}$ ignition, which decreases to approximately 10 -percent retention at $1,000^{\circ} \mathrm{C}$ ignition. Based on the data in table 5 , the weight gain of sample will be maximum, approximately 2 percent, for ignition at $500^{\circ} \mathrm{C}, 1.5$ percent for ignition at $800^{\circ} \mathrm{C}$, and 0.75 percent for ignition at $1,000^{\circ} \mathrm{C}$. The calculation is given in table 5 .

\section{CHN in Both "As Received" and Ignited Sample}

Comparison of data for $\mathrm{C}$ and $\mathrm{H}$ in "as received" and ignited sample indicates both complete oxidation of all organic matter and nearly complete removal of constitutional water at $500^{\circ} \mathrm{C}$. Bottle differences exist for $\mathrm{C}$ at both the 95-percent and 99-percent confidence levels but are less conclusive for $\mathrm{H}$, existing only at the 99-percent confidence level (table 6). The data also appear to indicate that carbonate remains in $500^{\circ} \mathrm{C}$ ash but is totally expelled as $\mathrm{CO}_{2}$ in $800^{\circ} \mathrm{C}$ ash.

\section{ANALYTICAL TOTAL}

LOI as measured was shown above to be low by 2.71 percent $\left(1,000^{\circ} \mathrm{C}\right)$ to 4.47 percent $\left(500^{\circ} \mathrm{C}\right)$, based on weight 
Table 5. FeO and $\mathrm{S}$ in ignited sample residue and weight gain calculations (in weight percent) [d.f., degrees of freedom; leaders (-), no data]

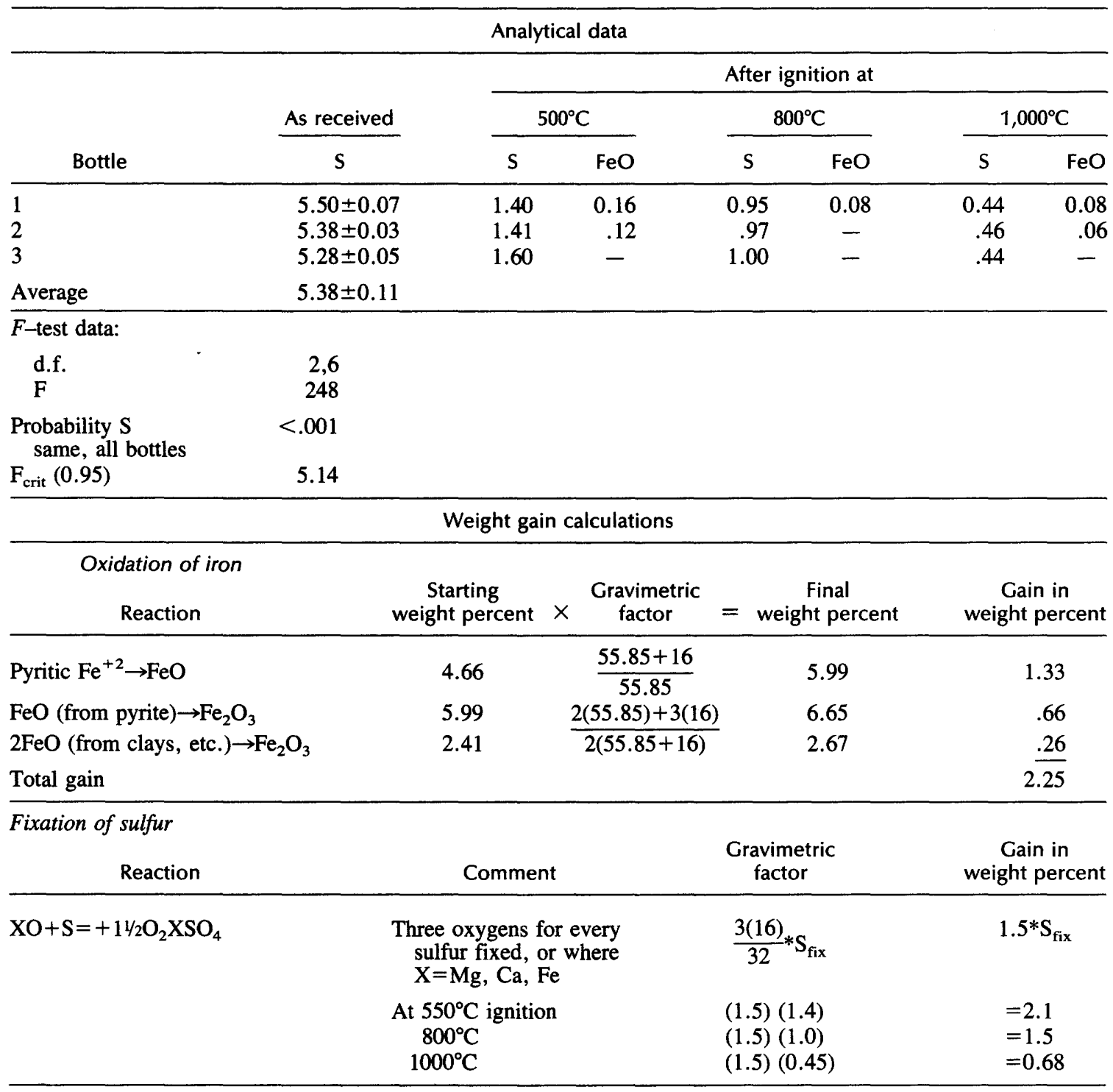

Table 6. $\mathrm{CHN}$ results for SDO-1

[In weight percent; d.f., degree of freedom]

\begin{tabular}{|c|c|c|c|c|c|c|c|c|c|}
\hline \multirow[b]{3}{*}{ Bottle } & \multirow{2}{*}{\multicolumn{3}{|c|}{ As received }} & \multicolumn{6}{|c|}{ After ignition } \\
\hline & & & & \multicolumn{3}{|c|}{ at $500^{\circ} \mathrm{C}$} & \multicolumn{3}{|c|}{ at $800^{\circ} \mathrm{C}$} \\
\hline & C & $\mathrm{H}$ & $\mathrm{N}$ & C & $\mathrm{H}$ & $N$ & C & $\mathrm{H}$ & $N$ \\
\hline 1 & 9.92 & 1.23 & 0.31 & 0.22 & $0.04 \mathrm{C}$ & 0.06 & $<0.01$ & $<0.01$ & 0.05 \\
\hline 2 & 9.66 & 1.38 & .33 & .17 & .04 & .04 & $<.01$ & $<.01$ & .06 \\
\hline 3 & $\underline{9.59}$ & 1.41 & .35 & .18 & .05 & .07 & $<.01$ & $<.01$ & .06 \\
\hline Average & 9.72 & 1.34 & .33 & .187 & .043 & .057 & $<.01$ & $<.01$ & .057 \\
\hline $\mathrm{CO}_{2}$ equivalent & & & & & $63-0.8$ & & & $<0.03$ & \\
\hline \multicolumn{10}{|l|}{ F-test data: } \\
\hline $\begin{array}{l}\text { d.f. } \\
\text { F }\end{array}$ & $\begin{array}{r}2,6 \\
248\end{array}$ & 5.92 & 2.66 & & & & & & \\
\hline Probability bottles same & $<.001$ & 1.038 & 8.15 & & & & & & \\
\hline
\end{tabular}




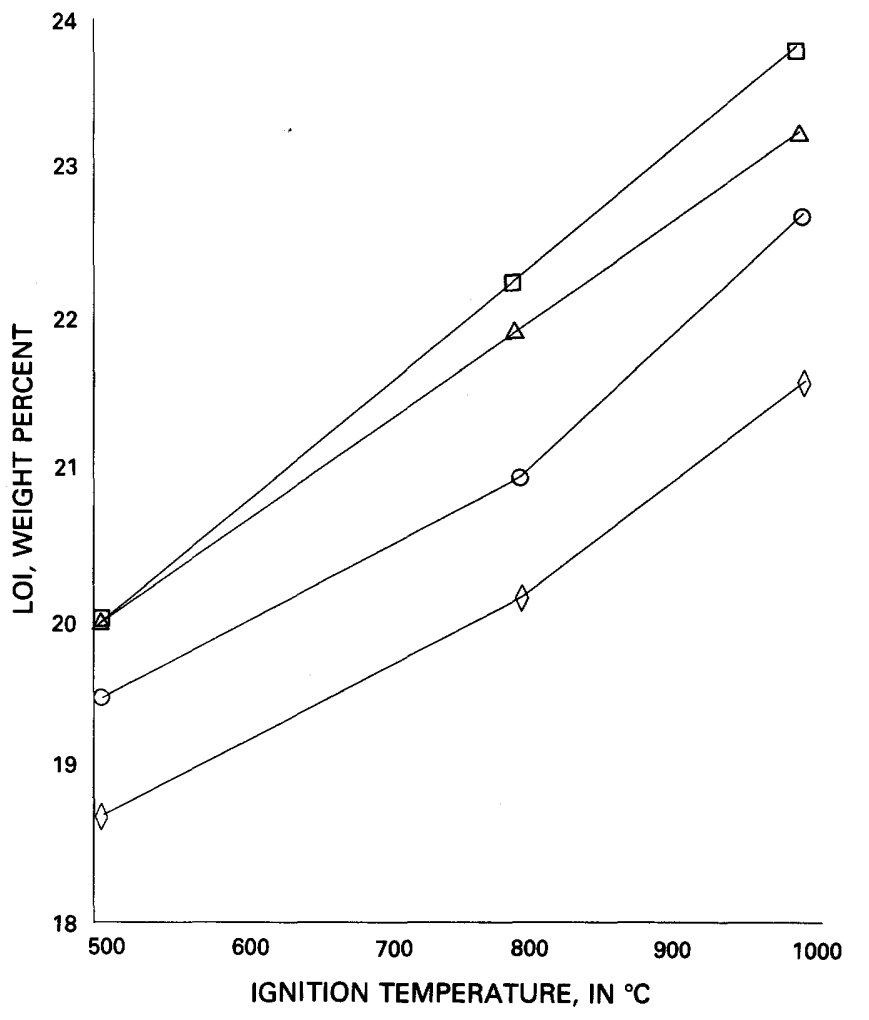

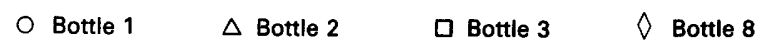

Figure 2. LOI as a function of temperature, illustrating interaction between temperature and bottle components of variance.

gains during ignition due to iron oxidation and sulfur fixation that varied as a function of ignition temperature and on failure to expel $\mathrm{CO}_{2}$ at $500^{\circ} \mathrm{C}$. The sum of volatile components, based on correction of measured LOI, should therefore be $23.92-25.77$ percent. The original analytical summation of recommended concentrations for (nonvolatile) major rock-forming oxides was calculated having iron appropriately included as $\mathrm{Fe}^{+2}$ from pyrite or $\mathrm{FeO}$. That summation is 75.81 percent without the volatile components, and 99.73 percent to 101.58 percent including volatile components, based on LOI corrections for $500^{\circ} \mathrm{C}$ and $1,000^{\circ} \mathrm{C}$ ignition (table 7). Alternatively, the sum of volatile components can be estimated to be 23.16 percent, as shown in table 7, leading to a final summation of 98.97 percent.

\section{CONCLUSION}

With proper understanding of the components in measured LOI, accurate totals of $100 \pm 2$ percent can be achieved for sulfur- and (or) organic-rich samples, of which SDO-1 is representative. The acceptability of the
Table 7. Alternative estimates of the sum of volatile components

[Leaders (-), no data]

\begin{tabular}{|c|c|c|c|}
\hline \multicolumn{4}{|c|}{ Volatile components as corrected } \\
\hline & $500^{\circ} \mathrm{C}$ & $800^{\circ} \mathrm{C}$ & $1,000^{\circ} \mathrm{C}$ \\
\hline LOI & 19.25 & 20.83 & 23.06 \\
\hline $\mathrm{SO}_{3}$ in ash & 1.40 & 1.0 & .45 \\
\hline Gain from $\mathrm{Fe}$ oxidation & 2.26 & 2.26 & 2.26 \\
\hline $\mathrm{CO}_{2}$ residue & 1.01 & - & - \\
\hline Total volatile components & 23.92 & 24.09 & 25.77 \\
\hline Total oxides* & 75.81 & $\underline{75.81}$ & 75.81 \\
\hline Analytical summation & 99.73 & 99.90 & 101.58 \\
\hline \multicolumn{4}{|c|}{$\begin{array}{l}\text { Estimate of individual volatiles } \\
\text { from compilation averages }\end{array}$} \\
\hline Organic $=\mathrm{C}_{\mathrm{org}} \times 1.3$ & 12.57 & & \\
\hline $\mathrm{CO}_{2}$ & 1.01 & & \\
\hline $\mathrm{H}_{2} \mathrm{O}^{+}$ & 2.63 & & \\
\hline $\mathrm{H}_{2} \mathrm{O}^{-}$ & 1.60 & & \\
\hline$S$ & 5.35 & & \\
\hline Total volatile compoments & 23.16 & & \\
\hline Total oxides* & 75.81 & & \\
\hline Analytical summation & 98.97 & & \\
\hline
\end{tabular}

*Summation includes pyritic iron as $\mathrm{Fe}^{+2}$, balance of total iron as $\mathrm{FeO}$, in weight percent.

totals does not ensure that the individual recommended concentrations are correct but nonetheless adds to our confidence in those concentrations.

\section{REFERENCES}

Abbey, Sidney, 1983, Studies in "Standard Samples" of silicate rocks and minerals, 1969-1982: Geological Survey of Canada Paper 83-15, $114 \mathrm{p}$.

Frost, J.K., Zierath, D.L., and Shimp, N.F., 1985, Chemical Composition and Geochemistry of the new Albany Shale Group (Devonian-Mississippian) in Illinois: Illinois State Geological Survey Contract/Grant Report 1985-4, 134 p.

Gladney, E.S., and Roelandts, Iwan, 1988, 1987 compilation of elemental concentration data for USGS BHVO-1, MAG-1, QLO-1, RGM-1, SCo-1, SDC-1, SGR-1, and STM-1: Geostandards Newsletter, v. 12, p. 253-362.

Jackson, L.L., Brown, F.W., and Neil, S.T., 1987, Major and minor elements requiring individual determination, classical whole rock analysis, and rapid rock analysis, in Baedecker, P.A., ed., Methods for geochemical analysis: U.S. Geological Survey Bulletin 1770, p. G1-G23.

Kane, J.S., Arbogast, B.F., and Leventhal, J.S., 1990, Characterization of Devonian Ohio Shale SDO-1 as a USGS 
geochemical reference sample: Geostandards Newsletter, v. 14, p. 169-196.

Lechler, P.J., and Desilets, M.O., 1987, A review of the use of loss on ignition as a measure of total volatiles in whole rock analysis: Chemical Geology, v. 63, p. 341-344.

Leco Corporation, 1980. Leco SC-132 sulfur system instruction manual 200-192.

Leventhal, J.S., and Hosterman, J.W., 1982, Chemical and mineralogical analysis of Devonian Black Shale samples from Martin County, Kentucky; Carroll and Washington Counties, Ohio; Wise County, Virginia; and Overton County, Tennessee, USA: Chemical Geology, v. 37, p. 239-264.
Liptay, G., ed., 1971, Atlas of thermoanalytical plots: Amsterdam, Hayden and Sons, v. 1-3, p. 116, 161, 162, see especially v. 1 , curve 21 .

Perkin Elmer Corporation, 1988. 2400 CHN elemental analyzer instruction manual 0993-7147.

Potts, P.J., 1987, A handbook of silicate rock analysis: Blackie, Glasgow.

Shapiro, Leonard, and Brannock, W.W., 1962, Rapid analysis of silicate, carbonate, and phosphate rocks: U.S. Geological Survey Bulletin 1144A, 56 p.

Warne, S. St. J., 1979, Differential thermal analysis of coal minerals in Analytical methods for coal and coal products, v. 3, Academic Press, p. 447-447. 

Chapter G

Analysis of SDO-1 Pressed Powder Pellets
by X-ray Fluorescence-A Note of Caution

By OTTO C. KOPP and FRANCIS C. FURMAN

U.S. GEOLOGICAL SURVEY BULLETIN 2046

THE USGS REFERENCE SAMPLE DEVONIAN OHIO SHALE SDO-1 



\title{
Analysis of SDO-1 Pressed Powder Pellets by X-ray Fluorescence-A Note of Caution
}

\author{
By Otto C. Kopp and Francis C. Furman ${ }^{1}$
}

SDO-1 is an organic-rich shale relatively high in sulfur. Caution should be used in analyzing this shale by $\mathrm{X}$-ray fluorescence (XRF) using a pressed-pellet technique, such as described by Wheeler (1984). Most available standards, used to generate the calibration curves and to establish corrections for any matrix effects encountered in the analysis, contain lower concentrations of sulfur and organic matter than SDO-1 and, therefore, have matrix characteristics quite different from SDO-1.

We attempted to analyze SDO-1 using our standard procedures. Representative splits were air-dried at $105^{\circ} \mathrm{C}$ for 1 hour and ground in a swing mill ("shatterbox") for 6 minutes; the pellets were pressed at 30,000 psi for 1 minute. The concentrations for most elements did not agree with the recommended values for SDO-1 (Kane and others, 1990). After considering several sources of error, we concluded that our results were rendered invalid primarily by the high concentrations of organic matter and sulfur in SDO-1. Our analytical protocol is based on standard reference materials that are low in organic matter and sulfur. The maximum organic carbon concentration in our standards is unknown while the maximum sulfur concentraztion is 1.89 percent. These concentrations are 9.6 percent $C_{\text {org }}$ and 5.35 percent $\mathrm{S}$ in SDO-1.

Perhaps we had become complacent because our analytical protocol for clays, shales, and soils had performed so well over a period of several years. Only when the interelement correction steps were reexamined did we realize the magnitude of the errors that could be introduced. Although the use of elements such as sulfur to make interelement corrections for major elements may seem unusual, the presence of sulfur reflects matrix effects that

Manuscript approved for publication September 15, 1992.

${ }^{1}$ University of Tennessee can enhance (or absorb) some of the secondary X-rays generated for other elements.

Hence, a word of caution is advisable to all who use pressed pellets to perform XRF analyses of atypical samples such as SDO-1. Results are questionable if the net intensities or calculated concentrations for any elements, and especially those elements used in the matrix correction steps, fall outside the ranges of the standards used in generating the protocol. Information about the reference standards and interelement correction steps, possibly unknown to most users, can be critical to analytical accuracy.

Matrix effects can be reduced by analyzing samples fused with a flux such as lithium tetraborate (for example, Spies and others, chap. B, this vol.). However, the fusion technique is more time-consuming and costly than preparing pellets; and the addition of a flux reduces the concentrations of trace elements that might be determined simultaneously, making their determination less precise or even diluting them to concentrations below their detection limits.

The problem will ultimately be resolved when new standards with high sulfur concentrations and organic-rich matrices (such as SDO-1) are added to the analytical protocol.

\section{REFERENCES}

Kane, J.S., Arbogast, Belinda, and Leventhal, Joel, 1990, Characterization of Devonian Ohio Shale SDO-1 as a USGS Geochemical reference sample, Geostandards Newsletter, 14:169-196.

Wheeler, B.D., 1984, Analysis of rocks and minerals using energy dispersive X-ray fluorescence: XRF Note XRF-4, EG\&G ORTEC, Oak Ridge, Tenn., and Abstract, 1984 GEOEXPO: Beijing, China, August 1-10, 1984.50. 






\section{SELECTED SERIES OF U.S. GEOLOGICAL SURVEY PUBLICATIONS}

\section{Periodicals}

Earthquakes \& Volcanoes (issued bimonthly).

Preliminary Determination of Epicenters (issued monthly).

\section{Technical Books and Reports}

Professional Papers are mainly comprehensive scientific reports of wide and lasting interest and importance to professional scientists and engineers. Included are reports on the results of resource studies and of topographic, hydrologic, and geologic investigations. They also include collections of related papers addressing different aspects of a single scientific topic.

Bulletins contain significant data and interpretations that are of lasting scientific interest but are generally more limited in scope or geographic coverage than Professional Papers. They include the results of resource studies and of geologic and topographic investigations, as well as collections of short papers related to a specific topic.

Water-Supply Papers are comprehensive reports that present significant interpretive results of hydrologic investigations of wide interest to professional geologists, hydrologists, and engineers. The series covers investigations in all phases of hydrology, including hydrogeology, availability of water, quality of water, and use of water.

Circulars present administrative information or important scientific information of wide popular interest in a format designed for distribution at no cost to the public. Information is usually of short-term interest.

Water-Resources Investigations Reports are papers of an interpretive nature made available to the public outside the formal USGS publications series. Copies are reproduced on request unlike formal USGS publications, and they are also available for public inspection at depositories indicated in USGS catalogs.

Open-File Reports include unpublished manuscript reports, maps, and other material that are made available for public consultation at depositories. They are a nonpermanent form of publication that may be cited in other publications as sources of information.

\section{Maps}

Geologic Quadrangle Maps are multicolor geologic maps on topographic bases in 7.5- or 15-minute quadrangle formats (scales mainly $1: 24,000$ or $1: 62,500$ ) showing bedrock, surficial, or engineering geology. Maps generally include brief texts; some maps include structure and columnar sections only.

Geophysical Investigations Maps are on topographic or planimetric bases at various scales; they show results of surveys using geophysical techniques, such as gravity, magnetic, seismic, or radioactivity, which reflect subsurface structures that are of economic or geologic significance. Many maps include correlations with the geology.

Miscellaneous Investigations Series Maps are on planimetric or topographic bases of regular and irregular areas at various scales; they present a wide variety of format and subject matter. The series also includes 7.5-minute quadrangle photogeologic maps on planimetric bases that show geology as interpreted from aerial photographs. Series also includes maps of Mars and the Moon.
Coal Investigations Maps are geologic maps on topographic or planimetric bases at various scales showing bedrock or surficial geology, stratigraphy, and structural relations in certain coal-resource areas.

Oil and Gas Investigations Charts show stratigraphic information for certain oil and gas fields and other areas havirg petroleum potential.

Miscellaneous Field Studies Maps are multicolor or black-antwhite maps on topographic or planimetric bases for quadrangle or irregular areas at various scales. Pre-1971 maps show bedrock geology in relation to specific mining or mineral-deposit problems; post-1971 maps are primarily black-and-white maps on various subjects such as environmental studies or wilderness mineral investigations.

Hydrologic Investigations Atlases are multicolored or blackand-white maps on topographic or planimetric bases presenting a wide range of geohydrologic data of both regular and irregular aress; principal scale is $1: 24,000$, and regional studies are at $1: 250,000$ scale or smaller.

\section{Catalogs}

Permanent catalogs, as well as some others, giving comprehensive listings of U.S. Geological Survey publications are availatle under the conditions indicated below from the U.S. Geological Survey, Map Distribution, Box 25286, Bldg. 810, Federal Center, Denver, CO 80225. (See latest Price and Availability List.)

"Publications of the Geological Survey, 1879-1961" may be purchased by mail and over the counter in paperback book form and as a set of microfiche.

"Publications of the Geological Survey, 1962-1970" may be purchased by mail and over the counter in paperback book form and as a set of microfiche.

"Publications of the U.S. Geological Survey, 1971-1981" may be purchased by mail and over the counter in paperback book form (two volumes, publications listing and index) and as a set of microfiche.

Supplements for 1982, 1983, 1984, 1985, 1986, and for subsequent years since the last permanent catalog may be purchased by mail and over the counter in paperback book form.

State catalogs, "List of U.S. Geological Survey Geologic and Water-Supply Reports and Maps For (State)," may be purchased by mail and over the counter in paperback booklet form only.

"Price and Availability List of U.S. Geological Survey Publications," issued annually, is available free of charge in paperba $\mathrm{k}$ booklet form only.

Selected copies of a monthly catalog "New Publications of the U.S. Geological Survey" are available free of charge by mail or may be obtained over the counter in paperback booklet form only. Thcse wishing a free subscription to the monthly catalog "New Publications of the U.S. Geological Survey" should write to the U.S. Geological Survey, 582 National Center, Reston, VA 22092.

Note.-Prices of Government publications listed in older catalogs, announcements, and publications may be incorre t. Therefore, the prices charged may differ from the prices in catalogs, announcements, and publications. 


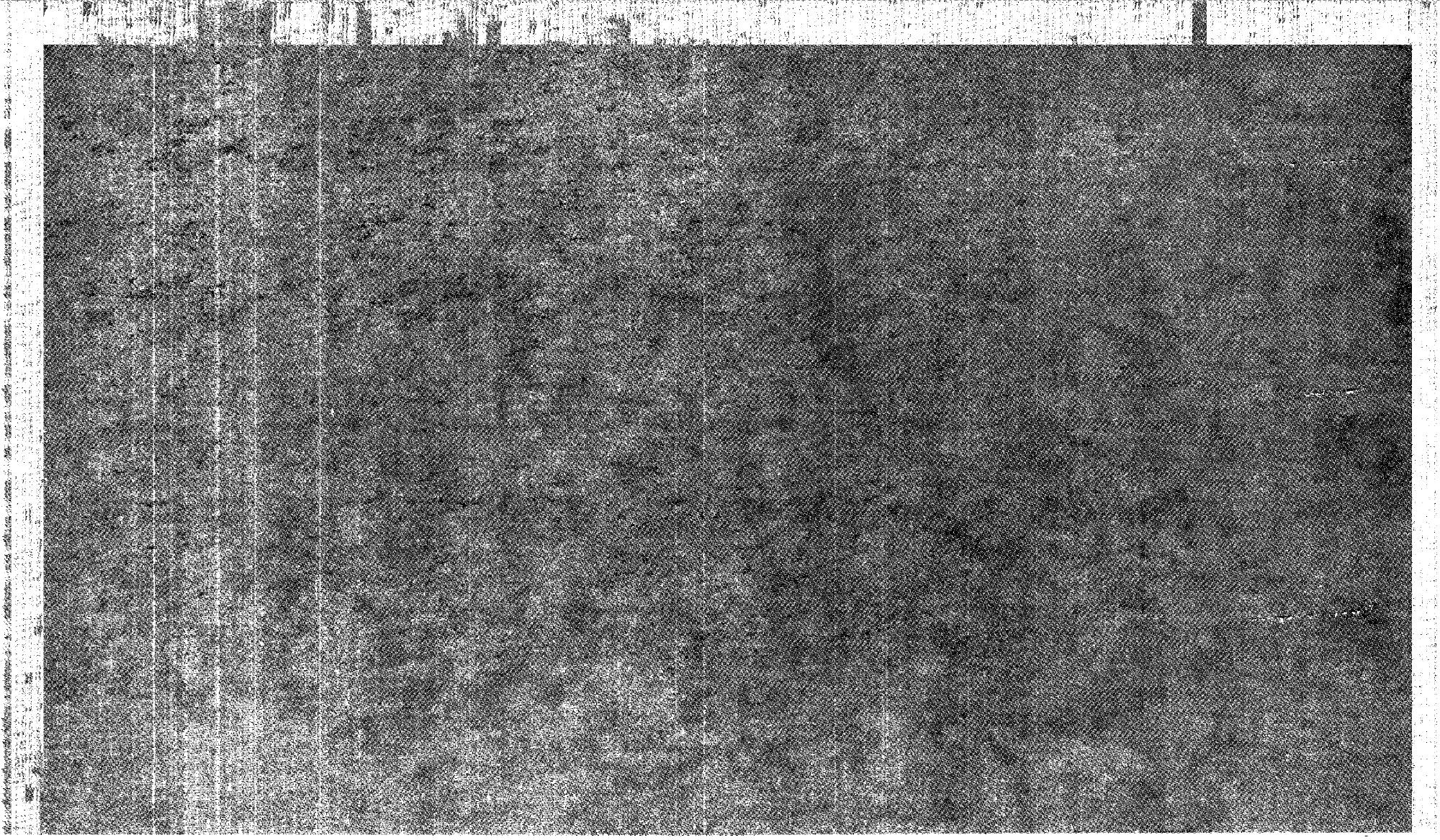

